\title{
Descriptions of Reference \\ LWR Facilities for Analysis of Nuclear Fuel Cycles Appendixes
}
K. J. Schneider
T. J. Kabele

September 1979

Prepared for the U.S. Department of Energy under Contract EY-76-C-06-1830

Pacific Northwest Laboratory Operated for the U.S. Department of Energy by Battelle Memorial Institute 
This report was prepared as an account of work sponsored by the United States Covernment. Neither' the United States nor the Department of Energy, nor any of their employees, nor any of their contractors, subcontractors, or their employees, makes any warranty, express or implied, or assumes any legal liability or responsibility for the accuracy, completeness or usefulness of any information, apparatus, product or process disclosed, or represents that its use would not infringe privately owned rights.

The views, opinions and conclusions contained in this report are those of the contractor and do not necessarily represent those of the United States Covernment or the United States Department of Energy.

\author{
PACIFIC NORTHWEST LABORATORY \\ operated by \\ BATTELLE \\ for the \\ UNITED STATES DEPARTMENT OF ENERGY \\ Under Contract EY-76-C-06-1830
}
Printed in the United States of America
Available from
National Technical Information Service
United States Department of Cornmerce
5285 Port Royal Road
Springfield, Virginia 22151

Price: Printed Copy s

$\therefore$ Microfiche $\$ 3.00$

NTIS
*Pages Seiling Price

$\begin{array}{lll}001-025 & 54.00 \\ 026-050 & 54.50 \\ 051-075 & 55.25 \\ 076-100 & 56.00 \\ 101-125 & 56.50 \\ 126-150 & 57.25 \\ 151-175 & \$ 8.00 \\ 176-200 & 59.00 \\ 201-225 & 59.25 \\ 226-250 & 59.50 \\ 251-275 & 510.75 \\ 266-300 & 511.00\end{array}$


DESCRIPTIONS OF REFERENCE LWR FACILITIES

FOR ANALYSIS OF NUCLEAR FUIEL CYCLES

APPENDIXES

K. J. Schneider, Study Coordinator

T. J. Kabele, Project Manager

September 1979

Prepared for

the U.S. Department of Energy

under Contract EY-76-C-06-1830

Pacific Northwest Laboratory

Richland, Washington 99352 
• 


\title{
DESCRIPTIONS OF REFERENCE LWR FACILITIES FOR \\ ANALYSIS OF NUCLEAR FUEL CYCLES
}

\section{APPENDIXES}

The appendixes present the calculations that were used to derive the release factors discussed for each fuel cycle facility in Volume I. Appendix $A$ presents release factor calculations for a surface mine, underground mine, milling facility, conversion facility, diffusion enrichment facility, fuel fabrication facility, PWR, BWR and reprocessing facility. Appendix B contains additional release factors calculated for a BWR, PWR and a reprocessing facility. Appendix $\mathrm{C}$ presents release factors for a $\mathrm{UO}_{2}$ fuel fabrication facility.

\section{CONTRIBUTORS}

\author{
Appendix A - T. B. Powers \\ Appendix B - M. H. Arndt \\ J. K. Young \\ Appendix C - R. L. McKeever \\ W. A. Muckerheide
}





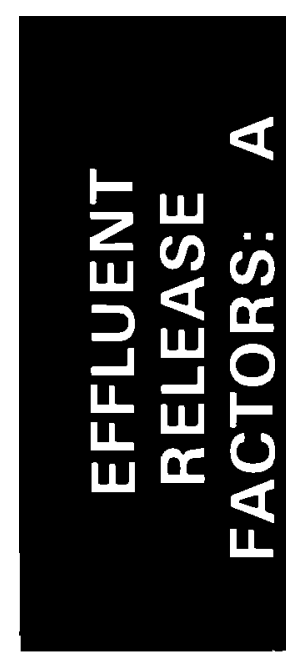


APPENDIX A

\section{DERIVATIONS OF EFFLUENT RELEASE FACTORS FOR}

THE REFERENCE FUEL CYCLE FACILITIES

T. B. Powers

Appendix A consists of ten sections, each pertaining to a separate facility of the nuclear fuel cycle. Two summary tables at the beginning of each section tabulate results of subsequent release factor calculations, as well as index the specific release factor calculations. One of the summary tables deals with chemical release factors and the other deals with radionuclide release factors.

Both tables include a column indicating the reliability of the release factor given. A reliability rating of 1,2, or 3 was assigned based on the following assumptions:

1. A reasonable but unsubstantiated guess

2. Calculated or based on laboratory measurement

3. Based on some plant experience

The release factor calculations follow the summary tables. Release factors for mining, milling, purification and conversion, and fuel fabrication are the result of kilograms effluent per day as the numerator and kilograms of contained uranium input per day to the facility as the denominator; for diffusion enrichment, kilograms per day or product are used as the denominator. Chemical release factors for BINR, PWR and reprocessing are the result of kilograms effluent per day as the numerator and kilograms of contained uranium charged to the reactor as the denominator. Radioactive release factors for BUR. PWR or reprocessing use the total kilograms of the radioactive element or radioisotope processed each day for the denominator. This is done because the ENFORM code has the capability of following changes in isotopic content with time. 
As an example of how to use Appendix A, suppose you want to find the release factor for rock dust as an airborne effluent in the surface mine.

- Find the surface mine section and look in the chemical release factor summary table to find the heading "rock dust."

- Under the airborne column you find the release factor is given $\left(7 \times 10^{-1}\right)$ as well as a reliability factor (1) and a reference calculation index number $(1-6)$.

- If you want to see how the release factor was actually calculated, you find the reference calculation index number for "rock dust" (1-6) on the following pages of calculations.

Reference numbers given in the release factor calculations can be found in the reference list at the end of Appendix $A$. 


\section{CONTENTS}

SURFACE MINE CHEMICAL RELEASE FACTORS . . . . . . . . . A.5 SURFACE MINE RADIONUCLIDE RELEASE FACTORS . . . . . . . A.7 REFERENCE CALCULATIONS: SURFACE MINE . . . . . . . . . A.8 UNDERGROUND MINE CHEMICAL RELEASE FACTORS . . . . . . . A.13 UNDERGROUND MINE RADIONUCLIDE RELEASE FACTORS. . . . . . A.15 REFERENCE CALCULATIONS: UNDERGROUND MINE . . . . . . A. A 16 ACID LEACH MILL CHEMICAL RELEASE FACTORS . . . . . A.22 ACID LEACH MILL RADIONUCLIDE RELEASE FACTORS . . . . . A.24 REFERENCE CALCULATIONS: MILLING FACILITY . • • • . . A. A 25 CONVERSION FACILITY CHEMICAL RELEASE FACTORS . . . . . . A.35 CONVERSION FACILITY RADIONUCLIDE RELEASE FACTORS • . . . . A.36 REFERENCE CALCULATIONS: CONVERSION FACILITY . . . . . . . A.37 DIFFUSION ENRICHMENT CHEMICAL RELEASE FACTORS. . . . . . A.43 DIFFUSION ENRIEHMENT RADIONUCLIDE RELEASE FACTORS • . . . A.46 REFERENCE CALCULATIONS: DIFFUSION ENRICHMENT FACILITY . • . A.47 $\mathrm{UO}_{2}$ FUEL FABRICATION CHEMICAL RELEASE FACTORS • • • • • A.58 $\mathrm{UO}_{2}$ FUEL FABRICATION RADIONUCLIDE RELEASE FACTORS • • • • A.60 REFERENCE CALCULATIONS: $\mathrm{UO}_{2}$ FUEL FABRICATION FACILITY • • • A. A MOX FUEL FABRICATION CHEMICAL RELEASE FACTORS . . . . . A.68 MOX FUEL FABRICATION RADIONUCLIDE RELEASE FACTORS . . . . . A.69 REFERENCE CALCULATIONS: MOX FUEL FABRICATION FACILITY . . • A.70

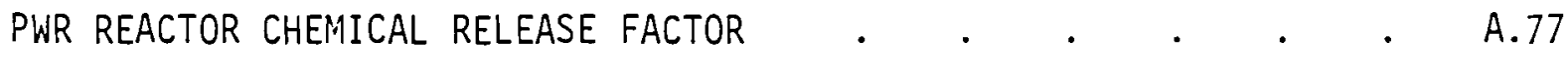
PWR REACTOR RADIONUCLIDE RELEASE FACTORS . . . . . A.80 REFERENCE CALCULATIONS: PWR REACTOR . . . . . . . . . A.85 
BWR REACTOR CHEMICAL RELEASE FACTORS . . . . . . A.109 BWR REACTOR RADIONUCLIDE RELEASE FACTORS. • • • • . A. . A10 REFERENCE CALCULATIONS: BWR REACTOR • • • • • • • A.115 REPROCESSING CHEMICAL RELEASE FACTORS . . . . . . . A. . A 133 REPROCESSING RADIONUCLIDE RELEASE FACTORS • • • • . . A.135 REFERENCE CALCULATIONS: REPROCESSING • • • • . . . . A.138 REFERENCES . . . . . . . . . . . . A.160 


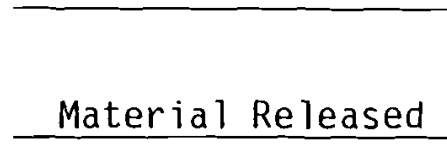
Air Borne

Uranium (natura 1)

Factor

Ref.

$3.9 \times 10^{-5}$
Reliability

Calc.
Factor

Thorium - 230

Radium - 226

$$
1.35 \times 10^{-10}
$$

Radon - 222

$2.5 \times 10^{-11}$

2

$1-1$

Radon Daughters:

$$
\text { Polonium - } 218
$$$$
\text { Lead - } 214
$$$$
\text { Bismuth - } 214
$$$$
\text { Polonium - } 214
$$$$
\text { Lead - } 210
$$

$$
\text { Polonium - } 210
$$

Carbon Monoxide (CO)

Nitrogen Oxides $\left(\mathrm{NO}_{\mathrm{x}}\right.$ )

Sulfur oxides $\left(\mathrm{SO}_{\mathrm{x}}\right)$

Aldehydes

$1.4 \times 10^{-14}$

$1.2 \times 10^{-13}$

$9.1 \times 10^{-14}$

2

$1-1$

$1.3 \times 10^{-20}$

$\begin{array}{ll}2 & 1-2 \\ 2 & 1-3\end{array}$

Nonradioact ive

Particulate

Hydrocarbons

Rock Dust

Vanadium (V)

Selenium (Se)

$4 \times 10^{-2}$

$3 \times 10^{-2}$

$1 \times 10^{-2}$

$3 \times 10^{-3}$

$1 \times 10^{-2}$

$1 \times 10^{-2}$

$9 \times 10^{-2}$

$2 \quad 1-4$

$2 \quad 1-4$

$2 \quad 1-4$

$2 \quad 1-4$

$1-4$

$1-5$

1-5

$1-5$

$1-5$

$2 \quad 1-5$

$2 \quad 1-5$

$2 \quad 1-5$

$1 \quad 1-6$




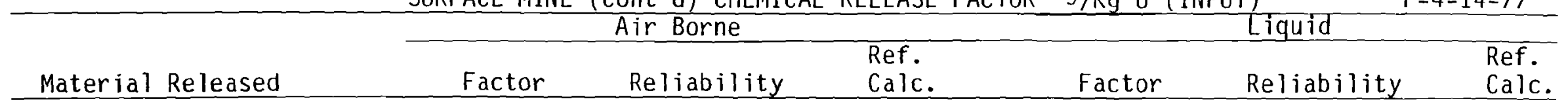

Mo lybedenum (Mo)

Arsenic (As)

Suspended Solids

Chloride (Cl)

Nitrate $\left(\mathrm{NO}_{3}\right)$

Sulfate $\left(\mathrm{SO}_{4}\right)$

Rock 


\begin{tabular}{|c|c|c|c|c|c|c|}
\hline \multirow[b]{3}{*}{ Material Released } & \multirow{2}{*}{\multicolumn{4}{|c|}{$\frac{\text { SURFACE MINE RADIONUCLIDE RELEASE FACTOR } \mathrm{Kg} / \mathrm{Kg} U \text { (INPUT) }}{\text { Air Borne }}$}} & \multirow{2}{*}{\multicolumn{2}{|c|}{ Liquid }} \\
\hline & & & & & & \\
\hline & Factor & Reliability & $\begin{array}{l}\text { Ref. } \\
\text { Calc. }\end{array}$ & Factor & Reliability & $\begin{array}{l}\text { Ref. } \\
\text { Calc. }\end{array}$ \\
\hline Uranium (natural) & $3.9 \times 10^{-5}$ & 2 & & & & \\
\hline *Uranium - 238 & $3.87 \times 10^{-5}$ & 2 & $1-7$ & & & \\
\hline *Uranium - 235 & $2.77 \times 10^{-7}$ & 2 & $1-7$ & & & \\
\hline *Uranium - 234 & $2.11 \times 10^{-9}$ & 2 & $1-7$ & & & \\
\hline \multicolumn{7}{|l|}{$\star$ Thor ium -230} \\
\hline *Radium - 226 & $1.35 \times 10^{-11}$ & 2 & $1-2$ & & & \\
\hline$\star$ Radon -222 & $2.5 \times 10^{-11}$ & 2 & $1-3$ & & & \\
\hline \multicolumn{7}{|l|}{ Radon Daughters: } \\
\hline Polonium - 218 & $1.4 \times 10^{-14}$ & 1 & $1-4$ & & & \\
\hline Lead - 214 & $1.2 \times 10^{-13}$ & 1 & $1-4$ & & & \\
\hline Bismuth - 214 & $9.1 \times 10^{-14}$ & 1 & $1-4$ & & & \\
\hline Polonium - 214 & $1.3 \times 10^{-20}$ & 1 & $1-4$ & & & \\
\hline \multicolumn{7}{|l|}{ *Lead -210} \\
\hline *Polonium - 210 & & & & & & \\
\hline
\end{tabular}

*Has Data Base In Computer Mode 1 
REFERENCE CALCULATIONS: SURFACE MINE (312 Operating Day/yr)

1-1. Uranium dust

from Rock dust calculations, $1-6$, and ore grade of $0.0015 \%$ u in ore, $0.000085 \%$ in waste rock

$$
\begin{aligned}
& \text { mining crushing waste rock } \\
& (111 \times 0.0015)+(30 \times 0.0015)+(457 \times 0.000085) \\
& =\frac{0.25 \mathrm{~kg}}{6350 \mathrm{~kg} / \text { day }}=3.9 \times 10^{-5} \frac{\mathrm{Kg} \mathrm{U} \text { dust }}{\mathrm{Kg} \mathrm{U}}
\end{aligned}
$$

1-2. Radium 226 radium in equilibrium with $U$ content

$=3.42 \times 10^{-7}$ fraction of $U$ content

$3.42 \times 10^{-7} \times 0.25 \frac{\mathrm{kg}}{\text { day }}$ from $1-1=8.555 \mathrm{E}-08 \frac{\mathrm{kg}}{\text { day }}$

Release factor $=\frac{8.555 \mathrm{E}-08}{6350}=1.35 \times 10^{-11} \frac{\mathrm{kg} \mathrm{Ra226}}{\mathrm{kg} \mathrm{U}}$

1-3. Rn-222 Surface mine (Ref. 35 p. 10)

$1961 \frac{\mathrm{Ci}}{\mathrm{yr}} \times \frac{\mathrm{yr}}{330 \text { day }} \times \frac{\mathrm{d}}{1722 \text { tons }} \times \frac{\text { ton }}{0.00115 \text { ton } \mathrm{U}_{3} \mathrm{O}_{8}}$

$\times \frac{\text { ton }}{0.9072 M T} \times \frac{\mathrm{t} \mathrm{U}_{3}{ }^{0} 8}{0.848 \operatorname{ton} U} \times \frac{6.5 \times 10^{-6} \mathrm{gRn} 222}{\mathrm{Ci} \mathrm{Rn222}}$

$\times \frac{\mathrm{kg}}{10^{3} \mathrm{~g}} \times \frac{\text { MTU }}{10^{3} \mathrm{~kg} \mathrm{U}}$

$=2.535 \times 10^{-11} \frac{\mathrm{kg} \mathrm{Rn} 222}{\mathrm{Kg} \mathrm{U}}$ 
REFERENCE CALCULATIONS: Surface Mine (contd)

1-4. Assuming secular equilibrium exists between radon-222 and its shorter half-1ived daughters then the daughter products vary inversely as the ratio of the radon-222 decay constant and the daughter product decay constant.

(from ref. calc. 1-3)

$2.54 \times 10^{-11} \frac{\mathrm{kg}^{222} \mathrm{Rn}}{\mathrm{kg} \mathrm{U}} \times \frac{2.10 \times 10^{-6}\left(\lambda-{ }^{222} \mathrm{Rn}\right)}{3.79 \times 20^{-3}\left(\lambda-{ }^{218} \mathrm{Po}\right)}=1.41 \times 10^{-14} \frac{\mathrm{kg}^{218} \mathrm{Po}_{0}}{\mathrm{~kg} \mathrm{U} \text { (input) }}$

(from ref. cal. 1-3)

$2.54 \times 10^{-11} \frac{\mathrm{kg}^{222} \mathrm{Rn}}{\mathrm{kg} \mathrm{U}} \times \frac{2.10 \times 10^{-6}\left(\lambda-^{222} \mathrm{Rn}\right)}{4.31 \times 10^{-4}\left(\lambda-{ }^{214} \mathrm{~Pb}\right)}=1.24 \times 10^{-13} \frac{\mathrm{kg}^{214} \mathrm{~Pb}}{\mathrm{~kg} \mathrm{U} \text { (input) }}$

(from ref. calc. 1-3)

$2.54 \times 10^{-11} \frac{\mathrm{kg}^{222} \mathrm{Rn}}{\mathrm{kg} \mathrm{U}} \times \frac{2.10 \times 10^{-6}\left(\lambda-{ }^{222} \mathrm{Rn}\right)}{5.86 \times 10^{-4}\left(\lambda-{ }^{214} \mathrm{Bi}\right)}=9.10 \times 10^{-14} \frac{\mathrm{kg}^{214} \mathrm{Bi}}{\mathrm{kg} \mathrm{U} \text { (input) }}$

(from ref. calc. 1-3)

$2.54 \times 10^{-11} \frac{\mathrm{kg}^{222} \mathrm{Rn}}{\mathrm{kg} \mathrm{U}} \times \frac{2.10 \times 10^{-6}\left(\lambda-^{222} \mathrm{Rn}\right)}{4.23 \times 10^{3}\left(\lambda-{ }^{214} \mathrm{Po}\right)}=1.26 \times 10^{-20} \frac{\mathrm{kg}^{214} \mathrm{Po}_{0}}{\mathrm{~kg} \mathrm{U} \text { (input) }}$ 


\section{REFERENCE CALCULATIONS: SURFACE MINE (contd)}

1-5. Bas is for the following numbers is 6500 gallons of diesel fuel per day at the reference

surface mine and EPA emission factors, $(1, \mathrm{pp} .3 .2 .7-2,3)$. Mine throughput is $6350 \mathrm{kgU} / \mathrm{day}$.

CO

$$
\frac{85 \mathrm{lb}}{10^{3} \mathrm{gal}} \times 6.5 \times 10^{3} \frac{\mathrm{gal}}{\mathrm{day}} \frac{\mathrm{kg}}{2.2 \mathrm{lb}} \times \frac{1}{6350} \frac{\mathrm{day}}{\mathrm{kgU}}=4.0 \mathrm{E}-2
$$

NO $x$

$$
\frac{55 \mathrm{lb}}{10^{3} \mathrm{gal}} \times 6.5 \times 10^{3} \mathrm{gal} \frac{\mathrm{kg}}{2.2 \mathrm{lb}} \times \frac{1}{6350} \frac{\mathrm{day}}{\mathrm{kgU}}=2.6 \mathrm{E}-2
$$

$\mathrm{SO}_{\mathrm{x}}$

$$
\frac{31.2 \mathrm{lb}}{10^{3} \mathrm{gal}} \times 6.5 \times 10^{3} \mathrm{gal} \frac{\mathrm{kg}}{2.2 \mathrm{lb}} \times \frac{1}{6350} \frac{\text { day }}{\mathrm{kgU}}=1.45 \mathrm{E}-2
$$

Aldehydes $\frac{6.8 \mathrm{lb}}{10^{3} \mathrm{gal}} \times 6.5 \times 10^{3} \mathrm{gal} \frac{\mathrm{kg}}{2.2 \mathrm{lb}} \times \frac{1}{6350 \mathrm{kgU}} \frac{\text { day }}{\mathrm{kgU}}=3.2 \mathrm{E}-3$

Particulate $\frac{25 \mathrm{lb}}{10^{3} \mathrm{gal}} \times 6.5 \times 10^{3} \mathrm{gal} \frac{\mathrm{kg}}{2.2 \mathrm{lb}} \times \frac{1}{6350} \frac{\mathrm{day}}{\mathrm{kgU}}=1.2 \mathrm{E}-2$

Hydrocarbon $\frac{30 \mathrm{lb}}{10^{3} \mathrm{gal}} \times 6.5 \times 10^{3} \mathrm{gal} \frac{\mathrm{kg}}{2.2 \mathrm{lb}} \times \frac{1}{6350 \mathrm{kgU}}=1.4 \mathrm{E}-2$

\section{1-6. Rock Dust}

Mining (34, p. 57 Table 21)

$$
\begin{aligned}
& 750 \frac{\mathrm{pCi}}{\mathrm{sec}} \times \frac{2337}{3000} \times \frac{0.0025}{0.0015} \times \frac{2.97 \mathrm{E}+6 \mathrm{~g} \mathrm{U}}{10^{12} \mathrm{pCi}} \times \frac{\mathrm{g} \text { dust }}{0.0015 \mathrm{~g} \mathrm{U}} \times \frac{\mathrm{kg}}{10^{3} \mathrm{~g}} \times \frac{8.64 \mathrm{E}+4 \mathrm{sec}}{\text { day }} \\
& \begin{array}{ccc}
\underset{\text { production rate }}{\text { ratio }} & \text { grade } & \text { gram to curie } \\
\text { ratio } & \text { conversion }
\end{array}
\end{aligned}
$$


REFERENCE CALCULATIONS: SURFACE MINE (contd)

$$
\begin{aligned}
& x \frac{16 \mathrm{hr}}{\frac{d a y}{24 \mathrm{hr}}}=111 \frac{\mathrm{kg}}{\mathrm{day}} \\
& \frac{111 \frac{\mathrm{kg}}{\mathrm{day}}}{6350 \frac{\mathrm{kg} \mathrm{U}}{\text { day }}}=1.75 \mathrm{E}-2 \frac{\mathrm{kg}}{\mathrm{kg} \mathrm{U}}
\end{aligned}
$$

(34, p. 29)

$$
\begin{array}{ll}
\text { blasting }-0.95^{\star} & 111=105 \frac{\mathrm{kg}}{\mathrm{day}}=1.65 \mathrm{E}-2 \frac{\mathrm{kg}}{\mathrm{kg} \mathrm{U}} \\
\text { haulage }=0.05^{\star} & 111=6 \frac{\mathrm{kg}}{\text { day }}=9.45 \mathrm{E}-4 \frac{\mathrm{kg}}{\mathrm{kg} \mathrm{U}}
\end{array}
$$

Crushing (primary on ly)

primary crushing contributes about $2 \%$ of the fine dust from primary, secondary and tertiary crushing (1, p. 8.20-1). For conservatism, assume 5\% is from primary crushing. $(1$, p. 8.20-1) (34, p. 57 Table 21)

$0.05 \times 4044 \frac{\mathrm{pCi}}{\mathrm{sec}} \times \frac{2337}{3000} \times \frac{0.25 \%}{0.15 \%} \times \frac{2.97 \mathrm{E}+6 \mathrm{gU}}{10^{12} \mathrm{pCi}}$

$\times \frac{\mathrm{g} \text { dust }}{0.0015 \mathrm{~g} \mathrm{U}} \times \frac{\mathrm{kg}}{10^{3} \mathrm{~g}} \times 8.64 \mathrm{E}+4 \frac{\mathrm{sec}}{\text { day }} \times \frac{16 \mathrm{hr}}{24 \mathrm{hr}}=30 \frac{\mathrm{kg}}{\text { day }}$ $\frac{30 \frac{\mathrm{kg} \text { dust }}{\text { day }}}{6350 \frac{\mathrm{kg} \mathrm{U}}{\text { day }}}=4.710^{-3} \frac{\mathrm{kg} \text { dust }}{\mathrm{kg} \mathrm{U}}$ 
REFERENCE CALCULATIONS: SURFACE MINE (contd)

Waste Rock Pile

assume wind speed $=16 \mathrm{~km} / \mathrm{hr}=4.4 \mathrm{~m} / \mathrm{sec}$ (p. 5-19, site description).

(34, p. 57 Table 22, interpolated) tails $0.01 \% \mathrm{U}_{3} 0_{8}$

$9.0 \times 10^{-4} \frac{\mathrm{g} \mathrm{U}}{\mathrm{sec}} \times \frac{\mathrm{g} \text { dust }}{0.000085 \mathrm{~g} \mathrm{U}} \times 86,400 \frac{\mathrm{sec}}{\mathrm{day}} \times \frac{\mathrm{kg}}{10^{3} \mathrm{~g}} \times \frac{1}{2}=457 \frac{\mathrm{kg}}{\text { day }}$

(size of waste rock pile average over mine life)

$\frac{457 \frac{\mathrm{kg}}{\text { day }}}{6350 \frac{\mathrm{kg} \mathrm{U}}{\text { dày }}}=7.20 \times 10^{-2} \frac{\mathrm{kg} \mathrm{dust}}{\mathrm{kg} \mathrm{U}}$

Total release factor $=\frac{111 \mathrm{~kg}+30 \mathrm{~kg}+457 \mathrm{~kg}}{6350 \mathrm{~kg} \mathrm{U}}=9.4 \times 10^{-2} \frac{\mathrm{kg} \text { rock dust }}{\mathrm{kg} \mathrm{U}}$

$\stackrel{D}{\sim}$

1-7. (from ref. calc. 1-1) (4, p. B-35)

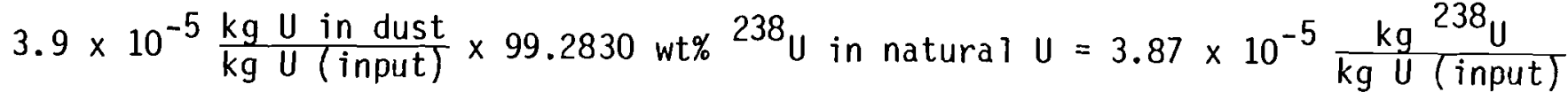

(from ref. calc. 1-1) (4, p. B-35)

$3.9 \times 10^{-5} \frac{\mathrm{kg} \mathrm{U} \text { in dust }}{\mathrm{kg} U \text { (input) }} \times 0.7110 \%{ }^{235} \mathrm{U}$ in natural $U=2.77 \times 10^{-7} \frac{\mathrm{kg}^{235} \mathrm{U}}{\mathrm{kg} U \text { (input) }}$

(from ref. calc. 1-1) (4, p. B-35)

$3.9 \times 10^{-5} \frac{\mathrm{kg} U \text { in dust }}{\mathrm{kg} \mathrm{U} \text { (input) }} \times 0.0054 \%{ }^{234} \mathrm{U}$ in natural $\mathrm{U}=2.11 \times 10^{-9} \frac{\mathrm{kg}{ }^{234} \mathrm{U}}{\mathrm{kg} \text { (input) }}$ 
UNDERGROUND MINE CHEMICAL RELEASE FACTOR $\mathrm{Kg} / \mathrm{Kg} \mathrm{U}$ (INPUT)

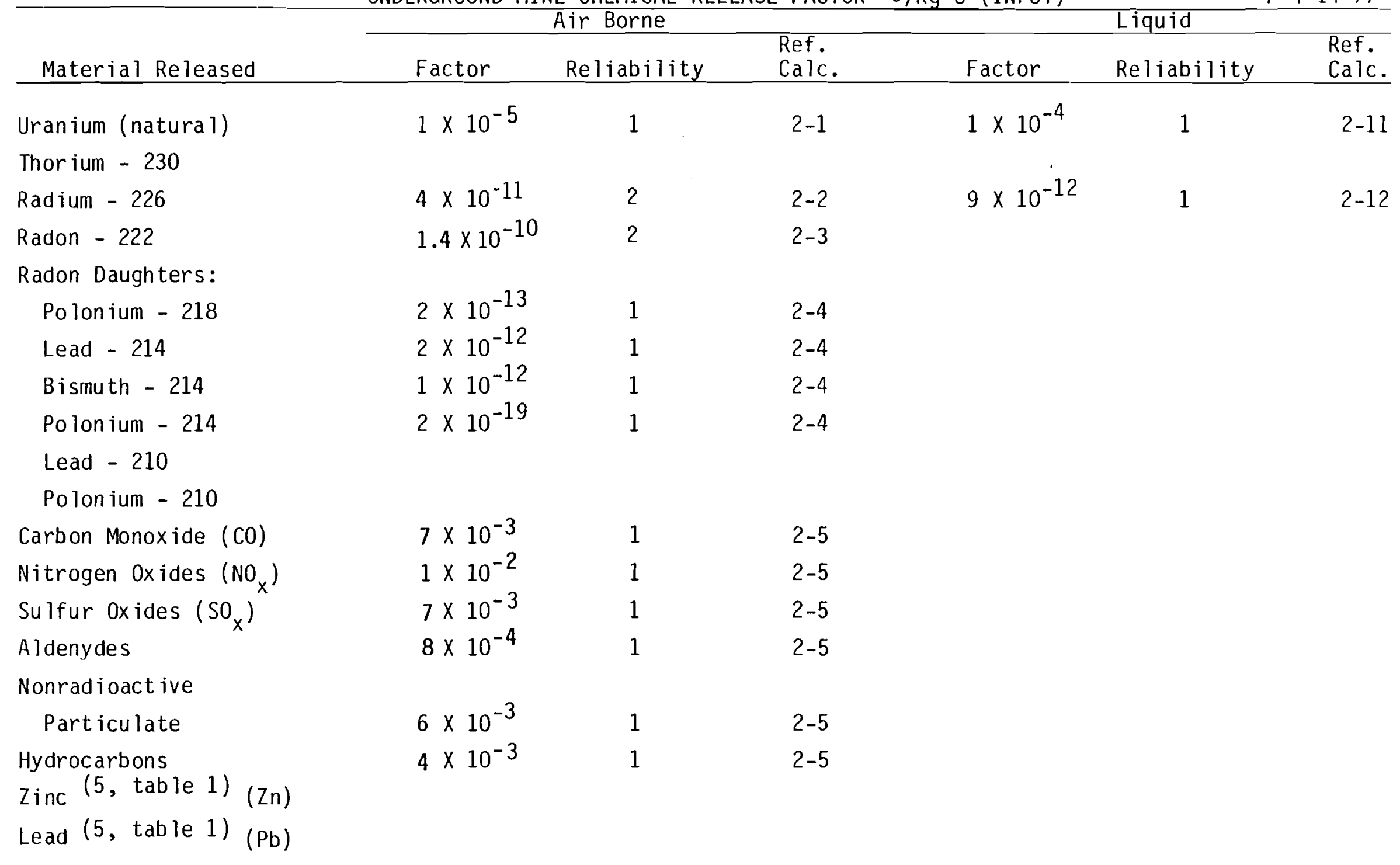




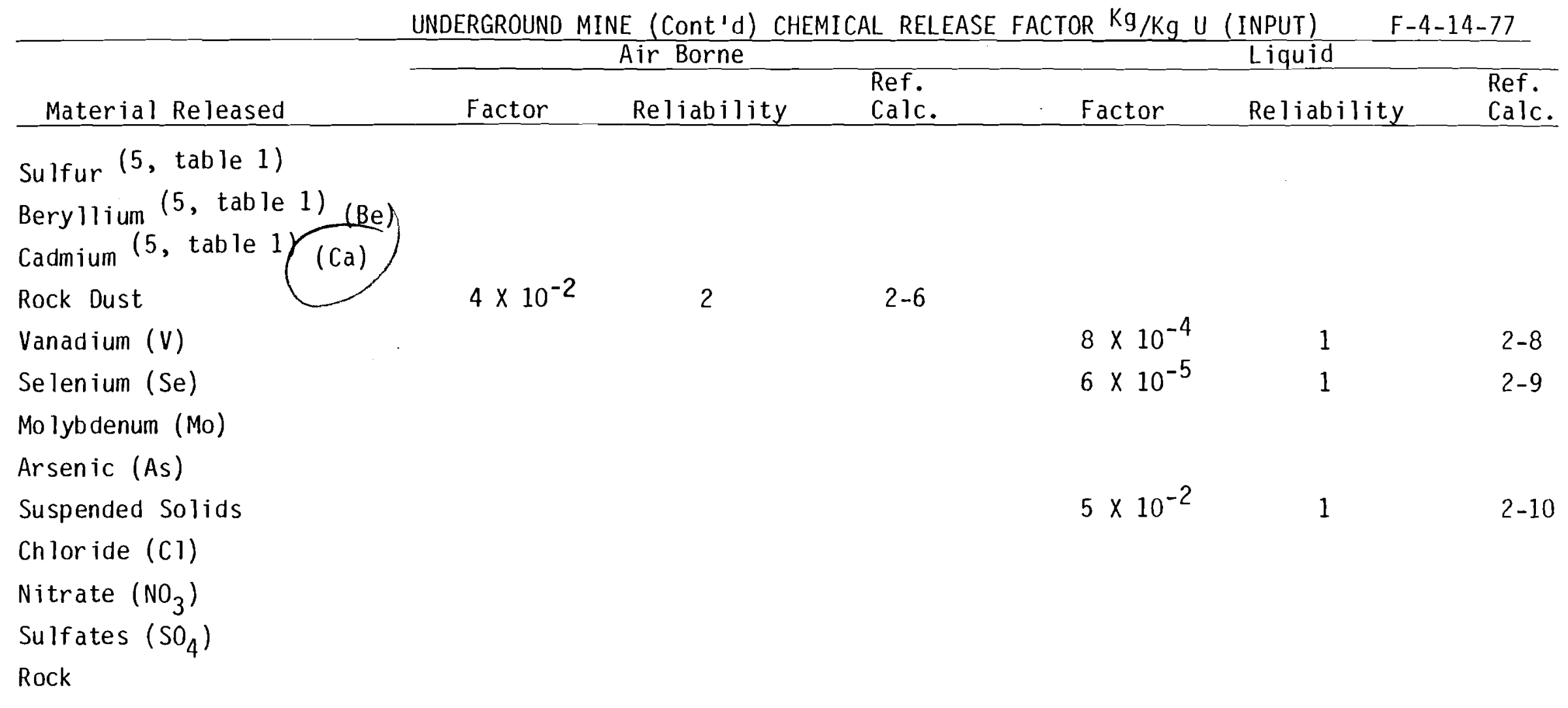




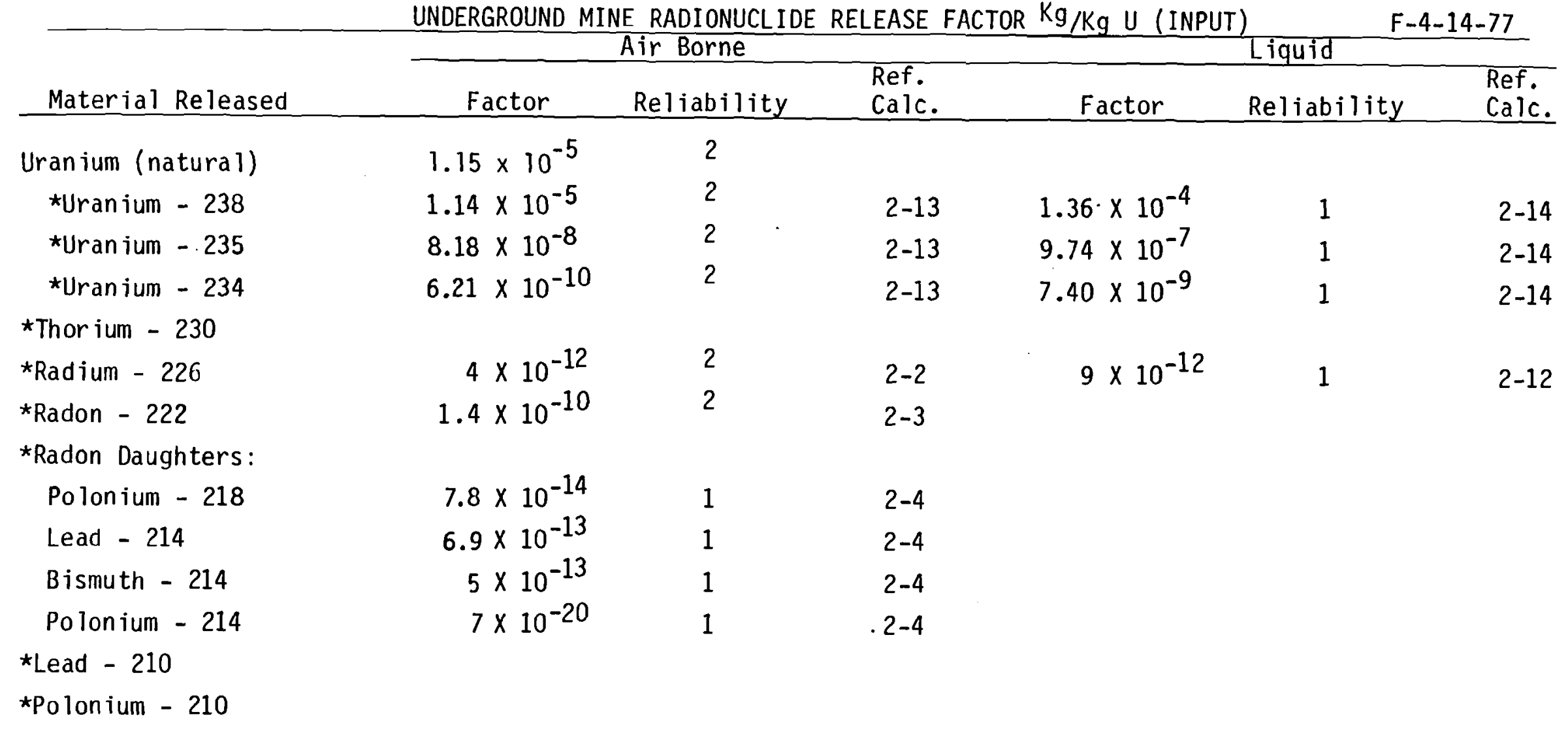

* Has Data Base In Computer Model 


\section{REFERENCE CALCULATIONS: UNDERGROUND MINE (365 Operating Days/yr)}

2-1. Uranium Dust

from Rock dust calculations, 2-6. and grade assumption: $0.15 \% U$ in ore; $0.0085 \% \mathrm{U}$ in waste rock

$$
\begin{aligned}
& \text { hauling crushing waste rock } \\
& (6 \times 0.0015)+(30 \times 0.0015)+(229 \times 0.000085) \\
& \frac{0.073 \mathrm{~kg} \mathrm{U}}{6350 \mathrm{~kg} \mathrm{U}}=1.15 \times 10^{-5} \frac{\mathrm{kg} \mathrm{U} \text { dust }}{\mathrm{kg} \mathrm{U}}
\end{aligned}
$$

2-2. Radium 226

radium in equilibrium with natural uranium $=3.42 \times 10^{-7}$ fraction of $U$ content

2-3. Radium 222 (36, p. 19)

$\left(15.9 \frac{\mathrm{Ci}}{\operatorname{ton} \mathrm{U}_{3} 0_{8}}+0.8 \frac{\mathrm{Ci}}{\operatorname{ton} \mathrm{U}_{3} 0_{8}}\right) \times \frac{\mathrm{t}}{0.9072 \mathrm{MT}}$

from vents above ground sources

$\times \frac{\text { ton } \mathrm{U}_{3} \mathrm{O}_{8}}{0.848 \text { ton } \mathrm{U}} \times \frac{6.5 \times 10^{-6} \mathrm{gRn} 222}{\mathrm{Ci} \operatorname{Rn} 222} \times \frac{\mathrm{kg}}{10^{3} \mathrm{~g}} \times \frac{\mathrm{MT}}{10^{3} \mathrm{~kg}}=1.41 \times 10^{-10 \mathrm{~kg} \mathrm{Rn} 222}$

2-4. Assuming secular equilibrium exists between radon-222 and its shorter half-lived daughters then the daughter products vary inversely as the ratio of the radon-222 decay constant and the daughter product decay constant. (from ref. calc. 2-3) 


\section{REFERENCE CALCULATIONS: UNDERGROUND MINE (contd)}

$1.41 \times 10^{-10} \frac{\mathrm{kg}{ }^{222} \mathrm{Rn}}{\mathrm{kg} \mathrm{U}} \times \frac{2.10 \times 10^{-6}\left(\lambda-{ }^{222} \mathrm{Rn}\right)}{3.79 \times 10^{-3}\left(\lambda-{ }^{218} \mathrm{Po}\right)}=7.78 \times 10^{-14} \frac{\mathrm{kg}^{218} \mathrm{Po}}{\mathrm{kgU} \text { (input) }}$

$1.41 \times 10^{-10} \frac{\mathrm{kg}^{222} \mathrm{Rn}}{\mathrm{kg} \mathrm{U}} \times \frac{2.10 \times 10^{-6}\left(\lambda-{ }^{222} \mathrm{Rn}\right)}{4.31 \times 10^{-4}\left(\lambda-{ }^{214} \mathrm{~Pb}\right)}=6.85 \times 10^{-13} \frac{\mathrm{kg}^{214} \mathrm{~Pb}}{\mathrm{kgU} \text { (input) }}$

$1.41 \times 15^{-10} \frac{\mathrm{kg}}{\mathrm{kg} U} \times \frac{2.10 \times 10^{-6}\left(\lambda \lambda^{222} \mathrm{Rn}\right)}{5.86 \times 10^{-4}\left(\lambda_{-}{ }^{214} \mathrm{Bi}\right)}=5.04 \times 10^{-13} \frac{\mathrm{kg}{ }^{214} \mathrm{Bi}}{\mathrm{kg} U \text { (input) }}$

$1.41 \times 10^{-10} \frac{\mathrm{kg}^{222} \mathrm{Ra}}{\mathrm{kg}} \times \frac{2.10 \times 10^{-6}\left(\lambda_{-}^{222} \mathrm{Rn}\right)}{4.23 \times 10^{3}\left(\lambda^{214} \mathrm{Po}\right)}=7.00 \times 10^{-20} \frac{\mathrm{kg}^{214} \mathrm{Po}}{\mathrm{kg} U \text { (input) }}$

2-5. The release factors for combustion products from diesel exhaust are taken as half the quantity from the surface mine plus the assumption that catalytic exhaust purities are used (37).
$(37$, p. 5)
co
$\frac{251}{2} \mathrm{~kg} \times 0.35=44 \frac{\mathrm{kg}}{\text { day }}$
$\mathrm{RF}=\frac{44}{6350}=6.9 \times 10^{-3}$
${ }^{\mathrm{NO}} \mathrm{X}$
$\frac{163}{2} \mathrm{~kg} \times 0.35=82 \frac{\mathrm{kg}}{\text { day }}$
$R F=\frac{82}{6350}=1.3 \times 10^{-2}$
$\mathrm{SO}_{\mathrm{X}}$
$\frac{92}{2} \mathrm{~kg} \times 0.35=46 \frac{\mathrm{kg}}{\text { day }}$
$R F=\frac{46}{6350}=7.2 \times 10^{-3}$
(37, p. 8$)$
Aldehydes
$\frac{20}{2} \mathrm{~kg} \times 0.5=5 \frac{\mathrm{kg}}{\text { day }}$
$\mathrm{RF}=\frac{5}{6350}=7.9 \times 10^{-4}$
Particulate $\quad \frac{74}{2} \mathrm{~kg} \times 0.5=37 \frac{\mathrm{kg}}{\text { day }}$
$R F=\frac{37}{6350}=5.8 \times 10^{-3}$
$(37$, p. 5$)$
Hydrocarbon $\quad \frac{87}{2} \mathrm{~kg} \times 0.6=26 \frac{\mathrm{kg}}{\text { day }}$
$R F=\frac{26}{6350}=4.1 \times 10^{-3}$ 


\section{REFERENCE CALCULATIONS: UNDERGROUND MINE (contd)}

2-6. Rock dust

(from calculation 1-6, with no contribution from blasting and only $1 / 2$ as much waste rock)

$\begin{array}{ccc}\text { hauling } & \text { crushing } & \text { Waste rock } \\ 6 \frac{\mathrm{kg}}{\mathrm{day}} & 30 \frac{\mathrm{kg}}{\text { day }} & 229 \frac{\mathrm{kg}}{\text { day }}\end{array}$

$\frac{265 \mathrm{~kg} \mathrm{Rock} \mathrm{dust}}{6350 \mathrm{~kg} \mathrm{U}}=4.17 \mathrm{E}-2 \frac{\mathrm{kg} \text { Rock Dust }}{\mathrm{kg} \mathrm{U}}$

2-7. Kerr-McGee Ambrosia Lake mines (sections 19,30W, 35, 36) are assumed to be the only mines in the reference facility that discharge water to the environment (3, p. 23). Of this water only $25 \%$ is assumed to be discharged to surface water (no real basis for this number - arbitrary) following ion exchange at the mines.

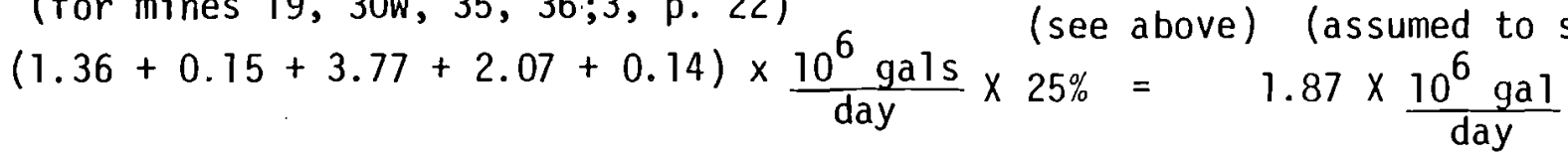

(average of values for mines

2-8. $\left(\frac{0.7+0.6+0.8+0.9+0.6}{5}\right) \frac{\mathrm{mg} \mathrm{V}}{1 \mathrm{iter}} \times \frac{3.7851 \mathrm{titer}}{\mathrm{gal}} \times 1.87 \times 10^{6} \frac{\mathrm{gal}}{\text { day }} \times \frac{\mathrm{kg}}{10^{6} \mathrm{mg}}=5.10 \frac{\mathrm{kg} \mathrm{V}}{\text { day }}$

(from ref. calc. 2-7)

$$
\therefore \frac{5.10 \mathrm{~kg} \mathrm{~V} / \text { day }}{6351 \mathrm{~kg} \mathrm{U} / \text { day }}=8.03 \times 10^{-4} \frac{\mathrm{kg} \mathrm{V}}{\mathrm{kgU} \text { (input) }}
$$

2-9. Assumed an average concentration for Se at $0.05 \mathrm{mg} / 1$ iter after referring to (3, p.22).

$$
\begin{aligned}
& \text { (see above) } \\
& 0.05 \frac{\mathrm{mg} \mathrm{Se}}{1 \text { iter }} \times \frac{3.785 \text { liter }}{\text { gal }} \times 1.87 \times 10^{6} \frac{\mathrm{gal}}{\text { day }} \times \frac{\mathrm{kg}}{10^{6} \mathrm{mg}}=3.54 \times 10^{-1} \frac{\mathrm{kg} \mathrm{Se}}{\text { day }}
\end{aligned}
$$


REFERENCE CALCULATIONS: UNDERGROUND MINE (contd)

$$
\begin{aligned}
& \therefore \frac{3.54 \times 10^{-1} \mathrm{~kg} \mathrm{Se} / \text { day }}{6351 \mathrm{~kg} U / \text { day }}=5.57 \times 10^{-5} \frac{\mathrm{kg} \mathrm{Se}}{\mathrm{kg} U \text { (input) }} \\
& \left(\begin{array}{l}
\text { average of values for mines } \\
19,30 \mathrm{~W}, 35,36 ; 3, \mathrm{p} .22)
\end{array}\right. \\
& \text { 2-10. }\left(\frac{22+16+100+38+29}{5}\right) \frac{\text { mg suspended solids }}{1 \text { iter }} \times \frac{3.785 \text { liter }}{\text { gal }} \times
\end{aligned}
$$

(from ref. calc. 2-7)

$$
\begin{gathered}
1.87 \times 10^{6} \frac{\mathrm{gal}}{\text { day }} \times \frac{\mathrm{kg}}{10^{6} \mathrm{mg}}=2.90 \times 10^{2} \frac{\mathrm{kg} \text { suspended solids }}{\text { day }} \\
\therefore \frac{2.90 \times 10^{2} \mathrm{~kg} \text { suspended sol ids } / \text { day }}{6351 \mathrm{~kg} \mathrm{U} / \mathrm{day}}=\frac{4.57 \times 10^{-2} \mathrm{~kg} \text { suspended solids }}{\mathrm{kg} \mathrm{U} \text { (input) }}
\end{gathered}
$$

2-11. Assuming that uranium recovery of the ion exchange treatment of groundwater and surface water is $98 \%$ efficient then the following is the calculation of the amount of uranium in the surface water discharge.

(average of values for mines

$19,30 \mathrm{~W}, 35,36 ; 3, \mathrm{p.} 22$ ) (from ref. calc. 2-7)

$\left(\frac{6.2+0.23+19+3.0+2.4}{5}\right) \frac{\mathrm{mg} \mathrm{U}}{1 \text { iter }} \times \frac{3.7851 \text { iters }}{\text { gal }} \times 1.87 \times 10^{6} \frac{\mathrm{gal}}{\text { day }} \times \frac{\mathrm{kg}}{10^{6} \mathrm{mg}} \times$

(amount remaining after

ion exchange, see above)

$$
2 \%
$$

$$
=8.73 \times 10^{-1} \frac{\mathrm{kg} \mathrm{U}}{\mathrm{day}}
$$

$$
\therefore \frac{8.73 \times 10^{-1} \mathrm{~kg} \mathrm{U} / \text { day }}{6351 \mathrm{~kg} \mathrm{U/ \text {day }}}=1.37 \times 10^{-4} \frac{\mathrm{kg} \mathrm{U}}{\mathrm{kgU} \text { (input) }}
$$




\section{REFERENCE CALCULATIONS: UNDERGROUND MINE (contd)}

(average of values for mines

$19,30 \mathrm{~W}, 35,36 ; 3$, p.22) (from ref. calc. 2-7)

2-12. $\left(\frac{163+9.3+51+13 i+65}{5}\right) \times \frac{10^{-12} \mathrm{Ci}}{7 i \text { ter }} \times \frac{3.785 \text { liters }}{\text { gal }} \times 1.87 \times 10^{6} \frac{\mathrm{gal}}{\mathrm{day}} \times \frac{\mathrm{g}}{.99 \mathrm{Ci}} \times \frac{\mathrm{kg}}{10^{3} \mathrm{~g}} \times$

(assumed amount of $22 \epsilon_{\mathrm{Ra}}$ released

after barium chloride treatment)

$10 \%=6.00 \times 10^{-8} \frac{\mathrm{kg}{ }^{226} \mathrm{Ra}}{\text { day }} \quad \therefore \frac{6.00 \times 10^{-8} \mathrm{~kg}^{226} \mathrm{Ra} / \text { day }}{6351 \mathrm{~kg} \mathrm{U/day}}=9.45 \times 10^{-12} \frac{\mathrm{kg}{ }^{226} \mathrm{Ra}}{\mathrm{kg} \mathrm{U} \text { (input) }}$

(from ref. calc. 2-1)

2-13. $1.15 \times 10^{-5} \frac{\mathrm{kg} U \text { dust }}{\mathrm{kg} U \text { (input) }} \times 99.2830 \%{ }^{238} U$ in nat' $1 U=1.14 \times 10^{-5} \frac{\mathrm{kg}^{238} \mathrm{U}}{\mathrm{kg} U \text { (input) }}$

(from ref. calc. 2-1)

$1.15 \times 10^{-5} \frac{\mathrm{kg} U \text { dust }}{\mathrm{kgU} U \text { (input) }} \times 0.7110 \%{ }^{235} \mathrm{U}$ in nat' $1 U=8.18 \times 10^{-8} \frac{\mathrm{kg}^{235} \mathrm{U}}{\mathrm{kg} \mathrm{U} \text { (input) }}$

(from ref. calc. 2-1) (4, p. B-35)

$1.15 \times 10^{-5} \frac{\mathrm{kg} U \text { dust }}{\mathrm{kg} U \text { (input) }} \times 0.0054 \%{ }^{234} \mathrm{U}$ in nat' $1 \mathrm{U}=6.21 \times 10^{-10} \frac{\mathrm{kg}}{\mathrm{kg} U \text { (input) }}$

(from ref. calc. 2-11) (4, p. B-35)

2-14. $1.37 \times 10^{-4} \frac{\mathrm{kg} \mathrm{U}}{\mathrm{kgU} \text { (input) }} \times 99.2830 \%{ }^{238} \mathrm{U}$ in nat' $1 \mathrm{U}=1.36 \times 10^{-4} \frac{\mathrm{kg}^{238} \mathrm{U}}{\mathrm{kgU} \text { (input) }}$

(from ref. calc. 2-11) (4, p. B-35)

$1.37 \times 10^{-4} \frac{\mathrm{kg} \mathrm{U}}{\mathrm{kgU} \text { (input) }} \times 0.7110 \%{ }^{235} \mathrm{U}$ in nat' $1 \mathrm{U}=9.74 \times 10^{-7} \frac{\mathrm{kg}^{235} \mathrm{U}}{\mathrm{kg} \mathrm{U} \text { (input) }}$ 
REFERENCE CALCULATIONS: UNDERGROUND MINE (contd)

(from ref. calc. 2-11) (4, p. B-35)

$1.37 \times 10^{-4} \frac{\mathrm{kg} \mathrm{U}}{\mathrm{kg} \mathrm{U} \text { (input) }} \times 0.0054 \%{ }^{234} \mathrm{U}$ in nat' $1 \mathrm{U}=7.40 \times 10^{-9} \frac{\mathrm{kg}}{\mathrm{kg} \mathrm{U} U \text { (input) }}$ 


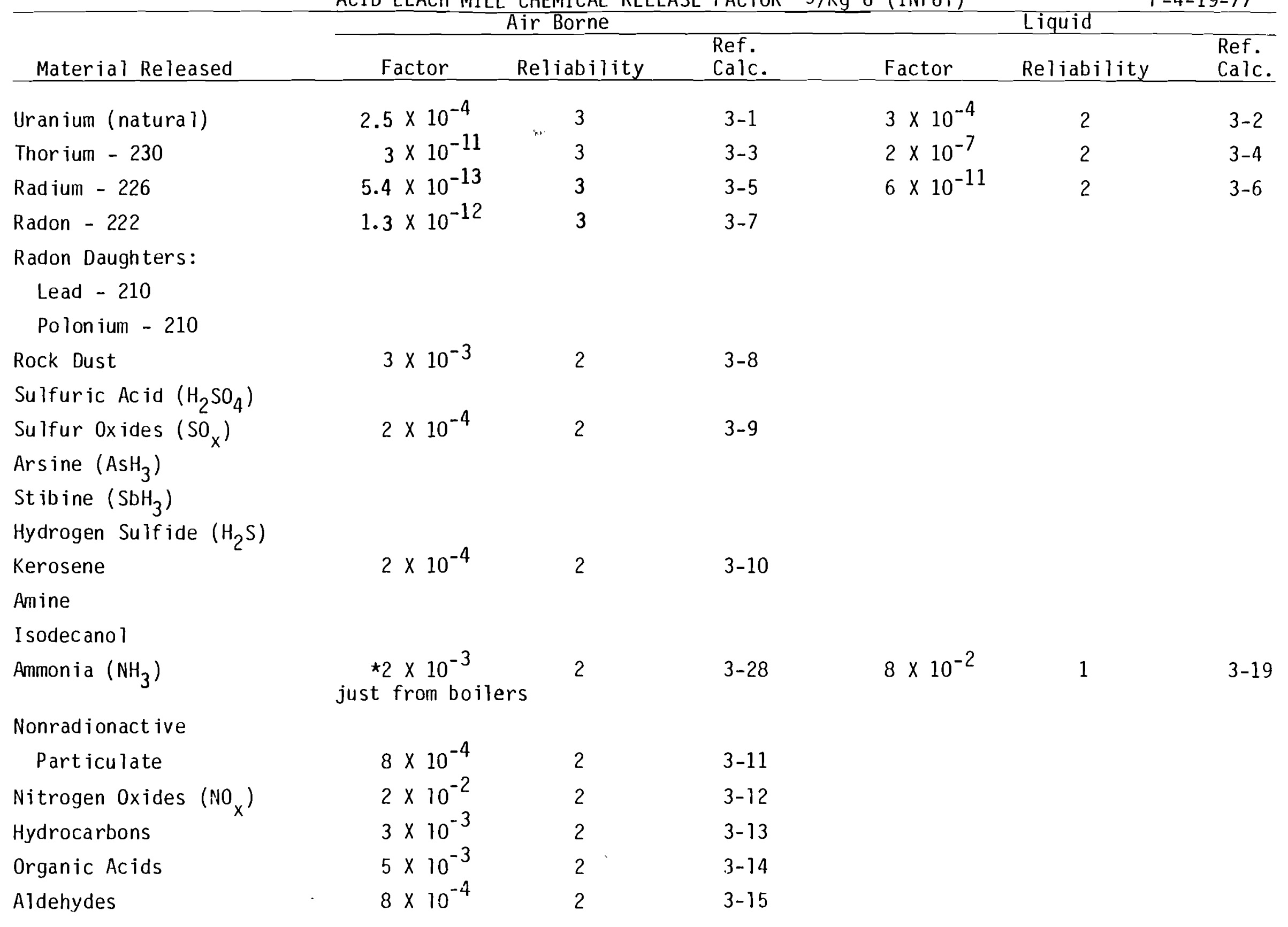

* ; Data Base in Computer Model 
ACID LEACH MILL (Cont'd) CHEMICAL RELEASE FACTOR $\mathrm{Kg} / \mathrm{Kg} \mathrm{U}$ (INPUT)

$F-4-19-77$

\begin{tabular}{|c|c|c|c|c|c|c|}
\hline \multirow[b]{2}{*}{ Material Released } & \multicolumn{3}{|c|}{ Air Borne } & \multicolumn{3}{|c|}{ Liquid } \\
\hline & Factor & Reliability & $\begin{array}{l}\text { Ref. } \\
\text { Calc. }\end{array}$ & Factor & Reliability & $\begin{array}{l}\text { Ref. } \\
\text { Calc. }\end{array}$ \\
\hline
\end{tabular}

$\begin{array}{llrr}\text { Calcium (Ca) } & 8 \times 10^{-2} & 1 & 3-16 \\ \text { Iron (Fe) } & 2 \times 10^{-1} & 1 & 3-17 \\ \text { Aluminum ( } \mathrm{AL}) & 3 \times 10^{-1} & 1 & 3-18 \\ \text { Sodium (Na) } & 3 \times 10^{-2} & 1 & 3-20 \\ \text { Arsenic (As) } & 3 \times 10^{-5} & 1 & 2-21 \\ \text { Fluoride (F) } & 8 \times 10^{-4} & 1 & 3-22 \\ \text { Vanadium (V) } & 2 \times 10^{-5} & 1 & 3-23 \\ \left.\text { Sulfate ( } \mathrm{SO}_{4}\right) & 5 & 1 & 3-24 \\ \text { Chloride (Cl) } & 5 \times 10^{-2} & 1 & 3-25 \\ \left.\text { Nitrates ( } \mathrm{NO}_{3}\right) & & \\ \text { Copper (Cu) } & & \\ \text { Beryllium (Be) } & & & \\ \text { Manganese (Mn) } & & & \\ \text { Magnesium (Mg) } & & & \\ \text { Lead (Pb) } & & & \\ \text { Boron (B) } & & & \end{array}$




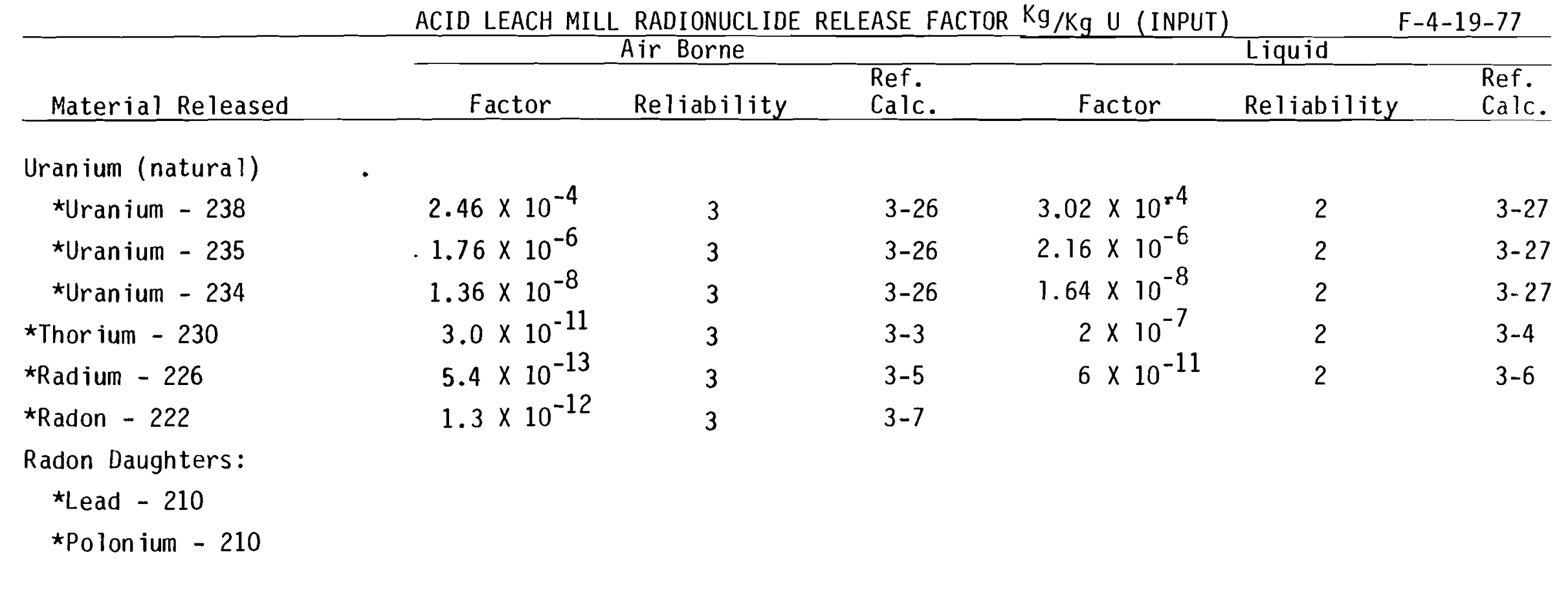

*Has Data Base In Computer Model 


\section{REFERENCE CALCULATIONS: MILLING FACILITY ( 365 0perating Days/Yr)}

3-1. $U$ input to the milling facility:

$\frac{2000 \text { short tons ore }}{\text { day }} \times \frac{907.2 \mathrm{~kg}}{\text { short ton }} \times 0.0015 \mathrm{U}$ in ore $=2722 \frac{\mathrm{kg} \mathrm{U}}{\text { day }}$

$U$ output from the milling facility: (38, p. 5-11)

\begin{tabular}{ll} 
Crusher & $2.37 \mu \mathrm{Ci} /$ day \\
Fine ore bin & 0.12 \\
Leach & 0.71 \\
Ore sampling & 0.10 \\
$\begin{array}{ll}\text { Yellow cake } \\
\text { dryer }\end{array}$ & 226 \\
\cline { 2 - 2 } & $229 . \mu \mathrm{Ci} / \mathrm{da}$
\end{tabular}

$229 \frac{\mu C i}{d a} \times 2.95 \times 10^{6} \frac{\mathrm{g}}{\mathrm{Ci}} \times \frac{\mathrm{Ci}}{10^{6} \mu \mathrm{Ci}} \times \frac{\mathrm{kg}}{10^{3} \mathrm{~g}}=0.68 \frac{\mathrm{kg}}{\mathrm{da}}$

$R F=\frac{0.68 \mathrm{~kg}}{2722 \mathrm{~kg}}=2.48 \times 10^{-4} \frac{\mathrm{kg} \mathrm{U}}{\mathrm{kg} \mathrm{U} \text { input }}$ 
REFERENCE CALCULATIONS: Milling Facility (cont'd)

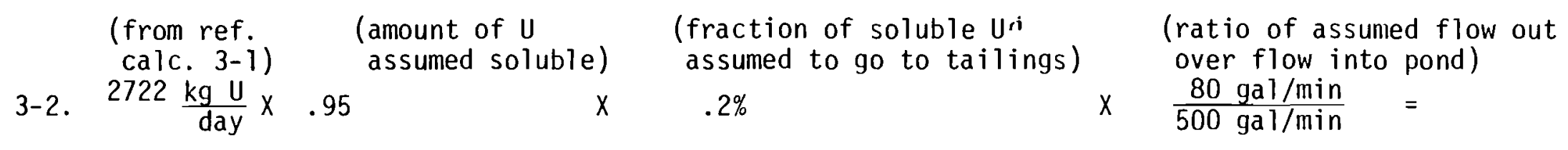

$$
\begin{aligned}
& 8.27 \times 10^{-1} \frac{\mathrm{kg} \mathrm{U}}{\text { day }} \text { (10ss due to seepage) } \\
& \therefore \frac{8.27 \times 10^{-1} \mathrm{~kg} \mathrm{U} / \text { day }}{2722 \mathrm{~kg} \mathrm{U} / \text { day }}=3.04 \times 10^{-4} \frac{\mathrm{kg} \mathrm{U}}{\mathrm{kg} \mathrm{U} \text { (input) }}
\end{aligned}
$$

3-3. Thorium-230 (38, p. 5-13)

a

$1.63 \frac{\mu \mathrm{Ci}}{\text { day }} \times \frac{49.6 \mathrm{~g} \mathrm{Th}-230}{\mathrm{C} i \mathrm{Th}-230} \times \frac{\mathrm{Ci}}{10^{6} \mu \mathrm{Ci}} \times \frac{\mathrm{kg}}{10^{3} \mathrm{~g}}=8.08 \times 10^{-8} \frac{\mathrm{kg}}{\text { day }}$

$\frac{8.08 \times 10^{-8} \frac{\mathrm{kg}}{\mathrm{day}}}{2722 \frac{\mathrm{kg} \mathrm{U}}{\text { day }}}=2.97 \times 10^{-11} \frac{\mathrm{kg} \mathrm{Th}-230}{\mathrm{~kg} \mathrm{U}}$ 
REFERENCE CALCULATIONS: Milling Facility (cont'd)

3-4. (7, p. 97) (6, p. 31)

$\frac{80 \mathrm{gal}}{\min } \times \frac{1440 \mathrm{~min}}{\text { day }} \times \frac{3785 \mathrm{ml}}{\mathrm{gal}} \times 2.2 \times 10^{-5} \frac{\mu \mathrm{Ci}}{\mathrm{ml}} \times \frac{\mathrm{Ci}}{10^{6} \mathrm{Ci}^{2}} \times \frac{\mathrm{g}^{230} \mathrm{Th}}{1.95 \times 10^{-2} \mathrm{Ci}} \times \frac{\mathrm{kg}}{10^{3} \mathrm{~g}}$

$4.92 \times 10^{-4} \frac{\mathrm{kg}^{230} \mathrm{Th}}{\text { day }} \quad \therefore \frac{4.92 \times 10^{-4} \mathrm{~kg}^{230} \mathrm{Th} / \text { day }}{2722 \mathrm{~kg} \mathrm{U} / \text { day }}=1.81 \times 10^{-7} \frac{\mathrm{kg}^{230} \mathrm{Th}}{\mathrm{kg} \mathrm{U} \text { (input) }}$

3-5. Radium-226 (38, p. 5-12)

Dִ

$1.45 \frac{\mu \mathrm{Ci}}{\text { day }} \times \frac{1.01 \mathrm{~g} \mathrm{Ra}-226}{\mathrm{C} i \mathrm{Ra}-226} \times \frac{\mathrm{Ci}}{10^{6} \mu \mathrm{Ci}} \times \frac{\mathrm{kg}}{10^{3} \mathrm{~g}}=1.46 \times 10^{-9} \frac{\mathrm{kg}}{\text { day }}$

$\frac{1.46 \times 10^{-9} \frac{\mathrm{kg}}{\mathrm{day}}}{2722 \frac{\mathrm{kg} \mathrm{U}}{\mathrm{day}}} 5.38 \times 10^{-13} \frac{\mathrm{kg} \mathrm{Ra}-226}{\mathrm{~kg} \mathrm{U}}$ 
REFERENCE CALCULATIONS: Milling Facility (cont'd)

3-6. $80 \frac{\mathrm{gal}}{\mathrm{min}} \times \frac{1440 \mathrm{~min}}{\mathrm{day}} \times 3785 \frac{\mathrm{ml}}{\mathrm{gal}} \times 3.5 \times 10^{-7} \frac{\mu \mathrm{Cl}}{\mathrm{ml}} \times \frac{\mathrm{Ci}}{10^{6}{ }_{\mu \mathrm{Ci}}} \times \frac{\mathrm{g}^{226} \mathrm{Ra}}{0.99 \mathrm{Ci}} \times \frac{\mathrm{kg}}{10^{3} \mathrm{~g}}=$
$1.54 \times 10^{-7} \frac{\mathrm{kg}{ }_{\mathrm{day}}^{226}}{\mathrm{Ra}} \quad \therefore \frac{1.54 \times 10^{-7} \mathrm{~kg} 226_{\mathrm{Ra} / \mathrm{day}}}{2722 \mathrm{~kg} \mathrm{U} / \mathrm{day}}=5.66 \times 10^{-11} \frac{\mathrm{kg}{ }^{226_{\mathrm{Ra}}}}{\mathrm{kg} \mathrm{U} \text { (input) }}$

4-19-77

3-7. Radon-222 (38, p. 5-14)

$5.46 \times 10^{5} \frac{\mu C i}{d a y} \times \frac{6.46 \times 10^{-6} \mathrm{~g}}{C i} \times \frac{C i}{10^{6} \mu C i} \times \frac{\mathrm{kg}}{10^{3} \mathrm{~g}}=3.53 \times 10^{-9}$

$\mathrm{RF}=\frac{3.53 \times 10^{-9} \mathrm{~kg} \mathrm{Rn} 222}{2722 \mathrm{~kg} \mathrm{U}}=1.3 \times 10^{-12} \frac{\mathrm{kg} \mathrm{Rn} 222}{\mathrm{~kg} \mathrm{U}}$ 
REFERENCE CALCULATIONS: Milling Facility (cont'd)

3-8 Rock dust (39)

Particulate emissions; 3-year average (1975-77)

Crusher

Fine Orebin

Leach

Sample Room

Yellowcake Dryer

TOTAL

$$
7.1 \times 10^{3} \text { grams/day }
$$$$
1.5 \times 10^{2}
$$$$
6.9 \times 10^{1}
$$$$
2.7 \times 10^{2}
$$$$
7.6 \times 10^{2}
$$

$8.4 \times 10^{3}$ grams/day

$\underset{0}{i}$

$$
\begin{aligned}
& 8.4 \times 10^{3} \frac{\text { grams }}{\text { day }} \times \frac{\mathrm{kg}}{10^{3} \mathrm{gr}}=8.4 \mathrm{~kg} / \text { day } \\
& \mathrm{RF}=\frac{8.4}{2722} \frac{\mathrm{kg} \text { particulate }}{\mathrm{kg} \mathrm{U}}=3.09 \times 10^{-3} \underbrace{\mathrm{kg} \mathrm{U}}_{\mathrm{kg} \text { particulate }}
\end{aligned}
$$

3-9. $[285$ (leaching) +175 (boilers) $] \frac{1 \mathrm{bs} \mathrm{SO}_{2}}{\mathrm{yr}} \times \frac{.4536 \mathrm{~kg}}{1 \mathrm{~b}} \times \frac{\mathrm{yr}}{365 \text { days }}=5.72 \times 10^{-1} \mathrm{~kg} \mathrm{\textrm {SO } _ { 2 }}$

$$
\begin{aligned}
& 5.72 \times 10^{-1} \mathrm{~kg} \mathrm{SO} / \text { day } 2.10 \times 10^{-4} \mathrm{~kg} \mathrm{SO} \mathrm{SO}_{2} \\
& 2722 \mathrm{~kg} \mathrm{U/day}=\mathrm{kgU} \text { (input) }
\end{aligned}
$$


REFERENCE CALCULATIONS: Milling Facility (cont'd)

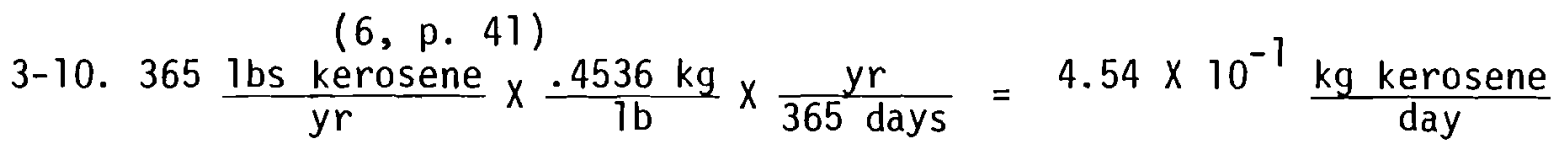

$\therefore \frac{4.54 \times 10^{-1} \mathrm{~kg} \text { kerosene } / \text { day }}{2722 \mathrm{~kg} \mathrm{U} / \text { day }}=1.67 \times 10^{-4} \frac{\mathrm{kg} \text { kerosene }}{\mathrm{kg} \mathrm{U} \text { (input) }}$

(6, p. 41)

3-11. $1750 \frac{\text { lbs particulate (from boilers) }}{\mathrm{yr}} \times \frac{.4536 \mathrm{~kg}}{1 \mathrm{~b}} \times \frac{\mathrm{yr}}{365 \text { days }}=2.17 \frac{\mathrm{kg} \text { particulate }}{\text { day }}$

$\therefore \frac{2.17 \mathrm{~kg} \text { particulate } / \text { day }}{2722 \mathrm{~kg} \mathrm{U} / \text { day }}=7.97 \times 10^{-4} \frac{\mathrm{kg} \text { particulate }}{\mathrm{kg} \mathrm{U} \text { (input) }}$

(6, p. 41)

3-12. $35,000 \frac{1 \mathrm{bs} \mathrm{NO} x}{\mathrm{yr}} \times \frac{.4536 \mathrm{~kg}}{1 \mathrm{~b}} \times \frac{\mathrm{yr}}{365 \text { days }}=4.35 \times 10^{1} \frac{\mathrm{kg} \mathrm{NO} \mathrm{x}}{\text { day }}$

$\therefore \frac{4.35 \times 10^{7} \mathrm{~kg} \mathrm{N0} / \mathrm{day}}{2722 \mathrm{~kg} \mathrm{U/day}}=1.60 \times 10^{-2} \mathrm{~kg} \mathrm{N0} \frac{\mathrm{kg} \mathrm{U} \text { (input) }}{\mathrm{kg}}$

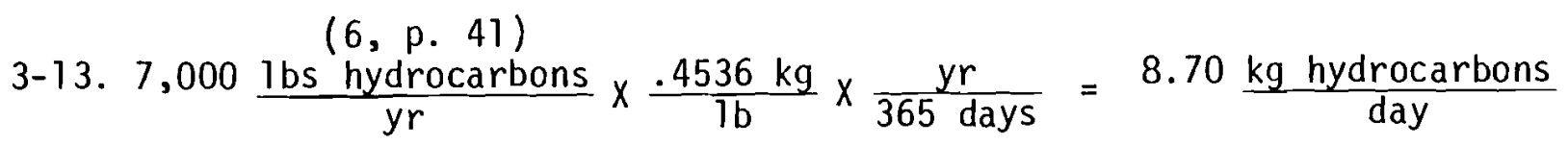


$\therefore \frac{8.70 \mathrm{~kg} \text { hydrocarbons } / \text { day }}{2722 \mathrm{~kg} / \mathrm{V} / \mathrm{day}}=3.20 \times 10^{-3} \frac{\mathrm{kg} \text { hydrocarbons }}{\mathrm{kg} \mathrm{U} \text { (input) }}$

3-14. $10,500 \frac{\mathrm{lbs} \text { organic acids }}{\mathrm{yr}} \times \frac{.4536 \mathrm{~kg}}{1 \mathrm{~b}} \times \frac{\mathrm{yr}}{365 \text { days }}=1.30 \times 10^{7} \frac{\mathrm{kg} \text { org. acids }}{\text { day }}$

$\therefore \quad \frac{1.30 \times 10^{7} \mathrm{~kg} \text { organic acids } / \text { day }}{2722 \mathrm{~kg} \mathrm{U} / \text { day }}=4.78 \times 10^{-3} \frac{\mathrm{kg} \text { organic acids }}{\mathrm{kg} \mathrm{U} \text { (input) }}$

3-15. $1752 \frac{\text { lbs aldehydes }}{\mathrm{yr}} \times \frac{.4536 \mathrm{~kg}}{1 \mathrm{~b}} \times \frac{\mathrm{yr}}{365 \text { days }}=2.18 \mathrm{~kg}$ aldehydes

$\therefore \frac{2.18 \mathrm{~kg} \mathrm{aldehydes} / \text { day }}{2722 \mathrm{~kg} \mathrm{U} / \text { day }}=8.01 \times 10^{-4} \frac{\mathrm{kg} \text { aldehydes }}{\mathrm{kg} \mathrm{U} \text { (input) }}$

(8, p. 173) (7, p. 97)

3-16. $0.5 \frac{\mathrm{g} \mathrm{Ca}}{1 \text { iter }} \times 3.785 \frac{1 \mathrm{iter}}{\mathrm{gal}} \times \frac{80 \mathrm{gal}}{\mathrm{min}} \times \frac{1440 \mathrm{~min}}{\text { day }} \times \frac{\mathrm{kg}}{10^{3} \mathrm{~g}}=2.18 \times 10^{2} \mathrm{~kg} \mathrm{Ca}$

$\therefore \frac{2.18 \times 10^{2} \mathrm{~kg} \mathrm{Ca} / \text { day }}{2722 \mathrm{~kg} \mathrm{U} / \mathrm{day}}=8.01 \times 10^{-2} \frac{\mathrm{kg} \mathrm{Ca}}{\mathrm{kg} \mathrm{U} \text { (input) }}$

3-17. $\quad \frac{(8, \mathrm{~g} .173)}{1 \text { fiter }} \times \frac{3.785 \text { liter }}{\text { gal }} \times \frac{(70 \mathrm{gal}}{\mathrm{min}} \times \frac{1440 \mathrm{~min}}{\text { day }} \times \frac{\mathrm{kg}}{10^{3} \mathrm{~g}}=4.36 \times 10^{2} \mathrm{~kg} \mathrm{Fe}$ 
REFERENCE CALCULATIONS: Milling Facility (cont'd)

3-22. $5 \times 10^{-3} \frac{\mathrm{g} \mathrm{F}}{1 \mathrm{iter}} \times \frac{3.785 \mathrm{liter}}{\mathrm{gal}} \times \frac{(70 \mathrm{gal}}{\min } \times \frac{1440 \mathrm{~min}}{\text { day }} \times \frac{\mathrm{kg}}{10^{3} \mathrm{~g}}=2.18 \frac{\mathrm{kg} \mathrm{F}}{\text { day }}$

$\therefore \frac{2.18 \mathrm{~kg} \mathrm{~F} / \text { day }}{2722 \mathrm{~kg} \mathrm{U} / \mathrm{day}}=8.01 \times 10^{-4} \frac{\mathrm{kg} \mathrm{F}}{\mathrm{kg} \mathrm{U} \text { (input) }}$

(8, p. 173)
3-23. $1 \times 10^{-4} \frac{\mathrm{g} \mathrm{V}}{1 \mathrm{ter}} \times \frac{3.7851 \mathrm{iter}}{\mathrm{gal}} \times \frac{(7, \mathrm{gal}}{\min } \times \frac{97)}{\text { day }} \times \frac{\mathrm{kg}}{10^{3} \mathrm{~g}}=4.36 \times 10^{-2} \mathrm{~kg} \mathrm{~V}$
day

$\therefore \frac{4.36 \times 10^{-2} \mathrm{kgV} / \text { day }}{2722 \mathrm{~kg} \mathrm{U/day}}=1.60 \times 10^{-5} \frac{\mathrm{kg} \mathrm{V}}{\mathrm{kg} \mathrm{U} \text { (input) }}$

(8, p. 173)

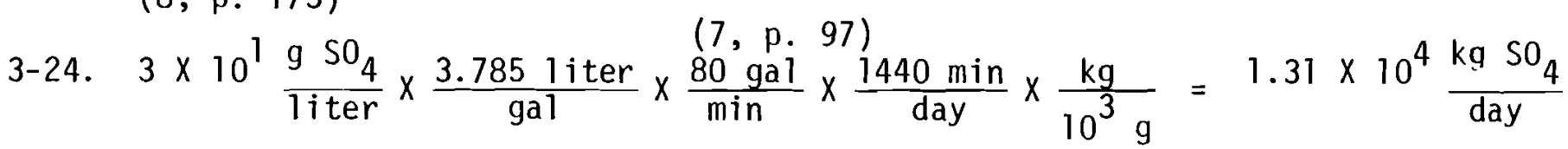

$\therefore \frac{1.31 \times 10^{4} \mathrm{~kg} \mathrm{S0} / \text { day }}{2722 \mathrm{~kg} \mathrm{U} / \text { day }}=4.81 \frac{\mathrm{kg} \mathrm{SO}_{4}}{\mathrm{~kg} \mathrm{U} \text { (input) }}$

$\therefore \quad \frac{1.31 \times 10^{2} \mathrm{~kg} \mathrm{Cl} / \text { day }}{2722 \mathrm{~kg} \mathrm{U} / \mathrm{day}}=4.81 \times 10^{-2} \frac{\mathrm{kg} \mathrm{Cl}}{\mathrm{kg} \mathrm{U} \text { (input) }}$

(8, p. 173)
$3-25.3 \times 10^{-1} \frac{\mathrm{g} \mathrm{Cl}}{1 \mathrm{iter}} \times \frac{3.785 \mathrm{liter}}{\mathrm{gal}} \times \frac{(70 \mathrm{gal}}{\min } \times \frac{97)}{\text { day }} \times \frac{\mathrm{kg}}{10^{3} \mathrm{~g}}=1.31 \times 10^{2} \mathrm{~kg} \mathrm{Cl}$
day 
REFERENCE CALCULATIONS: Milling Facility (cont'd)

(from ref. calc. 3-1) $\quad(4, \mathrm{p} . \mathrm{B}-35)$
$3-26.2 .48 \times 10^{-4} \frac{\mathrm{kg} \mathrm{U}}{\mathrm{kg} \mathrm{U} \text { (input) }} \times 99.2830 \%{ }^{238 U} \mathrm{in} \mathrm{nat'1} \mathrm{U}=2.46 \times 10^{-4} \frac{\mathrm{kg}^{238} \mathrm{U}}{\mathrm{kg} U \text { (input) }}$

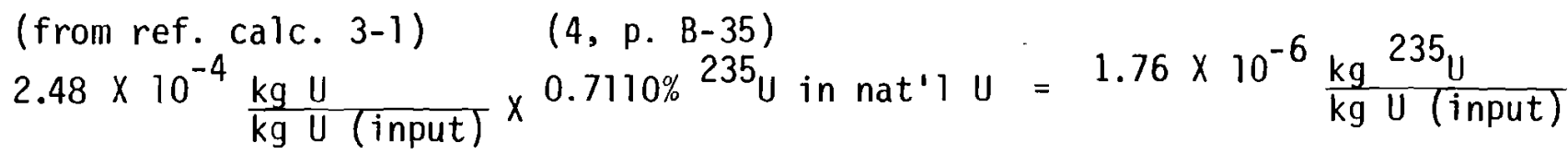

(from ref. calc. 3-1) (4, p. B-35)

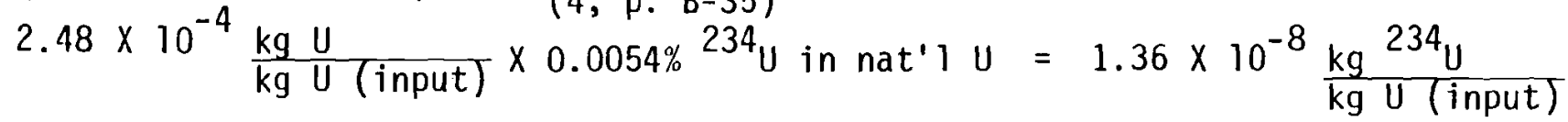

(from ref. calc. 3-2) (4, p. B-35)

$\underset{\perp}{\omega} \quad 3-27.3 .04 \times 10^{-4} \frac{\mathrm{kg} \mathrm{U}}{\mathrm{kg} \mathrm{U} \text { (input) }} \times 99.2830 \%{ }^{238} \mathrm{U}$ in nat'1 $\mathrm{U}=3.02 \times 10^{-4} \frac{\mathrm{kg}}{\mathrm{kg} U \text { (input) }}$

(from ref. calc. 3-2) (4, p. B-35)

$3.04 \times 10^{-4} \frac{\mathrm{kg} \mathrm{U}}{\mathrm{kgU} \text { (input) }} \times 0.7110 \%{ }^{235} \mathrm{U}$ in nat'1 $U=2.15 \times 10^{-6} \frac{\mathrm{kg}{ }^{235} \mathrm{U}}{\mathrm{kg} U \text { (input) }}$

(from ref. calc. 3-2)

$3.04 \times 10^{-4} \mathrm{kgu} \quad(4, \mathrm{p} . \mathrm{B}-35)$ $\frac{\mathrm{kg} U \text { (input) }}{\mathrm{kg}} \times 0.0054 \%{ }^{234} \mathrm{U}$ in nat'1 $\mathrm{U}=1.64 \times 10^{-8} \frac{\mathrm{kg}{ }^{234} \mathrm{U}}{\mathrm{kg} U \text { (input) }}$

(6, p. 41)

3-28. $3500 \frac{\text { lbs } \mathrm{NH}_{3}}{\mathrm{yr}}$ (from boilers) $\times \frac{.4536 \mathrm{~kg}}{1 \mathrm{~b}} \times \frac{\mathrm{yr}}{365 \text { days }}=4.35 \frac{\mathrm{kg} \mathrm{NH_{3 }}}{\text { day }}$

$\therefore \frac{4.35 \mathrm{kgNH}_{3} / \text { day }}{2722 \mathrm{~kg} \mathrm{U/day}}=1.60 \times 10^{-3} \frac{\mathrm{kg} \mathrm{NH}_{3} \text { (from boilers) }}{\mathrm{kgU} \text { (input) }}$ 


\begin{tabular}{|c|c|c|c|c|c|c|}
\hline \multirow[b]{3}{*}{ Material Released } & \multicolumn{5}{|c|}{ CONVERSION FACILITY CHEMICAL RELEASE FACTOR $\mathrm{Kg} / \mathrm{Kg} \cup$ (INPUT) } & \multirow[t]{2}{*}{$F-4-21-77$} \\
\hline & \multicolumn{3}{|c|}{ Air Borne } & \multicolumn{2}{|r|}{ Liquid } & \\
\hline & Factor & Reliability & $\begin{array}{l}\text { Ref. } \\
\text { Calc. }\end{array}$ & Factor & Reliability & $\begin{array}{l}\text { Ref. } \\
\text { Calc. }\end{array}$ \\
\hline Uranium (natural) & $4 \times 10^{-6}$ & 2 & $4-1$ & $1 \times 10^{-3}$ & 2 & $4-8$ \\
\hline Thor ium - 230 & & & & & & \\
\hline Radium - 226 & & & & & & \\
\hline Nitrogen $0 x$ ides $\left(N O_{x}\right)$ & $2 \times 10^{-2}$ & 2 & $4-2$ & & & \\
\hline Nitric Acid $\left(\mathrm{HNO}_{3}\right)$ & & & & & & \\
\hline Hydrogen Fluor ide (HF) & $3 \times 10^{-4}$ & 2 & $4-3$ & & & \\
\hline Fluor ine $\left(F_{2}\right)$ & $1 \times 10^{-5}$ & 2 & $4-4$ & & & \\
\hline Hydrogen $\left(\mathrm{H}_{2}\right)$ & & & & & & \\
\hline Hexane & $1.7 \times 10^{-2}$ & 3 & $4-5$ & & & \\
\hline $\begin{array}{l}\text { Sulfur Oxides }\left(\mathrm{SO}_{x}\right) \\
\text { Hydrogen Sulfide }\left(\mathrm{H}_{2} \mathrm{~S}\right)\end{array}$ & $5 \times 10^{-3}$ & 2 & $4-6$ & & & \\
\hline Nonradioact ive & & & & & & \\
\hline Particulate & $7 \times 10^{-4}$ & 2 & $4-7$ & & & \\
\hline Carbon Monox ide ( $\mathrm{CO})$ & $7 \times 10^{-4}$ & 2 & $4-7$ & & & \\
\hline Hydrocarbons & $1 \times 10^{-4}$ & 2 & $4-7$ & & & \\
\hline Nitrate $\left(\mathrm{NO}_{3}\right)$ & & & & $7 \times 10^{-4}$ & 2 & $4-8$ \\
\hline Fluoride $(F)$ & & & & $4 \times 10^{-4}$ & 2 & $4-8$ \\
\hline Calcium (Ca) & & & & $7 \times 10^{-3}$ & 2 & $4-8$ \\
\hline Sulfate $\left(\mathrm{SO}_{4}\right)$ & & & & $8 \times 10^{-3}$ & 2 & $4-8$ \\
\hline
\end{tabular}


CONVERSION FACILITY RADIONUCLIDE RELEASE FACTOR $\mathrm{Kg} / \mathrm{Kg} U$ (INPUT)

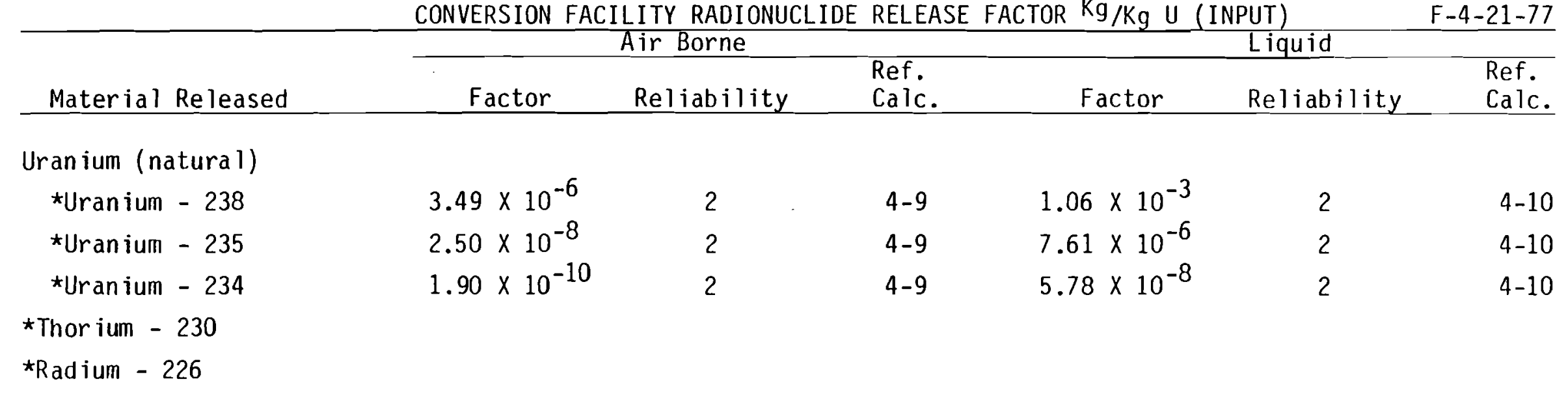

*Has Data Base In Computer Model

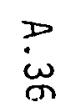


REFERENCE CALCULATIOIS: CONVERSION FACILITY (365 Operating Days/yr)

4-1. U input to the conversion facility:

$\frac{10,000 \text { short tons } U}{y r} \times \frac{907.2 \mathrm{~kg}}{\text { short ton }} \times \frac{\mathrm{yr}}{365 \text { days }}=2.485 \times 10^{4} \frac{\mathrm{kg} \mathrm{U}}{\text { day }}$

U airborne output from the conversion facility:

Sampled preparation room air exhaust and HF scrubber

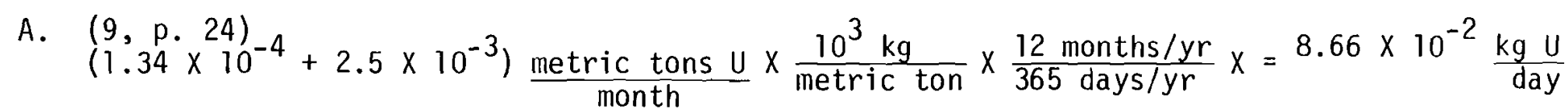

$\mathrm{UO}_{3}$ dust collector exhaust

B. $(10, p .61)$

$\left(2 \times 10^{-6} \frac{\text { metric tons } \mathrm{U}}{\text { month }} \times \frac{10^{3} \mathrm{~kg}}{\text { metric ton }} \times \frac{12 \text { months } / \mathrm{yr}}{365 \text { days } / \mathrm{yr}} \times \frac{4.33}{1}=2.85 \times 10^{-4} \mathrm{~kg} \mathrm{U}\right.$

(assumed linear scaleup

from 2312 s.t. $\mathrm{U} / \mathrm{yr} \rightarrow 10,000$ s.t. $\mathrm{U} / \mathrm{yr}$ )

$\stackrel{D}{\dot{w}}$

$$
\text { month } \times \frac{10}{\text { metric ton }} \times \frac{1265 \text { days/yr }}{365} \frac{4}{1}=2.85 \times 10-4 \text { day }
$$

Reduction off-gas to plant stack

C. (from ref.

(calc. 4-1)

$2.485 \times 10^{4} \frac{\mathrm{kg} \mathrm{U}}{\text { day }} X$

$\begin{array}{cc}\text { (assumed amt. released } & \text { (assumed SMF } \\ \text { to eff. control) } & 80 \% \text { eff.) }\end{array}$

$.001 \%$

$\times \quad 20 \%$
(assumed bag filter $99 \%$ eff.)
$x \quad 1 \%$

$=4.97 \times 10^{-4} \frac{\mathrm{kg} \mathrm{U}}{\text { day }}$

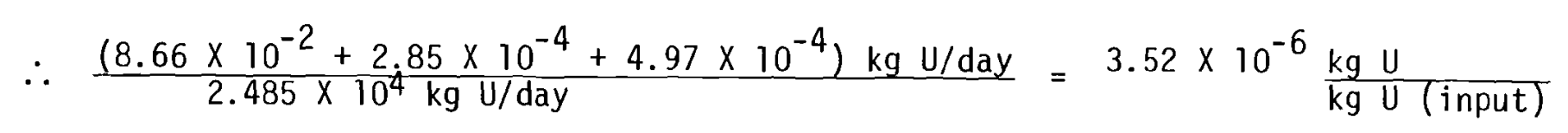

4-2. Assuming 5.8 metric tons $N 0$ /month (11, p. III-8) for burning 65 million cubic feet of natural gas per month in stoichiometric air (9, p. 24) then: 
REFERENCE CALCULATIONS: Conversion Facility (cont'd)

(see above) $(9$, p. 24)

$[5.8+12.1$ (from absorber tail gas) $] \frac{\text { metric tons }{ }^{N 0} \times}{\text { month }} \times \frac{12 \text { months }}{365 \text { days }} \times \frac{10^{3} \mathrm{~kg}}{\text { metric ton }}=5.88 \times 10^{2} \mathrm{~kg} \mathrm{NO}_{\mathrm{x}}$

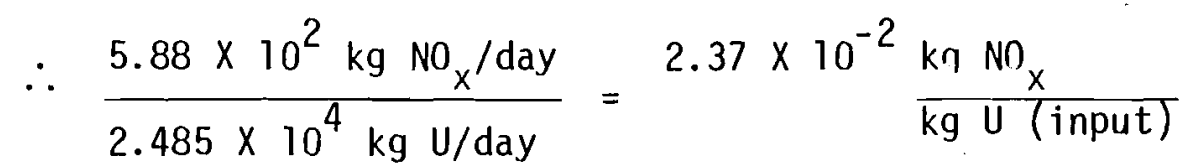

\section{4-3. (from ref. calc. 4-1)}

(amount of orig. $\mathrm{U}$ as $\mathrm{UF}_{4}$ assumed to be on exit filters)

A. $2.485 \times 10^{4} \frac{\mathrm{kg} \mathrm{U}}{\text { day }} \times \frac{314 \mathrm{~kg} \mathrm{UF}}{238 \mathrm{~kg} \mathrm{U}} \times$

$$
5 \times 10^{-4} \times \frac{80 \mathrm{~kg} \mathrm{HF}}{374 \mathrm{~kg} \mathrm{U}} \times
$$

(assuming $\mathrm{NaOH}$ scrubbing is $95 \%$ efficient) .05 $=2.09 \times 10^{-1} \frac{\mathrm{kg} \mathrm{HF}}{\text { day }}$ (from miscellaneous digester and associated effluent treatment)

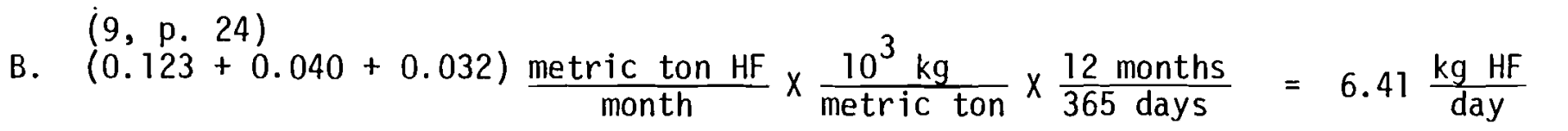
(from HF scrubber, $F_{2}$
emergency vent, $F_{2}$ rework )

$\therefore \frac{\left(2.09 \times 10^{-1}+6.41\right) \mathrm{kg} \mathrm{HF} / \text { day }}{2.485 \times 10^{4} \mathrm{~kg} \mathrm{U} / \text { day }}=2.66 \times 10^{-4} \frac{\mathrm{kg} \mathrm{HF}}{\mathrm{kg} \mathrm{U} \text { (input) }}$ 


\section{REFERENCE CALCULATIONS: Conversion Facility (cont'd)}

4-4. $\frac{\left(9,011 \text { metric ton } \mathrm{F}_{2}\right.}{\text { month }} \times \frac{10^{3} \mathrm{~kg}}{\text { metric ton }} \times \frac{12 \text { months }}{365 \text { days }}=3.62 \times 10^{-1} \frac{\mathrm{kg} \mathrm{F}}{\frac{24}{\text { day }}}$

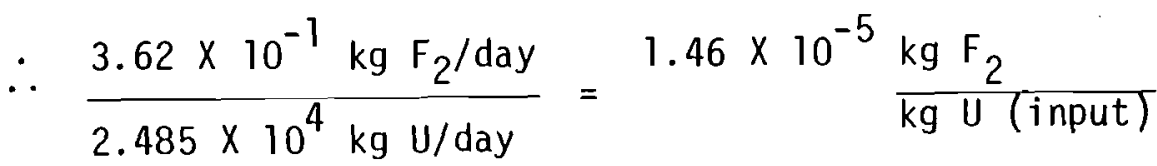

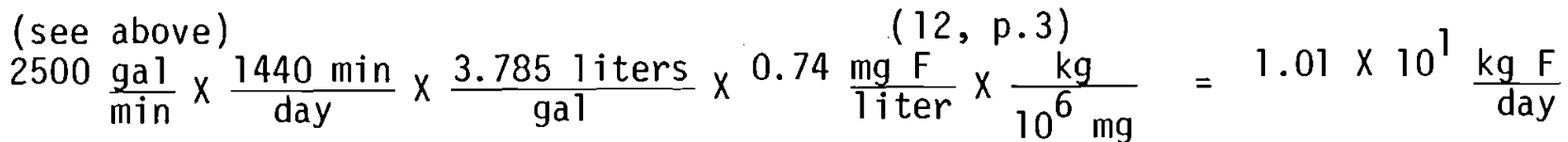

$\dot{w}_{i}$

$\therefore \frac{1.01 \times 10^{1} \mathrm{~kg} \mathrm{~F} / \text { day }}{2.485 \times 10^{4} \mathrm{~kg} \mathrm{U} / \text { day }}=4.06 \times 10^{-4} \frac{\mathrm{kgF}}{\mathrm{kg} \mathrm{U} \text { (input) }}$

(see above) (12, p.3)

$2500 \frac{\mathrm{gal}}{\mathrm{min}} \times \frac{1440 \mathrm{~min}}{\text { day }} \times \frac{3.785 \mathrm{liter}}{\text { gal }} \times 12.13 \frac{\mathrm{mgCa}}{1 \mathrm{iter}} \times \frac{\mathrm{kg}}{10^{6} \mathrm{mg}}=1.65 \times 10^{2} \mathrm{~kg} \mathrm{Ca}$

$\therefore \frac{1.65 \times 10^{2} \mathrm{~kg} \mathrm{Ca} / \text { day }}{2.485 \times 10^{4} \mathrm{~kg} \mathrm{U} / \text { day }}=6.65 \times 10^{-3} \frac{\mathrm{kg} \mathrm{Ca}}{\mathrm{kg} \mathrm{U(input)}}$

4-5. Hexane Release Factor

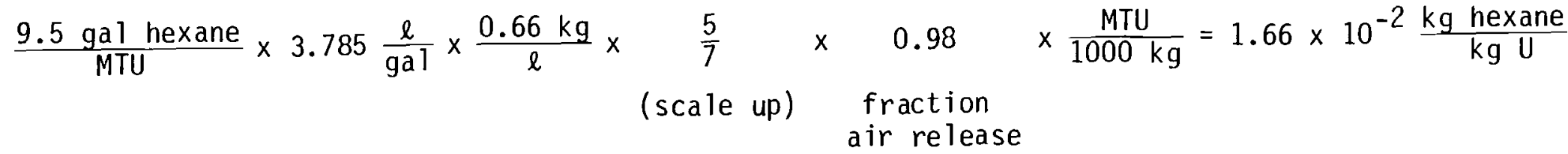


4-6. Assuming 651 bs $\mathrm{SO}_{x} /$ month $(8, \mathrm{p} . \mathrm{V}-10)$ for burning 65 million cubic feet of natural gas per month in stoichiometric air $(9$, p. 24$)$ then:

$$
\begin{aligned}
& {\left[\left(\frac{65 \text { 1bs S0 } x}{\text { month }} \times \frac{.4536 \mathrm{~kg}}{1 \mathrm{~b}}\right)+\left(\frac{4.10 \text { metric tons } \mathrm{S} 0 \mathrm{x}}{\text { month }} \times \frac{10^{3} \mathrm{~kg}}{\text { metric ton }}\right)\right] \times \frac{12 \text { months }}{365 \text { days }}=1.36 \times 10^{2} \mathrm{~kg} \mathrm{S0} \times \frac{19}{\text { day }}} \\
& \therefore \frac{1.36 \times 10^{2} \mathrm{~kg} \mathrm{S0} / \text { day }}{2.485 \times 10^{4} \mathrm{~kg} \mathrm{U} / \text { day }}=5.46 \times 10^{-3} \frac{\mathrm{kg} \mathrm{S}{ }_{x}}{\mathrm{~kg} \mathrm{U} \text { (input) }}
\end{aligned}
$$

4-7. Basis for the following numbers is the use of 65 million cubic feet of natural gas per month and EPA emission factors $(1, \mathrm{p} .1 .4-2)$.

$$
\begin{aligned}
& \therefore \frac{18 \mathrm{~kg} \mathrm{particulate/day}}{2.485 \times 10^{4} \mathrm{~kg} \mathrm{U} / \text { day }}=7.24 \times 10^{-4} \frac{\mathrm{kg} \text { particulate }}{\mathrm{kg} \mathrm{U} \text { (input) }} \\
& \therefore \frac{17 \mathrm{~kg} \mathrm{C0} / \text { day }}{2.485 \times 10^{4} \mathrm{~kg} \mathrm{U} / \text { day }}=6.84 \times 10^{-4} \frac{\mathrm{kg} \mathrm{C}}{\mathrm{kg} \mathrm{U} \text { (input) }} \\
& \therefore \frac{3 \mathrm{~kg} \mathrm{hydrocarbons/ \text {day }}}{2.485 \times 10^{4} \mathrm{~kg} \mathrm{U} / \text { day }}=1.21 \times 10^{-4} \frac{\mathrm{kg} \mathrm{hydrocarbons}}{\mathrm{kg} \mathrm{U(input)}}
\end{aligned}
$$

4-8. Assuming the liquid effluents leave the facility in a $2,500 \mathrm{gal} / \mathrm{min}$ stream (12, p.3) and included the concentrations of chemicals given in (12, p. 3$)$ then:

$$
\begin{aligned}
& \text { (see above) } \\
& 2500 \frac{\text { gal }}{\min } \times \frac{1440 \mathrm{~min}}{\text { day }} \times \frac{3.7851 \text { iter }}{\text { gal }} \times 1.95 \frac{\mathrm{mg} \mathrm{U}}{1 \mathrm{iter}} \times \frac{\mathrm{kg}}{10^{6} \mathrm{mg}}=2.66 \times 10^{1} \frac{\mathrm{kg} \mathrm{U}}{\text { day }}
\end{aligned}
$$




\section{REFERENCE CALCULATIONS: Conversion Facility (cont'd)}

$\therefore \frac{2.66 \times 10^{1} \mathrm{~kg} \mathrm{U} / \text { day }}{2.485 \times 10^{4} \mathrm{~kg} \mathrm{U} / \text { day }}=1.07 \times 10^{-3} \mathrm{kgU \text {(in } 7 \text { iquid effluent) }}$

$$
\begin{aligned}
& \text { (see above) } \\
& 2500 \frac{\mathrm{gal}}{\min } \times \frac{1440 \mathrm{~min}}{\mathrm{day}} \times \frac{3.785 \mathrm{liter}}{\mathrm{gal}} \times \frac{1.31 \mathrm{mg} \mathrm{NO}}{1 \mathrm{iter}} \times \frac{\mathrm{kg}}{10^{6} \mathrm{mg}}=1.79 \times 10^{1} \frac{\mathrm{kg} \mathrm{NO}_{3}}{\text { day }} \\
& \therefore \frac{1.79 \times 10^{1} \mathrm{~kg} \mathrm{NO}_{3} / \text { day }}{2.485 \times 10^{4} \mathrm{~kg} \mathrm{U} / \text { day }}=7.18 \times 10^{-4} \frac{\mathrm{kg} \mathrm{NO}_{3}}{\mathrm{~kg} \mathrm{U} \text { (input) }}
\end{aligned}
$$

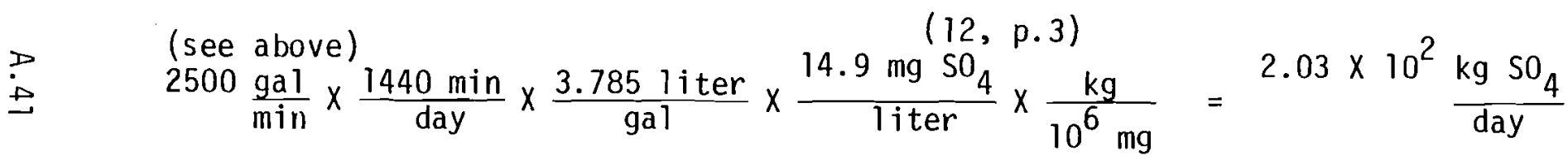

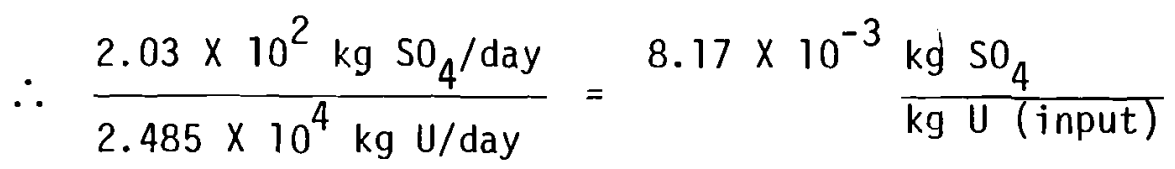

$4-21-77$

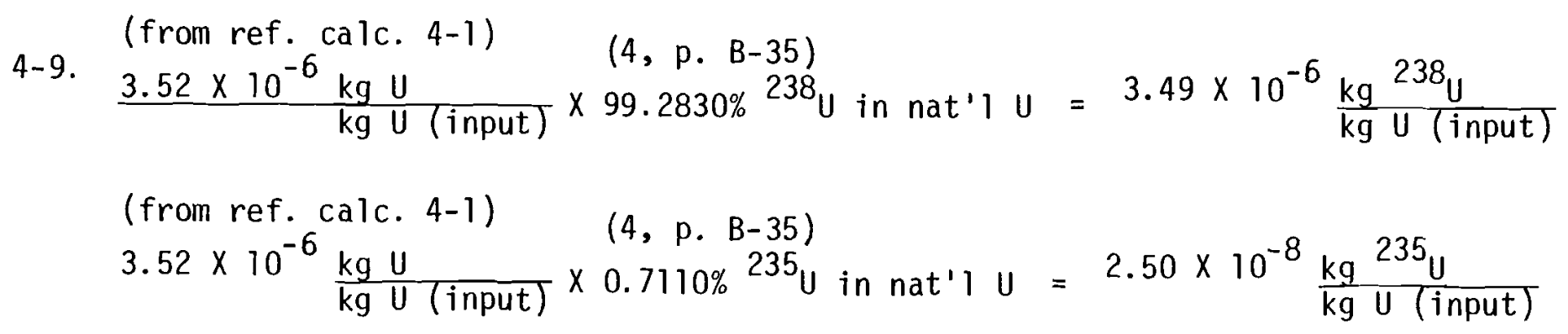


REFERENCE CALCULATIONS: Conversion Facility (cont'd)

$\begin{array}{ll}\text { (from ref. calc. 4-1) } & (4, \mathrm{p} . \mathrm{B}-35) \\ \frac{3.52 \times 10^{-6} \mathrm{~kg} \mathrm{U}}{\mathrm{kg} U \text { (input) }} \times 0.0054 \%{ }^{234} \mathrm{U} \text { in nat'l } \mathrm{U}=1.90 \times 10^{-10} \frac{\mathrm{kg}}{\mathrm{kg} U \text { (input) }}\end{array}$

(from ref. calc. 4-8)

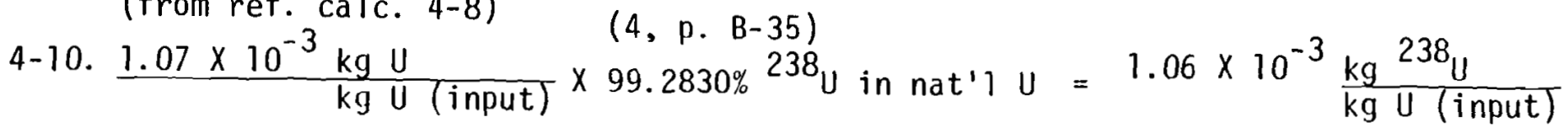

(from ref. calc, 4-8)

$\frac{1.07 \times 10^{-3} \mathrm{~kg} U}{\mathrm{~kg} U \text { (input) }} \times \frac{(4, \mathrm{p} .8-35)}{0.7110 \%}{ }^{235} U$ in nat' $1 U=7.61 \times 10^{-6} \frac{\mathrm{kg}{ }^{235} \mathrm{U}}{\mathrm{kg} U \text { (input) }}$

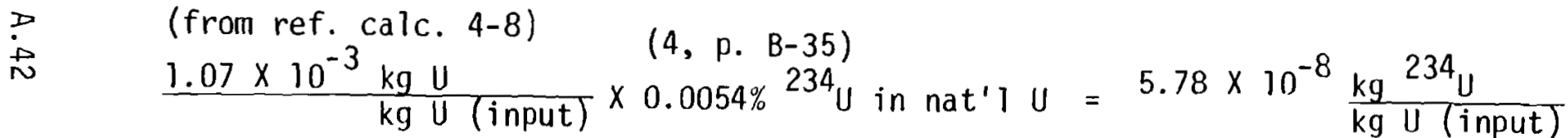




\begin{tabular}{|c|c|c|c|c|c|c|}
\hline \multirow{2}{*}{ Material Re leased } & \multicolumn{3}{|c|}{ Air Borne } & \multicolumn{3}{|c|}{ Liquid } \\
\hline & Factor & Reliability & $\begin{array}{l}\text { Ref. } \\
\text { Calc. }\end{array}$ & Factor & Reliability & $\begin{array}{l}\text { Ref. } \\
\text { Calc. }\end{array}$ \\
\hline Uranium & $1 \times 10^{-5}$ & 1 & $5-1$ & $7 \times 10^{-5}$ & 1 & $5-2$ \\
\hline Thor ium - 234 & & & & $1 \times 10^{-13}$ & 1 & $5-3$ \\
\hline Hydrogen Fluoride (HF) & $1 \times 10^{-3}$ & 1 & $5-4$ & & & \\
\hline Freon & $6 \times 10^{-3}$ & 1 & $5-5$ & & & \\
\hline Ammonia $\left(\mathrm{NH}_{3}\right)$ & $2 \times 10^{-4}$ & 1 & $5-6$ & & & \\
\hline Nitrogen $0 x$ ides $\left(\mathrm{NO}_{\mathrm{x}}\right)$ & $3 \times 10^{-1}$ & 1 & $5-7$ & & & \\
\hline Nonradioact ive & & & & & & \\
\hline Particulate & $2 \times 10^{-2}$ & $\because 1$ & $5-8$ & & & \\
\hline Sulfur Oxides $\left(\mathrm{SO}_{\mathrm{x}}\right)$ & $3 \times 10^{-1}$ & 1 & $5-9$ & & & \\
\hline Carbon Monoxide $(\mathrm{CO})$ & $4 \times 10^{-2}$ & 1 & $5-10$ & & & \\
\hline Hydrocarbons & $7 \times 10^{-3}$ & 1 & $5-11$ & & & \\
\hline Ozone $\left(\mathrm{O}_{3}\right)$ & $2 \times 10^{-5}$ & 1 & $5-12$ & & & \\
\hline Chlorothene & $1 \times 10^{-3}$ & 1 & $5-13$ & & & \\
\hline Trichlore thy lene & $2 \times 10^{-2}$ & 1 & $5-14$ & & & \\
\hline Acetone & $6 \times 10^{-4}$ & 1 & $5-15$ & & & \\
\hline Xylol (xylene) & $8 \times 10^{-5}$ & 1 & $5-16$ & & & \\
\hline Kerosene & $5 \times 10^{-4}$ & 1 & $5-17$ & & & \\
\hline Fluorine $\left(F_{2}\right)$ & & & & & & \\
\hline $\mathrm{Ch}$ lorine $\left(\mathrm{Cl}_{2}\right)$ & & & & $9 \times 10^{-4}$ & 1 & $5-24$ \\
\hline Nitrate $\left.\left(\mathrm{NO}_{3}\right)\right)$ & & & & $2 \times 10^{-2}$ & 1 & $5-18$ \\
\hline
\end{tabular}


Calc.

Factor

Reliability

Ref.

Aluminum (AI)

Floride (F)

Tributyl Phosphate

Varsol

Phosphate $\left(\mathrm{PO}_{4}\right)$

$1 \times 10^{-4}$

$2 \times 10^{-4}$

$3 \times 10^{-5}$

$9 \times 10^{-5}$

$2 \times 10^{-3}$

1
Calc.

Chloride (Cl)

Chromium ( $\mathrm{Cr}$ )

Zinc $(Z n)$

$1 \times 10^{-4}$

$1 \times 10^{-3}$

$5-19$

$5-20$

$5-21$

$5-22$

$5-23$

Sulfate $\left(\mathrm{SO}_{4}\right)$

Sulfuric Acid $\left(\mathrm{H}_{2} \mathrm{SO}_{4}\right)$

Boric Acid $\left(\mathrm{H}_{3} \mathrm{BO}_{3}\right)$

Nickel Sulfate $\left(\mathrm{NiSO}_{4}\right)$

Nickel Chloride $\left(\mathrm{NiCl}_{2}\right)$

Helium ( $\mathrm{He}$ )

$\mathrm{x}$

Hydrogen $\left(\mathrm{H}_{2}\right)$

Nitrogen $\left(\mathrm{N}_{2}\right)$

Argon (Ar)

Calcium (CA)

$x$

Sodium ( $\mathrm{Na}$ ) 
DIFFUSION ENRICHMENT (cont'd) CHEMICAL RELEASE FACTOR Kg/Kg U (INPUT) F-9-20-78 Air Borne Liquid

Material Released

\section{Iron (Fe)}

Boron (B)

Nickel ( $\mathrm{Ni}$ )

Magnesium ( $\mathrm{Mg}$ )

Ref.

Factor

Reliability

Calc.

Factor

Reliability

Ref.

Mo lybdenum (Mo)

Silver $(A g)$

Manganese (Mn)

\section{$x$}

$x$

$x$

$x$

$x$

$x$

$x$

$x$ Indicates Most Likely Form of Effluent 
DIFFUSION ENRICHMENT RADIONUCLIDE RELEASE FACTOR $\mathrm{kg} / \mathrm{Kg} U$ (INPUT)

Air Borne

Liquid

\begin{tabular}{|c|c|c|c|c|c|c|}
\hline \multirow[b]{2}{*}{ Material Released } & \multicolumn{3}{|c|}{ Air Borne } & \multicolumn{3}{|c|}{ Liquid } \\
\hline & Factor & Reliability & $\begin{array}{l}\text { Ref. } \\
\text { Calc. }\end{array}$ & Factor & Reliability & $\begin{array}{l}\text { Ref. } \\
\text { Calc. }\end{array}$ \\
\hline \multicolumn{7}{|l|}{ Uranium } \\
\hline *Uranium - 238 & $9.22 \times 10^{-6}$ & 1 & $5-27$ & $6.47 \times 10^{-5}$ & 1 & $5-28$ \\
\hline *Uranium - 235 & $3.37 \times 10^{-7}$ & 1 & $5-27$ & $5.15 \times 10^{-7}$ & 1 & $5-28$ \\
\hline *Uranium - 234 & $1.52 \times 10^{-13}$ & 1 & $5-27$ & $4.33 \times 10^{-9}$ & 1 & $5-28$ \\
\hline Thor ium - 234 & & & & $1 \times 10^{-13}$ & & $5-3$ \\
\hline
\end{tabular}

*Has Data Base In Computer Model 


\section{REFERENCE CALCULATIONS: DIFFUSION ENRICHMENT FACILITY (365 Operating Days/yr)}

$4-28-77$

5-1. Enriched U $\left(3.2 \%{ }^{235} U\right)$ output from diffusion enrichment facility based on $0.25 \%$ tails and $8.75 \times 10^{6}$ SWU/year:

(13, p. 40 and p. 41)

$8.75 \times 10^{6} \frac{\mathrm{kg} \mathrm{SWU}}{\mathrm{yr}} \times \frac{\mathrm{kg} \mathrm{U} \text { product }}{4.2 \mathrm{~kg} \mathrm{SWU}} \times \frac{\mathrm{yr}}{365 \text { days }}=5707.76 \frac{\mathrm{kg} 3.2 \%{ }^{235} \mathrm{U} \text { product }}{\text { day }}$

U airborne output from the diffusion enrichment facility: (assume just natural $U$ input)

$$
\begin{aligned}
& \text { (14, p. 2.3-50 and (30,\#31) (14,p. 2.3-50 and 2.3-140) (30,\#28) } \\
& {\left[\left(6.4 \times 10^{-3} \frac{\mathrm{Ci}}{\mathrm{yr}} \times \frac{{ }^{238} \mathrm{U}}{3.33 \times 10^{-7} \mathrm{Ci}^{238} \mathrm{U}}\right)+\left(1.5 \times 10^{-3} \frac{\mathrm{Ci}^{235} \mathrm{U}}{\mathrm{yr}} \times \frac{\mathrm{g}^{235} \mathrm{U}}{2.14 \times 10^{-6} \mathrm{Cj}{ }^{235} \mathrm{U}}\right)+\right.} \\
& \text { (14, p. 2.3-50 and 2.3-140) }(30, \# 27) \\
& \left.\left(3.9 \times 10^{-2} \frac{\mathrm{Ci}^{234} \mathrm{U}}{\mathrm{yr}} \times \frac{\mathrm{g}^{234} \mathrm{U}}{6.1 \times 10^{-3} \mathrm{Ci} 234 \mathrm{U}}\right)\right] \times \frac{\mathrm{yr}}{365 \text { days }} \times \frac{\mathrm{kg}}{10^{3} \mathrm{~g}}=5.46 \times 10^{-2} \frac{\mathrm{kg} \mathrm{U}}{\text { day }} \\
& \therefore \frac{5.46 \times 10^{-2} \mathrm{~kg} \mathrm{U} / \text { day }}{5708 \mathrm{~kg} \mathrm{U} / \mathrm{day}}=9.56 \times 10^{-6} \frac{\mathrm{kg} \mathrm{U} \text { (airborne) }}{\mathrm{kg} \mathrm{U} \text { (product) }}
\end{aligned}
$$

5-2. Assume only natural $U$ input to diffusion enrichment facility.

$$
\begin{aligned}
& (15, \mathrm{p} \cdot \mathrm{A}-259) \\
& 300 \frac{1 \mathrm{bs} U}{\mathrm{yr}} \times \frac{0.4536 \mathrm{~kg}}{1 \mathrm{~b}} \times \frac{\mathrm{yr}}{365 \text { days }}=3.73 \times 10^{-1} \frac{\mathrm{kg} \mathrm{U} \text { (1 iquid) }}{\text { day }}
\end{aligned}
$$


REFERENCE CALCULATIONS: Diffusion Enrichment Facility (cont'd)

$\therefore \frac{3.73 \times 10^{-1} \mathrm{~kg} \mathrm{U} / \text { day }}{5708 \mathrm{~kg} \mathrm{U} / \text { day }}=6.53 \times 10^{-5} \frac{\mathrm{kg} U \text { (1 iquid) }}{\mathrm{kgU} \text { (product) }}$

(15, p. A-259)

5-3. $\quad 5 \frac{\mathrm{Ci}^{234} \mathrm{Th}}{\mathrm{yr}} \times \frac{\mathrm{yr}}{365 \text { days }} \times \frac{\mathrm{g}^{234} \mathrm{Th}}{2.32 \times 10^{4} \mathrm{Ci}^{234} \mathrm{Th}} \times \frac{\mathrm{kg}}{10^{3} \mathrm{~g}}=5.90 \times 10^{-10} \frac{\mathrm{kg}^{234} \mathrm{Th}}{\text { day }}$

$\therefore \frac{5.90 \times 10^{-10} \mathrm{~kg}{ }^{234} \mathrm{Th} / \text { day }}{5708 \mathrm{~kg} \mathrm{U} / \text { day }}=1.03 \times 10^{-13} \frac{\mathrm{kg}^{234} \mathrm{Th}}{\mathrm{kg} \mathrm{U} \text { (product) }}$

(14, p. 3.3-10)

5-4. $7.56 \times 10^{4} \frac{\mathrm{\mu g} \mathrm{HF}}{\mathrm{sec}} \times \frac{86,400 \mathrm{sec}}{\text { day }} \times \frac{\mathrm{kg}}{10^{9} \mu \mathrm{g}}=6.53 \frac{\mathrm{kg} \mathrm{HF}}{\text { day }}$

$\therefore \frac{6.53 \mathrm{~kg} \mathrm{HF} / \text { day }}{5708 \mathrm{~kg} \mathrm{U} / \text { day }}=1.14 \times 10^{-3} \frac{\mathrm{kg} \mathrm{HF}}{\mathrm{kg} \mathrm{U} \text { (product) }}$

(16, p. 11)

5-5. $2,260 \frac{\text { ibs Freon }}{\text { month }} \times \frac{12 \text { months }}{365 \text { days }} \times \frac{\mathrm{kg}}{2.205 \mathrm{lbs}}=3.37 \times 10^{1} \frac{\mathrm{kg} \text { Freon }}{\text { day }}$

$\therefore \frac{3.37 \times 10^{1} \mathrm{~kg} \mathrm{Freon} / \text { day }}{5708 \mathrm{~kg} \mathrm{U} / \text { day }}=5.90 \times 10^{-3} \frac{\mathrm{kg} \mathrm{Freon}}{\mathrm{kg} \mathrm{U} \text { (product) }}$ 
REFERENCE CALCULATIONS: Diffusion Enrichment Facility (cont'd)

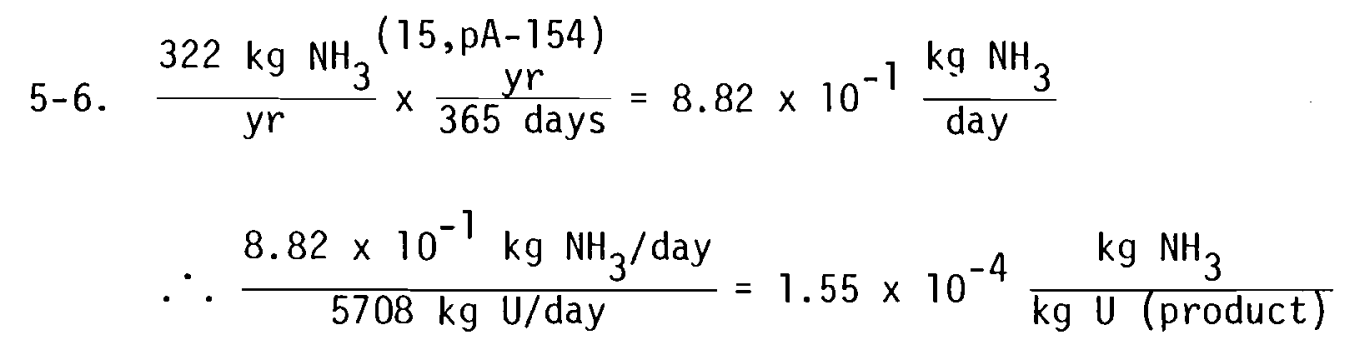

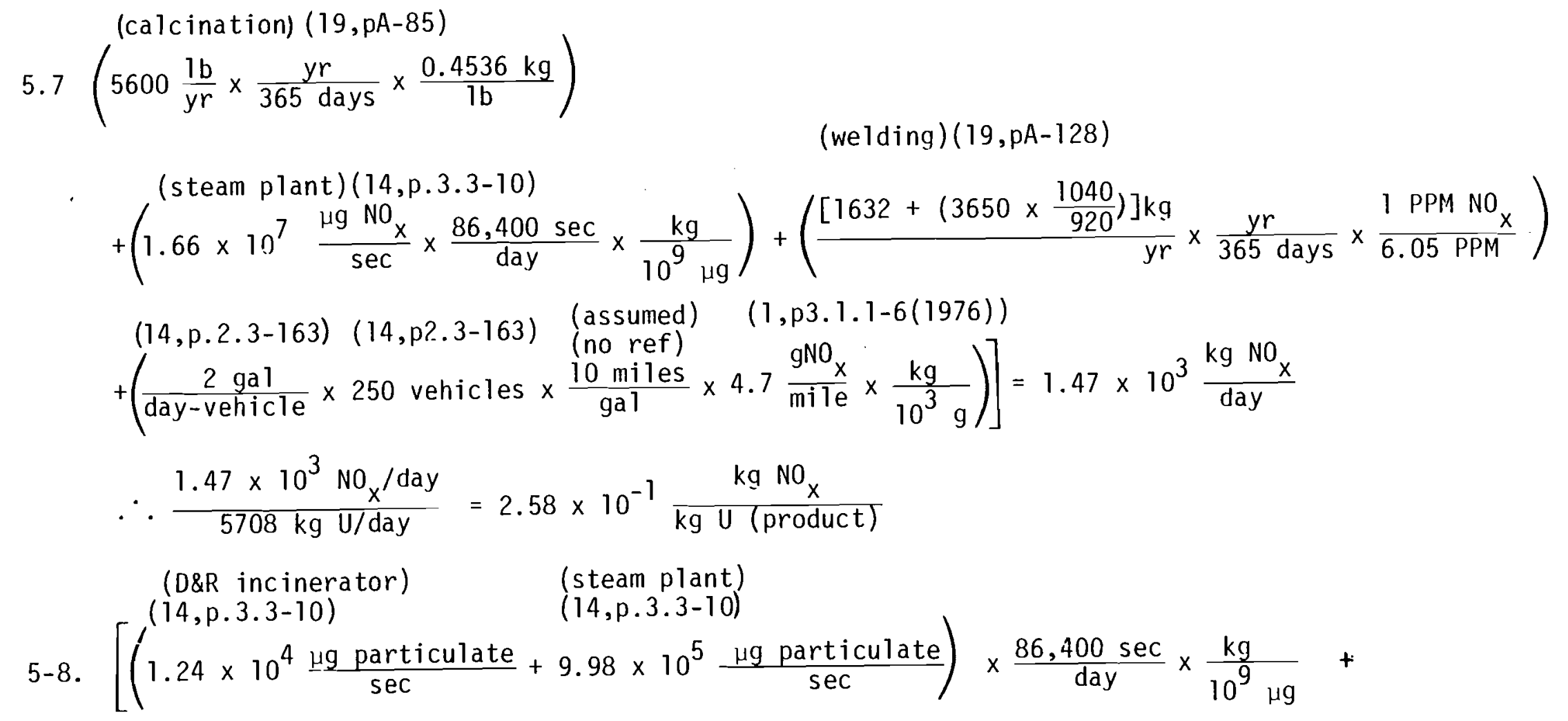


REFERENCE CALCULATIONS: Diffusion Enrichment Facility (cont'd)

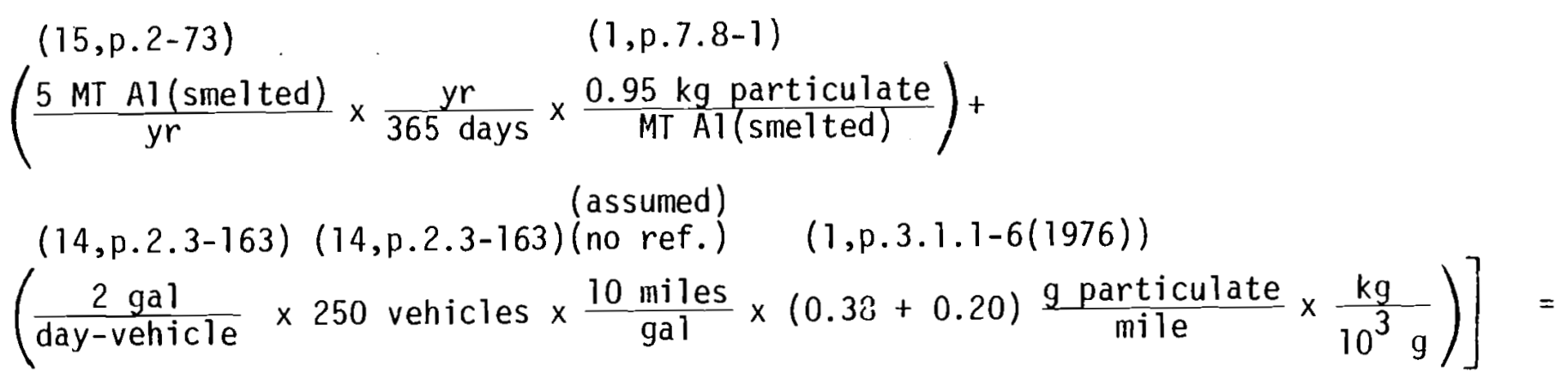

$9.02 \times 10^{1} \frac{\mathrm{kg} \text { particulate }}{\text { day }}$

i

G

$\therefore \frac{9.02 \times 10^{1} \mathrm{~kg} \text { particulate } / \text { day }}{5708 \mathrm{~kg} \mathrm{U} / \text { day }}=1.58 \times 10^{-2}-\frac{\mathrm{kg} \text { particulate }}{\mathrm{kg} U(\text { product })}$

(D\&R incinerator) (steam plant)

$(17$, p.3.3-10) $\quad(14$, p.3.3-10)

5-9. $\left[\left(1.45 \times 10^{2}-\frac{\mu \mathrm{g} \mathrm{S0}}{\mathrm{sec}}+2.08 \times 10^{7} \frac{\mu \mathrm{SO} 0_{\mathrm{x}}}{\mathrm{sec}}\right) \times \frac{86,400 \mathrm{sec}}{\mathrm{day}} \times \frac{\mathrm{kg}}{10^{9} \mu \mathrm{g}}+\right.$

$(14$, p.2.3-163)(14,p.2.3-163) (assumed) $(1$, p.3.1.1-6(1976))

$\left(\frac{2 \mathrm{gal}}{\text { day-vehicle }} \times 250\right.$ vehicles $\left.\left.\times \frac{10 \mathrm{miles}}{\mathrm{gal}} \times \frac{0.20 \mathrm{~g} \mathrm{S0}}{\mathrm{mile}} \times \frac{\mathrm{kg}}{10^{3} \mathrm{~g}}\right)\right]=1.80 \times 10^{3} \frac{\mathrm{kg} \mathrm{S0} \times}{\text { day }}$

$\therefore \frac{1.80 \times 10^{3} \mathrm{~kg} \mathrm{S0}{ }_{x} / \text { day }}{5708 \mathrm{~kg} \mathrm{U} / \mathrm{day}}=3.15 \times 10^{-1} \mathrm{~kg} \mathrm{S0_{x }}$ 
REFERENCE CALCULATIONS: Diffusion Enrichment Facility (cont'd)

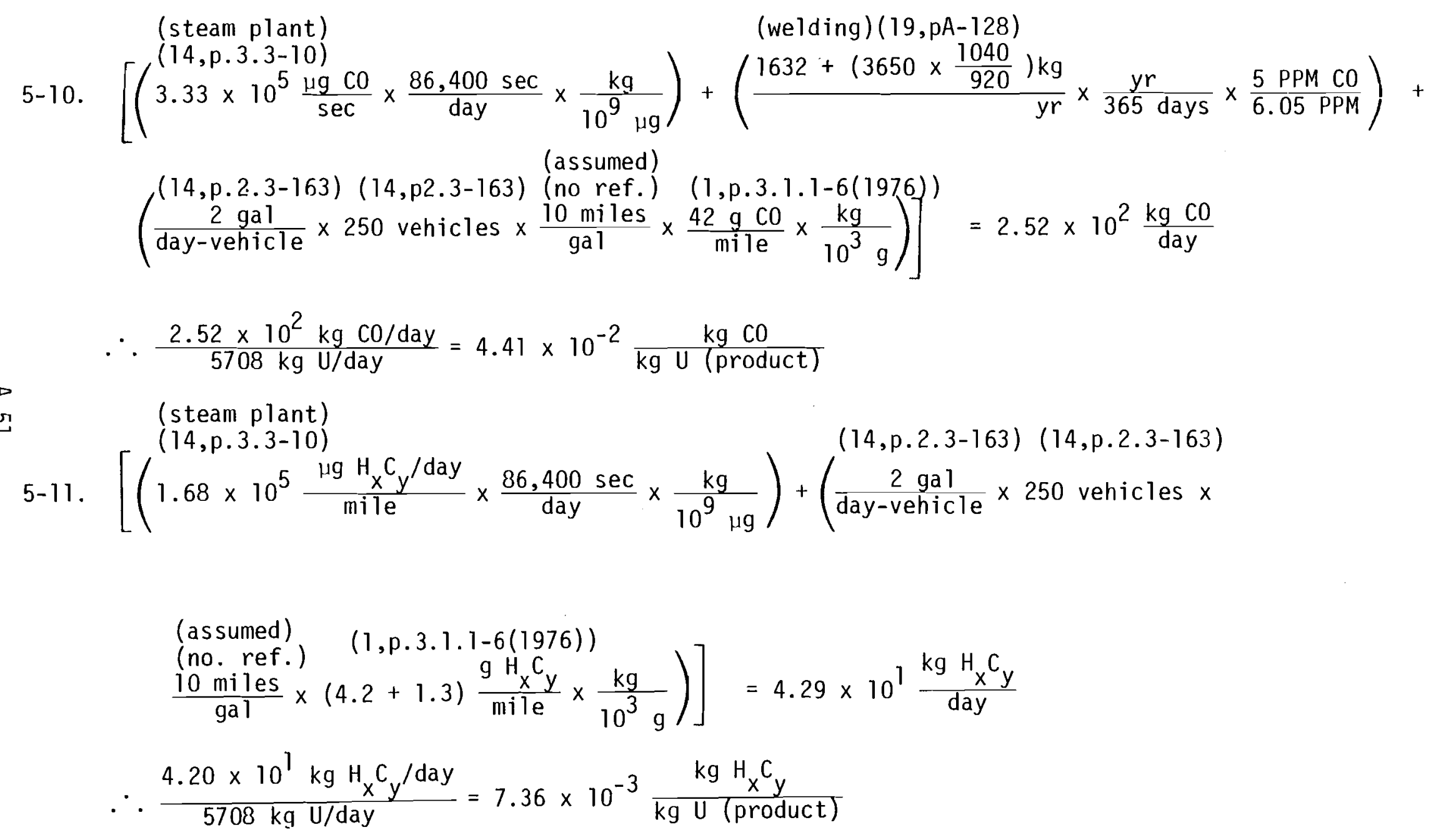


REFERENCE CALCULATIONS: Diffusion Enrichment Facility (cont'd)

$$
\begin{aligned}
& \text { (welding) } \\
& \text { 5-12. }\left(\begin{array}{l}
(19, \mathrm{pA}-128) \\
\left.1632+\left(3650 \times \frac{1040}{920}\right)\right)
\end{array}\right) \frac{\mathrm{kg}}{\mathrm{yr}} \times \frac{\mathrm{yr}}{365 \text { days }} \times \frac{0.05 \mathrm{PPM} 0_{3}}{6.05 \mathrm{PPM}}=1.30 \times 10^{-1} \frac{\mathrm{kg} 0_{3}}{\text { day }} \\
& \therefore \frac{1.30 \times 10^{-1} \mathrm{~kg} 0_{3} / \text { day }}{5708 \mathrm{~kg} \mathrm{U} / \text { day }}=2.28 \times 10^{-5} \mathrm{~kg} 0_{3} \\
& \therefore \frac{\mathrm{kg} \text { (product) }}{}
\end{aligned}
$$

5-13. Assume that Chlorothene is perchloroethylene $\left(\mathrm{Cl}_{2} \mathrm{C}=\mathrm{CCl}_{2}\right)$ with a density of $1.6 \mathrm{~g} / \mathrm{ml}$ (4,p.C-292) ( $\begin{aligned} & (19, \mathrm{pA}-128) \\ & \mathrm{i}\end{aligned} \frac{350 \mathrm{gal} \mathrm{ch} \text { lorothene }}{\mathrm{yr}} \times \frac{\mathrm{yr}}{365 \text { days }} \times \frac{3.7851 \mathrm{iter}}{\mathrm{gal}} \times \frac{10^{3} \mathrm{ml}}{1 \mathrm{iter}} \times \frac{1.6 \mathrm{~g}}{\mathrm{ml}} \times \frac{\mathrm{kg}}{10^{3} \mathrm{~g}}=5.81 \frac{\mathrm{kg} \mathrm{chlorothene}}{\text { day }}$

$\therefore \frac{5.81 \mathrm{~kg} \mathrm{chlorothene} / \text { day }}{5708 \mathrm{~kg} \mathrm{U} / \text { day }}=1.02 \times 10^{-3} \frac{\mathrm{kg} \mathrm{chlorothene}}{\mathrm{kg} \mathrm{U} \text { (product) }}$

5-14. $(7500+850) \frac{\text { gal trichloroethylene }}{y r} \times \frac{y r}{365 \text { days }} \times \frac{3.785 \text { liter }}{\text { gal }} \times \frac{10^{3} \mathrm{ml}}{\text { liter }} \times$

(assumed)

$(4$, p.C-292)

$\frac{1.5 \mathrm{~g}}{\mathrm{ml}} \times \frac{\mathrm{kg}}{10^{3} \mathrm{~g}}=1.30 \times 10^{2} \frac{\mathrm{kg} \text { trichloroethylene }}{\text { day }}$ 
REFERENCE CALCULATIONS: Diffusion Enrichment Facility (cont'd)

$\therefore \frac{1.30 \times 10^{2} \mathrm{~kg} \text { trichloroethylene } / \text { day }}{5708 \mathrm{~kg} \mathrm{U} / \text { day }}=2.28 \times 10^{-2} \mathrm{kg \text {trichloroethylene }}$

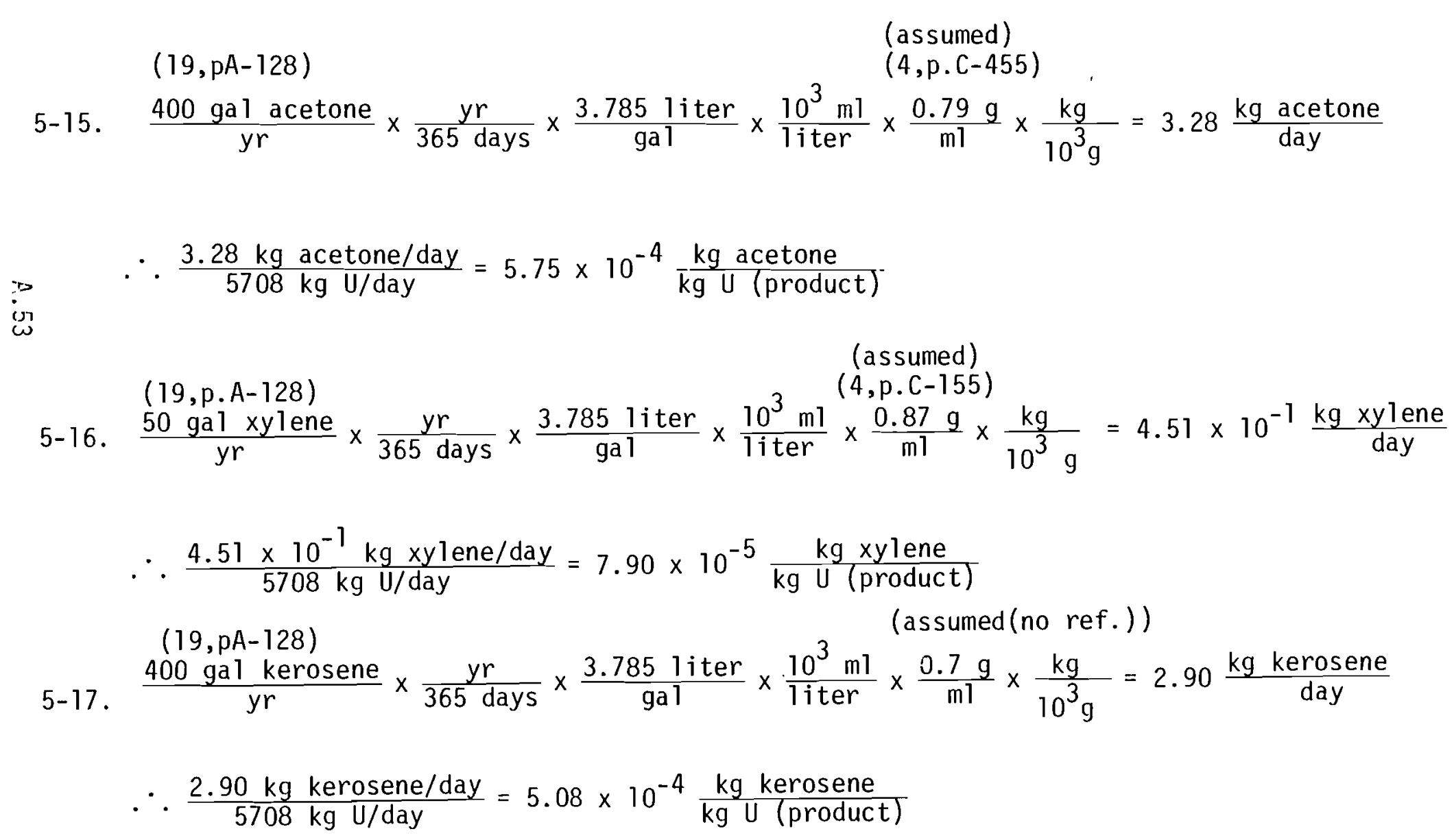


REFERENCE CALCULATIONS: Diffusion Enrichment Facility (cont'd)

$(14, p .2 .3-146)$

5-18. $5.3 \frac{\mathrm{mg} \mathrm{NO}}{\text { liter }} \times 1.71 \times 10^{7} \frac{\text { liters }}{\text { day }} \times \frac{\mathrm{kg}}{10^{6} \mathrm{mg}}=9.06 \times 10^{1} \frac{\mathrm{kg} \mathrm{NO}}{\mathrm{day}}$

$\therefore \frac{9.06 \times 10^{1} \mathrm{~kg} \mathrm{N0} / \text { day }}{5708 \mathrm{~kg} \mathrm{U/day}}=1.59 \times 10^{-2} \frac{\mathrm{kg} \mathrm{N0}}{3}$

(14, p.2.3-146)

5-19. $0.04 \frac{\mathrm{mg} \mathrm{Al}}{\text { liter }} \times 1.71 \times 10^{7} \frac{\text { liters }}{\text { day }} \times \frac{\mathrm{kg}}{10^{6} \mathrm{mg}}=6.84 \times 10^{-1} \frac{\mathrm{kg} \mathrm{Al}}{\text { day }}$
$\therefore \frac{6.84 \times 10^{-1} \mathrm{~kg} \mathrm{Al} / \text { day }}{5708 \mathrm{~kg} \mathrm{U} / \text { day }}=1.20 \times 10^{-4} \frac{\mathrm{kg} \mathrm{Al}}{\mathrm{kg} \mathrm{U( \text {product) }}}$

$(14, p .2 .3-146)$

5-20. $0.07 \frac{\mathrm{mg} \mathrm{F}}{\text { liter }} \times 1.71 \times 10^{7} \frac{\text { liters }}{\text { day }} \times \frac{\mathrm{kg}}{10^{6} \mathrm{mg}}=1.20 \frac{\mathrm{kg} \mathrm{F}}{\text { day }}$

$\therefore \frac{1.20 \mathrm{~kg} \mathrm{~F} / \text { day }}{5708 \mathrm{~kg} \mathrm{U} / \text { day }}=2.10 \times 10^{-4} \frac{\mathrm{kg} \mathrm{F}}{\mathrm{kg} \mathrm{U} \text { (product) }}$

$(14, p .2 .3-146)$

5-21. $0.01 \frac{\mathrm{mg} \text { tributylphosphate }}{1 \text { iter }} \times 1.71 \times 10^{7} \frac{1 \mathrm{iters}}{\text { day }} \times \frac{\mathrm{kg}}{10^{6} \mathrm{mg}}=1.71 \times 10^{-1} \frac{\mathrm{kg} \mathrm{TBP}}{\text { day }}$ 
REFERENCE CALCULATIONS: Diffusion Enrichment Facility (cont'd)

$\therefore \frac{1.71 \times 10^{-1} \mathrm{~kg} \text { tributylphosphate } / \text { day }}{5708 \mathrm{~kg} \mathrm{U} / \text { day }}=3.00 \times 10^{-5} \frac{\mathrm{kg} \mathrm{TBP}}{\mathrm{kg} \mathrm{U} \text { (product) }}$

5-22. $0.03 \frac{\text { mg varsol }}{1 \text { iter }} \times 1.71 \times 10^{7} \frac{\text { 1iters }}{\text { day }} \times \frac{\mathrm{kg}}{10^{6} \mathrm{mg}}=5.13 \times 10^{-1} \frac{\mathrm{kg} \text { varsol }}{\text { day }}$

$\therefore \frac{5.13 \times 10^{-1} \mathrm{~kg} \mathrm{varsol} / \text { day }}{5708 \mathrm{~kg} \mathrm{U} / \text { day }}=8.99 \times 10^{-5} \frac{\mathrm{kg} \text { varsol }}{\mathrm{kg} \mathrm{U} \text { (product) }}$

in

in

5-23. $\quad 0.70 \frac{\mathrm{mg} \mathrm{P0}_{4}}{1 \text { iter }} \times 1.71 \times 10^{7} \frac{\text { liters }}{\text { day }} \times \frac{\mathrm{kg}}{10^{6} \mathrm{mg}}=1.20 \times 10^{1} \frac{\mathrm{kg} \mathrm{PO}_{4}}{\text { day }}$

$\therefore \frac{1.20 \times 10^{1} \mathrm{~kg} \mathrm{P0} / \text { day }}{5708 \mathrm{~kg} \mathrm{U} / \text { day }}=2.10 \times 10^{-3} \frac{\mathrm{kg} \mathrm{PO}_{4}}{\mathrm{~kg} \mathrm{U} \text { (product) }}$

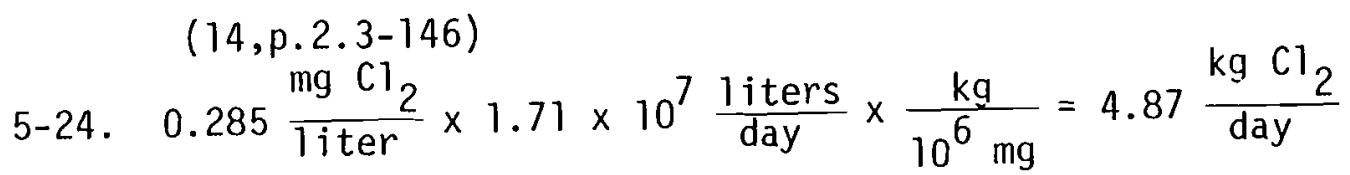

$\therefore \frac{4.87 \mathrm{~kg} \mathrm{Cl}_{2} / \text { day }}{5708 \mathrm{~kg} \mathrm{U} / \text { day }}=8.53 \times 10^{-4} \frac{\mathrm{kg} \mathrm{Cl} 2}{\mathrm{~kg} \mathrm{U} \text { (product) }}$ 


\section{REFERENCE CALCULATIONS: Diffusion Enrichment Facility (cont'd)}

$5-02-77$

$$
\begin{aligned}
& \quad(14, \mathrm{p} .2 .3-146) \\
& \text { 5-25. } 0.047 \frac{\mathrm{mg} \mathrm{Cr}}{1 \text { iter }} \times 1.71 \times 10^{7} \frac{\text { liters }}{\text { day }} \times \frac{\mathrm{kg}}{10^{6} \mathrm{mg}}=8.04 \times 10^{-1} \frac{\mathrm{kg} \mathrm{Cr}}{\text { day }} \\
& \therefore \frac{8.04 \times 10^{-1} \mathrm{~kg} \mathrm{Cr} / \text { day }}{5708 \mathrm{~kg} \mathrm{U} / \text { day }}=1.41 \times 10^{-4} \frac{\mathrm{kg} \mathrm{Cr}}{\mathrm{kg} \mathrm{U}(\text { product })}
\end{aligned}
$$

$(14, p .2 .3-146)$

5-26. $0.475 \frac{\mathrm{mg} \mathrm{Zn}}{1 \mathrm{iter}} \times 1.71 \times 10^{7} \frac{\text { liters }}{\text { day }} \times \frac{\mathrm{kg}}{10^{6} \mathrm{mg}}=8.12 \frac{\mathrm{kg}}{\mathrm{day}}$

$\therefore \frac{8.12 \mathrm{~kg} \mathrm{Zn/day}}{5708 \mathrm{~kg} \mathrm{U} / \mathrm{day}}=1.42 \times 10^{-3} \frac{\mathrm{kg} \mathrm{Zn}}{\mathrm{kg} \mathrm{U}(\text { product })}$

5-27. Assuming the same ratio of ${ }^{238} \mathrm{U}:{ }^{235} \mathrm{U}:{ }^{234} \mathrm{U}$ as is indicated in (14, p.2.3-50 and p.2.3-140) then:

$$
\begin{aligned}
& \text { (from ref.ralc. 5-1) (inferred from ref.calc.5-1) } \\
& \therefore \quad 9.56 \times 10^{-6} \frac{\mathrm{kg} \mathrm{U}(\text { airborne })}{\mathrm{kg} \mathrm{U}(\text { prod-output })} \times \frac{1.92 \times 10^{4} \mathrm{~g}{ }^{238} \mathrm{U} / \mathrm{yr}}{1.99 \times 10^{4} \mathrm{~g} \mathrm{U(235)/ \textrm {yr }}}=\frac{9.22 \times 10^{-6} \mathrm{~kg}{ }^{238} \mathrm{U}(\mathrm{air})}{\mathrm{kg} \mathrm{U}(\text { product })}
\end{aligned}
$$


REFERENCE CALCULATIONS: Diffusion Enrichment Facility (cont'd)

$$
\begin{aligned}
& \text { (from ref.calc. 5-1) (inferred from ref.calc.5-1) }
\end{aligned}
$$

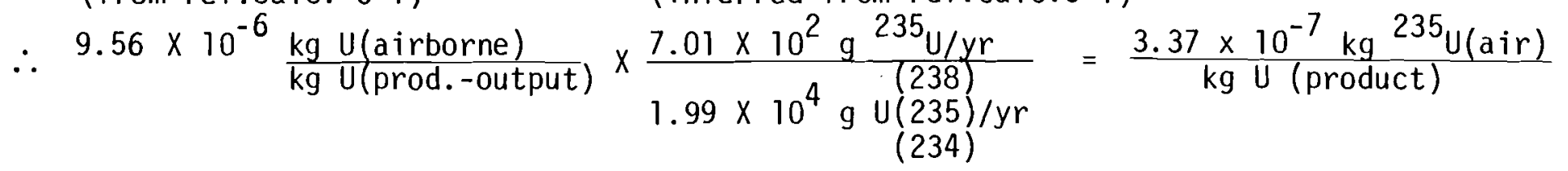

$$
\begin{aligned}
& \text { (from ref.calc.5-1) (inferred from ref.calc.5-1) } \\
& \therefore 9.56 \times 10^{-6} \frac{\mathrm{kg} \mathrm{U} \text { (airborne) }}{\mathrm{kgU} \text { (prod. output) }} \times \frac{3.17 \times 10^{-4} \mathrm{~g}^{234} \mathrm{U} / \mathrm{yr}}{1.99 \times 10^{4} \mathrm{gU}(238)}=\frac{1.52 \times 10^{-13} \mathrm{~kg} / \mathrm{yr}}{\mathrm{kgU} \text { (product) }}=\frac{(\text { air) }}{234}
\end{aligned}
$$

5-28. Assuming the same ratio of ${ }^{238} U:{ }^{235} U:{ }^{234} U$ as is indicated in (14, p.2.3-141) then:

$$
\begin{aligned}
& \text { (from ref.calc.5-2) (inferred from 14, p.2.3-141) }
\end{aligned}
$$

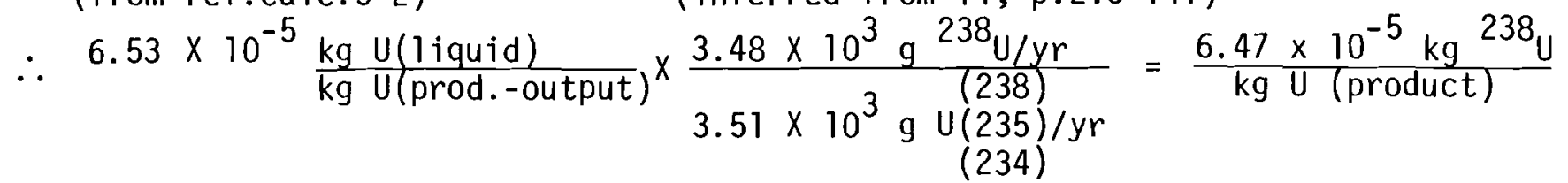

$$
\begin{aligned}
& \text { (from ref.calc. 5-2) (inferred from 14, p.2.3-141) } \\
& \therefore 6.53 \times 10^{-5} \frac{\mathrm{kg} \mathrm{U}(1 \mathrm{qquid})}{\mathrm{kgU} \text { (prod. -output) }} \times \frac{2.77 \times 10^{1} \mathrm{~g} 235 \mathrm{U} / \mathrm{yr}}{3.51 \times 10^{3} \mathrm{gU}(238) \mathrm{(235)/ \textrm {yr }}}=\frac{5.15 \times 10^{-7} \mathrm{~kg}{ }^{235} \mathrm{U}}{\mathrm{kg} \mathrm{U} \text { (product) }}
\end{aligned}
$$

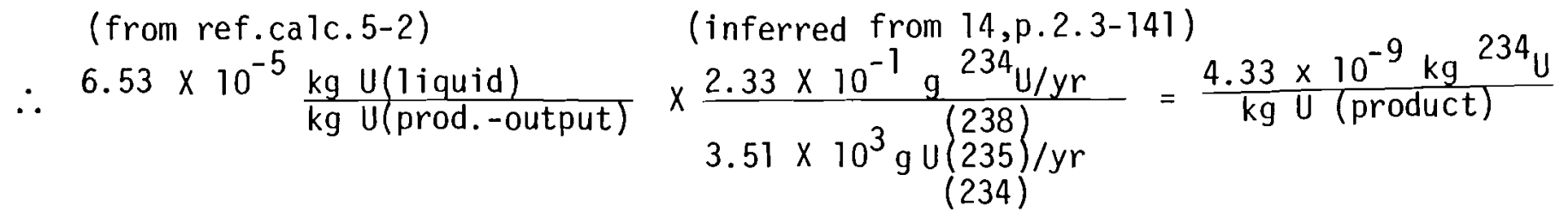




\begin{tabular}{|c|c|c|c|c|c|c|}
\hline \multirow[b]{2}{*}{ Material Released } & \multicolumn{3}{|c|}{ Air Borne } & \multicolumn{3}{|c|}{ Liquid } \\
\hline & Factor & Reliability & $\begin{array}{l}\text { Ref. } \\
\text { Calc. }\end{array}$ & Factor & Reliability & $\begin{array}{l}\text { Ref. } \\
\text { Calc. }\end{array}$ \\
\hline Uranium (enriched $3.2 \%$ ) & $1 \times 10^{-6}$ & 2 & $6-1$ & $1 \times 10^{-7}$ & 2 & $6-2$ \\
\hline \multicolumn{7}{|l|}{ Thor ium - 234} \\
\hline \multicolumn{7}{|l|}{ Protactinium - 234} \\
\hline Hydrogen Fluoride (HF) & $1 \times 10^{-4}$ & 2 & $6-4$ & & & \\
\hline Ammon ium Fluor ide $\left(\mathrm{NH}_{4} \mathrm{~F}\right)$ & $1 \times 10^{-4}$ & 2 & $6-5$ & & & \\
\hline Ammonia $\left(\mathrm{NH}_{3}\right)$ & $2 \times 10^{-1}$ & 2 & $6-6$ & $2 \times 10^{-3}$ & 2 & $6-22$ \\
\hline Nitrogen $0 x$ ides $\left(\mathrm{NO}_{\mathrm{x}}\right)$ & $2 \times 10^{-3}$ & 1 & $6-7$ & & & \\
\hline Nitric Acid $\left(\mathrm{HNO}_{3}\right)$ & $5 \times 10^{-3}$ & 1 & $6-8$ & & & \\
\hline \multicolumn{7}{|l|}{ Hydrogen $\left(\mathrm{H}_{2}\right)$} \\
\hline \multicolumn{7}{|l|}{ Helium (He) } \\
\hline Hydrocarbons & $1 \times 10^{-6}$ & 2 & $6-9$ & & & \\
\hline Sulfur oxides $\left(\mathrm{SO}_{\mathrm{x}}\right)$ & $2 \times 10^{-7}$ & 2 & $6-9$ & & & \\
\hline \multicolumn{7}{|l|}{ Nonradioact ive } \\
\hline Particulate & $7 \times 10^{-6}$ & 2 & $6-9$ & & & \\
\hline Carbon Monoxide ( $\mathrm{CO})$ & $7 \times 10^{-6}$ & 2 & $6-9$ & & & \\
\hline \multicolumn{7}{|l|}{ Sulfuric Acid $\left(\mathrm{H}_{2} \mathrm{SO}_{4}\right)$} \\
\hline \multicolumn{7}{|l|}{ Bor ic Ac id $\left(\mathrm{H}_{3} \mathrm{BO}_{3}\right)$} \\
\hline \multicolumn{7}{|l|}{ Nickel Sulfate $\left(\mathrm{NiSO}_{4}\right)$} \\
\hline \multicolumn{7}{|l|}{ Nickel Chloride $\left(\mathrm{NiCl}_{2}\right)$} \\
\hline Silver $(A g)$ & & & & $6 \times 10^{-5}$ & 2 & $6-10$ \\
\hline Iron (Fe) & & & & $3 \times 10^{-4}$ & 2 & $6-11$ \\
\hline Sodium $(\mathrm{Na})$ & & & & $2 \times 10^{-2}$ & 2 & $6-12$ \\
\hline
\end{tabular}


$\mathrm{U0}_{2}$ FUEL FARBICATION (cont'd) CHEMICAL RELEASE FACTOR $\mathrm{Kg} / \mathrm{Kg} U$ (INPUT)

\begin{tabular}{|c|c|c|c|c|c|c|}
\hline \multirow[b]{2}{*}{ Material Released } & \multicolumn{3}{|c|}{ Air Borne } & \multicolumn{3}{|c|}{ Liquid } \\
\hline & Factor & Reliability & $\begin{array}{l}\text { Ref. } \\
\text { Calc. }\end{array}$ & Factor & Reliability & $\begin{array}{l}\text { Ref. } \\
\text { Calc. }\end{array}$ \\
\hline Calcium (Ca) & & & & $4 \times 10^{-1}$ & 2 & $6-13$ \\
\hline Magnesium (Mg) & & & & $9 \times 10^{-4}$ & 2 & $6-14$ \\
\hline Manganese $(M n)$ & & & & $2 \times 10^{-5}$ & 2 & $6-15$ \\
\hline Molybdenun (Mo) & & & & $7 \times 10^{-5}$ & 2 & $6-16$ \\
\hline Nickel (Ni) & & & & $2 \times 10^{-4}$ & 2 & $6-17$ \\
\hline Boron (B) & & & & $3 \times 10^{-4}$ & 2 & $6-18$ \\
\hline Chloride $(\mathrm{Cl})$ & & & & $2 \times 10^{-2}$ & 2 & $6-19$ \\
\hline Phosphorus (P) & & & & $5 \times 10^{-4}$ & 2 & $6-20$ \\
\hline Nitrogen (KJELDAHL) & & & & $9 \times 10^{-2}$ & 2 & $2-21$ \\
\hline Floride (F) & & & & $3 \times 10^{-3}$ & 2 & $2-23$ \\
\hline Sulfate $\left(\mathrm{SO}_{4}\right)$ & & & & $3 \times 10^{-2}$ & 2 & $2-24$ \\
\hline Sulfite $\left(\mathrm{SO}_{3}\right)$ & & & & $4 \times 10^{-4}$ & 2 & $2-25$ \\
\hline \multicolumn{7}{|l|}{ Sulfide (S) } \\
\hline \multicolumn{7}{|l|}{ Phenols } \\
\hline $0 i l s$ and Grease & & & & $1 \times 10^{-3}$ & 2 & $2-26$ \\
\hline Hardness as $\mathrm{CaCO}_{3}$ & & & & $2 \times 10^{-1}$ & 2 & $6-27$ \\
\hline Ace tone & $x$ & & & & & \\
\hline Nitrogen & $x$ & & & & & \\
\hline Argon & $x$ & & & & & \\
\hline Zinc $(Z n)$ & & & & $x$ & & \\
\hline
\end{tabular}


U02 FUEL FARBICATION RADIONUCLIDE RELEASE FACTOR $\mathrm{Kg} / \mathrm{Kg} U$ (INPUT)

\begin{tabular}{|c|c|c|c|c|c|c|}
\hline \multirow[b]{2}{*}{ Material Released } & \multicolumn{3}{|c|}{ Air Borne } & \multicolumn{3}{|c|}{ Liquid } \\
\hline & Factor & Reliability & $\begin{array}{l}\text { Ref. } \\
\text { Calc. }\end{array}$ & Factor & Reliability & $\begin{array}{l}\text { Ref. } \\
\text { Calc. }\end{array}$ \\
\hline \multicolumn{7}{|c|}{ Uranium (enriched 3.2\%) } \\
\hline *Uranium - 238 & $1.13 \times 10^{-6}$ & 2 & $6-28$ & $1.15 \times 10^{-7}$ & 2 & $6-29$ \\
\hline *Uranium - 235 & $3.74 \times 10^{-8}$ & 2 & $6-28$ & $3.81 \times 10^{-9}$ & 2 & $6-29$ \\
\hline *Uranium - 234 & $1.79 \times 10^{-10}$ & 2 & $6-28$ & $1.82 \times 10^{11}$ & 2 & $6-29$ \\
\hline
\end{tabular}

*Thor ium - 234

$\star$ Protactinium - 234

* Has Data Base In Computer Mode 1 


\section{REFERENCE CALCULATIONS: $\quad \underline{\cup} \underline{0}_{2}$ FUEL FABRICATION FACILITY (365 operating Days/yr)}

6-1. U input to the $\mathrm{UO}_{2}$ fuel fabrication facility:

(assumed from reference 18)

$4404 \frac{\mathrm{kg} \mathrm{U}}{\mathrm{day}}$ (enriched to $3.2 \% 235 \mathrm{U}$ )

$U$ airborne output from the $\mathrm{UO}_{2}$ fuel fabrication facility (from refereme 18):

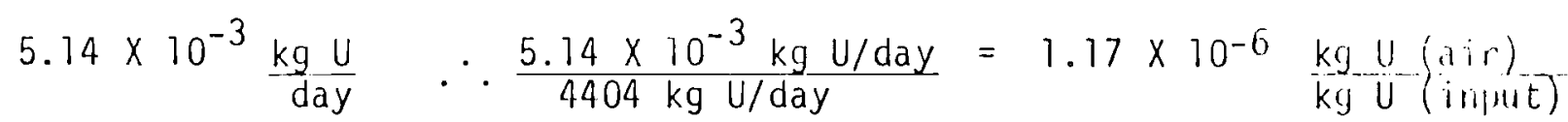

(From reference 18 pages 3.3-14 and 4.8-13:)

6-2. $\frac{5.26 \times 10^{-4} \mathrm{~kg} \mathrm{U/day}}{4404 \mathrm{kgU} / \mathrm{day}}=1.19 \times 10^{-7} \frac{\mathrm{kgU}(1 \text { iquid) }}{\mathrm{kgU} \text { (input) }}$

$$
(18, \text { p.3.3-10) (18, p.3.3-10) }
$$

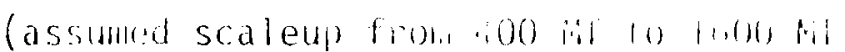
only increases F remise by $/ 1 \cdot,+$

6-3. $0.36 \frac{\mathrm{mgF}}{\mathrm{m}^{3}} \times \frac{\mathrm{m}^{3}}{10^{6} \mathrm{mi}}$ $\times 6.0 \times 10^{10} \frac{\mathrm{ml}}{\mathrm{hr}} \times \frac{24 \mathrm{hr}}{\mathrm{day}} \times \frac{\mathrm{kg}}{10^{6} \mathrm{mg}} \times \frac{7}{5}$
$=7.26 \times 10^{-1} \mathrm{~kg} \frac{1}{\text { day }}$

* Phone call from Roger E. Fischer, Radiological \& Environmental Engineer fur Westinghouse ius lari lu, Division, Columbia, South Carolina, on April 15, 1977 provided the information that tileir llulired scale-up from 400 MTU/year to 1600 MTU/year would not increase the Fluorine to the envirwalent. lir l.an apparent 4 to 7 but only by the ratio of 7 to 5 due to easier design of valve activator liskings, etc., for the larger equipment. 
REFERENCE CALCULATIONS: $\mathrm{UO}_{2}$ Fuel Fabrication Facility (cont'd)

(from ref.

(calc. 6-3)
6-4. $7.26 \times 10^{-1} \frac{\mathrm{kgF}}{\text { day }} \times \frac{\mathrm{kg}-\mathrm{mole} \mathrm{F}}{19 \mathrm{~kg} \mathrm{~F}} \times \frac{2 / 3 \mathrm{~kg} \text {-mole HF}}{1 \mathrm{~kg}-\mathrm{mole} \mathrm{F}} \times \frac{20 \mathrm{~kg} \mathrm{HF}}{\mathrm{kg}-\mathrm{mole} \mathrm{HF}}=5.09 \times 10^{-1} \frac{\mathrm{kg} \mathrm{HF}}{\text { day }}$

$\therefore \frac{5.09 \times 10^{-1} \mathrm{~kg} \mathrm{HF} / \text { day }}{4404 \mathrm{~kg} \mathrm{U} / \text { day }}=1.16 \times 10^{-4} \frac{\mathrm{kg} \mathrm{HF}}{\mathrm{kg} \mathrm{U}(\text { input })}$

(from ref.

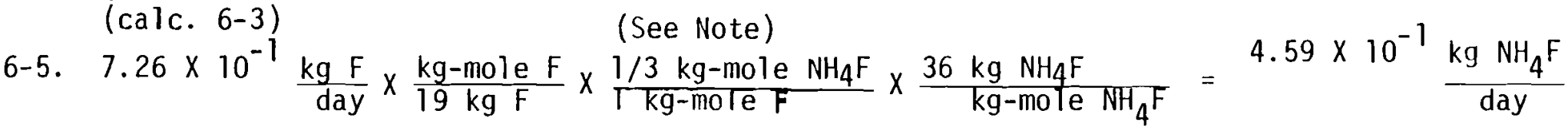

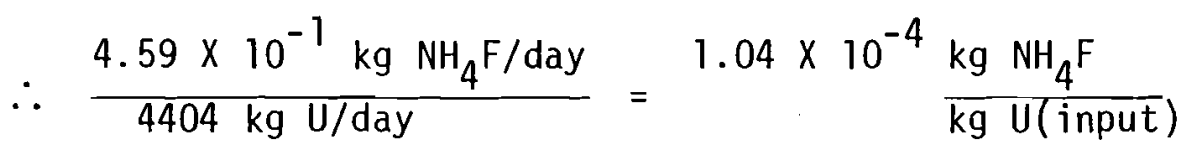

$(18$, p. 3.3-10)

6-6. $390 \frac{\mathrm{mg} \mathrm{NH}}{\mathrm{m}^{3}} \times \frac{\mathrm{m}^{3}}{10^{6} \mathrm{ml}} \times 6.0 \times 10^{10} \frac{\mathrm{ml}}{\mathrm{hr}} \times \frac{24 \mathrm{hr}}{\text { day }} \times \frac{\mathrm{kg}}{10^{6} \mathrm{mg}} \times \frac{7^{*}}{5}=\frac{7.86 \times 10^{2} \mathrm{~kg} \mathrm{NH} 3}{\text { day }}$

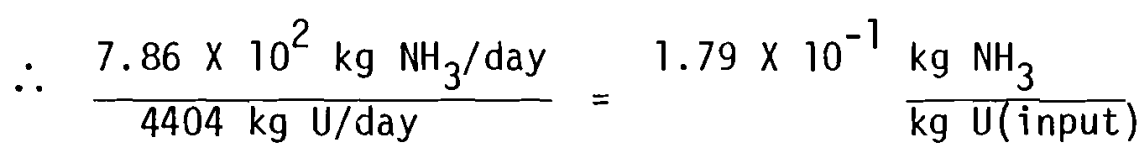

* assumed scaleup from $400 \mathrm{MT} /$ day to $1600 \mathrm{MT} /$ day only increases release by a ratio of $\frac{7}{5}$ (see calculation 6 -3) 
6-7. $\frac{(\text { See Note) }}{10 \mathrm{~kg} \mathrm{N0} / \text { day }}=2.27 \times 10^{-3} \mathrm{~kg} \mathrm{NO} \frac{x}{\mathrm{~kg} \mathrm{U( \text {input } )}}$

(See Note)

6-8. $24 \mathrm{~kg} \mathrm{HNO} /$ day $440 \overline{k g ~ U / d a y}$

$$
5.45 \times 10^{-3} \frac{\mathrm{kg} \mathrm{HNO}}{3}
$$

6-9. Basis for the following numbers is the use of $1.4 \times 10^{6} \mathrm{ft}^{3}$ natural gas per year at $1600 \mathrm{Mtu} / \mathrm{yr}$ is capacity (18, p.3.2-13,4.3-1) and EPA emission factors (1, p.1.4-2) - industrial boiler.

$$
\begin{aligned}
& \therefore \frac{5 \times 10^{-3} \mathrm{~kg} \mathrm{hydrocarbons} / \text { day }}{4404 \mathrm{~kg} \mathrm{U} / \text { day }}=1.14 \times 10^{-6} \frac{\mathrm{kg} \text { hydrocarbons }}{\mathrm{kg} \mathrm{U(input)}}
\end{aligned}
$$

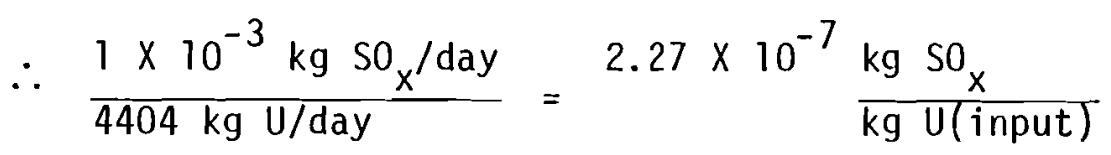

$$
\begin{aligned}
& \therefore \frac{3 \cdot \times 10^{-2} \mathrm{~kg} \text { particulate } / \text { day }}{4404 \mathrm{~kg} \mathrm{U/day}}=6.81 \times 10^{-6} \frac{\mathrm{kg} \text { particulate }}{\mathrm{kg} U(\text { input })}
\end{aligned}
$$

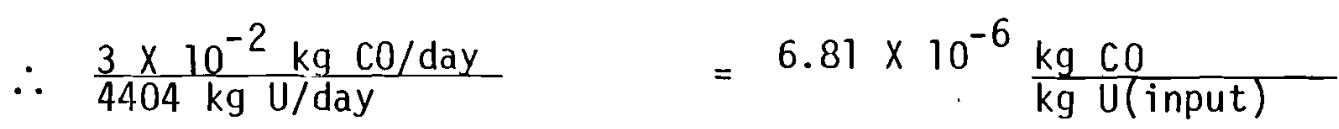


REFERENCE CALCULATIONS: $\mathrm{UO}_{2}$ Fuel Fabrication Facility (cont'd)

(See Note)

6-10. $\frac{0.25 \mathrm{~kg} \mathrm{Ag} / \text { day }}{4404 \mathrm{~kg} \mathrm{U} / \text { day }}=5.68 \times 10^{-5} \frac{\mathrm{kg} \mathrm{Ag}}{\mathrm{kg} \mathrm{U}(\text { input })}$

(See Notr:)

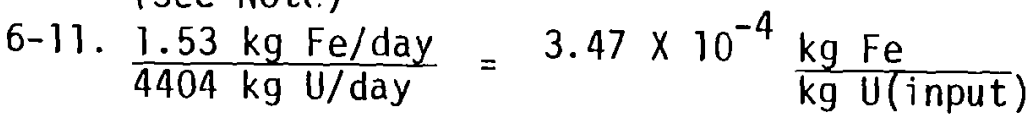

(See Note)

6-12. $\frac{102 \mathrm{~kg} \mathrm{Na} / \text { day }}{4404 \mathrm{~kg} \mathrm{U/day}}=2.32 \times 10^{-2} \frac{\mathrm{kg} \mathrm{Na}}{\mathrm{kg} \mathrm{U}(\text { input })}$

(See Note)

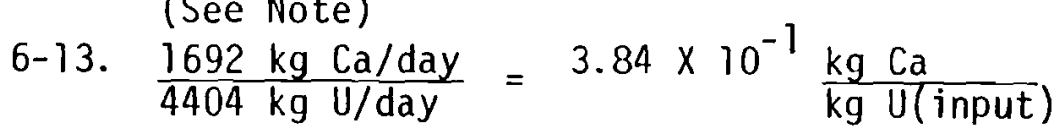

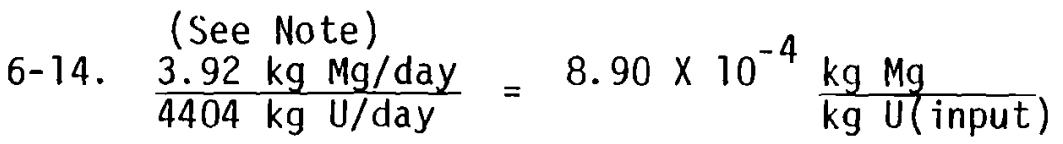

(See Note)

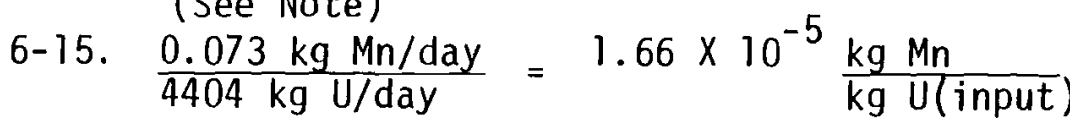


REFERENCE CALCULATIONS: $\mathrm{UO}_{2}$ Fuel Fabrication Facility (cont'd)

$$
\begin{aligned}
& \text { 6-16. } \begin{array}{l}
\text { (See Note) } \\
\frac{0.33 \mathrm{~kg} \mathrm{Mo/day}}{4404 \mathrm{~kg} \mathrm{U} / \text { day }}=7.49 \times 10^{-5} \frac{\mathrm{kg} \mathrm{Mo}}{\mathrm{kg} \mathrm{U}(\text { input })}
\end{array} \\
& \text { 6-17. } \frac{\text { (See Note) }}{\frac{0.7 \mathrm{~kg} \mathrm{Ni} / \text { day }}{4404 \mathrm{~kg} \mathrm{U/day}}}=1.59 \times 10^{-4} \frac{\mathrm{kg} \mathrm{Ni}}{\mathrm{kg} \mathrm{U}(\text { input })} \\
& \text { 6-18. } \frac{\text { (See Note) }}{\frac{1.23 \mathrm{~kg} \mathrm{~B} / \text { day }}{4404 \mathrm{~kg} \mathrm{U.day}}}=2.79 \times 10^{-4} \frac{\mathrm{kg} \mathrm{B}}{\mathrm{kg} \mathrm{U} \text { (input) }}
\end{aligned}
$$

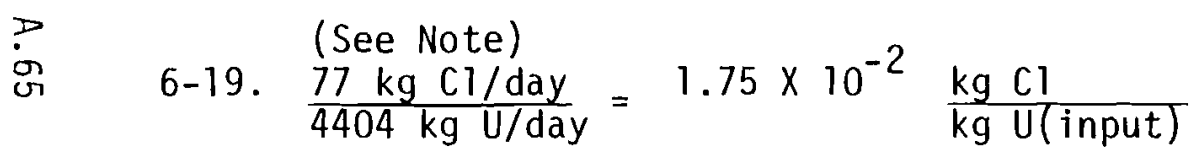

$$
\begin{aligned}
& \text { 6-20. } \frac{\text { (See Note) }}{\frac{2.03 \mathrm{~kg} \mathrm{P} / \text { day }}{4404 \mathrm{~kg} \mathrm{U} / \text { day }}}=4.61 \times 10^{-4} \frac{\mathrm{kg} \mathrm{P}}{\mathrm{kg} \mathrm{U}(\text { input })} \\
& \text { 6-21. } \frac{\text { (See Note) }}{\frac{388 \mathrm{~kg} \mathrm{~N} / \text { day }}{4404 \mathrm{~kg} \mathrm{U} / \text { day }}}=8.81 \times 10^{-2} \frac{\mathrm{kg} \mathrm{N}}{\mathrm{kg} \mathrm{U} \text { (input) }}
\end{aligned}
$$


REFERENCE CALCULATIONS: $\mathrm{uO}_{2}$ Fuel Fabrication Facility (cont'd)

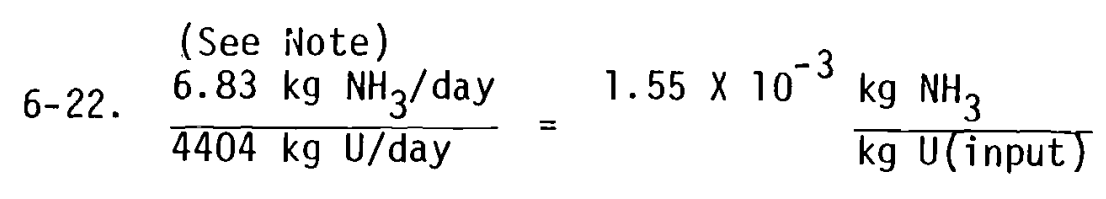

(See Note)

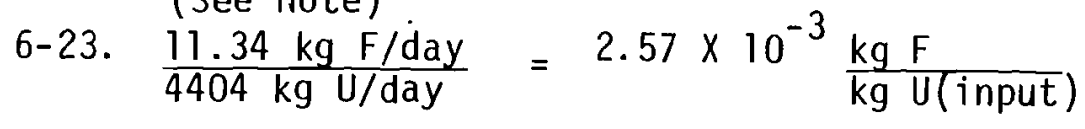

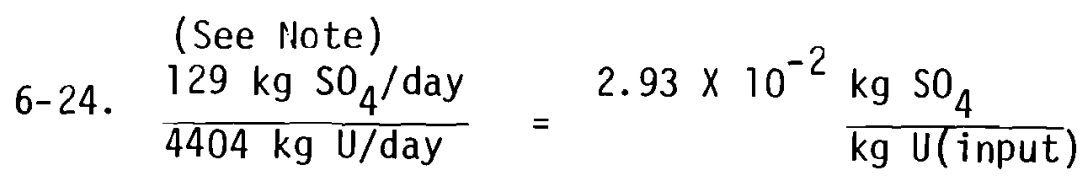

(See Note)

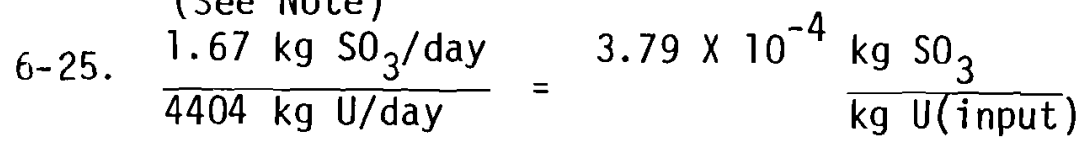

(See Note)

6-26. $\frac{5.67 \mathrm{~kg} \mathrm{oil} \text { and grease/day }}{4404 \mathrm{~kg} \mathrm{U} / \text { day }}=1.29 \times 10^{-3} \frac{\mathrm{kg} \mathrm{oil} \text { and grease }}{\mathrm{kg} \mathrm{U(input)}}$

(See Note)

6-27. $730.4 \mathrm{~kg} \mathrm{Ca} \mathrm{CO} /$ day $=1.66 \times 10^{-1} \mathrm{~kg} \mathrm{Ca} \mathrm{CO} 3$ $\overline{4404 \mathrm{~kg} \mathrm{U} / \mathrm{day}}=\overline{\mathrm{kg} \mathrm{U} \text { (input) }}$ 
REFERENCE CALCULATIONS: $\mathrm{UO}_{2}$ Fuel Fabrication Facility (cont'd)

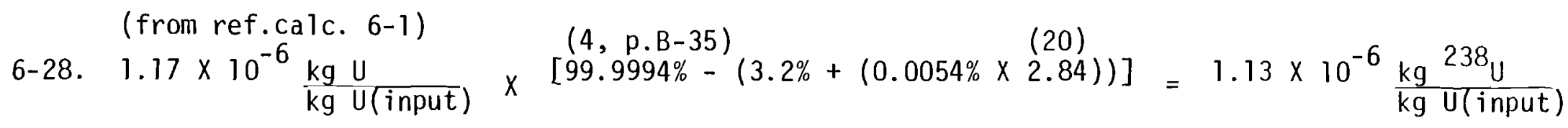

(from ref.calc. 6-1)

$1.17 \times 10^{-6} \frac{\mathrm{kg} \mathrm{U}}{\mathrm{kg} U(\text { input })} \times 3.2 \%=3.74 \times 10^{-8} \frac{\mathrm{kg}^{235} \mathrm{U}}{\mathrm{kg} \mathrm{U( \text {input } )}}$

(from ref.calc.6-1)

$1.17 \times 10^{-6} \mathrm{~kg} \mathrm{U}$

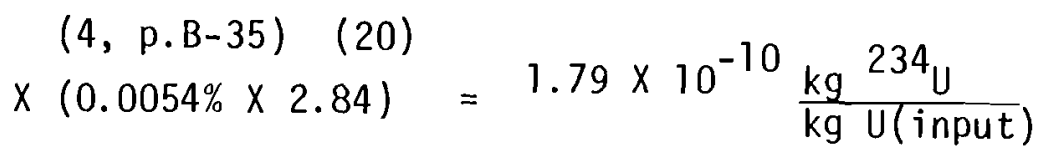

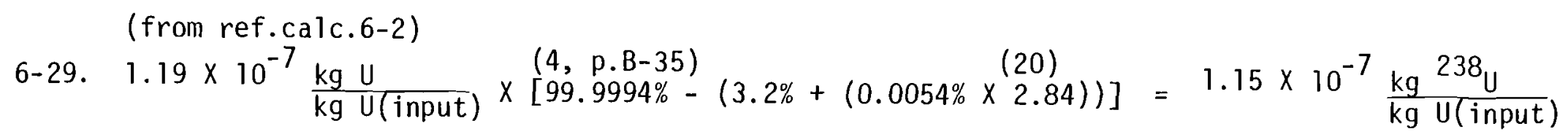

1

(from ref.calc. 6-2)

$1.19 \times 10^{-7} \frac{\mathrm{kg} \mathrm{U}}{\mathrm{kg} \mathrm{U}(\text { input })} \times 3.2 \%=3.81 \times 10^{-9} \frac{\mathrm{kg}^{235} \mathrm{U}}{\mathrm{kg} \mathrm{U}(\text { input })}$

(from ref.calc. 6-2)

$1.19 \times 10^{-7} \frac{\mathrm{kgU}}{\mathrm{kgU} \text { (input) }} \times\left(\begin{array}{l}(4, \mathrm{p} . \mathrm{B}-35) \\ (0.0054 \% \times 2.84)\end{array}=1.82 \times 10^{-11} \frac{\mathrm{kg} 234 \mathrm{U}}{\mathrm{kg} \mathrm{U} \text { (input) }}\right.$

NOTE: Many of the references used in this section were obtained through use of the references and other extensively footnoted data contained in the 800 pages of: "Description and Analysis of Reference Fuel Fabrication Facilities for LWR $\mathrm{UO}_{2}$ and $\mathrm{UO}_{2}--\mathrm{PuO}_{2}$ Fuels" prepared by Wendell $\mathrm{J}$. Bailley, Battelle Pacific Northwest Laboratories. Unfortunately, it hâs not as yet been released for publication, and therefore cannot be used as a direct reference. 
MOX FUEL FABRICATION CHEMICAL RELEASE FACTOR $\mathrm{Kg} / \mathrm{Kg} U$ (INPUT)

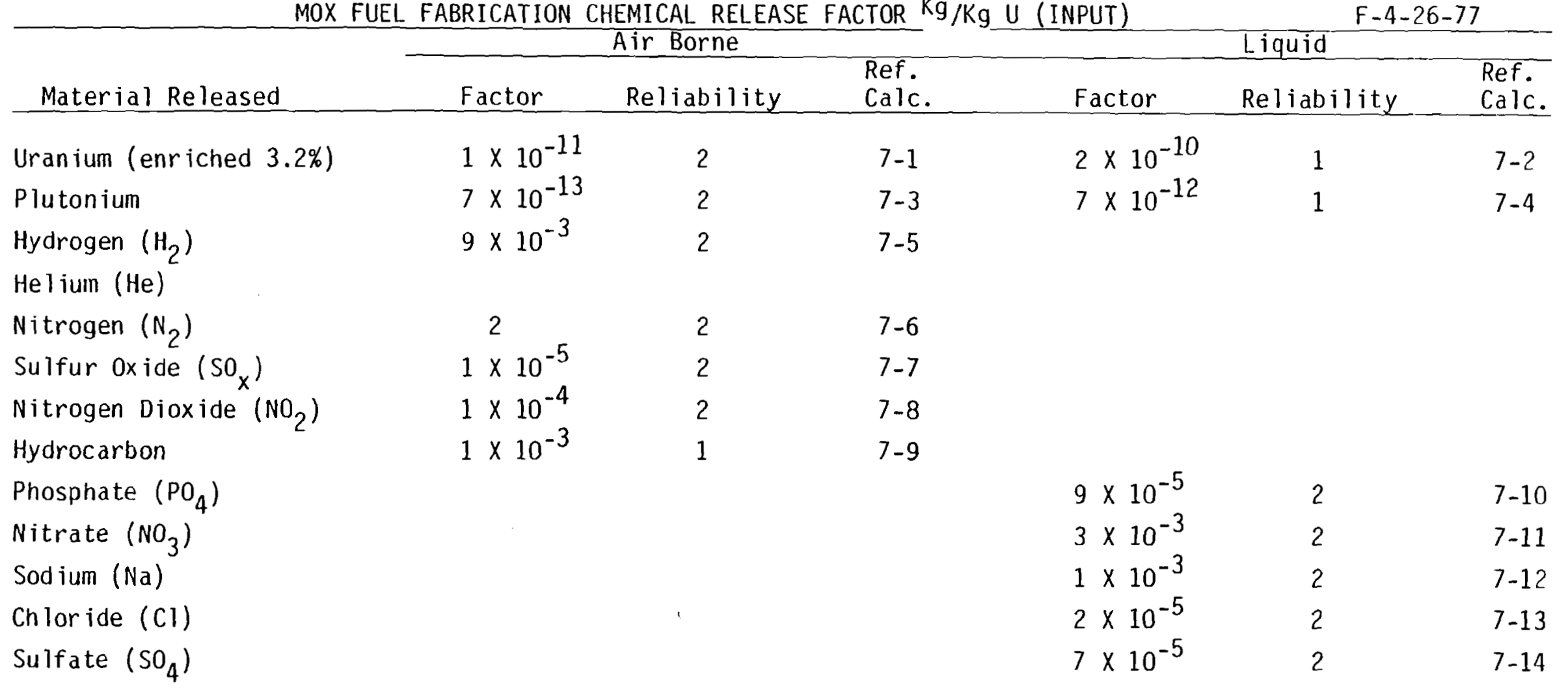


MOX FUEL FABRICATION RADIONUCLIDE RELEASE FACTOR $\mathrm{Kg} / \mathrm{Kg} U$ (INPUT)

$\mathrm{F}-4-26-77$

\begin{tabular}{|c|c|c|c|c|c|c|}
\hline \multirow[b]{2}{*}{ Material Released } & \multicolumn{3}{|c|}{ Air Borne } & \multicolumn{3}{|c|}{ Liquid } \\
\hline & Factor & Reliability & $\begin{array}{l}\text { Ref. } \\
\text { Calc. }\end{array}$ & Factor & Reliability & $\begin{array}{l}\text { Ref. } \\
\text { Calc. }\end{array}$ \\
\hline \multicolumn{7}{|c|}{ Uranium (enriched $3.2 \%$ ) } \\
\hline *Uranium - 238 & $1.42 \times 10^{-11}$ & 2 & $7-15$ & $1.52 \times 10^{-10}$ & 1 & $7-15$ \\
\hline *Uranium - 235 & $4.70 \times 10^{-13}$ & 2 & $7-15$ & $5.02 \times 10^{-12}$ & 1 & $7-15$ \\
\hline *Uranium - 234 & $2.25 \times 10^{-15}$ & 2 & $7-15$ & $2.40 \times 10^{-14}$ & 1 & $7-15$ \\
\hline \multicolumn{7}{|l|}{ Pluton ium } \\
\hline *Plutonium -242 & $6.25 \times 10^{-14}$ & 2 & $7-16$ & $6.66 \times 10^{-13}$ & 1 & $7-16$ \\
\hline *Plutonium - 241 & $1.11 \times 10^{-13}$ & 2 & $7-16$ & $1.18 \times 10^{-12}$ & 1 & $7-16$ \\
\hline$\star$ Plutonium -240 & $1.87 \times 10^{-13}$ & 2 & $7-16$ & $2.00 \times 10^{-12}$ & 1 & $7-16$ \\
\hline *Plutonium - 239 & $3.16 \times 10^{-13}$ & 2 & $7-16$ & $3.37 \times 10^{-12}$ & 1 & $7-16$ \\
\hline *Plutonium -238 & $1.74 \times 10^{-14}$ & 2 & $7-16$ & $1.85 \times 10^{-13}$ & 1 & $7-16$ \\
\hline
\end{tabular}

* Has Data Base In Computer Model 
REFERENCE CALCULATIONS: MOX FUEL FABRICATION FACILITY (365 0perating Days/yr)

4-26-77

7-1. U input to MOX fuel fabrication facility:

(See Note)

$930 \mathrm{~kg} \mathrm{U}\left(\right.$ enriched to $\left.3.2 \%{ }^{235} \mathrm{U}\right)$

day

U airborne output from the MOX fuel fabrication facility:

(See Note)

$\frac{1.37 \times 10^{-8} \mathrm{~kg} \mathrm{U} / \text { day }}{930 \mathrm{~kg} \mathrm{U} / \text { day }}=1.4710^{-11} \frac{\mathrm{kg} \mathrm{U}(\text { airborne })}{\mathrm{kg} \mathrm{U( \text {input } )}}$

$\stackrel{?}{\circ}$

(See Note)

7-2. $\frac{1.46 \times 10^{-7} \mathrm{~kg} \mathrm{U} / \text { day }}{930 \mathrm{~kg} \mathrm{U} / \mathrm{day}}=1.57 \times 10^{-10} \frac{\mathrm{kg} \mathrm{U}(1 \text { iquid) }}{\mathrm{kg} \mathrm{U(input)}}$

(See Note)

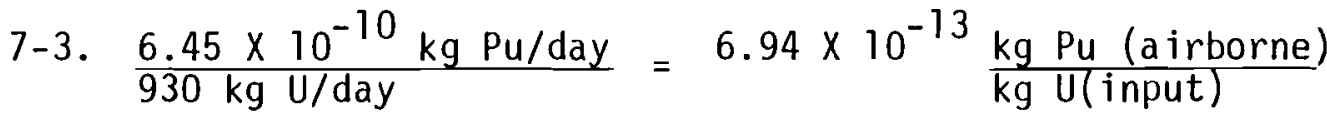

(See Note)

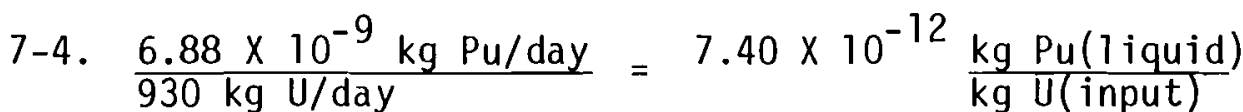




\section{REFERENCE CALCULATIONS: MOX Fuel Fabrication Facility (cont'd)}

(See Note)

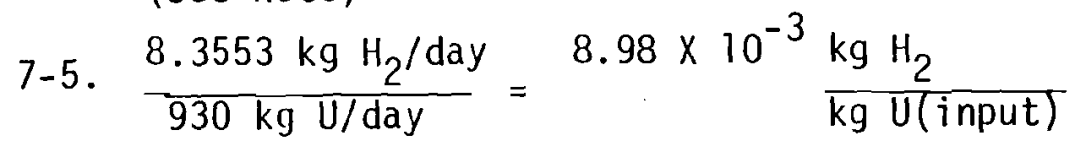

(See Note)

7-6. $\frac{1821 \mathrm{~kg} \mathrm{~N}_{2} / \text { day }}{930 \mathrm{~kg} \mathrm{U} / \text { day }}=\frac{1.96 \mathrm{~kg} \mathrm{~N}}{\mathrm{~kg} \mathrm{U} \text { (input) }}$

(See Note)

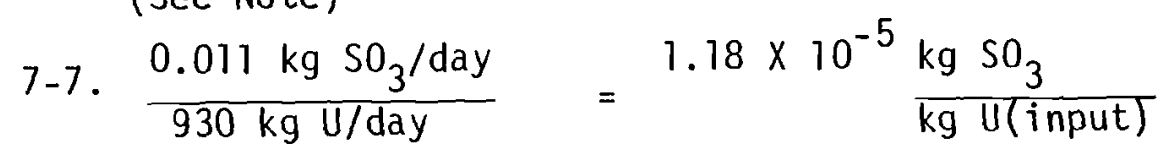

(See Note)

7-8. $\frac{0.104 \mathrm{~kg} \mathrm{NO} / \text { day }}{930 \mathrm{~kg} \mathrm{U} / \text { day }}=1.12 \times 10^{-4} \frac{\mathrm{kg} \mathrm{NO}_{2}}{\mathrm{~kg} \mathrm{U} \text { (input) }}$

7-9. Assuming all the die lubricant used in the presses is driven off in the sintering furnaces as hydrocarbons then:

400 metric $\frac{\text { tons }(U \& \mathrm{PU})}{\mathrm{yr}} \times \frac{2270 \mathrm{MOX}}{2238(\mathrm{U} \& \mathrm{PU})} \times \frac{10^{3} \mathrm{~kg}}{\text { metric ton }} \times \frac{\mathrm{yr}}{365 \text { days }} \times 0.1 \%$ die lubricant $=$ 
REFERENCE CALCULATIONS: MuX. Fuel Fabrication Facility (cont'd)

$1.24 \mathrm{~kg} \mathrm{hydrocarbons}$

day

$\therefore \frac{1.24 \mathrm{~kg} \text { hydrocarbons } / \text { day }}{930 \mathrm{~kg} \mathrm{U} / \mathrm{day}}=1.34 \times 10^{-3} \frac{\mathrm{kg} \text { hydrocarbons }}{\mathrm{kg} \mathrm{U( \text {input } )}}$

(assumed

$(21$, p. 3. 5-4)

7-10. 0.18 ibs $\mathrm{PO}_{4}^{-3}$

$\frac{0.18 \text { lbs } \mathrm{PO}_{4}^{-3}}{\text { day }} \times$

$\frac{\mathrm{kg}}{2.205 \mathrm{Tbs}}=8.16 \times 10^{-2} \mathrm{~kg} \mathrm{P0_{4 } ^ { - 3 }}$

$\therefore \frac{8.16 \times 10^{-2} \mathrm{~kg} \mathrm{PO}_{4}^{-3} / \text { day }}{930 \mathrm{~kg} \mathrm{U/ \text {day }}}=$

$8.78 \times 10^{-5} \mathrm{~kg} \mathrm{PO}_{4}^{-3}$

$\stackrel{\text { D̦ }}{\text { N }}$

Airborne $\mathrm{Pu}$ :

$\begin{array}{ll}\text { (from ref.calc. } 7-3) \\ 6.94 \times 10^{-13} \frac{\mathrm{kg} \mathrm{Pu}}{\mathrm{kg} \mathrm{U}(\text { input })} \times 0.09\end{array} \quad \begin{aligned} & \text { (see } \\ & \text { (above) }\end{aligned}=6.25 \times 10^{-14} \frac{\mathrm{kg}{ }^{242} \mathrm{Pu}}{\mathrm{kg} U(\text { input })}$

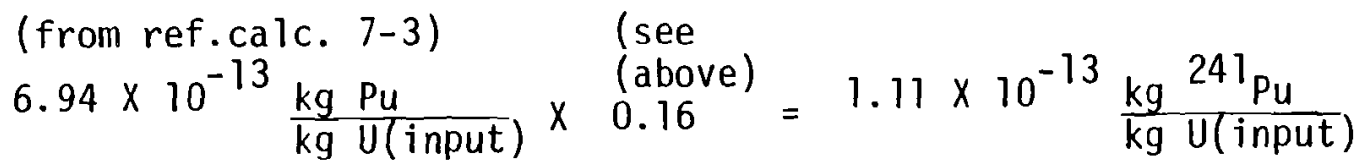




\section{REFERENCE CALCULATIONS: MOג Fuel Fabrication Facility (cont'd)}

(from ref.calc. $7-3)$
$6.94 \times 10^{-13} \frac{\mathrm{kg} \mathrm{Pu}}{\mathrm{kg} \mathrm{U}(\text { input })} \times \begin{gathered}\text { (see } \\ \text { (above) } \\ 0.27\end{gathered}=1.87 \times 10^{-13} \frac{\mathrm{kg} 240 \mathrm{Pu}}{\mathrm{kg} \mathrm{U}(\text { input })}$
(from ref.calc. 7-3)
$6.94 \times 10^{-13} \mathrm{~kg} \mathrm{Pu}$
(see $\frac{\mathrm{kg} \mathrm{Pu}}{\mathrm{kg} \mathrm{U} \text { (input) }}$
$\begin{aligned} & \text { (above) } \\ & 0.455\end{aligned}=3.16 \times 10^{-13} \mathrm{~kg}{ }^{239} \mathrm{Pu}$
$0.455=3.16 \times 10^{-15} \frac{\mathrm{kg}}{\mathrm{kg} \text { (input) }}$

$\begin{array}{ll}\text { (from ref.calc. 7-3) } \\ 6.94 \times 10^{-13} \frac{\mathrm{kg} \mathrm{Pu}}{\mathrm{kg} \mathrm{U}(\text { input })} \times \begin{array}{l}\text { (see } \\ \text { (above) }\end{array} \times 1.025\end{array}=1.74 \times 10^{-14} \frac{\mathrm{kg}{ }^{238} \mathrm{Pu}}{\mathrm{kg} \mathrm{U(input)}}$

$\stackrel{7}{\omega}$

Liquid Pu:

(from ref.calc. 7-4)

$7.40 \times 10^{-12} \mathrm{~kg} \mathrm{Pu}$

(see

$\underset{0.09}{(\text { above })}=6.66 \times 10^{-13} \frac{\mathrm{kg}{ }^{242} \mathrm{Pu}}{\mathrm{kg} U(\text { input }}$ $\mathrm{kg} \mathrm{U(input)}$

$$
\text { kg U(iriput) }
$$

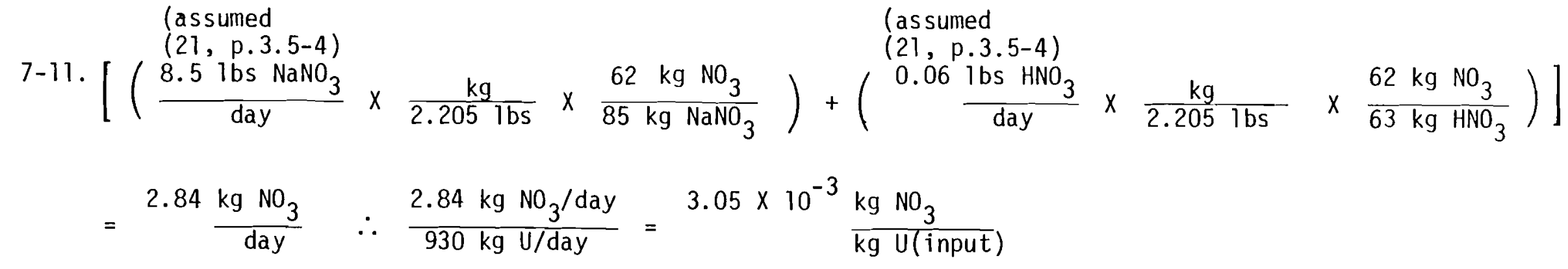


REFERENCE CALCULATIONS: MOX Fuel Fabrication Facility (cont'd)

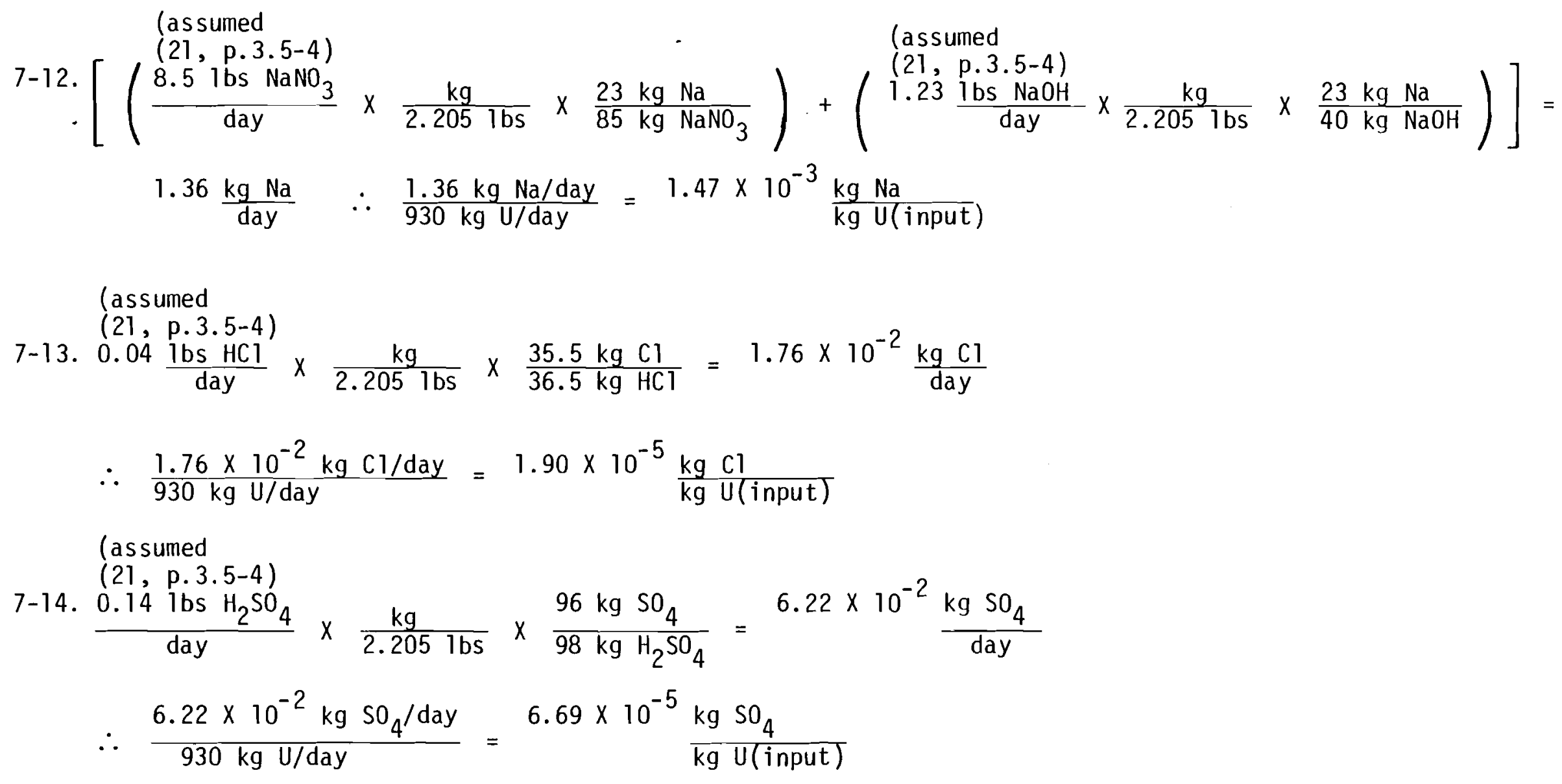


REFERENCE CALCULATIONS: MOX Fuel Fabrication Facility (cont'd)

7-15. Assuming isotopic compositions for uranium of $96.7841 \% 238 \mathrm{U}, 3.2 \%{ }^{235} \mathrm{U}$, and $0.0153 \% 234 \mathrm{U}$ as calculated in ref. calc. 6-28, 6-29 then:

Airborne U:

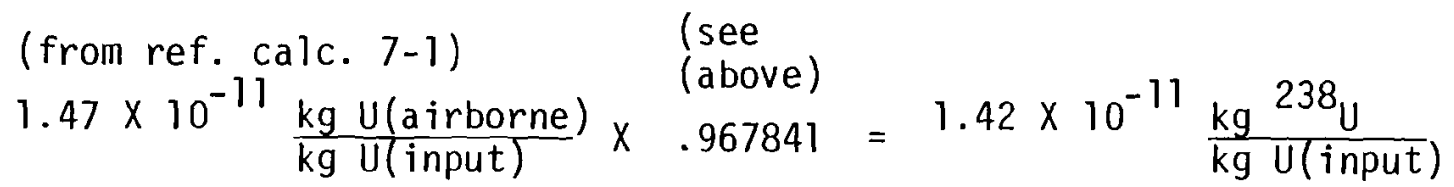

(from ref.calc. 7-1)

$1.47 \times 10^{-11} \frac{\mathrm{kg} U(\text { airborne })}{\mathrm{kg} U \text { (input) }} \times \begin{aligned} & \text { (above) } \\ & 0.032\end{aligned}=4.70 \times 10^{-13} \frac{\mathrm{kg}{ }^{235} \mathrm{U}}{\mathrm{kg} \mathrm{U}(\text { input })}$

$\underset{\sim}{\cos }$

(from ref.calc. 7-1)

$1.47 \times 10^{-11} \frac{\mathrm{kg} U \text { (airborne })}{\mathrm{kg} U(\text { input })} \times \frac{(\text { see above })}{0.000153}=2.25 \times 10^{-15} \frac{\mathrm{kg} 234 \mathrm{U}}{\mathrm{kg} U(\text { input })}$

Liquid U:

$\begin{aligned} & \text { (from ref.calc. 7-2) } \\ & 1.57 \times 10^{-10} \frac{\mathrm{kg} \mathrm{U}(1 \text { iquid) }}{\mathrm{kg} U(\text { input) }} \times .967841\end{aligned} \times 1.52 \times 10^{-10} \frac{\mathrm{kg} 238 \mathrm{U}}{\mathrm{kg} \mathrm{U(input)}}$

(from ref.calc. 7-2)

$1.57 \times 10^{-10} \frac{\mathrm{kg} U(1 \text { iquid) }}{\mathrm{kg} U(\text { input })} \times \begin{gathered}(\text { above }) \\ 0.032\end{gathered}=5.02 \times 10^{-12} \frac{\mathrm{kg}}{\mathrm{kg} U(\text { input })}$ 
REFERENCE CALCULATIONS: MOX Fuel Fabrication Facility (cont'd)

(from ref.calc. $7-2$ )

$1.57 \times 10^{-10} \frac{\mathrm{kg} \mathrm{U}(1 \text { iquid })}{\mathrm{kgU}(\text { input })} \times 0.000153=2.40 \times 10^{-14} \frac{\mathrm{kg} 234 \mathrm{U}}{\mathrm{kg} \mathrm{U} \text { (input) }}$

7-16. Assuming isotopic compositions for plutonium of $9 \%{ }^{242} \mathrm{Pu}, 16 \%{ }^{241} \mathrm{Pu}, 27 \%{ }^{240} \mathrm{Pu}, 45.5 \%{ }^{239} \mathrm{Pu}$, $2.5 \%{ }^{238} \mathrm{Pu}$ (See Note)

$7.40 \times 10^{-12} \frac{\mathrm{kg} \mathrm{Pu}}{\mathrm{kgU}(\text { input })} \times \begin{gathered}(\text { above }) \\ 0.16\end{gathered}=1.18 \times 10^{-12} \frac{\mathrm{kg} 241 \mathrm{Pu}}{\mathrm{kg} U(\text { input })}$

(from ref.calc. 7-4)

$7.40 \times 10^{-12} \mathrm{~kg} \mathrm{Pu}$ $\frac{\mathrm{kg} \mathrm{Pu}}{\mathrm{kg} U(\text { input })}$

\section{(see}

(above) $\quad 2.00 \times 10^{-12} \mathrm{~kg}^{240} \mathrm{Pu}$

$\times \quad 0.27$

kg U(input)

(from ref.calc. 7-4)

$7.40 \times 10^{-12} \mathrm{~kg} \mathrm{Pu}$ $\frac{\mathrm{kg} U(\text { input })}{\mathrm{kg}}$

\section{(see}

$\begin{aligned} & \text { (above) } \\ & 0.455\end{aligned}=3.37 \times 10^{-12} \frac{\mathrm{kg}^{239} \mathrm{Pu}}{\mathrm{kg} \mathrm{U}(\text { input })}$

(from ref.calc. 7-4)

$7.40 \times 10^{-12}$

$\frac{\mathrm{kg} \mathrm{Pu}}{\mathrm{kg} U(\text { input })}$

(see
$\left(\begin{array}{l}\text { above }) \\ 0.025\end{array}=1.85 \times 10^{-13} \frac{\mathrm{kg}{ }^{238} \mathrm{Pu}}{\mathrm{kg} U(\text { input })}\right.$

NOTE: Many of the references used in this section were obtained through use of the references and other extensively footnoted data contained in the 800 pages of: "Description and Analysis of Reference Fuel Fabrication Facilities for $\mathrm{LWR}_{2} \mathrm{UO}_{2}$ and $\mathrm{UO}_{2}-\mathrm{PuO}_{2}$ Fuels" prepared by Wendell $\mathrm{J}$. Bailley, Battelle Pacific Northwest Laboratories. Unfortunately, it has not as yet been released for publication, and therefore cannot be used as a direct reference. 
PWR REACTOR CHEMICAL RELEASE FACTOR $\mathrm{Kg} / \mathrm{Kg} U$ (INPUT)

\begin{tabular}{|c|c|c|c|c|c|c|}
\hline \multirow[b]{2}{*}{ Material Released } & \multicolumn{3}{|c|}{ Air Borne } & \multicolumn{3}{|c|}{ Liquid } \\
\hline & Factor & Reliability & $\begin{array}{l}\text { Ref. } \\
\text { Calc. }\end{array}$ & Factor & Reliability & $\begin{array}{l}\text { Ref. } \\
\text { Calc. }\end{array}$ \\
\hline Argon & $2.0 \times 10^{-14}$ & 2 & $8-2$ & & & \\
\hline Krypton & $5.9 \times 10^{-8}$ & 2 & $8-51$ & & & \\
\hline Xenon & $7.8 \times 10^{-10}$ & 2 & $8-52$ & & & \\
\hline Rubidium & $1.9 \times 10^{-17}$ & 2 & $8-53$ & $1.6 \times 10^{-16}$ & 2 & $8-69$ \\
\hline Cesium & $7.0 \times 10^{-13}$ & 2 & $8-54$ & $4.3 \times 10^{-11}$ & 2 & $8-70$ \\
\hline Bromine & $6.2 \times 10^{-19}$ & 2 & $8-55$ & $2.4 \times 10^{-18}$ & 2 & $8-67$ \\
\hline Iodine & $5.4 \times 10^{-15}$ & 2 & $8-56$ & $3.3 \times 10^{-13}$ & 2 & $8-68$ \\
\hline Chromium & $1.5 \times 10^{-16}$ & 2 & $8-9$ & $3.3 \times 10^{-15}$ & 2 & $8-32$ \\
\hline Manganese & $1.8 \times 10^{-15}$ & 2 & $8-10$ & $8.9 \times 10^{-15}$ & 2 & $8-33$ \\
\hline Iron & $4.7 \times 10^{-15}$ & 2 & $8-57$ & $1.1 \times 10^{-13}$ & 2 & $8-71$ \\
\hline Cobalt & $2.3 \times 10^{-14}$ & 2 & $8-58$ & $5.3 \times 10^{-13}$ & 2 & $8-72$ \\
\hline Stront ium & $1.3 \times 10^{-15}$ & 2 & $8-59$ & $1.3 \times 10^{-14}$ & 2 & $8-73$ \\
\hline Yttrium & $5.8 \times 10^{-16}$ & 2 & $8-60$ & $1.3 \times 10^{-14}$ & 2 & $8-74$ \\
\hline Zirconium & $2.2 \times 10^{-17}$ & 2 & $8-15$ & $1.8 \times 10^{-15}$ & 2 & $8-38$ \\
\hline Niobium & $9.9 \times 10^{-18}$ & 2 & $8-16$ & $1.2 \times 10^{-15}$ & 2 & $8-39$ \\
\hline Molybdenum & $6.0 \times 10^{-15}$ & 2 & $8-17$ & $1.1 \times 10^{-13}$ & 2 & $8-40$ \\
\hline Technetium & $4.8 \times 10^{-16}$ & 2 & $8-18$ & & & \\
\hline Ruthen ium & $3.0 \times 10^{-17}$ & 2 & $8-61$ & $7.8 \times 10^{-16}$ & 2 & $8-75$ \\
\hline Rhodium & $1.2 \times 10^{-20}$ & 2 & $8-62$ & $2.3 \times 10^{-19}$ & 2 & $8-76$ \\
\hline Silver & & & & $1.8 \times 10^{-15}$ & 2 & $8-43$ \\
\hline Tellurium & $1.1 \times 10^{-15}$ & 2 & $8-63$ & $2.4 \times 10^{-14}$ & 2 & $8-77$ \\
\hline Barium & $2.2 \times 10^{-17}$ & 2 & $8-64$ & $4.6 \times 10^{-16}$ & 2 & $8-78$ \\
\hline
\end{tabular}


PWR REACTOR (cont'd) CHEMICAL RELEASE FACTOR $\mathrm{Kg} / \mathrm{Kg} \mathrm{U}$ (INPUT)

\begin{tabular}{|c|c|c|c|c|c|c|}
\hline \multirow[b]{2}{*}{ Material Released } & \multicolumn{3}{|c|}{ Air Borne } & \multicolumn{3}{|c|}{ Liquid } \\
\hline & Factor & Reliability & $\begin{array}{l}\text { Ref. } \\
\text { Calc. }\end{array}$ & Factor & Reliability & $\begin{array}{l}\text { Ref. } \\
\text { Calc. }\end{array}$ \\
\hline Lanthanum & $2.0 \times 10^{-18}$ & 2 & $8-23$ & $5.2 \times 10^{-17}$ & 2 & $8-46$ \\
\hline Cerium & $9.4 \times 10^{-17}$ & 2 & $8-65$ & $3.2 \times 10^{-14}$ & 2 & $8-79$ \\
\hline Praseodymium & $5.2 \times 10^{-18}$ & 2 & $8-66$ & $1.2 \times 10^{-16}$ & 2 & $8-80$ \\
\hline Neptun ium & $3.5 \times 10^{-17}$ & 2 & $8-26$ & $5.8 \times 10^{-16}$ & 2 & $8-49$ \\
\hline Tritium & $1.0 \times 10^{-9}$ & 2 & $8-27$ & $3.5 \times 10^{-9}$ & 2 & $8-50$ \\
\hline Sulfate $\left(\mathrm{SO}_{4}\right)$ & & & & $7 \times 10^{1}$ & 2 & $8-81$ \\
\hline Volatile Amines & & & & $9 \times 10^{-4}$ & 2 & $8-82$ \\
\hline Boron (B) & & & & $6 \times 10^{-3}$ & 2 & $8-83$ \\
\hline Sodium ( $\mathrm{Na})$ & & & & 7 & 2 & $8-84$ \\
\hline Sulfite $\left(\mathrm{SO}_{3}\right)$ & & & & $7 \times 10^{-5}$ & 2 & $8-85$ \\
\hline Orthophosphate & & & & $2 \times 10^{-2}$ & 2 & $8-87$ \\
\hline \multicolumn{7}{|l|}{ Alum } \\
\hline Sulfuric Acid $\left(\mathrm{H}_{2} \mathrm{SO}_{4}\right)$ & $x$ & & & & & \\
\hline Chlor ine $\left(\mathrm{Cl}_{2}\right)$ & $x$ & & & $x$ & & \\
\hline Carbon Monoxide ( $\mathrm{CO}$ ) & $x$ & & & & & \\
\hline Nitrogen Oxide $\left(\mathrm{NO}_{x}\right)$ & $x$ & & & & & \\
\hline Sulfur oxide $\left(\mathrm{SO}_{\mathrm{x}}\right)$ & $x$ & & & & & \\
\hline Aldehyde & $x$ & & & & & \\
\hline \multicolumn{7}{|l|}{ Nonradioactive } \\
\hline Particulate & $x$ & & & & & \\
\hline
\end{tabular}


PWR REACTOR (cont'd) CHEMICAL RELEASE FACTOR $\mathrm{Kg} / \mathrm{Kg} U$ (INPUT)

\begin{tabular}{|c|c|c|c|c|c|c|}
\hline \multirow[b]{2}{*}{ Material Released } & \multicolumn{3}{|c|}{ Air Borne } & \multicolumn{3}{|c|}{ Liquid } \\
\hline & Factor & Reliability & $\begin{array}{l}\text { Ref. } \\
\text { Calc. }\end{array}$ & Factor & Reliability & $\begin{array}{l}\text { Ref. } \\
\text { Calc. }\end{array}$ \\
\hline Hydrocarbon & $x$ & & & & & \\
\hline Chloride & & & & $x$ & & \\
\hline 0 il and Grease & & & & $x$ & & \\
\hline
\end{tabular}




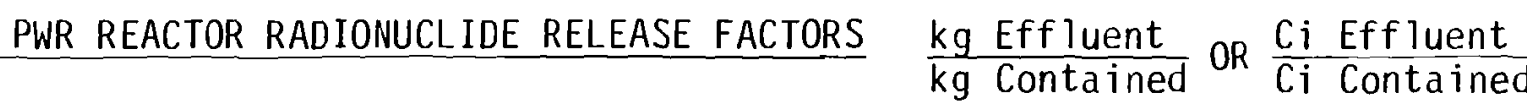

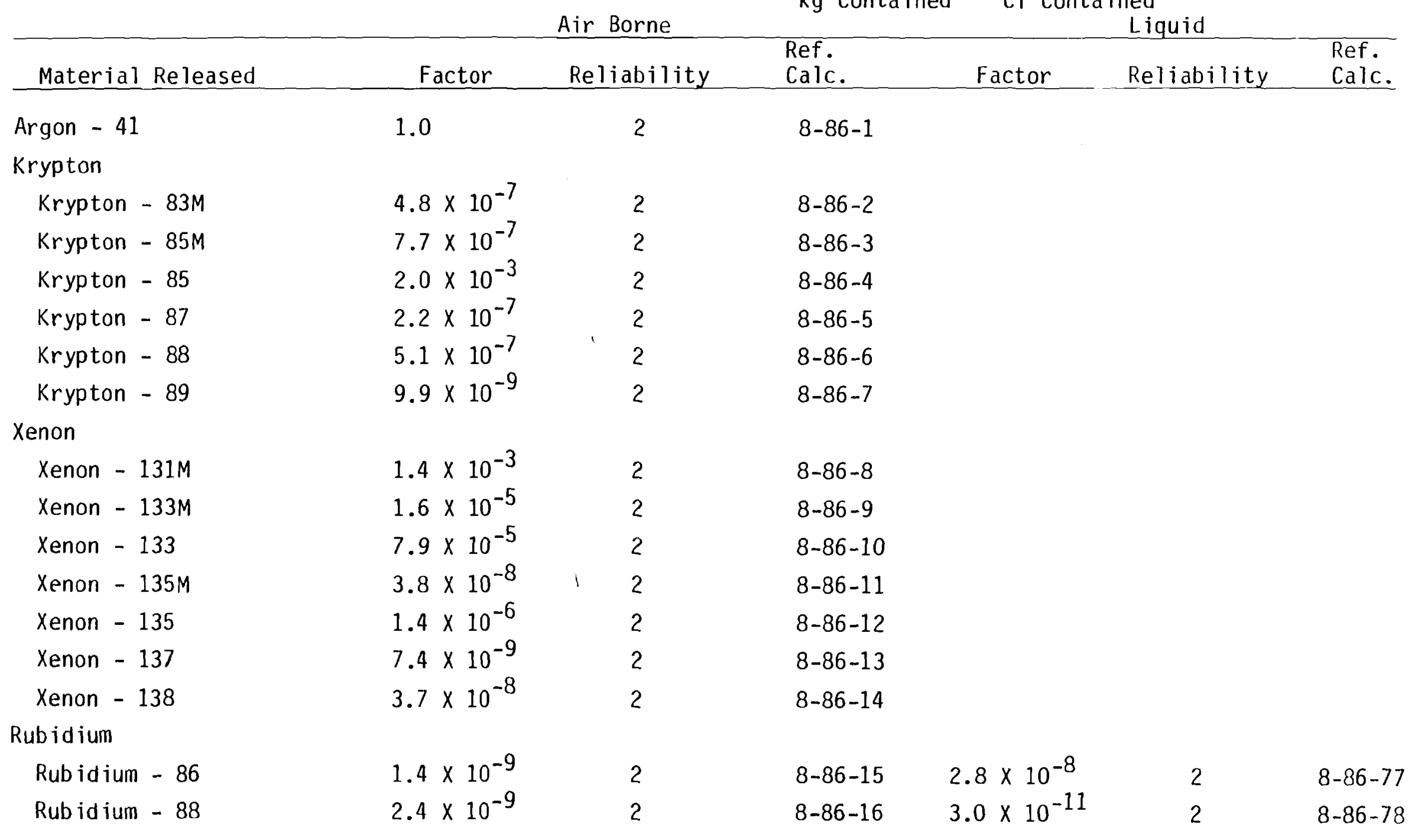


Air Borne

Material Released

Cesium

$$
\begin{aligned}
& \text { Cesium - } 134 \\
& \text { Cesium - } 136 \\
& \text { Cesium - } 137
\end{aligned}
$$

Bromine

$$
\begin{aligned}
& \text { Bromine }-83 \\
& \text { Bromine }-84 \\
& \text { Bromine - } 85
\end{aligned}
$$

\section{Iodine}

$\stackrel{\infty}{\infty}$

Iodine -130
Iodine -131
Iodine -132
Iodine -133
Iodine -134
Iodine -135
Chromium - 51
Manganese - 54
Iron
Irón - 55
Iron - 59

$\begin{array}{ll}2.2 \times 10^{-10} & 2 \\ 1.7 \times 10^{-9} & 2 \\ 5.9 \times 10^{-10} & 2\end{array}$

$1.5 \times 10^{-10}$

$3.3 \times 10^{-12}$

$1.2 \times 10^{-10}$

$6.9 \times 10^{-10}$

$1.6 \times 10^{-10}$

$4.2 \times 10^{-10}$

$4.6 \times 10^{-11}$

$2.5 \times 10^{-10}$

$5.6 \times 10^{-8}$

$1.8 \times 10^{-6}$

$2.7 \times 10^{-7}$

$1.8 \times 10^{-6}$

\section{2}

2

2

2
$3.0 \times 10^{-11}$

$\begin{array}{ll}8-86-17 & 1.8 \times 10^{-8} \\ 8-86-18 & 3.5 \times 10^{-8} \\ 8-86-19 & 3.2 \times 10^{-8}\end{array}$
Liquid Ref. Calc. Factor Reliability

$\begin{array}{ll}8-86-20 & 6.4 \times 10^{-10} \\ 8-86-21 & 8.4 \times 10^{-12} \\ 8-86-22 & 5.2 \times 10^{-15}\end{array}$

$2.9 \times 10^{-9}$

$8-86-23$

$4.4 \times 10^{-8}$

8-86-24

$3.4 \times 10^{-9}$

8-86-25

$1.7 \times 10^{-8}$

8-86-26

$3.7 \times 10^{-11}$

8-86-27

$3.4 \times 10^{-9}$

$8-86-28$

$1.2 \times 10^{-6}$

8-86-29

$8.7 \times 10^{-6}$

$8-86-3 i$

$6.2 \times 10^{-6}$

$8-86-32$

$4.8 \times 10^{-5}$ Reliability

Ref. Calc.

Reliability

-

$8-86-79$
$8-86-80$
$8-86-81$

$8-86-68$
$8-86-69$
$8-86-70$

$8-86-71$

$8-86-72$

$8-86-73$

$8-86-74$

$8-86-75$

$8-86-76$

$8-86-82$

8-86-83

8-86-84

8-86-85 


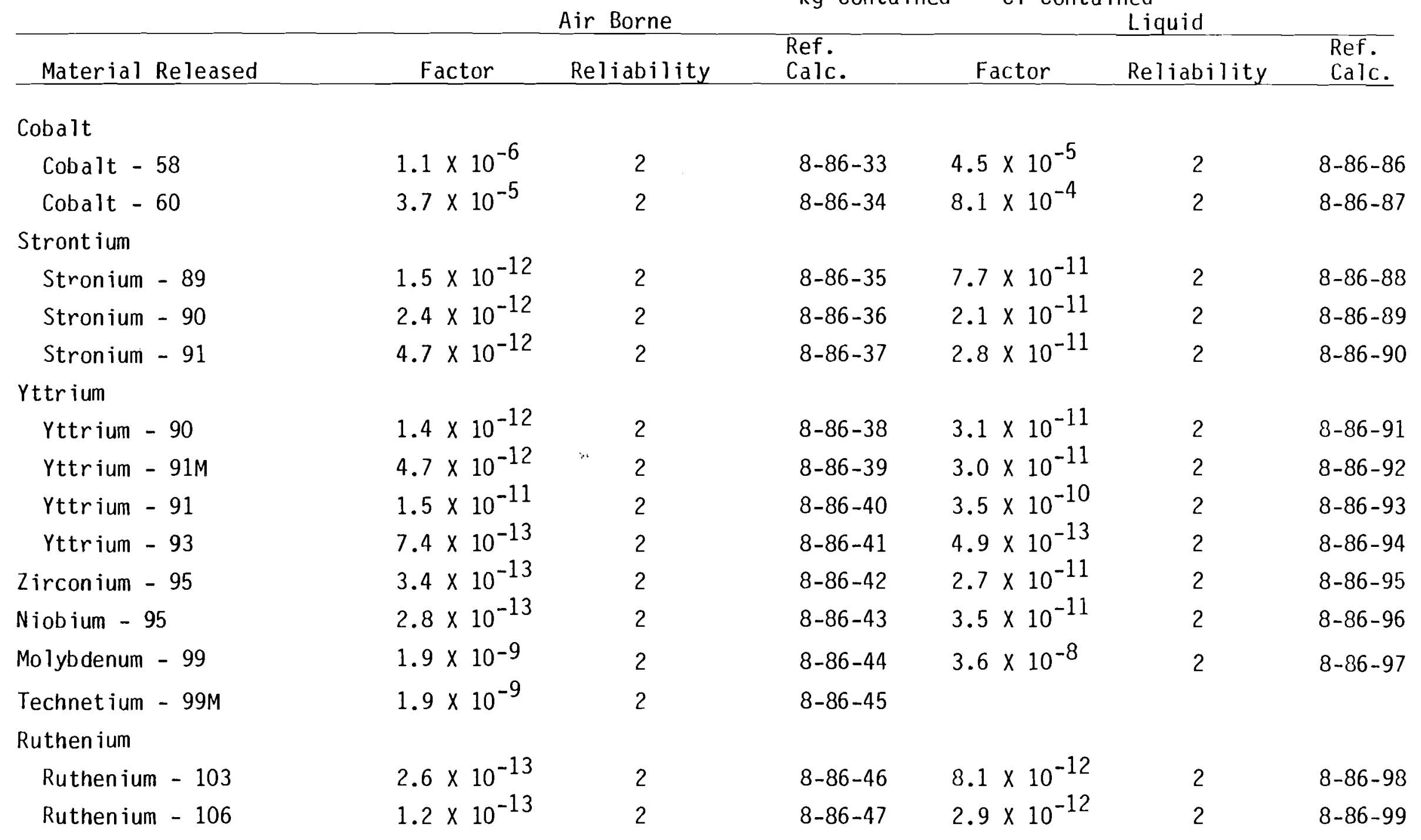




\begin{tabular}{|c|c|c|c|c|c|c|}
\hline & & Air Borne & & & Liquid & \\
\hline Material Released & Factor & Reliability & $\begin{array}{l}\text { Ref. } \\
\text { Calc. }\end{array}$ & Factor & Reliability & $\begin{array}{l}\text { Ref. } \\
\text { Calc. }\end{array}$ \\
\hline \multicolumn{7}{|l|}{ Rhodium } \\
\hline Rhodium - 103M & $2.6 \times 10^{-13}$ & 2 & $8-86-48$ & $5.9 \times 10^{-12}$ & 2 & $8-86-100$ \\
\hline Rhodium - 106 & $1.0 \times 10^{-11}$ & 2 & $8-86-49$ & $6.0 \times 10^{-11}$ & 2 & $8-86-101$ \\
\hline Silver - 110M & & & & $2.3 \times 10^{-9}$ & 2 & $8-86-102$ \\
\hline \multicolumn{7}{|l|}{ Tellurium } \\
\hline Tellurium - 125M & $6.0 \times 10^{-11}$ & 2 & $8-86-50$ & $1.5 \times 10^{-9}$ & 2 & $8-86-103$ \\
\hline Tellurium - 127M & $1.2 \times 10^{-10}$ & 2 & $8-86-51$ & $3.1 \times 10^{-9}$ & 2 & $8-86-104$ \\
\hline Tellurium - 127 & $8.1 \times 10^{-11}$ & 2 & $8-86-52$ & $9.7 \times 10^{-10}$ & 2 & $8-86-105$ \\
\hline Tellurium - 129M & $1.6 \times 10^{-10}$ & 2 & $8-86-53$ & $3.9 \times 10^{-9}$ & 2 & $8-86-106$ \\
\hline Tellurium - 129 & $3.2 \times 10^{-11}$ & 2 & $8-86-54$ & $4.3 \times 10^{-10}$ & 2 & $8-86-107$ \\
\hline Tellurium - 131M & $1.4 \times 10^{-10}$ & 2 & $8-86-55$ & $1.7 \times 10^{-9}$ & 2 & $8-86-108$ \\
\hline Tellurium - 131 & $9.6 \times 10^{-12}$ & 2 & $8-86-56$ & $5.5 \times 10^{-11}$ & 2 & $8-86-109$ \\
\hline Tellurium - 132 & $1.6 \times 10^{-10}$ & 2 & $8-86-57$ & $2.9 \times 10^{-9}$ & 2 & $8-86-110$ \\
\hline \multicolumn{7}{|l|}{ Barium } \\
\hline Barium - 137M & $5.2 \times 10^{-15}$ & 2 & $8-86-58$ & $1.6 \times 10^{-17}$ & 2 & $8-86-111$ \\
\hline Barium - 140 & $1.1 \times 10^{-12}$ & 2 & $8-86-59$ & $2.4 \times 10^{-11}$ & 2 & $8-86-112$ \\
\hline Lanthanum - 140 & $7.4 \times 10^{-13}$ & 2 & $8-86-60$ & $2.0 \times 10^{-11}$ & 2 & $8-86-113$ \\
\hline
\end{tabular}


$\mathrm{kg}$ Effluent OR Ci Effluent

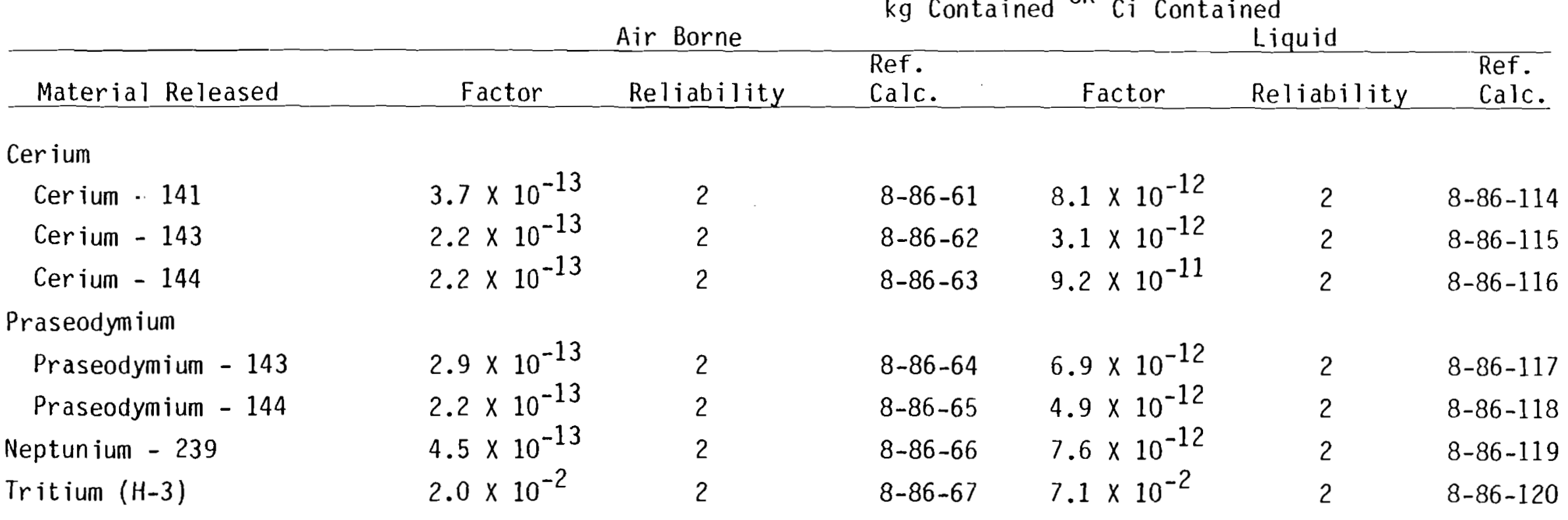

$\stackrel{\infty}{\infty}$ Carbon -14 


\section{REFERENCE CALCULATIONS: PWR REACTOR (290 Operating Days/year)}

$10-1-78$

8-1. U input to the PWR reactor assuming $1 / 3$ of the fuel is changed out every year:

$$
\begin{aligned}
& \text { (22, p. 11-2) } \\
& 101,033 \mathrm{~kg} \mathrm{U0} 2
\end{aligned} \times \frac{238 \mathrm{U}}{270 \mathrm{U0}_{2}} \times \frac{(\mathrm{l} / 3}{1 \text { year }} \times \frac{\text { year }}{290 \text { operating days }}=102 \frac{\mathrm{kg} \mathrm{U}}{\text { day }}
$$

Reference calculations through use the following format for calculation of numbers:

$$
\frac{\text { A Ci radionuclide }}{\text { year }} \times \frac{\text { year }}{290 \text { op. days }} \times \frac{\text { gradionuclide }}{B \text { Ci radionuclide }} \times \frac{\mathrm{kg}}{10^{3} \mathrm{~g}}=\frac{\mathrm{C} \mathrm{kg} \text { radionuclide }}{\text { operating days }}
$$

Note: Al1 A values in reference calculations 8-2 through 8-27 are from reference 32, table 11.3-3; all $A$ values in reference calculations 8-28 through 8-50 are from reference 32, table $11.2-12$. Al1 $B$ values in reference calculations 8-51 through 8-87 are from reference 24 .

8-2. Argon 41:
$A=2.5 \times 10^{1}$
$B=4.18 \times 10^{7}$
$C=2.06 \times 10^{-12}$
$D=2.02 \times 10^{-14}$

8-3. Krypton 83M:
$A=8.26 \times 10^{-1}$
$B=\stackrel{(\# 1)}{2.03} \times 10^{7}$
$C=1.40 \times 10^{-13}$
$D=1.38 \times 10^{-15}$

Krypton 85M:
$A=4.17$
$B=\begin{aligned} & (\# 2) \\ & 8.40 \times 10^{6}\end{aligned}$
$c=1.71 \times 10^{-12}$
$D=1.68 \times 10^{-14}$

Krypton $85:$
$A=6.80 \times 10^{2}$
$B=3.91 \times 10^{2}$
$C=6.00 \times 10^{-6}$
$D=5.88 \times 10^{-8}$ 
REFERENCE CALCULATIONS: PWR Reactor (cont'd)

Krypton 87 :
$A=2.29$
$B=\frac{(\# 4)}{2.84} \times 10^{7}$
$C=2.78 \times 10^{-13}$
$D=2.73 \times 10^{-15}$

Krypton 88:

$A=7.81$

$B=\frac{(\# 5)}{1.27 \times 10^{7}}$

$C=2.12 \times 10^{-12}$

$D=2.08 \times 10^{-14}$

Krypton 89:
$A=1.89 \times 10^{-1}$
$B=\frac{(\# 6)}{6.60 \times 10^{8}}$
$C=9.87 \times 10^{-16}$
$D=9.68 \times 10^{-18}$

8-4. Xenon 131M:
$A=2.89 \times 10^{2}$
$B=\stackrel{(\# 7)}{8.45} \times 10^{4}$
$C=1.18 \times 10^{-8}$
$D=1.16 \times 10^{-10}$

$\underset{\substack{1 \\ \infty}}{\infty}$

Xenon 133M:
$A=1.76 \times 10^{1}$
$B=\stackrel{(\# 8)}{4.35 \times 10^{5}}$
$C=1.40 \times 10^{-10}$
$D=1.37 \times 10^{-12}$

Xenon 133:
$A=3.66 \times 10^{3}$
$B=1.86 \times 10^{5}$
$c=6.79 \times 10^{-8}$
$D=6.65 \times 10^{-10}$

Xenon 135M:
$A=4.73 \times 10^{-1}$
$B=\frac{(\# 10)}{8.93 \times 10^{7}}$
$C=1.83 \times 10^{-14}$
$D=1.79 \times 10^{-16}$

Xenon 135:
$A=1.56 \times 10^{1}$
$B=2.52 \times 10^{6}$
$c=2.13 \times 10^{-11}$
$D=2.09 \times 10^{-13}$

Xenon 137:
$A=3.36 \times 10^{-1}$
$B=3.52 \times 10^{8}$
$c=3.29 \times 10^{-15}$
$D=3.23 \times 10^{-17}$ 


\section{REFERENCE CALCULATIONS: PWR Reactor (cont'd)}

Xenon 138:
$A=1.61$
$B=\frac{(\# 13)}{8.02 \times 10^{7}}$
$C=6.92 \times 10^{-14}$
$D=6.79 \times 10^{-16}$

8-5. Rubidium 86:
$A=1.96 \times 10^{-5}$
$B=\stackrel{(\# 19)}{8.14} \times 10^{4}$
$C=8.30 \times 10^{-16}$
$D=8.14 \times 10^{-18}$

Rubidium 88:
$A=3.73 \times 10^{-2}$
$B=1.20 \times 10^{8}$
$c=1.07 \times 10^{-15}$
$D=1.05 \times 10^{-17}$

8-6. Cesium 134:
$A=1.62 \times 10^{-3}$
$B=(\# 51) \times 10^{3}$
$C=4.30 \times 10^{-12}$
$D=4.21 \times 10^{-14}$

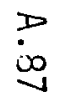
Cesium 136:
$A=2.95 \times 10^{-3}$
$B=7^{(\# 52)} \times 10^{4}$
$c=1.38 \times 10^{-13}$
$D=1.35 \times 10^{-15}$

Cesium 137:
$A=1.67 \times 10^{-3}$
$B=8^{(\# 53)} 8.64 \times 10^{1}$
$c=6.67 \times 10^{-11}$
$D=6.53 \times 10^{-13}$

8-7. Bromine 83:
$A=2.56 \times 10^{-4}$
$B=l^{(\# 84)} \times 1.57 \times 10^{7}$
$c=5.62 \times 10^{-17}$
$D=5.51 \times 10^{-19}$
Bromine 84:
$A=1.33 \times 10^{-4}$
$B=7^{(\# 85)} \times 10^{7}$
$c=6.51 \times 10^{-18}$
$D=6.39 \times 10^{-20}$ 
REFERENCE CALCULATIONS: PWR Reactor (cont'd)

Bromine 85:
$A=1.58 \times 10^{-5}$
$B=\frac{(\# 99)}{7.37} \times 10^{8}$
$C=7.39 \times 10^{-20}$
$D=7.25 \times 10^{-22}$

8-8. Iodine 130:
$A=1.16 \times 10^{-4}$
$B=1.96 \times 10^{6}$
$C=2.04 \times 10^{-16}$
$D=2.00 \times 10^{-18}$

Iodine 131:
$A=1.72 \times 10^{-2}$
$B=\stackrel{(\# 15)}{1.24} \times 10^{5}$
$C=4.78 \times 10^{-13}$
$D=4.69 \times 10^{-15}$

Iodine 132:
$A=5.59 \times 10^{-3}$
$B=l^{(\# 16)} \times 10^{7}$
$C=1.87 \times 10^{-15}$
$D=1.83 \times 10^{-17}$

Iodine 133:
$A=1.94 \times 10^{-2}$
$B=\frac{(\# 17)}{1.13 \times 10^{6}}$
$C=5.92 \times 10^{-14}$
$D=5.80 \times 10^{-16}$

Iodine 134:
$A=2.44 \times 10^{-3}$
$B=2.70 \times 10^{7}$
$c=3.12 \times 10^{-16}$
$D=3.06 \times 10^{-18}$

Iodine 135:
$A=1.03 \times 10^{-2}$
$B=3.47 \times 10^{6}$
$C=1.02 \times 10^{-14}$
$D=1.00 \times 10^{-16}$

8-9. Chromium 51:
$A=4.18 \times 10^{-4}$
$B=\stackrel{(\# 64)}{9.21 \times 10}$
$C=1.57 \times 10^{-14}$
$D=1.53 \times 10^{-16}$

8-10. Manganese 54:
$A=4.24 \times 10^{-4}$
$B=7.98 \times 10^{3}$
$c=1.83 \times 10^{-13}$
$D=1.80 \times 10^{-15}$ 
REFERENCE CALCULATIONS: PWR Reactor (cont'd)

8-11. Iron 55:
$A=3.35 \times 10^{-4}$
$B=2.50 \times 10^{3}$
$C=4.62 \times 10^{-13}$
$D=4.53 \times 10^{-15}$

Iron 59:
$A=1.77 \times 10^{-4}$
$B=4.91 \times 10^{4}$
$C=1.24 \times 10^{-14}$
$D=1.22 \times 10^{-16}$

8-12. Cobalt 58:
$A=1.91 \times 10^{-3}$
$B=3.16 \times 10^{4}$
$C=2.08 \times 10^{-13}$
$D=2.04 \times 10^{-15}$

Cobalt 60:
$A=6.86 \times 10^{-4}$
$B=1.13 \times 10^{3}$
$C=2.09 \times 10^{-12}$
$D=2.05 \times 10^{-14}$

8-13. Strontium 89:
$A=3.21 \times 10^{-5}$
$B=2.82 \times 10^{4}$
$C=3.93 \times 10^{-15}$
$D=3.85 \times 10^{-17}$

Strontium 90:
$A=5.47 \times 10^{-6}$
$B={ }^{(\# 22)} 1.42 \times 10^{2}$
$c=1.33 \times 10^{-13}$
$D=1.30 \times 10^{-15}$

Strontium 91 :
$A=1.23 \times 10^{-4}$
$B=\stackrel{(\# 23)}{3.56 \times 10^{6}}$
$C=1.19 \times 10^{-16}$
$D=1.17 \times 10^{-18}$

8-14. Yttrium 90:
$A=3.35 \times 10^{6}$
$B=\frac{(\# 24)}{5.44} \times 10^{5}$
$C=2.12 \times 10^{17}$
$D=2.08 \times 10^{-19}$

Yttrium 914:

$\mathrm{A}=7.25 \times 10^{-5}$

$B=4.13)$

$c=6.05 \times 10^{-18}$

$D=5.93 \times 10^{-20}$

Yttrium 91:

$\mathrm{A}=4.18 \times 10^{-4}$

$B=2.44 \times 10^{4}$

$C=5.91 \times 10^{-14}$

$D=5.79 \times 10^{-16}$ 
REFERENCE CALCULATIONS: PWR Reactor (cont'd)

Yttrium 93:
$A=2.42 \times 10^{-5}$
$B=3.31 \times 10^{6}$
$C=2.52 \times 10^{-17}$
$D=2.47 \times 10^{-19}$

8-15. Zirconium 95:
$A=1.36 \times 10^{-5}$
$B=2.12 \times 10^{4}$
$C=2.21 \times 10^{-15}$
$D=2.17 \times 10^{-17}$

8-16. Niobium 95:
$A=1.15 \times 10^{-5}$
$B=3^{(\# 30)} \times 10^{4}$
$C=1.01 \times 10^{-15}$
$D=9.94 \times 10^{-18}$

8-17. Mol ybdenum 99:
$A=8.46 \times 10^{-2}$
$B=\frac{(\# 33)}{4.75} \times 10^{5}$
$C=6.14 \times 10^{-13}$
$D=6.02 \times 10^{-15}$

8-18. Technetium 99M:
$A=7.46 \times 10^{-2}$
$B=5.28 \times 10^{6}$
$C=4.87 \times 10^{-14}$
$D=4.78 \times 10^{-16}$

8-19. Ruthenium 103:
$A=9.17 \times 10^{-6}$
$B=3.20 \times 10^{4}$
$c=9.88 \times 10^{-16}$
$D=9.69 \times 10^{-18}$

Ruthenium 106:
$A=1.97 \times 10^{-6}$
$B=3.36 \times 10^{3}$
$c=2.02 \times 10^{-15}$
$D=1.98 \times 10^{-17}$

8-20. Rhodium 103M:
$A=9.12 \times 10^{-6}$
$B=\frac{(\# 37)}{3.20} \times 10^{7}$
$c=9.83 \times 10^{-19}$
$D=9.63 \times 10^{-21}$

Rhodium 106:
$A=2.26 \times 10^{-4}$
$B=\frac{(\# 39)}{3.55 \times 10^{9}}$
$C=2.20 \times 10^{-19}$
$D=2.15 \times 10^{-21}$ 
REFERENCE CALCULATIONS: PWR Reactor (cont'd)

8-21. Tellurium 125M:
$A=5.62 \times 10^{-6}$
$B=1.80 \times 10^{4}$
$c=1.08 \times 10^{-15}$
$D=1.06 \times 10^{-17}$

Tellurium 127M:
$A=5.56 \times 10^{-5}$
$B=\stackrel{(\# 44)}{9.44} \times 10^{3}$
$C=2.03 \times 10^{-14}$
$D=1.99 \times 10^{-16}$

Telluriun 127:
$A=1.67 \times 10^{-4}$
$B=\stackrel{(\# 45)}{2.63} \times 10^{6}$
$C=2.19 \times 10^{-16}$
$D=2.15 \times 10^{-18}$

Tellurium 129M:
$A=2.70 \times 10^{-4}$
$B=2.98 \times 10^{4}$
$c=3.12 \times 10^{-14}$
$D=3.06 \times 10^{-16}$

Tellurium 129:
$A=3.13 \times 10^{-4}$
$B=2.11 \times 10^{7}$
$c=5.12 \times 10^{-17}$
$D=5.01 \times 10^{-19}$

Tellurium 131M:
$A=5.02 \times 10^{-4}$
$B=7.98 \times 10^{5}$
$C=2.17 \times 10^{-15}$
$D=2.13 \times 10^{-17}$

Tellurium 131:
$A=2.10 \times 10^{-4}$
$B=\stackrel{(\# 49)}{5.74} \times 10^{7}$
$C=1.26 \times 10^{-17}$
$D=1.24 \times 10^{-19}$

Tellurium 132:
$A=5.32 \times 10^{-3}$
$B=3.05 \times 10^{5}$
$C=6.01 \times 10^{-14}$
$D=5.90 \times 10^{-16}$

8-22. Barium 137M:
$A=3.56 \times 10^{-3}$
$B=\frac{(\# 54)}{5.38} \times 10^{8}$
$C=2.28 \times 10^{-17}$
$D=2.24 \times 10^{-19}$ 
REFERENCE CALCULATIONS: PWR Reactor (cont'd)

Barium 140:
$A=4.63 \times 10^{-5}$
$B=7.29 \times 10^{4}$
$C=2.19 \times 10^{-15}$
$D=2.15 \times 10^{-17}$

8-23. Lanthanum 140:
$A=3.22 \times 10^{-5}$
$B=\frac{(\# 56)}{5.57 \times 10^{5}}$
$c=1.99 \times 10^{-16}$
$D=1.95 \times 10^{-18}$

8-24. Cerium 141:
$A=1.46 \times 10^{-5}$
$B=2.81 \times 10^{4}$
$c=1.79 \times 10^{-15}$
$D=1.76 \times 10^{-17}$

Cerium 143:
$A=7.72 \times 10^{-6}$
$B=\frac{(\# 58)}{6.64} \times 10^{5}$
$c=4.01 \times 10^{-17}$
$D=3.93 \times 10^{-19}$
$A=7.18 \times 10^{-6}$
$B=\frac{(\# 59)}{3.18} \times 10^{3}$
$c=7.79 \times 10^{-15}$
$D=7.63 \times 10^{-17}$

Cerium 144:

8-25. Praseodymium 143:
$A=1.02 \times 10^{-5}$
$B=\frac{(\# 60)}{6.67 \times 10^{4}}$
$c=5.27 \times 10^{-16}$
$D=5.17 \times 10^{-18}$

Praseodymium 144:
$A=7.22 \times 10^{-6}$
$B=\frac{(\# 61)}{7.55} \times 10^{7}$
$C=3.30 \times 10^{-19}$
$D=3.23 \times 10^{-21}$

8-26. Neptunium 239:
$A=2.39 \times 10^{-4}$
$B=\stackrel{(\# 83)}{2.33} \times 10^{5}$
$C=3.54 \times 10^{-15}$
$D=3.47 \times 10^{-17}$

8-27. Tritium (H3):
$A=2.87 \times 10^{2}$
$B=\stackrel{(\# 70)}{9.73 \times 10^{3}}$
$C=1.02 \times 10^{-7}$
$D=9.97 \times 10^{-10}$ 
REFERENCE CALCULATIONS: PWR Reactor (cont'd)

8-28. Bromine 83:
$A=1.1 \times 10^{-3}$
$B=l^{(\# 84)} \times 1.57 \times 10^{7}$
$c=2.42 \times 10^{-16}$
$0=2.37 \times 10^{-18}$

Bromine 84:
$A=3.7 \times 10^{-5}$
$B=7^{(\# 85)} \times 10^{7}$
$C=1.81 \times 10^{-18}$
$D=1.78 \times 10^{-20}$

Bromine 85:
$A=2.5 \times 10^{-8}$
$B=\frac{(\# 99)}{7.37} \times 10^{8}$
$c=1.17 \times 10^{-22}$
$D=1.15 \times 10^{-24}$

8-29. Iodine 130:
$A=2.8 \times 10^{-3}$
$B=\frac{(\# 14)}{1.96} \times 10^{6}$
$C=4.93 \times 10^{-15}$
$D=4.83 \times 10^{-17}$
Iodine 131:
$A=1.1$
$B=\frac{(\# 15)}{1.24} \times 10^{5}$
$c=3.06 \times 10^{-11}$
$D=3.00 \times 10^{-13}$

io

Iodine 132:
$A=1.2 \times 10^{-1}$
$B=l^{(\# 16)}\left(.03 \times 10^{7}\right.$
$c=4.02 \times 10^{-14}$
$D=3.94 \times 10^{-16}$

Iodine 133:
$A=7.9 \times 10^{-1}$
$B=\left(1.13 \times 10^{6}\right.$
$c=2.41 \times 10^{-12}$
$D=2.36 \times 10^{-14}$

Iodine 134:
$A=2.0 \times 10^{-3}$
$B=\begin{aligned} & (\# 94) \\ & B=70 \times 10^{7}\end{aligned}$
$c=2.55 \times 10^{-16}$
$D=2.50 \times 10^{-18}$
Iodine 135:
$A=1.4 \times 10^{-1}$
$B=3.47 \times 10^{6}$
$c=1.39 \times 10^{-13}$
$D=1.36 \times 10^{-15}$ 
REFERENCE CALCULATIONS: PIJR Reactor (cont'd)

8-30. Rubidium 86:
$A=3.9 \times 10^{-4}$
$B=\frac{(\# 19)}{8.14} \times 10^{4}$
$C=1.65 \times 10^{-14}$
$D=1.62 \times 10-16$

Rubidium 88:
$A=4.5 E-4$
$B=1.20 \times 10^{8}$
$C=1.29 \times 10^{-17}$
$D=1.27 \times 10^{-19}$

8-31. Cesium 134:
$A=1.3 \times 10^{-1}$
$B=\frac{(\# 51)}{1.30 \times 10^{3}}$
$C=3.45 \times 10^{-10}$
$D=3.38 \times 10^{-12}$

Cesium 136:
$A=6.2 \times 10^{-2}$
$B=7.39 \times 10^{4}$
$C=2.89 \times 10^{-12}$
$D=2.84 \times 10^{-14}$

Cesium 137:
$A=1.0 \times 10^{-1}$
$B={ }^{(\# 53)} \times 10^{1}$
$c=3.99 \times 10^{-9}$
$D=3.91 \times 10^{-11}$

8-32. Chromium 51:
$A=9.0 \times 10^{-3}$
$B=\stackrel{(\# 64)}{9.21} \times 10^{4}$
$c=3.37 \times 10^{-13}$
$D=3.30 \times 10^{-15}$

8-33. Manganese 54:
$A=2.1 \times 10^{-3}$
$B=\frac{(\# 65)}{7.98 \times 10^{3}}$
$C=9.07 \times 10^{-13}$
$D=8.90 \times 10^{-15}$

8-34. Iron 55:
$A=7.6 \times 10^{-3}$
$B=2.50 \times 10^{3}$
$c=1.05 \times 10^{-11}$
$D=1.03 \times 10^{-13}$

Iron 59:
$A=4.6 \times 10^{-3}$
$B={ }^{(\# 67)} \times 10^{4}$
$c=3.23 \times 10^{-13}$
$D=3.17 \times 10^{-15}$ 


\section{REFERENCE CALCULATIONS: PWR Reactor (cont'd)}

8-35. Cobalt 58:
$A=7.6 \times 10^{-2}$
$B=3^{(\# 68)} \times 16^{4}$
$C=8.29 \times 10^{-12}$
$D=8.13 \times 10^{-14}$

Cobalt 60:
$A=1.5 \times 10^{-2}$
$\left.B=l^{(\# 69)}\right) \times 10^{3}$
$C=4.58 \times 10^{-11}$
$0=4.49 \times 10^{-13}$

8-36. Strontium 89:
$A=1.6 \times 10^{-3}$
$B=2.82 \times 10^{4}$
$C=1.96 \times 10^{-13}$
$D=1.92 \times 10^{-15}$

Strontium 90:
$A=4.7 \times 10^{-5}$
$B=l(\# 22) \times 10^{2}$
$c=1.14 \times 10^{-12}$
$D=1.12 \times 10^{-14}$

Strontium 91:
$A=7.2 \times 10^{-4}$
$B=\frac{(\# 23)}{3.56 \times 10^{6}}$
$c=6.97 \times 10^{-16}$
$D=6.84 \times 10^{-18}$

8-37. Yetrium 90:
$A=7.4 \times 10^{-5}$
$B=\frac{(\# 24)}{5.44 \times 10^{5}}$
$c=4.69 \times 10^{-16}$
$D=4.60 \times 10^{-18}$

Yttrium 91M:
$A=4.6 \times 10^{-4}$
$B=\frac{(\# 25)}{4.13} \times 10^{7}$
$c=3.84 \times 10^{-17}$
$D=3.77 \times 10^{-19}$

Yttrium 91:
$A=9.7 \times 10^{-3}$
$B=2.44 \times 10^{4}$
$c=1.37 \times 10^{-12}$
$D=1.34 \times 10^{-14}$

Yttrium 93:
$A=1.6 \times 10^{-4}$
$B=\frac{(\# 27)}{3.31} \times 10^{6}$
$c=1.67 \times 10^{-16}$
$D=1.63 \times 10^{-19}$ 
REFERENCE CALCULATIONS: PWR Reactor (cont'd)

8-38. Zirconium 95:
$A=1.1 \times 10^{-3}$
$B=\frac{(\# 28)}{2.12 \times 10^{4}}$
$c=1.79 \times 10^{-13}$
$D=1.75 \times 10^{-15}$

8-39. Niobrium 95:
$A=1.4 \times 10^{-3}$
$B=3.91 \times 10^{4}$
$c=1.23 \times 10^{-13}$
$D=1.21 \times 10^{-15}$

8-40. Molybdenum 99:

$$
A=1.6
$$

$$
B=4^{(\# 33)} \times 15^{5}
$$$$
c=1.16 \times 10^{-11}
$$$$
D=1.14 \times 10^{-13}
$$

8-41. Ruthenium 103:
$A=2.9 \times 10^{-4}$
$B=3.20 \times 10^{4}$
$C=3.13 \times 10^{-14}$
$D=3.06 \times 10^{-16}$
Ruthenium 106:
$A=4.7 \times 10^{-5}$
$B={ }^{(\# 36)} .36 \times 10^{3}$
$c=4.82 \times 10^{-14}$
$D=4.73 \times 10^{-16}$

8-42. Rhodium 103M:
$A=2.1 \times 10^{-4}$
$B=\frac{(\# 37)}{3.20} \times 10^{7}$
$C=2.26 \times 10^{-17}$
$D=2.22 \times 10^{-19}$
$A=1.3 \times 10^{-3}$
$B=\frac{(\# 39)}{3.55 \times 10^{9}}$
$C=1.26 \times 10^{-18}$
$D=1.24 \times 10^{-20}$

Rhodium 106:

8-43. Silver 110M:
$A=2.5 \times 10^{-4}$
$B=4.69 \times 10^{3}$
$C=1.84 \times 10^{-13}$
$D=1.80 \times 10^{-15}$

8-44. Tellurium 125M:
$A=1.4 \times 10^{-4}$
$B=\frac{(\# 43)}{1.80 \times 10^{4}}$
$C=2.68 \times 10^{-14}$
$D=2.63 \times 10^{-16}$ 
REFERENCE CALCULATIONS: PUR Reactor (cont'd)

Tellurium 127M:

$$
A=1.4 \times 10^{-3} \quad B=9.44 \times 10^{3} \quad C=5.11 \times 10^{-13} \quad \text { (\#44) } \quad 0=5.01 \times 10^{-15}
$$

Te11urium 127:

$$
A=2.0 \times 10^{-3} \quad B=2.63 \times 10^{6} \quad C=2.62 \times 10^{-15} \quad 0=2.57 \times 10^{-17}
$$

Tellurium 129M:

$$
A=6.4 \times 10^{-3} \quad B=2.98 \times 10^{4} \quad C=7.41 \times 10^{-13} \quad D=7.26 \times 10^{-15}
$$

Tellurium 129:

$$
A=4.2 \times 10^{-3} \quad B=2.11 \times 10^{7} \quad C=6.86 \times 10^{-16} \quad D=6.73 \times 10^{-18}
$$

Tellurium 131M:

$$
A=6.4 \times 10^{-3} \quad B=7.98 \times 10^{5} \quad C=2.77 \times 10^{-14} \quad 0=2.71 \times 10^{-16}
$$

Tellurium 131:

$$
A=1.2 \times 10^{-3} \quad B=5.74 \times 10^{7} \quad C=7.21 \times 10^{-17} \quad 0=7.07 \times 10^{-19}
$$

Tellurium 132:
$A=9.9 \times 10^{-2}$
$B=3.05 \times 10^{5}$
$c=1.12 \times 10^{-12}$
$D=1.10 \times 10^{-14}$

8-45. Barium 137M:
$A=1.1 \times 10^{-5}$
$B=\frac{(\# 54)}{5.38} \times 10^{8}$
$c=7.05 \times 10^{-20}$
$D=6.91 \times 10^{-22}$ 


\section{REFERENCE CALCULATIONS: PWR Reactor (cont'd)}

Barium 140:
$A=1.0 \times 10^{-3}$
$B=7.29 \times 10^{4}$
$C=4.73 \times 10^{-14}$
$D=4.64 \times 10^{-16}$

8-46. Lanthanum 140:
$A=8.5 \times 10^{-4}$
$B=\frac{(\# 56)}{5.57 \times 10^{5}}$
$C=5.26 \times 10^{-15}$
$D=5.16 \times 10^{-17}$

8-47. Cerium 141:
$A=3.2 \times 10^{-4}$
$B=2.81 \times 10^{4}$
$C=3.93 \times 10^{-14}$
$D=3.85 \times 10^{-16}$

Cerium 143:
$A=1.1 \times 10^{-4}$
$B=6.64 \times 10^{5}$
$c=5.71 \times 10^{-16}$
$D=5.60 \times 10^{-18}$

Cerium 144:
$A=3.0 \times 10^{-3}$
$B=\frac{(\# 59)}{3.18 \times 10^{3}}$
$c=3.25 \times 10^{-12}$
$D=3.19 \times 10^{-14}$

8-48. Praseodymium 143:
$A=2.4 \times 10^{-4}$
$B=\frac{(\# 60)}{6.67 \times 10^{4}}$
$c=1.24 \times 10^{-14}$
$D=1.22 \times 10^{-16}$

Praseodymium 144:
$A=1.6 \times 10^{-4}$
$B=\frac{(\# 61)}{7.55 \times 10^{7}}$
$C=7.31 \times 10^{-18}$
$D=7.16 \times 10^{-20}$

8-49. Neptunium 239:
$A=4.0 \times 10^{-3}$
$B=2.33 \times 10^{5}$
$C=5.92 \times 10^{-14}$
$D=5.80 \times 10^{-16}$

8-50. Tritium (H3):
$A=1010$
$B=9.73 \times 10^{3}$
$c=3.58 \times 10^{-7}$
$0=3.51 \times 10^{-9}$ 


\section{REFERENCE CALCULATIONS: PWR Reactor (cont'd)}

8-51. Summation of $D$ values in ref. calc. $8-3=\frac{5.88 \times 10^{-8}}{\mathrm{kgU} U(\text { reactor input })} \frac{\mathrm{kg} \text { kypton }}{\text { input }}$

8-52. Summation of $D$ values in ref. calc. $8-4=\frac{7.83 \times 10^{-10}}{\mathrm{~kg} \text { xenon }}$

8-53. Summation of 0 values in ref. calc. $8-5=\frac{1.86 \times 10^{-17}}{\mathrm{~kg} U(\mathrm{rgac} \text { rubidium }}$

8-54. Summation of $D$ values in ref. calc. $8-6=\frac{6.96 \times 10^{-13}}{\mathrm{~kg} U(\text { reactor }} \frac{\mathrm{kg} \text { cesium }}{\text { input })}$

8-55. Summation of $D$ values in ref. calc. $8-7=\frac{6.16 \times 10^{-19}}{\mathrm{~kg} U(\text { reactor }} \frac{\mathrm{kg} \text { bromine }}{\text { input })}$

8-56. Summation of 0 values in ref. calc. $8-8=\frac{5.39 \times 10^{-15}}{\mathrm{~kg} \text { iodine }}$ $\mathrm{kg} \mathrm{U}($ reactor input)

8-57. Summation of 0 values in ref. calc. $8-11=4.65 \times 10^{-15} \mathrm{~kg}$ iron $\mathrm{kg} \mathrm{U}($ reactor input)

8-58. Summation of $D$ values in ref. calc. $8-12=\frac{2.25 \times 10^{-14}}{\mathrm{~kg} U(\text { reactor }} \frac{\mathrm{kg} \text { cobalt }}{\text { input })}$

8-59. Summation of $D$ values in ref. calc. $8-13=\frac{1.34 \times 10^{-15}}{\mathrm{~kg} U(\text { reactor }} \frac{\mathrm{kg} \text { strontium }}{\text { input })}$

8-60. Summation of $D$ values in ref. calc. $8-14=\frac{5.80 \times 10^{-16}}{\mathrm{~kg} U(\text { reactor }} \frac{\mathrm{kg} \text { yttrium }}{\text { input })}$

8-61. Summation of $D$ values in ref. calc. $8-19=\frac{2.95 \times 10^{-17}}{\mathrm{~kg} \text { ruthenium }}$

8-62. Summation of $D$ values in ref. calc. $8-20=\frac{1.18 \times 10^{-20}}{\mathrm{~kg}} \frac{\mathrm{kg} \text { rhodium }}{\text { input })}$

8-63. Summation of $D$ values in ref. calc. $8-21=\frac{1.13 \times 10^{-15}}{\mathrm{~kg} U(\text { reactor }} \frac{\mathrm{kg} \text { tellurium }}{\text { input })}$

8-64. Summation of $D$ values in ref. calc. $8-22=\frac{2.17 \times 10^{-17}}{\mathrm{~kg} U(\text { reactor }} \frac{\mathrm{kg} \text { barium }}{\text { input })}$

8-65. Summation of $D$ values in ref. calc. $8-24=\frac{9.43 \times 10^{-17}}{\mathrm{~kg} U(\text { reactor }} \frac{\mathrm{kg} \text { cerium }}{\text { input })}$ 


\section{REFERENCE CALCULATIONS: PWR Reactor (cont'd)}

8-66. Sumination of $\mathrm{D}$ values in ref. calc. $8-25=\frac{5.17 \times 10^{-18}}{\mathrm{~kg} U(\text { reactor }} \frac{\mathrm{kg} \text { praseodymium }}{\text { input })}$

8-67. Summation of $D$ values in ref. calc. $8-28=\frac{2.39 \times 10^{-18}}{\mathrm{~kg} U(\text { reactor }}-\frac{\mathrm{kg} \text { input })}{\text { inpe }}$

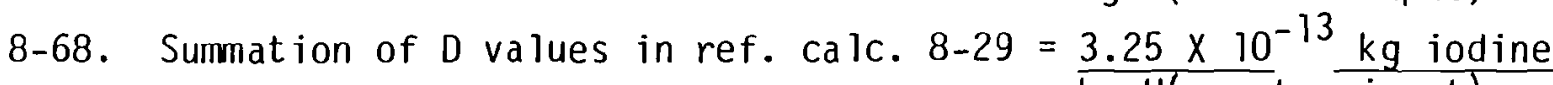

8-69. Summation of $D$ values in ref. calc. $8-30=\frac{1.62 \times 10^{-16}}{\mathrm{~kg} U(\text { reactor }} \frac{\mathrm{kg} \text { rubidium }}{\text { input })}$

8-70. Surmation of $D$ values in ref. calc. $8-31=\frac{4.25 \times 10^{-11}}{\mathrm{~kg} U(\text { reactor }} \frac{\mathrm{kg} \text { cesium }}{\text { input })}$

8-71. Summation of $D$ values in ref. calc. $8-34=\frac{1.06 \times 10^{-13}}{\mathrm{~kg} U(\text { reactor input })}$

8-72. Summation of $D$ values in ref. calc. $8-35=\frac{5.30 \times 10^{-13}}{\mathrm{~kg} U(\text { reactor }} \frac{\mathrm{kg} \text { cobalt }}{\text { input })}$

8-73. Summation of $D$ values in ref. calc. $8-36=\frac{1.31 \times 10^{-14}}{\mathrm{~kg} U(\text { reactor }} \frac{\mathrm{kg} \text { strontium }}{\text { input })}$

8-74. Summation of $D$ values in ref. calc. $8-37=\frac{1.34 \times 10^{-14}}{\mathrm{~kg} \text { yttrium }}$

8-75. Summation of $D$ values in ref. calc. $8-41=\frac{7.79 \times 10^{-16}}{\mathrm{~kg} \text { ruthenium }}$

8-76. Summation of $D$ values in ref. calc. $8-42=\frac{2.34 \times 10^{-19}}{\mathrm{~kg} U(\text { reactor }} \frac{\mathrm{kg} \text { rhodium }}{\text { input })}$

8-77. Summation of 0 values in ref. calc. $8-44=\frac{2.38 \times 10^{-14}}{\mathrm{~kg} \text { U(reactor }} \frac{\mathrm{kg} \text { tellurium }}{\text { input })}$

8-78. Summation of $D$ values in ref. calc. $8-45=\frac{4.64 \times 10^{-16}}{\mathrm{~kg} U(\text { reactor }} \frac{\mathrm{kg} \text { barium }}{\text { input })}$

8-79. Summation of $D$ values in ref. calc. $8-47=\frac{3.23 \times 10^{-14}}{\mathrm{~kg} U(\text { reactor cerium }}$

8-80. Summation of $\mathrm{D}$ values in ref. calc. $8-48=1.22 \times 10^{-16} \mathrm{~kg}$ praseodymium $\mathrm{kg} \mathrm{U}($ reactor input) 
REFERENCE CALCULATIONS: PWR Reactor (cont'd)

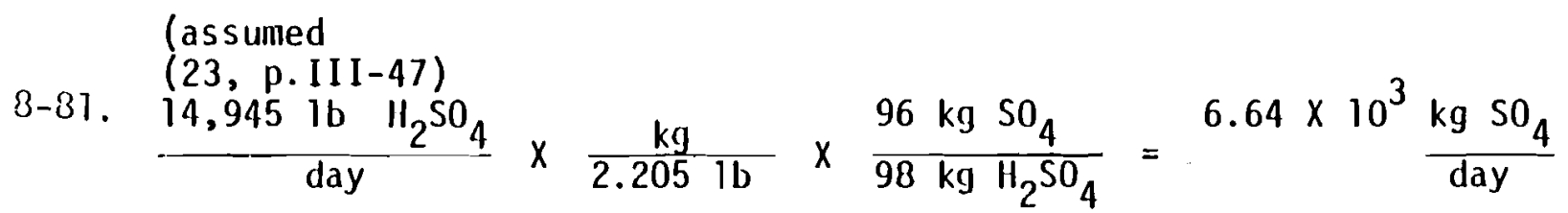

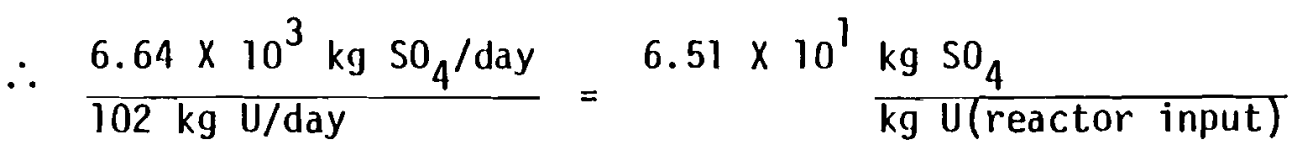

(assumed

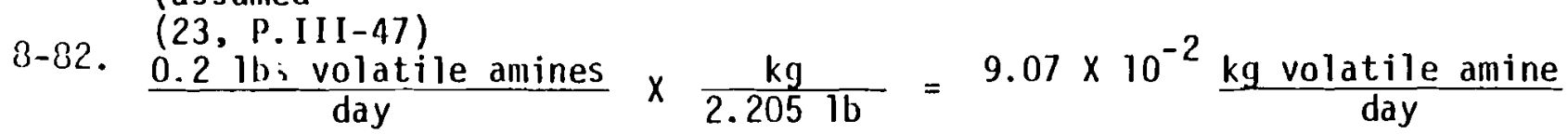

$\stackrel{P}{0}$

$\therefore \quad \frac{9.07 \times 10^{-2}}{102 \mathrm{~kg} \text { U/day volatile amine/day }}=8.89 \times 10^{-4} \frac{\mathrm{kg} \text { volatile amine }}{\mathrm{kg} \text { U(reactor input })}$

(assumed

(23, p.III-47)

3-33. $\frac{8.05 \mathrm{lb} \mathrm{H} \mathrm{H}_{3} \mathrm{BO}_{3}}{\text { day }} \times \frac{\mathrm{kg}}{2.205 \mathrm{lb}} \times \frac{11 \mathrm{~kg} \mathrm{~B}}{62 \mathrm{~kg} \mathrm{H}_{3} \mathrm{BO}_{3}}=6.48 \times 10^{-1} \frac{\mathrm{kg} \mathrm{B}}{\text { day }}$

$\therefore \frac{6.48 \times 10^{-1} \mathrm{~kg} \mathrm{~B} / \text { day }}{102 \mathrm{~kg} \mathrm{U} / \text { day }}=6.35 \times 10^{-3} \frac{\mathrm{kg} \mathrm{B}}{\mathrm{kg} \mathrm{U}(\text { reactor input })}$ 


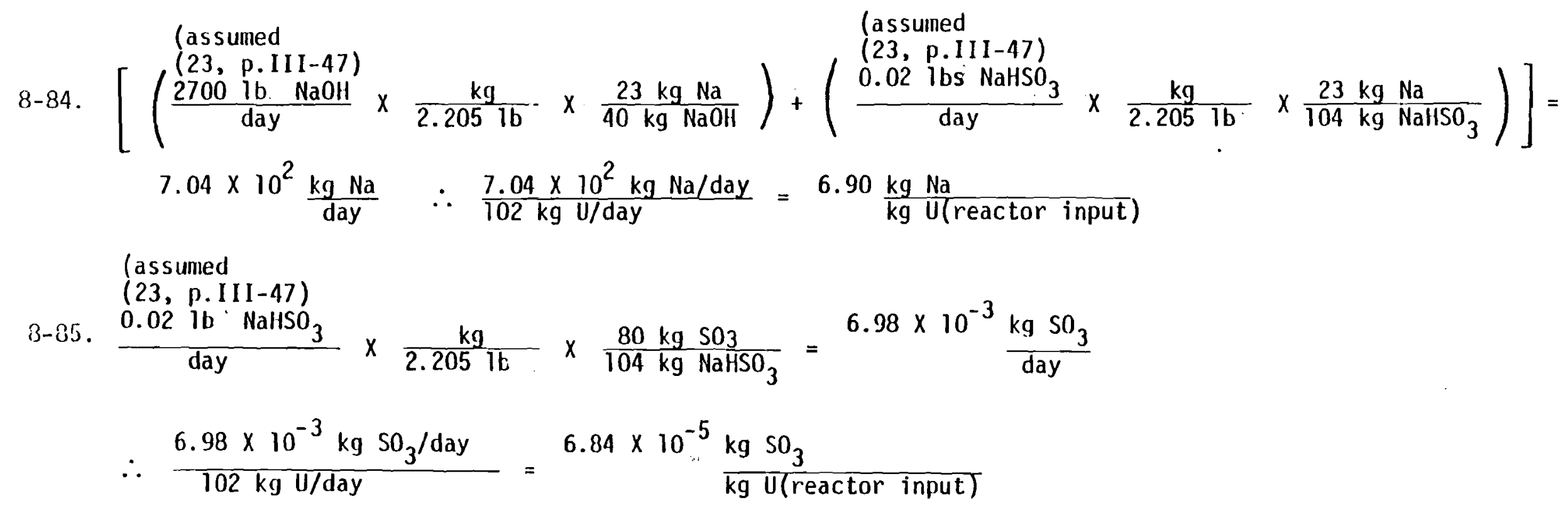


REFERENCE CALCULATIONS: PHR Reactor (cont'd)

\begin{tabular}{|c|c|c|c|c|}
\hline & Isotope & D & GA & $\mathrm{RF}$ \\
\hline 1) & Argon-41 & $2.02 \mathrm{E}-14$ & 4. $38 \mathrm{E}-16$ & 1.0 \\
\hline 2) & Krypton-83M & 1. $38 \mathrm{E}-15$ & $3.46 \mathrm{E}-8$ & $4.8 E-7$ \\
\hline 3) & Krypton-85M & $1.68 \mathrm{E}-14$ & $2.58 \mathrm{E}-7$ & $7.7 \mathrm{E}-7$ \\
\hline 4) & Krypton-85 & $5.88 \mathrm{E}-8$ & 3. $38 \mathrm{E}-4$ & $2.0 \mathrm{E}-3$ \\
\hline 5) & Krypton-87 & $2.73 E-15$ & $1.43 E-7$ & $2.2 \mathrm{E}-7$ \\
\hline 6) & Krypton-88 & $2.08 \mathrm{E}-14$ & $4.62 E-7$ & $5.1 \mathrm{E}-7$ \\
\hline 7) & Krypton-89 & $9.68 \mathrm{E}-18$ & 1. $10 \mathrm{E}-8$ & $9.9 \mathrm{E}-9$ \\
\hline 8) & Xenon-131M & $1.16 \mathrm{E}-10$ & $6.15 E-7$ & $1.4 \mathrm{E}-3$ \\
\hline 9) & Xenon-133M & $1.37 E-12$ & $6.57 \mathrm{E}-7$ & $1.6 \mathrm{E}-5$ \\
\hline 10) & Xenon-133 & $6.65 E-10$ & $6.32 E-5$ & $7.9 \mathrm{E}-5$ \\
\hline 11) & Xenon-135M & $1.79 \mathrm{E}-16$ & $3.48 \mathrm{E}-8$ & $3.8 \mathrm{E}-8$ \\
\hline 12) & Xenon-135 & $2.09 E-13$ & $1.08 \mathrm{E}-6$ & $1.4 \mathrm{E}-6$ \\
\hline 13) & Xenon-137 & $3.23 E-17$ & $3.17 \mathrm{E}-8$ & $7.4 E-9$ \\
\hline 14) & Xenon-138 & $6.79 \mathrm{E}-16$ & 1. $33 \mathrm{E}-7$ & $3.7 E-8$ \\
\hline 15) & Rubidium-86 & $8.14 E-18$ & $6.65 E-8$ & $1.4 \mathrm{E}-9$ \\
\hline 16) & Rubidium-88 & $1.05 \mathrm{E}-17$ & $4.89 E-8$ & $2.4 \mathrm{E}-9$ \\
\hline 17) & Cesium-134 & $4.21 E-14$ & $1.41 \mathrm{E}-3$ & $2.2 \mathrm{E}-10$ \\
\hline 18) & Cesium-136 & 1. $35 \mathrm{E}-15$ & $6.00 E-6$ & $1.7 \mathrm{E}-9$ \\
\hline 19) & Cesium-137 & $6.53 \mathrm{E}-13$ & $9.06 \mathrm{E}-3$ & $5.9 \mathrm{E}-10$ \\
\hline 20) & Bromine-83 & $5.51 \mathrm{E}-19$ & $4.49 \mathrm{E}-8$ & $1.5 \mathrm{E}-10$ \\
\hline
\end{tabular}


REFERENCE CALCULATIONS: PWR Reactor (cont'd)

\begin{tabular}{ll}
\multicolumn{2}{c}{ Isotope } \\
\hline 21) & Bromine- 84 \\
22) & Bromine-85 \\
23) & Iodine-130 \\
24) & Iodirie-131 \\
25) & Iodine-132 \\
26) & Iodine-133 \\
27) & Iodine-134 \\
28) & Iodine-135 \\
29) & Chromium-51 \\
30) & Manganese-54 \\
31) & Iron-55 \\
32) & Iron-59 \\
33) & Cobalt-58 \\
34) & Cobalt-60 \\
35) & Strontium-89 \\
36) & Strontium-90 \\
37) & Strontium-91 \\
38) & Yttrium-90 \\
39) & Yttrium-91M \\
40) & Yttrium-91 \\
41) & Yttrium-93
\end{tabular}

\begin{tabular}{c}
$D$ \\
\hline $6.39 E-20$ \\
$7.25 E-22$ \\
$2.00 E-18$ \\
$4.69 E-15$ \\
$1.83 E-17$ \\
$5.80 E-16$ \\
$3.06 E-18$ \\
$1.00 E-16$ \\
$1.53 E-16$ \\
$1.80 E-15$ \\
$4.53 E-15$ \\
$1.22 E-16$ \\
$2.04 E-15$ \\
$2.05 E-14$ \\
$3.85 E-17$ \\
$1.30 E-15$ \\
$1.17 E-18$ \\
$2.08 E-19$ \\
$5.93 E-20$ \\
$5.79 E-16$ \\
$2.47 E-19$ \\
\end{tabular}

\begin{tabular}{l} 
GA \\
\hline $2.51 E-8$ \\
$2.61 E-9$ \\
$1.27 E-7$ \\
$5.19 E-5$ \\
$8.79 E-7$ \\
$1.05 E-5$ \\
$5.00 E-17$ \\
$2.96 E-6$ \\
$5.39 E-8$ \\
$1.90 E-8$ \\
$3.04 E-17$ \\
$1.12 E-9$ \\
$3.12 E-8$ \\
$9.29 E-9$ \\
$2.82 E-4$ \\
$6.03 E-3$ \\
$2.73 E-6$ \\
$1.63 E-6$ \\
$1.39 E-7$ \\
$4.19 E-4$ \\
$3.59 E-6$
\end{tabular}

\begin{tabular}{l}
$\mathrm{RF}$ \\
\hline $3.0 \mathrm{E}-11$ \\
$3.3 \mathrm{E}-12$ \\
$1.2 \mathrm{E}-10$ \\
$6.9 \mathrm{E}-10$ \\
$1.6 \mathrm{E}-10$ \\
$4.2 \mathrm{E}-10$ \\
$4.6 \mathrm{E}-11$ \\
$2.5 \mathrm{E}-10$ \\
$5.6 \mathrm{E}-8$ \\
$1.8 \mathrm{E}-6$ \\
$2.7 \mathrm{E}-7$ \\
$1.8 \mathrm{E}-6$ \\
$1.1 \mathrm{E}-6$ \\
$3.7 \mathrm{E}-5$ \\
$1.5 \mathrm{E}-12$ \\
$2.4 \mathrm{E}-12$ \\
$4.7 \mathrm{E}-12$ \\
$1.4 \mathrm{E}-12$ \\
$4.7 \mathrm{E}-12$ \\
$1.5 \mathrm{E}-11$ \\
$7.4 \mathrm{E}-13$
\end{tabular}


REFERENCE CALCULATIONS: PWR Reactor (cont'd)

\begin{tabular}{|c|c|c|c|c|}
\hline & Isotope & $D$ & GA & $\mathrm{RF}$ \\
\hline 42) & Zirconium-95 & $2.17 E-17$ & $6.74 E-4$ & $3.4 E-13$ \\
\hline 43) & Niobium-95 & $9.94 E-18$ & $3.69 E-4$ & $2.8 \mathrm{E}-13$ \\
\hline 44) & Mo 1 ybdenum-99 & $6.02 E-15$ & $3.19 \mathrm{E}-5$ & $1.9 \mathrm{E}-9$ \\
\hline 45) & Technetium-99M & $4.78 E-16$ & $2.49 \mathrm{E}-6$ & $1.9 E-9$ \\
\hline 46) & Ruthen ium- 103 & $9.69 \mathrm{E}-18$ & $3.65 \mathrm{E}-4$ & $2.6 \mathrm{E}-13$ \\
\hline 47) & Ruthen ium- 106 & $1.93 E-17$ & $1.55 E-3$ & $1.2 E-13$ \\
\hline 48) & Rhodium-103M & $9.63 \mathrm{E}-21$ & $3.65 E-7$ & $2.6 \mathrm{E}-13$ \\
\hline 49) & Rhodium-106 & $2.15 E-21$ & $1.95 E-9$ & $1.0 \mathrm{E}-11$ \\
\hline 50) & Tellurium-125M & $1.06 \mathrm{E}-17$ & $1.41 \mathrm{E}-6$ & $6.0 E-11$ \\
\hline 51) & Tellurium-127M & $1.99 \mathrm{E}-16$ & $1.26 \mathrm{E}-5$ & 1. $2 E-10$ \\
\hline 52) & Tellurium-127 & $2.15 E-18$ & $2.09 \mathrm{E}-7$ & $8.1 E-11$ \\
\hline 53) & Tellurium-129M & $3.06 E-16$ & $1.45 E-5$ & $1.6 \mathrm{E}-10$ \\
\hline 54) & Tellurium-129 & $5.01 E-19$ & $1.20 E-7$ & $3.2 E-11$ \\
\hline 55) & Tellurium-131M & $2.13 \mathrm{E}-17$ & $1.20 \mathrm{E}-6$ & $1.4 E-10$ \\
\hline 56) & Tellurium-131 & $1.24 \mathrm{E}-19$ & $9.82 \mathrm{E}-8$ & $9.6 \mathrm{E}-12$ \\
\hline 57) & Te11urium-132 & $5.90 \mathrm{E}-16$ & $2.88 \mathrm{E}-5$ & $1.6 \mathrm{E}-10$ \\
\hline 58) & Barium-137M & $2.24 E-19$ & $3.17 E-4$ & $5.2 E-15$ \\
\hline 59) & Barium- 140 & $2.15 \mathrm{E}-17$ & $1.39 \mathrm{E}-4$ & $1.1 \mathrm{E}-12$ \\
\hline 60$)$ & Lanthanum- 140 & $1.95 \mathrm{E}-18$ & $1.87 E-5$ & $7.4 \mathrm{E}-13$ \\
\hline 61) & Cerium-141 & $1.76 E-17$ & $3.38 \mathrm{E}-4$ & $3.7 E-13$ \\
\hline 62) & Cerium-143 & $3.93 \mathrm{E}-19$ & $1.25 E-5$ & $2.2 E-13$ \\
\hline 63) & Cerium-144 & $7.63 \mathrm{E}-17$ & $2.40 \mathrm{E}-3$ & $2.2 \mathrm{E}-13$ \\
\hline 64) & Praseodymium- 143 & $5.17 E-18$ & $1.24 E-4$ & $2.9 \mathrm{E}-13$ \\
\hline
\end{tabular}


REFERENCE CALCULATIONS: PIVR Reactor (cont'd)

\begin{tabular}{|c|c|c|c|c|}
\hline & I sotope & $\mathrm{D}$ & GA & RF \\
\hline 65) & Praseodymium-144 & $3.23 \mathrm{E}-21$ & $1.02 \mathrm{E}-7$ & $2.2 \mathrm{E}-13$ \\
\hline 66) & Neptunium-239 & $3.47 \mathrm{E}-17$ & $3.20 \mathrm{E}-4$ & $4.5 E-13$ \\
\hline 67) & $\operatorname{Tritium}(H-3)$ & $9.97 E-10$ & $1.64 \mathrm{E}-5$ & $2.0 \mathrm{E}-2$ \\
\hline 68) & Bromine-83 & $2.37 E-18$ & $4.49 E-8$ & $6.4 \mathrm{E}-10$ \\
\hline 69) & Bromine-84 & $1.78 \mathrm{E}-20$ & $2.51 E-8$ & $8.4 E-12$ \\
\hline 70) & Bromine-85 & $1.15 \mathrm{E}-24$ & $2.61 E-9$ & $5.2 E-15$ \\
\hline 71) & Iodine-130 & $4.83 E-17$ & $1.27 \mathrm{E}-7$ & $2.9 \mathrm{E}-9$ \\
\hline 72) & Iodine-131 & $3.00 E-13$ & $5.19 E-5$ & $4.4 E-8$ \\
\hline 73) & Iodine-132 & $3.94 E-16$ & $8.79 E-7$ & $3.4 \mathrm{E}-9$ \\
\hline 74) & Iodine-133 & $2.36 \mathrm{E}-14$ & $1.05 \mathrm{E}-5$ & $1.7 \mathrm{E}-8$ \\
\hline 75) & Iodine-134 & $2.50 E-18$ & $5.00 \mathrm{E}-7$ & $3.7 \mathrm{E}-11$ \\
\hline 76) & Iodine-135 & $1.36 \mathrm{E}-15$ & $2.96 \mathrm{E}-6$ & $3.4 \mathrm{E}-9$ \\
\hline 77) & Rubidium-86 & $1.62 E-16$ & $6.65 \mathrm{E}-8$ & $2.8 \mathrm{E}-8$ \\
\hline 78) & Rubidium-88 & $1.27 E-19$ & $4.89 \mathrm{E}-8$ & $3.0 \mathrm{E}-11$ \\
\hline 79) & Cesium-134 & $3.38 \mathrm{E}-12$ & $1.41 \mathrm{E}-3$ & $1.8 \mathrm{E}-8$ \\
\hline 80) & Cesium-136 & $2.84 E-14$ & $6.00 E-6$ & $3.5 \mathrm{E}-8$ \\
\hline 81) & Cesium-137 & $3.91 E-11$ & $9.06 \mathrm{E}-3$ & $3.2 \mathrm{E}-8$ \\
\hline 82) & Chromium-51 & $3.30 \mathrm{E}-15$ & $5.39 \mathrm{E}-8$ & $1.2 \mathrm{E}-6$ \\
\hline 83) & Manganese-54 & $8.90 E-15$ & $1.90 \mathrm{E}-8$ & $8.7 \mathrm{E}-6$ \\
\hline 84) & Iron-55 & $1.03 E-13$ & $3.04 \mathrm{E}-7$ & $6.2 E-6$ \\
\hline 85) & Iron-59 & $3.17 \mathrm{E}-15$ & $1.12 \mathrm{E}-9$ & $4.8 E-5$ \\
\hline 86) & Cobalt-58 & $8.13 E-14$ & $3.12 \mathrm{E}-8$ & $4.5 E-5$ \\
\hline 87) & Coba $1 \mathrm{t}-60$ & $4.49 E-13$ & $9.29 E-9$ & 8. $1 E-4$ \\
\hline 88) & Strontium- 89 & $1.92 E-15$ & $2.82 \mathrm{E}-4$ & $7.7 \mathrm{E}-11$ \\
\hline
\end{tabular}


REFERENCE CALCULATIONS: PWR Reactor (cont'd)

\begin{tabular}{|c|c|c|c|c|}
\hline \multicolumn{2}{|c|}{ Isotope } & D & $\mathrm{GA}$ & $\mathrm{RF}$ \\
\hline 89) & Strontium-90 & $1.12 \mathrm{E}-14$ & $6.03 E-3$ & $2.1 E-11$ \\
\hline 90) & Strontium-91 & $6.84 E-18$ & $2.73 E-16$ & $2.8 \mathrm{E}-11$ \\
\hline 91) & Yttrium-90 & $4.60 E-18$ & $1.63 \mathrm{E}-6$ & $3.1 \mathrm{E}-11$ \\
\hline 92) & Yttrium-91M & $3.77 \mathrm{E}-19$ & $1.39 \mathrm{E}-7$ & $3.0 E-11$ \\
\hline 93) & Yttrium-9l & $1.34 \mathrm{E}-14$ & $4.19 \mathrm{E}-4$ & $3.5 \mathrm{E}-10$ \\
\hline 94) & Yttrium-93 & $1.63 \mathrm{E}-19$ & $3.59 \mathrm{E}-6$ & $4.9 \mathrm{E}-13$ \\
\hline 95) & Zirconium-95 & $1.75 E-15$ & $6.74 \mathrm{E}-4$ & $2.7 E-11$ \\
\hline 96) & Niobium-95 & $1.21 \mathrm{E}-15$ & $3.68 \mathrm{E}-4$ & $3.5 \mathrm{E}-11$ \\
\hline 97) & Mo lybdenum-99 & $1.14 E-13$ & $3.19 \mathrm{E}-5$ & $3.6 \mathrm{E}-8$ \\
\hline 98) & Rutheni ium- 103 & $3.06 \mathrm{E}-16$ & $3.65 \mathrm{E}-4$ & $8.1 E-12$ \\
\hline 99) & Ruthen ium-106 & $4.73 E-16$ & $1.55 \mathrm{E}-3$ & $2.9 \mathrm{E}-12$ \\
\hline 100) & Rhodium-103M & $2.22 E-19$ & $3.65 \mathrm{E}-7$ & $5.9 \mathrm{E}-12$ \\
\hline 101) & Rhodium-106 & $1.24 \mathrm{E}-20$ & $1.95 \mathrm{E}-9$ & $6.0 \mathrm{E}-11$ \\
\hline 102) & Silver-110M & $1.80 E-15$ & $7.12 \mathrm{E}-6$ & $2.3 \mathrm{E}-9$ \\
\hline 103) & Tellurium-125M & $2.63 E-16$ & $1.41 \mathrm{E}-6$ & $1.5 \mathrm{E}-9$ \\
\hline 104) & Tellurium-127M & $5.01 E-15$ & $1.26 \mathrm{E}-5$ & $3.1 E-9$ \\
\hline 105) & Tellurium-127 & $2.57 E-17$ & $2.09 \mathrm{E}-7$ & $9.7 \mathrm{E}-10$ \\
\hline 106) & Tellurium-129M & $7.26 \mathrm{E}-15$ & $1.45 E-5$ & $3.9 \mathrm{E}-9$ \\
\hline 107) & Tellurium-129 & $6.73 E-18$ & $1.20 \mathrm{E}-7$ & $4.3 \mathrm{E}-10$ \\
\hline 108) & Tellurium-131M & $2.71 \mathrm{E}-16$ & $1.20 \mathrm{E}-6$ & $1.7 E-9$ \\
\hline 109) & Tellurium-131 & $7.07 E-19$ & $9.82 E-8$ & $5.5 E-11$ \\
\hline 110) & Tellurium-132 & $1.10 E-14$ & $2.88 E-5$ & $2.9 E-9$ \\
\hline
\end{tabular}


REFERENCE CALCULATIONS: PWR Reactor (cont'd)

\begin{tabular}{|c|c|c|c|c|}
\hline & I sotope & D & GA & $\mathrm{RF}$ \\
\hline 111$)$ & Barium-137M & $6.9 E-22$ & $3.17 E-4$ & $1.6 \mathrm{E}-17$ \\
\hline 112) & Barium-140 & $4.64 E-16$ & $1.39 E-4$ & $2.4 E-11$ \\
\hline 113) & Lanthanum- 140 & $5.16 \mathrm{E}-17$ & $1.87 E-5$ & $2.0 \mathrm{E}-11$ \\
\hline 114) & Cerium-141 & $3.85 E-16$ & $3.38 E-4$ & $8.1 E-12$ \\
\hline 115) & Cerium-143 & $5.60 E-18$ & $1.25 E-5$ & $3.1 E-12$ \\
\hline 116) & Cerium-144 & $3.19 E-14$ & $2.40 E-3$ & $9.2 \mathrm{E}-11$ \\
\hline 117) & Praseodymium- 143 & $1.22 E-16$ & $1.24 \mathrm{E}-4$ & $6.9 \mathrm{E}-12$ \\
\hline 118) & Praseodymium- 144 & $7.16 E-20$ & $1.02 E-7$ & $4.9 E-12$ \\
\hline 119) & Neptunium-239 & $5.80 E-16$ & $3.20 \mathrm{E}-4$ & $7.6 E-12$ \\
\hline 120) & Tritium $(\mathrm{H}-3)$ & $3.51 E-9$ & $1.64 \mathrm{E}-5$ & $7.1 E-2$ \\
\hline
\end{tabular}

\footnotetext{
8-87. $\frac{5 \text { lb orthophosphate }}{\text { day }} \times \frac{\mathrm{kg}}{2,205 \mathrm{lb}}=\frac{2.27 \mathrm{~kg} \text { orthophosphate }}{\text { day }}$

$\therefore \frac{2.27 \mathrm{~kg} \mathrm{orthophosphate} / \text { day }}{102 \mathrm{~kg} \mathrm{U} / \text { day }}=2.22 \times 10^{-2} \frac{\mathrm{kg} \text { orthophosphate }}{\mathrm{kg} \mathrm{U(reactor} \mathrm{input})}$
} 


\begin{tabular}{|c|c|c|c|c|c|c|}
\hline \multirow[b]{2}{*}{ Material Released } & \multicolumn{3}{|c|}{ Air Borne } & \multicolumn{3}{|c|}{ Liquid } \\
\hline & Factor & Reliability & $\begin{array}{l}\text { Ref. } \\
\text { Calc. }\end{array}$ & Factor & Reliability & $\begin{array}{l}\text { Ref. } \\
\text { Calc. }\end{array}$ \\
\hline Calcium (Ca) & & & & $2 \times 10^{1}$ & 2 & $9-47$ \\
\hline Magnesium (Mg) & & & & 4 & 2 & $9-48$ \\
\hline Sodium ( $\mathrm{Na}$ ) & & & & 2 & 2 & $9-49$ \\
\hline Bicarbonate $\left(\mathrm{HCO}_{3}\right)$ & & & & $2 \times 10^{1}$ & 2 & $9-50$ \\
\hline Sulfate $\left(\mathrm{SO}_{4}\right)$ & & & & $4 \times 10^{1}$ & 2 & $9-51$ \\
\hline Chloride (Cl) & & & & $8 \times 10^{-1}$ & 2 & $9-52$ \\
\hline Nitrate $\left(\mathrm{NO}_{3}\right)$ & & & & $2 \times 10^{-1}$ & 2 & $9-53$ \\
\hline Phosphate $\left(\mathrm{PO}_{4}\right)$ & & & & $1 \times 10^{-2}$ & 2 & $9-54$ \\
\hline Chlorine $\left(\mathrm{Cl}_{2}\right)$ & & & & $8 \times 10^{-3}$ & 1 & $9-55$ \\
\hline
\end{tabular}


BWR REACTOR RADIONUCLIDE RELEASE FACTOR

Material Releaser
Argon
*Argon - 41
Krypton
Krypton - $83 \mathrm{M}$
Krypton - $85 \mathrm{M}$
Krypton - 85
Krypton - 87
Krypton - 88
Krypton - 89
Xenon
Xenon - $131 \mathrm{M}$
Xenon - $133 \mathrm{M}$
Xenon - 133
Xenon - $135 \mathrm{M}$
Xenon - 135
Xenon - 137
Xenon - 138

Air Borne

$1 \times 10^{-6}$

$3 \times 10^{-5}$

$1 \times 10^{-3}$

$4 \times 10^{-5}$

$3 \times 10^{-5}$

$1 \times 10^{-7}$

$5 \times 10^{-5}$

$2 \times 10^{-6}$

$1 \times 10^{-4}$

$1 \times 10^{-4}$

$2 \times 10^{-4}$

$4 \times 10^{-8}$

$6 \times 10^{-5}$ $\frac{\mathrm{kg} \text { Effluent }}{\mathrm{kg} \text { Contained }}$ OR $\frac{\mathrm{Ci} \text { Effluent }}{\mathrm{Ci} \text { Contained }}$ $F-5-16-77$ Liquid

Ref.

Factor

9-56-1

1

2

2

9-56-2

9-56-3

9-56-4

9-56-5

9-56-6

9-56-7

$9-56-8$

9-56-9

9-56-10

$9-56-11$

9-56-12

9-56-13

9-56-14 


\begin{tabular}{|c|c|c|c|c|c|c|}
\hline & & Air Borne & $\overline{\mathrm{kg}}$ Conta & $\overline{C i}$ Conta & Liquid & \\
\hline Material Released & Factor & Reliability & $\begin{array}{l}\text { Ref. } \\
\text { Calc. }\end{array}$ & Factor & Reliability & $\begin{array}{l}\text { Ref. } \\
\text { Calc. }\end{array}$ \\
\hline Iodine & & & & & & \\
\hline Iodine - 131 & $4 \times 10^{8}$ & 2 & $9-56-15$ & $6 \times 10^{-11}$ & 2 & $9-56-75$ \\
\hline Iodine -132 & & & & $2 \times 10^{-11}$ & 2 & $9-56-76$ \\
\hline Iodine -133 & $7 \times 10^{-8}$ & 2 & $9-56-16$ & $8 \times 10^{-11}$ & 2 & $9-56-77$ \\
\hline Iodine - 134 & & & & $6 \times 10^{-12}$ & 2 & $9-56-78$ \\
\hline Iodine - 135 & & & & $4 \times 10^{-11}$ & 2 & $9-56-79$ \\
\hline Chromium & & & & & & \\
\hline Chromium - 51 & $3 \times 10^{-6}$ & 2 & $9-56-17$ & $2 \times 10^{-7}$ & 2 & $9-56-36$ \\
\hline Mang anese & & & & & & \\
\hline Manganese - 54 & $3 \times 10^{-5}$ & 2 & $9-56-18$ & $7 \times 10^{-8}$ & 2 & $9-56-37$ \\
\hline Manganese - 56 & & & & $2 \times 10^{-7}$ & 2 & $9-56-38$ \\
\hline Iron & & & & & & \\
\hline Iron - 55 & & & & $2 \times 10^{-7}$ & 2 & $9-56-39$ \\
\hline Iron -59 & $2 \times 10^{-5}$ & 2 & $9-56-19$ & $8 \times 10^{-8}$ & 2 & $9-56-40$ \\
\hline Cobalt & & & & & & \\
\hline Cobalt - 58 & $1 \times 10^{-6}$ & 2 & $9-56-20$ & $3 \times 10^{-8}$ & 2 & $9-56-41$ \\
\hline Coblat - 60 & $1 \times 10^{-3}$ & 1 & $9-56-21$ & $6 \times 10^{-6}$ & 2 & $9-56-42$ \\
\hline Zinc & & & & & & \\
\hline Zinc -65 & $5 \times 10^{-3}$ & 1 & $9-56-22$ & $7 \times 10^{-5}$ & 2 & $9-56-45$ \\
\hline Zinc $-69 M$ & & & & 0 & 1 & $9-56-46$ \\
\hline Zinc -69 & & & & 0 & 1 & $9-56-47$ \\
\hline
\end{tabular}




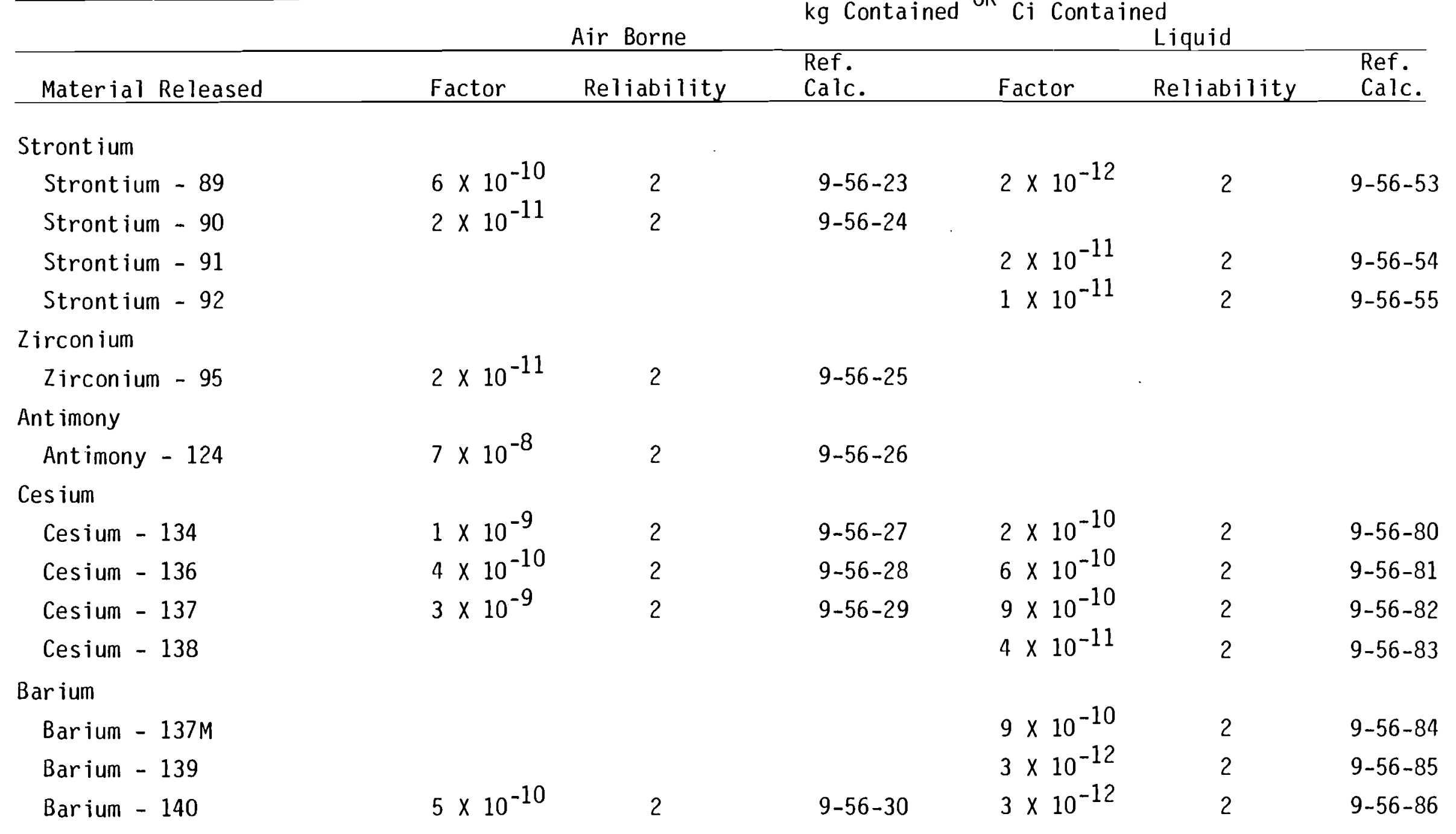


BWR REACTOR RADIONUCLIDE RELEASE FACTOR

Material Released
Cerium
Cerium - 141
Cerium - 143
Tritium
Carbon
Carbon - 14
Sodium
Sodium - 24
Phosphorus
Phosphorus - 32
Nickel
Nickel - 65
Copper
Copper - 64
Wolfram
Wolfram - 187
Neptunium
Neptunium - 239

Air Borne Factor Reliability $\frac{\mathrm{kg} \text { Effluent }}{\mathrm{kg} \text { Contained }}$ OR $\frac{\mathrm{C} i \text { Effluent }}{\mathrm{C} i \text { Contained }}$ Liquid

$F-5-16-77$

\begin{tabular}{llll}
\multicolumn{3}{c}{ Liquid } \\
Ref. & & & Ref. \\
Calc. & Factor & Reliability & Calc. \\
\hline
\end{tabular}

$\begin{array}{llllll}4 \times 10^{-11} & 2 & 9-56-31 & 2 \times 10^{-13} & 2 & 9-56-90 \\ 7 \times 10^{-3} & 2 & & 2 \times 10^{-13} & 2 & 9-56-91 \\ & 2 & 9-56-32 & 1 \times 10^{-3} & 2 & 9-56-93\end{array}$

1

1

$9-56-33$

$5 \times 10^{-5}$

2

$9-56-34$

$2 \times 10^{-6}$

2

$9-56-35$

$6 \times 10^{-8}$

2

$9-56-43$

$2 \times 10^{-6}$

2

$9-56-44$

$9 \times 10^{-9}$

2

$9-56-48$

$3 \times 10^{-12}$

2

$9-56-49$ 


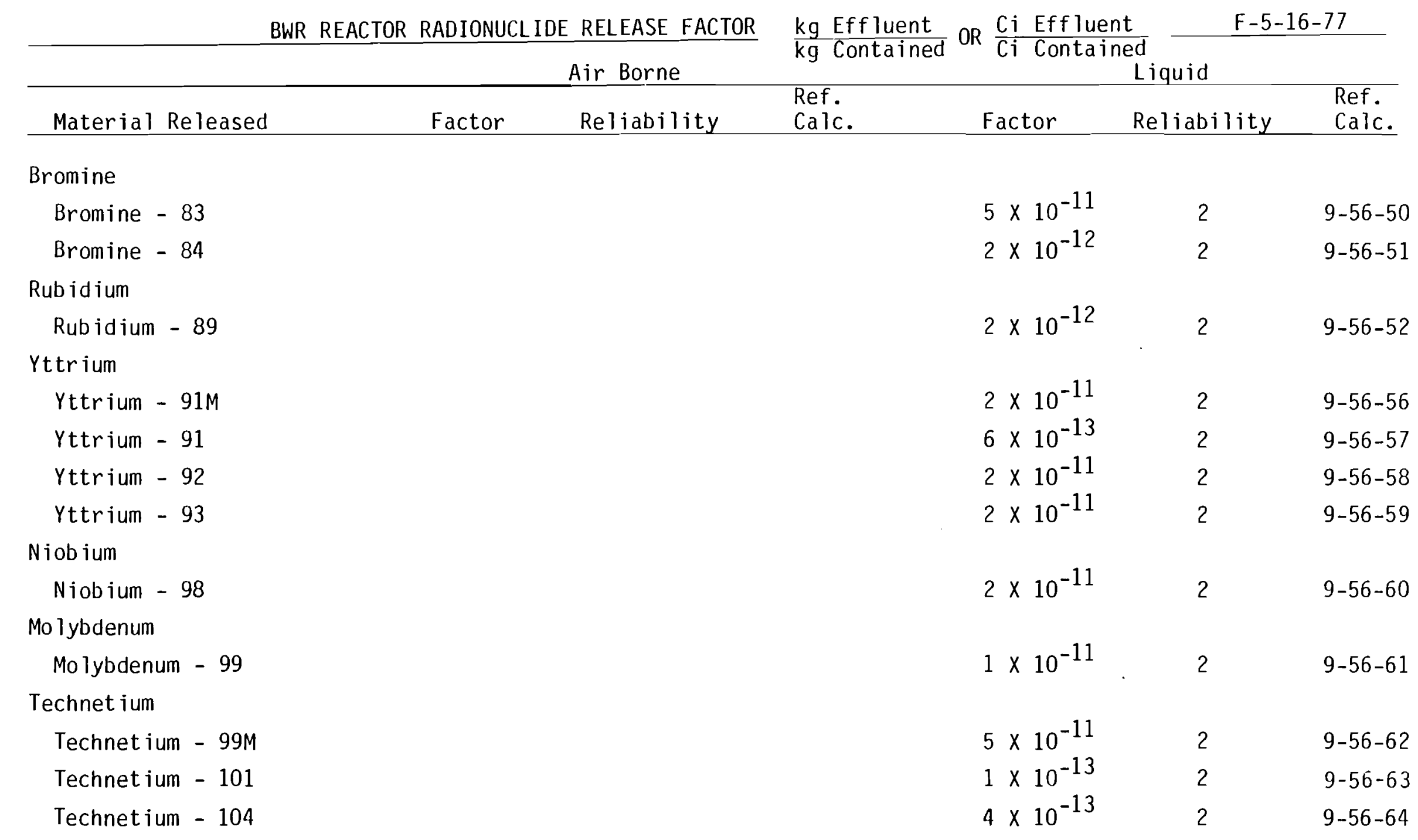




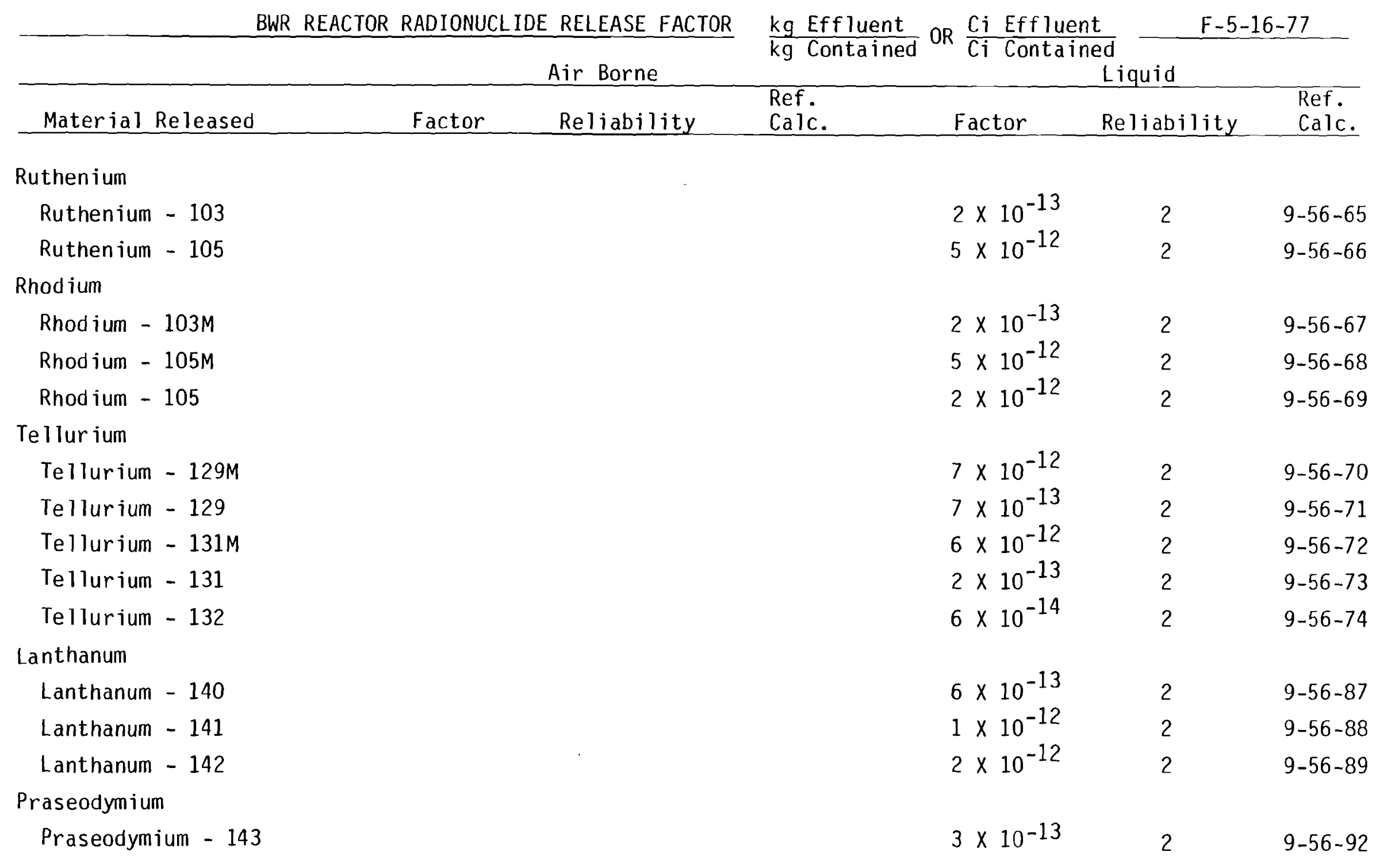




\section{REFERENCE CALCULATIONS: BWR REACTOR (292 Operating Days/yr)}

5-10-77

9-1. U input to the BWR reactor assuming $1 / 5$ of the fuel is changed out every year:

$\begin{array}{ll}(25, \mathrm{p.} 11-45) & \text { (see above) } \\ 139,344 \mathrm{~kg} \mathrm{U0} & \times \frac{238 \mathrm{U}}{270 \mathrm{UO}_{2}} \times \frac{1 / 5}{1} \times \frac{\text { year }}{292 \text { operating days }}=84 \frac{\mathrm{kg} \mathrm{U}}{\text { day }}\end{array}$

Reference calculations 9-2 through 9-46 use the following format for calculation of numbers:

$\underline{\text { A Ci radionuclide }} \times \frac{\mathrm{yr}}{292 \mathrm{op} . \text { days }} \times \frac{\text { g radionuclide }}{\underline{B} \mathrm{Ci} \text { radionuclide }} \times \frac{\mathrm{kg}}{10^{3} \mathrm{~g}}=\frac{\mathrm{C} \mathrm{kg} \text { radionuclide }}{\text { operating days }}$

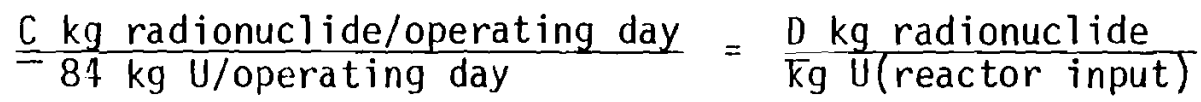

Note: Primary coolant activity based on an 0.80 plant load factor and a noble gas release rate of $10^{5} \mathrm{Hi} / \mathrm{sec}$ after a 30 -minute decay.

A11 A values in reference calculations 9-3 through 9-18 are from reference 33, tables 11.3-7 and 11.3-9; all A values in reference calculations 9-19 through 9-46 are from reference 33, tables. 11.2-7 and $11.2-9$.

A11 B values in reference calculations 9-2 through 9-46 are from reference 24.

9-2. Argon 41: (assumed)
$A=2.5 \times 10^{1}$
$B=4.18 \times 10^{7}$
$C=2.05 \times 10^{-12}$
$D=2.44 \times 10^{-14}$

9.3. Krypton 83M:

$A=1.0$

$B=2.03 \times 10^{7}$

$c=1.69 \times 10^{13}$

$D=2.01 \times 10^{-14}$

Krypton 85M:
$A=7.6 \times 10^{1}$
$B=8.40 \times 10^{6}$
$c=3.10 \times 10^{-11}$
$D=3.69 \times 10^{-13}$ 
REFERENCE CALCULATIONS: BWR REACTOR (cont'd)

Krypton 85:
$A=2.7 \times 10^{2}$
$B=\stackrel{(\# 3)}{3.91 \times 10^{2}}$
$c=2.36 \times 10^{-6}$
$D=2.82 \times 10^{-8}$

Krypton 87 :
$A=2.0 \times 10^{2}$
$B=2.84 \times 10^{7}$
$C=2.41 \times 10^{-11}$
$D=2.87 \times 10^{-13}$

Krypton 88:
$A=2.4 \times 10^{2}$
$B=\frac{(\# 5)}{1.27 \times 10^{7}}$
$C=6.47 \times 10^{-11}$
$D=7.70 \times 10^{-13}$

Krypton 89:
$A=1.0$
$B=\frac{(\# 6)}{6.60} \times 10^{8}$
$C=5.19 \times 10^{-15}$
$D=6.18 \times 10^{-17}$

9-4. Xenon 131M:

$$
A=5.0
$$

"enon 133M:

$$
A=1.0
$$

$B=\stackrel{(\# 7)}{8.45} \times 10^{4}$

$C=2.03 \times 10^{-10}$

$D=2.41 \times 10^{-12}$

Xenon 133:

$$
A=2.7 \times 10^{3}
$$

$B=4.35 \times 10^{5}$

$C=7.87 \times 10^{-12}$

$D=9.37 \times 10^{-14}$

Xenon 135M:

$$
A=7.4 \times 10^{2}
$$

$B=\stackrel{(\# 9)}{1.86 \times 10^{5}}$

$c=4.97 \times 10^{-8}$

$D=5.92 \times 10^{-10}$

Xenon 135:

$$
A=1.1 \times 10^{3}
$$$$
B=(\# 10)
$$

$C=2.84 \times 10^{-11}$

$D=3.38 \times 10^{-13}$

Xenon 137:

$$
A=1.0
$$

$$
B=2.52 \times 10^{6}
$$

$c=1.49 \times 10^{-9}$

$D=1.77 \times 10^{-11}$

Xenon 138:
$A=1.4 \times 10^{3}$
$B=8.02 \times 10^{7}$

$B=3.52 \times 10^{8}$

$c=9.73 \times 10^{-15}$

$D=1.16 \times 10^{-16}$

$c=5.98 \times 10^{-11}$

$D=7.12 \times 10^{-13}$ 
REFERENCE CALCULATIONS: BWR Reactor (cont 'd)

9-5. Iodine 131:

$$
A=4.6 \times 10^{-1} \quad B=1.24 \times 10^{5} \quad C=1.27 \times 10^{-11} \quad D=1.51 \times 10^{-13}
$$

Iodine 133:

$$
A=1.7 \quad B=1.13 \times 10^{6} \quad C=5.15 \times 10^{-12} \quad D=6.13 \times 10^{-14}
$$

9-6. Chromium 51:

$$
A=1.3 \times 10^{-2} \quad B=9.21 \times 10^{4} \quad C=4.83 \times 10^{-13} \quad D=5.75 \times 10^{-15}
$$

9-7. Manganese 54:

$$
A=4.1 \times 10^{-3} \quad B=7.98 \times 10^{3} \quad C=1.76 \times 10^{-12} \quad D=2.10 \times 10^{-14}
$$

9-8. Iron 59:

$$
A=1.1 \times 10^{-3} \quad B=4.91 \times 10^{4} \quad C=7.67 \times 10^{-14} \quad D=9.13 \times 10^{-16}
$$

9-9. Cobalt 58:

$$
\begin{aligned}
& A=1.3 \times 10^{-3} \\
& B=(\# 68) \\
& C=1.41 \times 10^{-13} \\
& D=1.68 \times 10^{-15} \\
& A=1.3 \times 10^{-2}
\end{aligned}
$$

Cobalt 60:

9-10. Zinc 65:

$$
A=2.2 \times 10^{-3} \quad B=8.25 \times 10^{3} \quad C=9.13 \times 10^{-13} \quad D=1.09 \times 10^{-14}
$$

9-11. Strontium 89:

$$
A=6.1 \times 10^{-3}
$$$$
B=2.82 \times 10^{4}
$$$$
c=7.41 \times 10^{-13}
$$$$
D=8.82 \times 10^{-15}
$$

Strontium 90:

$$
A=2.8 \times 10^{-5} \quad B=1.42 \times 10^{2} \quad C=6.75 \times 10^{-13} \quad D=8.04 \times 10^{-15}
$$


REFERENCE CALCULATIONS: BWR Reactor (cont'd)

9-12. Zirconium 95:
$A=5.0 \times 10^{-4}$
$B=2.12 \times 10^{4}$
$C=8.08 \times 10^{-14}$
$D=9.62 \times 10^{-16}$

9-13. Antimony 124:
$A=5.0 \times 10^{-4}$
$B=\frac{(\# 73)}{1.75 \times 10^{4}}$
$C=9.78 \times 10^{-14}$
$D=1.16 \times 10^{-15}$

9-14. Cesium 134:
$A=4.4 \times 10^{-3}$
$B=\frac{(\# 51)}{1.30 \times 10^{3}}$
$C=1.16 \times 10^{-11}$
$D=1.38 \times 10^{-13}$

Cesium 136:

$A=3.6 \times 10^{-4} \quad B=7.39 \times 10^{4} \quad C=1.67 \times 10^{-14} \quad D=1.99 \times 10^{-16}$

Cesium 137:
$A=6.3 \times 10^{-3}$
$B=(\# 53) \times 10^{1}$
$D=2.98 \times 10^{-12}$

9-15. Barium 140:
$A=1.1 \times 10^{-2}$
$B=\frac{(\# 55)}{7.29} \times 10^{4}$
$C=5.17 \times 10^{-13}$
$D=6.15 \times 10^{-15}$

9-16. Cerium 141:
$A=7.6 \times 10^{-4}$
$B=2.81 \times 10^{4}$
$C=9.26 \times 10^{-14}$
$D=1.10 \times 10^{-15}$

9-17. Tritium:
$A=6.8 \times 10^{1}$
$B=\stackrel{(\# 70)}{9.73} \times 10^{3}$
$C=2.39 \times 10^{-8}$
$D=2.85 \times 10^{-10}$

9-18. Carbon 14:
$A=9.5$
$B=4.46$
$C=7.29 \times 10^{-6}$
$D=8.68 \times 10^{-8}$

9-19. Sodium 24:
$A=7.5 \times 10^{-4}$
$B=8.71 \times 10^{6}$
$C=2.95 \times 10^{-16}$
$D=3.51 \times 10^{-18}$ 


\section{REFERENCE CALCULATIONS: BWR Reactor (cont'd)}

9-20. Phosphorus 32:

$$
A=3.0 \times 10^{-5} \quad B=2.85 \times 10^{5} \quad C=3.60 \times 10^{-16} \quad D=4.29 \times 10^{-18}
$$

9-21. Chromium 51:

$$
A=7.7 \times 10^{-4} \quad B=9.21 \times 10^{4} \quad C=2.86 \times 10^{-14} \quad D=3.40 \times 10^{-16}
$$

9-22. Manganese 54:

$$
A=9.1 \times 10^{-6} \quad B=7.98 \times 10^{3} \quad C=3.91 \times 10^{-15} \quad D=4.65 \times 10^{-17}
$$

Manganese 56:

$$
A=8.1 \times 10^{-4} \quad B=2.17 \times 10^{7} \quad C=1.28 \times 10^{-16} \quad D=1.52 \times 10^{-18}
$$

9-23. Iron 55:

$$
\begin{aligned}
& A=1.6 \times 10^{-4} \quad B=2.50 \times 10^{3} \quad C=2.19 \times 10^{-13} \quad D=2.61 \times 10^{-15} \\
& A=4.6 \times 10^{-6} \quad B=4.91 \times 10^{4} \quad C=3.21 \times 10^{-16} \quad D=3.82 \times 10^{-18}
\end{aligned}
$$

Iron 59:

9-24. Cobalt 58:
$A=3.1 \times 10^{-5}$
$B=3.16 \times 10^{4}$
$C=3.36 \times 10^{-15}$
$D=4.00 \times 10^{-17}$
$A=6.3 \times 10^{-5}$
$\left.B={ }^{(\# 69)}\right) \times 10^{3}$
$C=1.91 \times 10^{-13}$
$D=2.27 \times 10^{-15}$

Cobalt 60:

9-25. Nickel 65:
$A=4.6 \times 10^{-6}$
$B=\frac{(\# 78)}{1.91 \times 10^{7}}$
$C=8.25 \times 10^{-19}$
$D=9.82 \times 10^{-21}$

9-26. Copper 64:
$A=2.3 \times 10^{-3}$
$B=3.80 \times 10^{6}$
$C=2.07 \times 10^{-15}$
$D=2.46 \times 10^{-17}$ 
REFERENCE CALCULATIONS: BWR Reactor (cont'd)

9-27. Zinc 65:
$A=3.1 \times 10^{-5}$
$B=\frac{(\# 72)}{8.25 \times 10^{3}}$
$C=1.29 \times 10^{-14}$
$D=1.54 \times 10^{-16}$

Zinc 69M:
$A=1.6 \times 10^{-4}$
$B=3.27 \times 10^{6}$
$C=1.68 \times 10^{-16}$
$D=2.00 \times 10^{-18}$

Zinc 69:
$A=1.7 \times 10^{-4}$
$B=4.70 \times 10^{7}$
$c=1.24 \times 10^{-17}$
$D=1.48 \times 10^{-19}$

9-28. Wolfram 187:
$A=3.1 \times 10^{-5}$
$B=\frac{(\# 82)}{6.99} \times 10^{5}$
$C=1.52 \times 10^{-16}$
$D=1.81 \times 10^{-18}$

9-29. Neptunium 239:
$A=9.0 \times 10^{-4}$
$B=2^{(\# 83)} \times 13^{5}$
$C=1.32 \times 10^{-14}$
$D=1.57 \times 10^{-16}$

9-30. Bromine 83:
$A=4.2 \times 10^{-5}$
$B=1^{(\# 84)} \times 1.57 \times 10^{7}$
$C=9.16 \times 10^{-18}$
$D=1.09 \times 10^{-19}$

$$
A=3.4 \times 10^{-6}
$$
$B=\frac{(\# 85)}{7.04} \times 10^{7}$
$c=1.65 \times 10^{-19}$
$D=1.96 \times 10^{-21}$

Bromine 84 :

9-31. Rubidium 89:
$A=2.4 \times 10^{-5}$
$B=1.37 \times 10^{8}$
$C=6.00 \times 10^{-19}$
$D=7.14 \times 10^{-21}$

9-32. Strontium 89:
$A=1.6 \times 10^{-5}$

$B=2.82 \times 10^{4}$

$c=1.94 \times 10^{-15}$

$D=2.31 \times 10^{-17}$

Strontium 91 :

$$
A=2.5 \times 10^{-4}
$$

$B=3.56 \times 10^{6}$

$c=2.40 \times 10^{-16}$

$D=2.86 \times 10^{-18}$

Strontium 92:
$A=1.7 \times 10^{-4}$
$B=1.26 \times 10^{7}$
$c=4.62 \times 10^{-17}$
$D=5.50 \times 10^{-19}$ 
REFERENCE CALCULATIONS: BWR Reactor (cont'd)

9-33. Yttrium 91M:
$A=1.6 \times 10^{-4}$
$B=4.13 \times 10^{7}$
$C=1.33 \times 10^{-17}$
$D=1.58 \times 10^{-19}$

Yttrium 91:

$$
A=8.0 \times 10^{-6} \quad B=2.44 \times 10^{4} \quad C=1.12 \times 10^{-15} \quad D=1.34 \times 10^{-17}
$$

Yttrium 92:

$$
\begin{aligned}
& A=3.6 \times 10^{-4} \quad B=9.65 \times 10^{6} \quad C=1.28 \times 10^{-16} \quad D=1.52 \times 10^{-18} \\
& A=2.6 \times 10^{-4} \quad B=3.31 \times 10^{6} \quad C=2.69 \times 10^{-16} \quad D=3.20 \times 10^{-18}
\end{aligned}
$$

Yttrium 93:

9-34 Niobium 98:

$$
A=9.1 \times 10^{-6}
$$

$(\# 89)$

$B=3.76 \times 10^{7}$

$C=8.29 \times 10^{-19}$

$D=9.87 \times 10^{-21}$

9-35. Molybdenum 99:
$A=2.7 \times 10^{-4}$
$B=\stackrel{(\# 33)}{4.75} \times 10^{5}$
$c=1.95 \times 10^{-15}$
$D=2.32 \times 10^{-17}$

9-36. Technetium 99M:
$A=1.0 \times 10^{-3}$
$B=\frac{(\# 34)}{5.28} \times 10^{6}$
$C=6.49 \times 10^{-16}$
$D=7.73 \times 10^{-18}$

Technetium 101:

$$
A=2.3 \times 10^{-6}
$$

$B=1.33 \times 10^{8}$

$C=5.92 \times 10^{-20}$

$D=7.05 \times 10^{-22}$

Technetium 104:

$$
A=6.8 \times 10^{-6} \quad B=1.00 \times 10^{8} \quad C=2.33 \times 10^{-19} \quad D=2.77 \times 10^{-21}
$$

9-37. Ruthenium 103:

$$
A=3.4 \times 10^{-6}
$$

$B=3.20 \times 10^{4}$

$c=3.64 \times 10^{-16}$

$D=4.33 \times 10^{-18}$

Ruthenium 105:

$$
A=6.3 \times 10^{-5}
$$

$B=6.72 \times 10^{6}$

$c=3.21 \times 10^{-17}$

$D=3.82 \times 10^{-19}$ 
REFERENCE CALCULATIONS: BWR Reactor (cont'd)

9-38. Rhodium 103M:

$$
A=3.4 \times 10^{-6} \quad B=3.20 \times 10^{7} \quad C=3.64 \times 10^{-19} \quad D=4.33 \times 10^{-21}
$$

Rhodium 105M:

$$
\begin{aligned}
& A=6.4 \times 10^{-5} \quad B=2.39 \times 10^{9} \quad C=9.17 \times 10^{-20} \quad D=1.09 \times 10^{-21} \\
& A=2.1 \times 10^{-5} \quad B=8.32 \times 10^{5} \quad C=8.64 \times 10^{-17} \quad D=1.03 \times 10^{-18}
\end{aligned}
$$

Rhodium 105:

9-39. Tellurium 129M:

$$
A=5.7 \times 10^{-6} \quad B=2.98 \times 10^{4} \quad C=6.55 \times 10^{-16} \quad D=7.80 \times 10^{-18}
$$

Tellurium 129:

$$
A=3.4 \times 10^{-6} \quad B=2.11 \times 10^{7} \quad C=5.52 \times 10^{-19} \quad D=6.57 \times 10^{-21}
$$

Tellurium 131M:

$$
A=1.1 \times 10^{-5} \quad B=7.98 \times 10^{5} \quad C=4.72 \times 10^{-17} \quad D=5.62 \times 10^{-19}
$$

Tellurium 131:

$$
A=2.3 \times 10^{-6} \quad B=5.74 \times 10^{7} \quad C=1.37 \times 10^{-19} \quad D=1.63 \times 10^{-21}
$$

Tellurium 132:

$$
A=1.1 \times 10^{-6} \quad B=3.05 \times 10^{5} \quad C=1.24 \times 10^{-17} \quad 0=1.48 \times 10^{-19}
$$

9-40. Iodine 131:

$$
A=7.3 \times 10^{-4} \quad B=1.24 \times 10^{5} \quad C=2.02 \times 10^{-14} \quad D=2.40 \times 10^{-16}
$$

Iodine 132:

$$
A=3.9 \times 10^{-4}
$$

Iodine 133:

$$
A=1.9 \times 10^{-3} \quad B=1.13 \times 10^{6}
$$$$
c=1.30 \times 10^{-16}
$$$$
D=1.55 \times 10^{-18}
$$$$
C=5.76 \times 10^{-15}
$$$$
D=6.86 \times 10^{-17}
$$ 
REFERENCE CALCULATIONS: BWR Reactor (cont'd)

Iodine 134:
$A=1.6 \times 10^{-4}$
$B=\frac{(\# 94)}{2.70 \times 10^{7}}$
$C=2.03 \times 10^{-17}$
$D=2.42 \times 10^{-19}$

Iodine 135:
$A=9.0 \times 10^{-4}$
$B=3.47 \times 10^{6}$
$C=8.88 \times 10^{-16}$
$D=1.06 \times 10^{-17}$

9-41. Cesium 134:
$A=8.5 \times 10^{-4}$
$B=\frac{(\# 51)}{1.30 \times 10^{3}}$
$C=2.24 \times 10^{-12}$
$D=2.67 \times 10^{-14}$

Cesium 136:
$A=5.4 \times 10^{-4}$
$B=7^{(\# 52)} \times 1.39 \times 10^{4}$
$C=2.50 \times 10^{-14}$
$D=2.98 \times 10^{-16}$

Cesium 137:

$$
A=2.0 \times 10^{-3}
$$

$B={ }^{(\# 53)} 8.64 \times 10^{1}$

$C=7.93 \times 10^{-11}$

$D=9.44 \times 10^{-13}$

Cesium 138:
$A=8.3 \times 10^{-4}$
$B=4^{(\# 95)} \times 10^{7}$
$c=6.72 \times 10^{-17}$
$D=8.00 \times 10^{-19}$

9-42. Barium 137M:
$A=1.8 \times 10^{-3}$
$B={ }^{(\# 54)} .38 \times 10^{8}$
$c=1.15 \times 10^{-17}$
$D=1.37 \times 10^{-19}$

Barium 139:
$A=6.0 \times 10^{-5}$
$B=1.63 \times 10^{7}$
$c=1.26 \times 10^{-17}$
$D=1.50 \times 10^{-19}$

Barium 140:
$A=6.0 \times 10^{-5}$
$B=\frac{(\# 55)}{7.29 \times 10^{4}}$
$C=2.82 \times 10^{-15}$
$D=3.36 \times 10^{-17}$

9-43. Lanthanum 140:
$A=1.3 \times 10^{-5}$
$B=\frac{(\# 56)}{5.57 \times 10^{5}}$
$C=7.99 \times 10^{-17}$
$D=9.51 \times 10^{-19}$

Lanthanum 141:

$$
A=1.9 \times 10^{-5}
$$

$B=\frac{(\# 97)}{5.70 \times 10^{6}}$

$c=1.14 \times 10^{-17}$

$D=1.36 \times 10^{-19}$

Lanthanum 142:
$A=4.1 \times 10^{-5}$
$B=\frac{(\# 98)}{1.44} \times 10^{7}$
$C=9.75 \times 10^{-18}$
$D=1.16 \times 10^{-19}$ 


\section{REFERENCE CALCULATIONS: BHR Reactor (cont'd)}

9-44. Cerium 141:
$A=4.6 \times 10^{-6}$
$B=2.81 \times 10^{4}$
$C=5.61 \times 10^{-16}$
$=6.68 \times 10^{-18}$

Cerium 143:
$A=3.4 \times 10^{-6}$
$B=\frac{(\# 58)}{6.64} \times 10^{5}$
$c=1.75 \times 10^{-17}$
$D=2.08 \times 10^{-19}$

9-45. Praseodymium 143:
$A=5.7 \times 10^{-6}$
$B=6.67 \times 10^{4}$
$C=2.93 \times 10^{-16}$
$D=3.49 \times 10^{-18}$

9-46. Tritium:
$A=1.2 \times 10^{1}$
$B=\frac{(\# 70)}{9.73} \times 10^{3}$
$C=4.22 \times 10^{-9}$
$D=5.03 \times 10^{-11}$

5-16-77

(26, TABLE 3.6-1) (25, p. 11-53)

9-47. $\frac{116 \mathrm{mg} \mathrm{Ca}(\mathrm{ppm})}{\text { liter }} \times \frac{1.41 \times 10^{7} \text { liters }}{\text { day }} \times \frac{\mathrm{kg}}{10^{6} \mathrm{mg}}=1.64 \times 10^{3} \mathrm{~kg} \mathrm{Ca}$

$\therefore \frac{1.64 \times 10^{3} \mathrm{~kg} \mathrm{Ca} / \text { day }}{84 \mathrm{~kg} \mathrm{U} / \text { day }}=1.95 \times 10^{1} \frac{\mathrm{kg} \mathrm{Ca}}{\mathrm{kg} \mathrm{U}(\text { reactor input })}$

(26, TABLE 3.6-1) (25, p. 11-53
9-48. $\frac{21 \mathrm{mg} \mathrm{Mg}(\mathrm{ppm})}{1 \mathrm{iter}} \times \frac{1.41 \times 10^{7} \text { liters }}{\text { day }} \times \frac{\mathrm{kg}}{10^{6} \mathrm{mg}}=2.96 \times 10^{2} \frac{\mathrm{kg} \mathrm{Mg}}{\text { day }}$
$\therefore \frac{2.96 \times 10^{2} \mathrm{~kg} \mathrm{Mg} / \text { day }}{84 \mathrm{~kg} \mathrm{U} / \mathrm{day}}=\frac{3.52 \mathrm{~kg} \mathrm{Mg}}{\mathrm{kg} \mathrm{U}(\text { reactor input })}$ 
REFERENCE CALCULATIONS: BWR Reactor (cont'd)

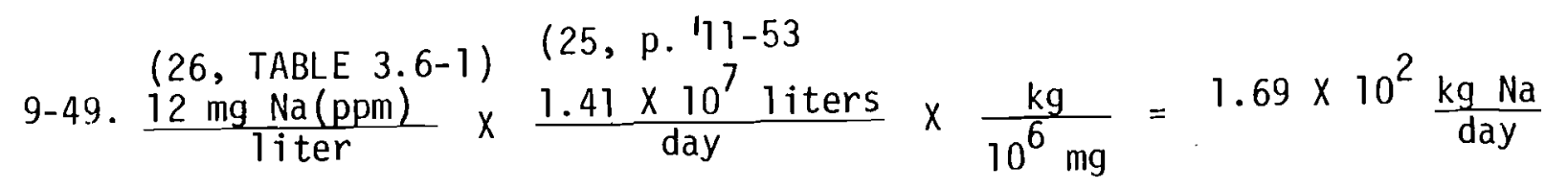

$$
\begin{aligned}
& \therefore \frac{1.69 \times 10^{2} \mathrm{~kg} \mathrm{Na} / \text { day }}{84 \mathrm{~kg} \mathrm{U} / \text { day }}=2.01 \frac{\mathrm{kg} \mathrm{Na}}{\mathrm{kg} \mathrm{U}(\text { reactor input })}
\end{aligned}
$$

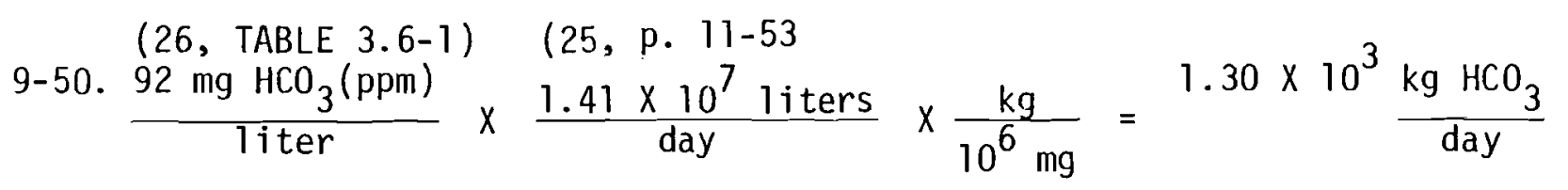

$$
\begin{aligned}
& \therefore \frac{1.30 \times 10^{3} \mathrm{HCO}_{3} / \text { day }}{84 \mathrm{~kg} \mathrm{U} / \text { day }}=\frac{1.55 \times 10^{1} \mathrm{~kg} \mathrm{HCO}_{3}}{\mathrm{kgU}(\text { reactor input })} \\
& \text { 9-51. } \frac{236 \mathrm{mg} \mathrm{S0}(\mathrm{ppm})}{1 \text { iter }} \times \frac{1.41 \times 10^{7} \text { liters }}{\text { day }} \times \frac{\mathrm{kg}}{10^{6} \mathrm{mg}}=3.33 \times 10^{3} \frac{\mathrm{kg} \mathrm{S0} 4}{\text { day }}
\end{aligned}
$$

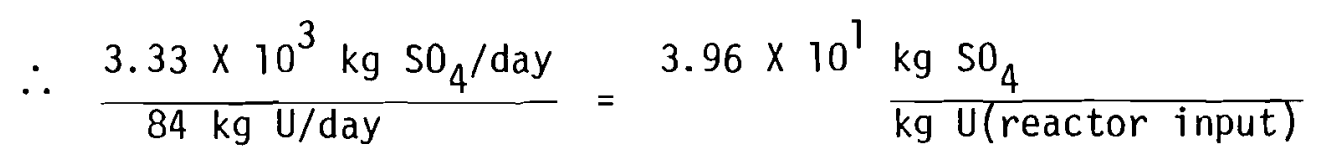

$$
\begin{aligned}
& \begin{array}{l}
\text { (26, TABLE 3.6-1) }(25, \mathrm{p}, 11-53) \\
\text { 9-52. } \frac{5 \mathrm{mg} \mathrm{Cl}(\mathrm{ppm})}{1 \text { iter }} \times \frac{1.41 \times 10^{7} \text { liters }}{\text { day }} \times \frac{\mathrm{kg}}{10^{6} \mathrm{mg}}=7.05 \times 10^{1} \frac{\mathrm{kg} \mathrm{Cl}}{\text { day }}
\end{array}
\end{aligned}
$$


$\therefore \frac{7.05 \times 10^{1} \mathrm{~kg} \mathrm{Cl} / \text { day }}{84 \mathrm{~kg} \mathrm{U} / \text { day }}=\frac{8.39 \times 10^{-1} \mathrm{~kg} \mathrm{Cl}}{\mathrm{kgU} \text { (reactor input })}$

9-53. $\frac{(26,24 \mathrm{mg} \mathrm{NOBL} 3.6-1)}{1 \text { iter }} \times \frac{(25, \mathrm{ppm})}{1.41 \times 10^{7} \text { liters }}$ day $\times \frac{\mathrm{kg}}{10^{6} \mathrm{mg}}=1.75 \times 10^{7} \frac{\mathrm{kg} \mathrm{NO}_{3}}{\text { day }}$

$\therefore \frac{1.75 \times 10^{1} \mathrm{~kg} \mathrm{NO}_{3} / \text { day }}{84 \mathrm{~kg} \mathrm{U} / \text { day }}=\frac{2.08 \times 10^{-1} \mathrm{~kg} \mathrm{NO}_{3}}{\mathrm{kgU} \text { (reactor input })}$

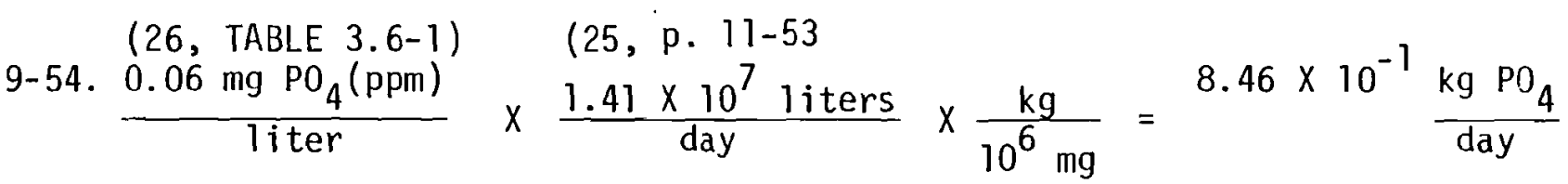

$\therefore \frac{8.46 \times 10^{-1} \mathrm{~kg} \mathrm{P0} 4 / \text { day }}{84 \mathrm{~kg} \mathrm{U} / \text { day }}=\frac{1.01 \times 10^{-2} \mathrm{~kg} \mathrm{PO}_{4}}{\text { kg U(reactor input })}$

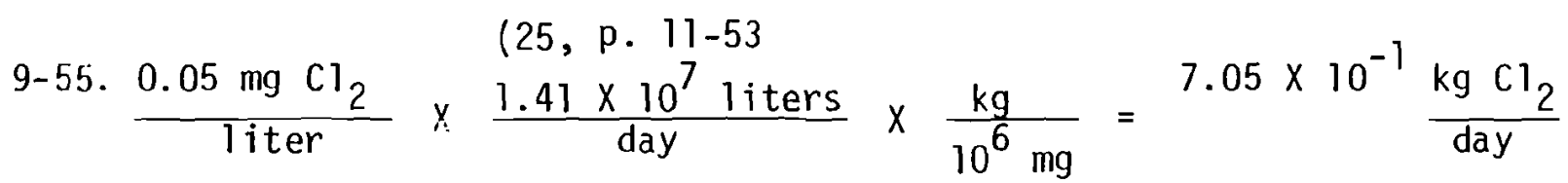

$\therefore \frac{7.05 \times 10^{-1} \mathrm{~kg} \mathrm{Cl}_{2} / \text { day }}{84 \mathrm{~kg} \mathrm{U} / \text { day }}=\frac{8.39 \times 10^{-3} \mathrm{~kg} \mathrm{Cl}_{2}}{\mathrm{~kg} \mathrm{U} \text { (reactor input) }}$ 
REFERENCE CALCULATIONS: BWR Reactor (cont'd)

$5-27-77$

9-56 Calculation of the release factors in the table below is based on the following equation:

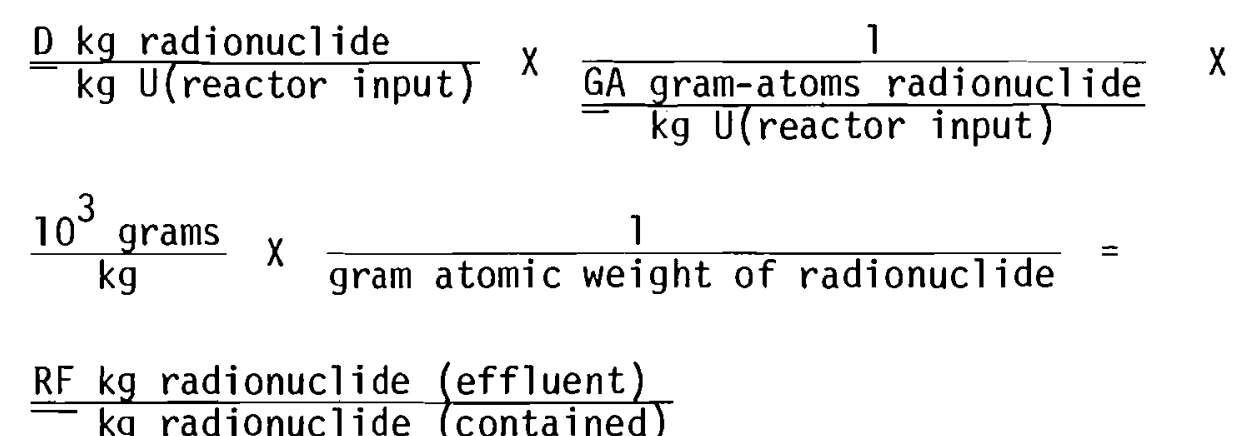

Note: D values come from reference calculations 9-2 through 9-46.

GA values come from ORIGEN code, chain 11 (for BWR at equilibrium). RF values are the release factors.

Isotope

1) Argon-41

2) Krypton-831

3) Krypton-85M

4) Krypton- 85

5) Krypton-87

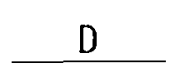

2. $44 \mathrm{E}-14$

$2.01 E-15$

3.69E-13

2. $82 \mathrm{E}-8$

2. $87 \mathrm{E}-13$

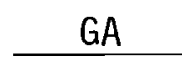

2. $59 \mathrm{E}-16$

$2.13 E-8$

$1.58 \mathrm{E}-7$

2. $67 \mathrm{E}-4$

8. $80 \mathrm{E}-8$

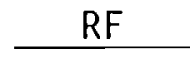

Assumed to be 1

1. 1E-6

2. $7 E-5$

1. $2 E-3$

3. $7 E-5$ 
REFERENCE CALCULATIONS: BWR Reactor (cont'd)

\begin{tabular}{|c|c|c|c|}
\hline Isotope & $D$ & $\mathrm{GA}$ & $\mathrm{RF}$ \\
\hline 6) Krypton-88 & $7.70 \mathrm{E}-13$ & $2.83 E-7$ & $3.1 E-5$ \\
\hline 7) Krypton-89 & $6.18 \mathrm{E}-17$ & $6.75 E-9$ & 1. $.0 \mathrm{E}-7$ \\
\hline 8) Xenon-131M & $2.41 E-12$ & $3.78 \mathrm{E}-7$ & $4.9 E-5$ \\
\hline 9) Xenon-133M & $9.37 E-14$ & $4.03 E-7$ & $1.7 \mathrm{E}-6$ \\
\hline 10) Xenon-133 & $5.92 \mathrm{E}-10$ & $3.89 E-5$ & $1.1 E-4$ \\
\hline 11) Xenon-135M & $3.38 \mathrm{E}-13$ & $2.14 \mathrm{E}-8$ & $1.2 \mathrm{E}-4$ \\
\hline 12) Xenon-135 & $1.77 E-11$ & $7.84 \mathrm{E}-7$ & $1.7 E-4$ \\
\hline 13) Xenon-137 & $1.16 \mathrm{E}-16$ & $1.95 \mathrm{E}-8$ & $4.3 E-8$ \\
\hline 14) Xenon-138 & $7.12 E-13$ & $8.17 E-8$ & $6.3 E-5$ \\
\hline 15) Iodine-131 & $1.51 E-13$ & $3.19 E-5$ & $3.6 \mathrm{E}-8$ \\
\hline 16) Iodine-133 & $6.13 E-14$ & $6.45 \mathrm{E}-6$ & $7.1 E-8$ \\
\hline 17) Chromium-51 & $5.75 \mathrm{E}-15$ & $4.31 E-8$ & $2.6 \mathrm{E}-6$ \\
\hline 18) Manganese-54 & $2.10 E-14$ & $1.22 \mathrm{E}-8$ & $3.2 E-5$ \\
\hline 19) Iron-59 & $9.13 \mathrm{E}-16$ & $8.04 E-10$ & $1.9 \mathrm{E}-5$ \\
\hline 20) Cobalt-58 & $1.68 \mathrm{E}-15$ & $2.01 \mathrm{E}-8$ & $1.4 \mathrm{E}-6$ \\
\hline 21) Cobalt-60 & $4.69 E-13$ & $6.27 E-9$ & $1.2 \mathrm{E}-3$ \\
\hline 22) Zinc- 65 & $1.09 E-14$ & $3.18 E-11$ & $5.3 E-3$ \\
\hline 23) Strontium-89 & $8.82 E-15$ & $1.71 \mathrm{E}-4$ & $5.8 E-10$ \\
\hline 24) Strontium-90 & $8.04 E-15$ & $4.88 E-3$ & $1.8 \mathrm{E}-11$ \\
\hline 25) Zirconium-95 & $9.62 E-16$ & $4.11 \mathrm{E}-4$ & $2.5 \mathrm{E}-11$ \\
\hline 26) Antimony-124 & $1.16 \mathrm{E}-15$ & $1.37 \mathrm{E}-7$ & $6.8 E-8$ \\
\hline
\end{tabular}


REFERENCE CALCULATIONS: BWR Reactor (cont'd)

\begin{tabular}{|c|c|c|c|}
\hline I sotope & D & $\mathrm{GA}$ & $\mathrm{RF}$ \\
\hline 27) Cesium-134 & $1.38 \mathrm{E}-13$ & $8.67 E-4$ & 1. $2 \mathrm{E}-9$ \\
\hline 28) Cesium-136 & $1.99 \mathrm{E}-16$ & $3.52 \mathrm{E}-6$ & $4.2 \mathrm{E}-10$ \\
\hline 29) Cesium-137 & $2.98 \mathrm{E}-12$ & $7.32 E-3$ & $3.0 \mathrm{E}-9$ \\
\hline 30) Barium- 140 & $6.15 \mathrm{E}-15$ & $8.52 E-5$ & $5.2 E-10$ \\
\hline 31) Cerium-141 & $1.10 \mathrm{E}-15$ & $2.04 E-4$ & $3.8 \mathrm{E}-11$ \\
\hline 32) Tritium & $2.85 \mathrm{E}-10$ & $1.3 E-5$ & $7.3 E-3$ \\
\hline 33) Carbon-14 & $8.68 E-8$ & $4.61 E-6$ & Assumed to be 1 \\
\hline 34) Sodium-24 & $3.51 E-18$ & $2.73 \mathrm{E}-12$ & $5.4 \mathrm{E}-5$ \\
\hline 35) Phosphorus-32 & $4.29 E-18$ & $8.81 E-11$ & $1.5 \mathrm{E}-6$ \\
\hline 36) Chromium-51 & $3.40 E-16$ & $4.31 E-8$ & $1.5 \mathrm{E}-7$ \\
\hline 37) Manganese-54 & $4.65 E-17$ & $1.22 E-8$ & $7.1 E-8$ \\
\hline 38) Manganese-56 & $1.52 E-18$ & $1.63 \mathrm{E}-10$ & $1.7 \mathrm{E}-7$ \\
\hline 39) Iron-55 & $2.61 E-15$ & $2.92 \mathrm{E}-7$ & $1.6 \mathrm{E}-7$ \\
\hline 40) Iron-59 & $3.82 \mathrm{E}-18$ & $8.04 \mathrm{E}-10$ & $8.1 E-8$ \\
\hline 41) Cobalt-58 & $4.00 E-17$ & $2.01 \mathrm{E}-8$ & $3.4 \mathrm{E}-8$ \\
\hline 42) Cobalt-60 & $2.27 E-15$ & $6.27 E-9$ & $6.0 \mathrm{E}-6$ \\
\hline 43) Nickel-65 & $9.82 E-21$ & $2.39 E-12$ & $6.3 E-8$ \\
\hline 44) Copper-64 & $2.46 \mathrm{E}-17$ & $1.68 \mathrm{E}-10$ & $2.3 E-6$ \\
\hline 45) Zinc-65 & $1.54 E-16$ & $3.18 \mathrm{E}-11$ & $7.5 \mathrm{E}-5$ \\
\hline 46) Zinc-69M & $2.00 E-18$ & $-0-$ & \\
\hline
\end{tabular}


REFERENCE CALCULATIONS: BWR Reactor (cont'd)

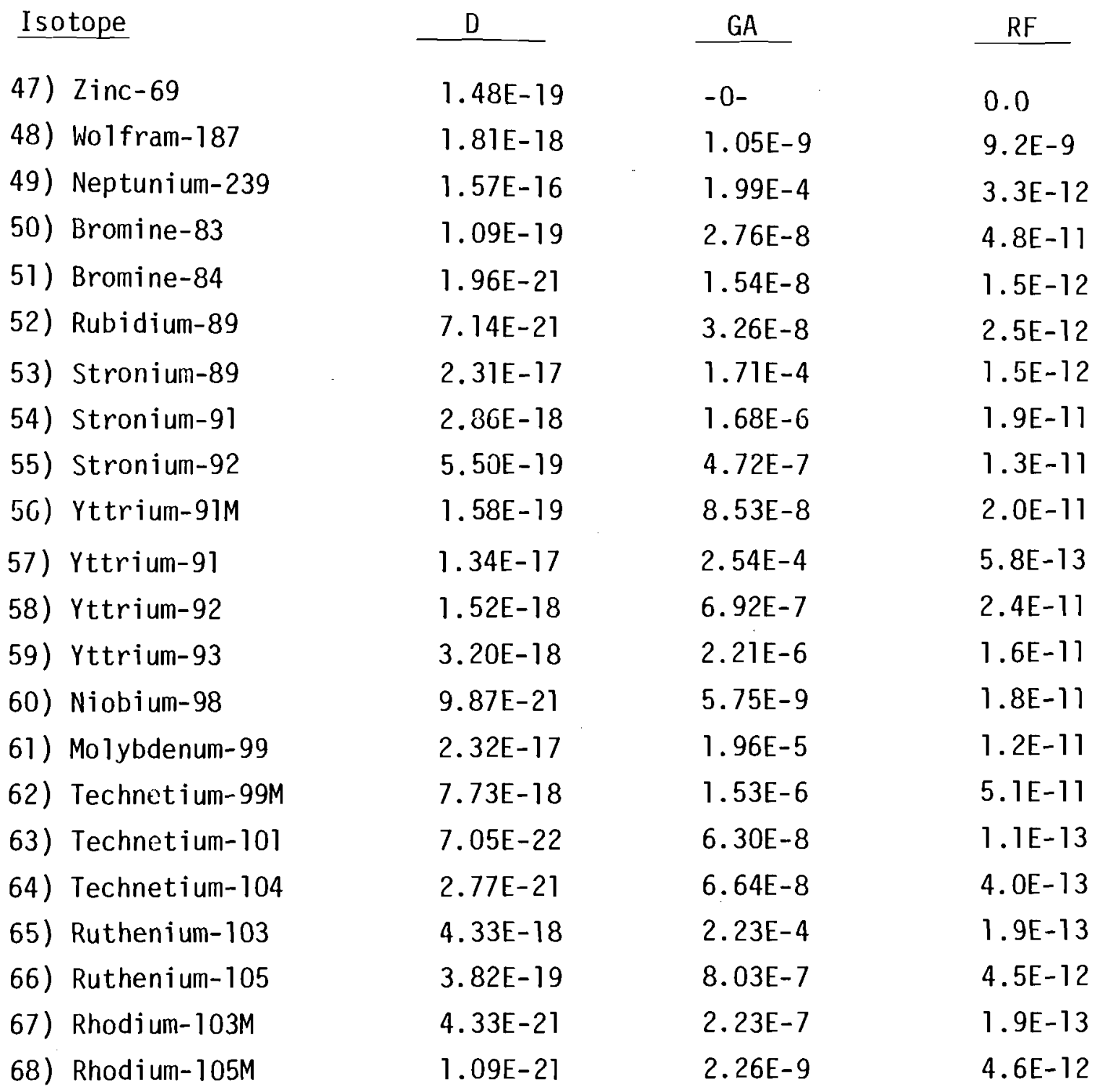


REFERENCE CALCULATIONS: BWR Reactor (cont'd)

\section{Isotope}

69) Rhodium-105

70) Tellurium-129M

71) Tellurium-129

72) Tellurium-131M

73) Tellurium-131

74) Tellurium-132

$5-31-77$

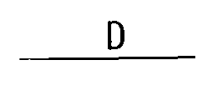

$1.03 \mathrm{E}-18$

7. $80 \mathrm{E}-18$

$6.57 \mathrm{E}-21$

$5.62 \mathrm{E}-19$

$1.63 \mathrm{E}-21$

$1.48 \mathrm{E}-19$

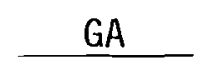

5. $20 \mathrm{E}-6$

8. $83 \mathrm{E}-6$

7. $34 \mathrm{E}-8$

7. $39 \mathrm{E}-7$

$6.03 \mathrm{E}-8$

$1.77 E-5$
RF

1.9E-12

$6.8 \mathrm{E}-12$

$6.9 \mathrm{E}-13$

$5.8 \mathrm{E}-12$

2. $1 \mathrm{E}-13$

6. $3 E-14$
75) Iodine-131
76) Iodine- 132
77) Iodine-133
78) Iodine- 134
79) Iodine-135
80) Cesium- 134
$81)$ Cesium- 136
82) Cesium-137
83) Cesium- 138

$2.40 \mathrm{E}-16$

$1.55 \mathrm{E}-18$

$6.86 \mathrm{E}-17$

2. $42 \mathrm{E}-19$

$1.06 \mathrm{E}-17$

2. $67 \mathrm{E}-14$

2. $98 \mathrm{E}-16$

9. $44 \mathrm{E}-13$

8. $00 \mathrm{E}-19$
3. $19 \mathrm{E}-5$

$5.40 \mathrm{E}-7$

6. $45 \mathrm{E}-6$

3. $07 \mathrm{E}-7$

$1.82 \mathrm{E}-6$

8. $67 \mathrm{E}-4$

3. $52 \mathrm{E}-6$

7. $32 \mathrm{E}-3$

1. $56 \mathrm{E}-7$
$5.7 \mathrm{E}-11$

2. $2 \mathrm{E}-11$

8. $0 \mathrm{E}-11$

5. $9 \mathrm{E}-12$

4. $3 \mathrm{E}-11$

2. $3 \mathrm{E}-10$

6. $2 \mathrm{E}-10$

9. $4 \mathrm{E}-10$

3. $7 \mathrm{E}-11$ 
REFERENCE CALCULATIONS: BWR Reactor (cont'd)

I so tope

84) Barium-137M

85) Barium-139

86) Barium- 140

87) Lanthanum- 140

88) Lanthanum-141

89) Lanthanum- 142

90) Cerium-141

91) Cerium- 143

92) Praseodymium- 143

93) Tritium

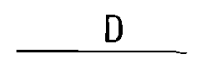

$1.37 E-19$

1. $50 \mathrm{E}-19$

3. $36 \mathrm{E}-17$

9.51E-19

1. $36 \mathrm{E}-19$

$1.16 \mathrm{E}-19$

$6.68 \mathrm{E}-18$

$2.08 \mathrm{E}-19$

$3.49 \mathrm{E}-18$

$5.03 \mathrm{E}-11$

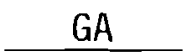

$1.11 \mathrm{E}-9$

4. $00 \mathrm{E}-7$

$8.52 E-5$

$1.15 \mathrm{E}-5$

1. $02 \mathrm{E}-6$

3. $88 \mathrm{E}-7$

2. $04 \mathrm{E}-4$

$7.70 \mathrm{E}-6$

7. $63 \mathrm{E}-5$

1. $3 \mathrm{E}-5$
RF

9. $0 \mathrm{E}-10$

2.7E-12

2. $8 \mathrm{E}-12$

$5.9 \mathrm{E}-13$

9.5E-13

2. $1 \mathrm{E}-12$

2. $3 \mathrm{E}-13$

1. $9 \mathrm{E}-13$

$3.2 \mathrm{E}-13$

1. $3 \mathrm{E}-3$ 


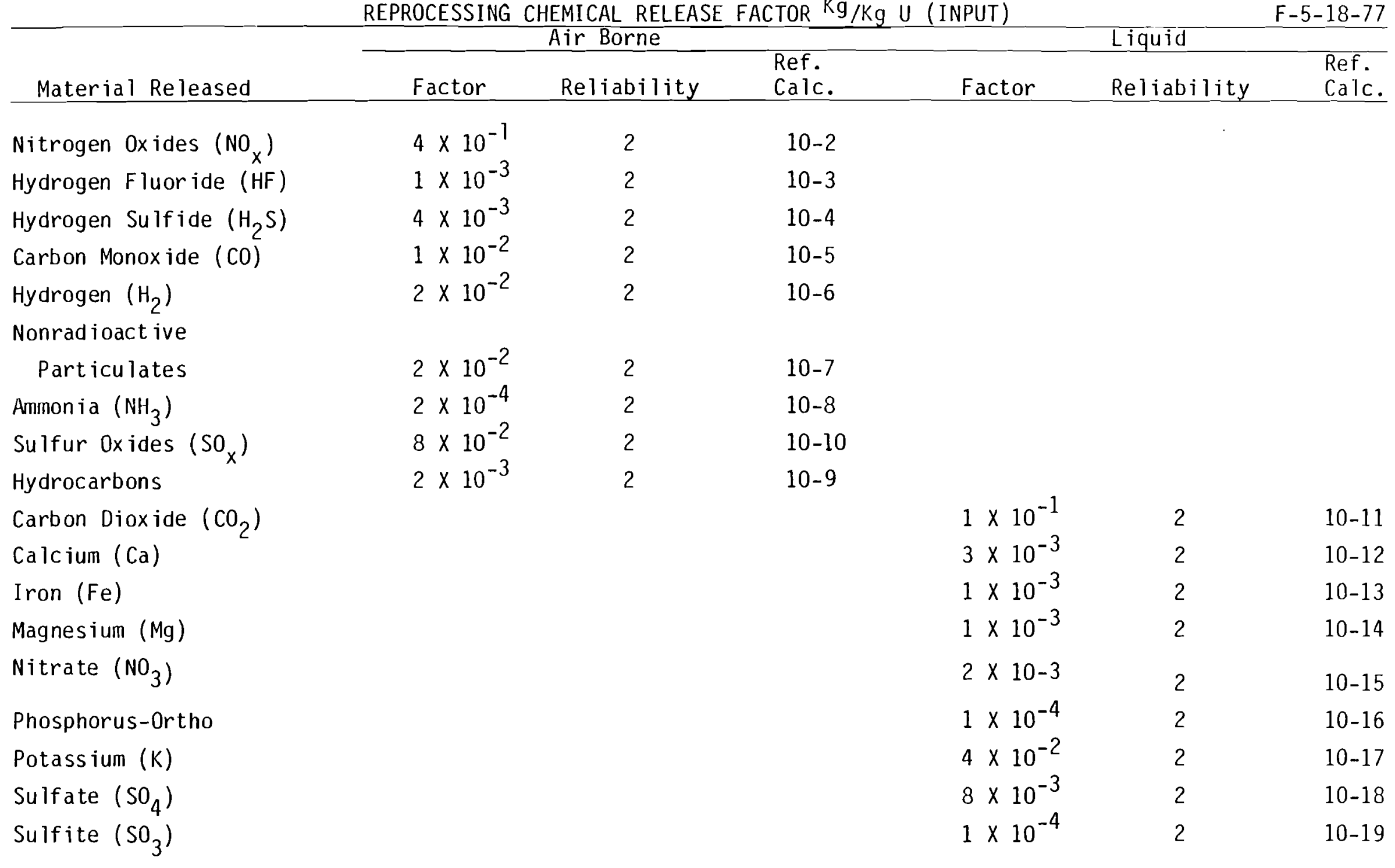




\begin{tabular}{lr}
\hline & \\
\cline { 2 - 2 } Material Released & \\
\hline & \\
Dodecane & $\mathrm{xac}$ \\
Tributyl Phosphate & $\mathrm{x}$ \\
Oxalic Acid & $\mathrm{x}$ \\
Nitrate Acid & $\mathrm{x}$ \\
Sulfuric Acid & $\mathrm{x}$ \\
Nitrogen & $\mathrm{x}$ \\
Fluorine & $\mathrm{x}$ \\
Fluorocarbon & $\mathrm{x}$ \\
Phosphoric Acid & $\mathrm{x}$
\end{tabular}
Air Borne

Acrolein

$x$ Indicates Most Likely Form of Effluent 


\begin{tabular}{|c|c|c|c|c|c|c|}
\hline \multirow[b]{2}{*}{ Material Released } & \multicolumn{2}{|c|}{ Air Borne } & \multicolumn{4}{|c|}{$\begin{array}{r}\frac{\mathrm{kg} \text { Effluent }}{\mathrm{kg} \text { Contained }} \text { OR } \frac{\mathrm{Ci} \text { Effluent }}{\mathrm{Ci} \text { Contained }} \\
\text { Liquid }\end{array}$} \\
\hline & Factor & Reliability & $\begin{array}{l}\text { Ref. } \\
\text { Calc. }\end{array}$ & Factor & Reliability & $\begin{array}{l}\text { Ref. } \\
\text { Calc. }\end{array}$ \\
\hline Tritium & $7 \times 10^{-1}$ & 2 & $10-35-01$ & & & \\
\hline \multicolumn{7}{|l|}{ Krypton } \\
\hline Krypton - 85 & 1.0 & 2 & $10-35-02$ & & & \\
\hline \multicolumn{7}{|l|}{ Stront ium } \\
\hline Strontium - 89 & $2 \times 10^{-9}$ & 2 & $10-35-03$ & & & \\
\hline Strontium - 90 & $2 \times 10^{-9}$ & 2 & $10-35-04$ & & & \\
\hline \multicolumn{7}{|l|}{ Yttrium } \\
\hline Yttrium - 90 & $2 \times 10^{-9}$ & 2 & $10-35-05$ & & & \\
\hline Yttrium - 91 & $2 \times 10^{-9}$ & 2 & $10-35-06$ & & & \\
\hline \multicolumn{7}{|l|}{ Zirconium } \\
\hline Zirconium - 95 & $3 \times 10^{-9}$ & 2 & $10-35-07$ & & & \\
\hline \multicolumn{7}{|l|}{ Niobium } \\
\hline Niobium - 95 & $3 \times 10^{-9}$ & 2 & $10-35-08$ & & & \\
\hline \multicolumn{7}{|l|}{ Ruthen ium } \\
\hline Ruthen ium - 103 & $3 \times 10^{-9}$ & 2 & $10-35-09$ & & & \\
\hline Ruthen ium - 106 & $3 \times 10^{-9}$ & 2 & $10-35-10$ & & & \\
\hline \multicolumn{7}{|l|}{ Iod ine } \\
\hline Iodine - 129 & $9 \times 10^{-4}$ & 2 & $10-35-33$ & & & \\
\hline Iodine - 131 & - $9 \times 10^{-4}$ & 2 & $10-35-33$ & & & \\
\hline \multicolumn{7}{|l|}{ Ces ium } \\
\hline Cesium - 134 & $2 \times 10^{-9}$ & 2 & $10-35-11$ & & & \\
\hline Cesium - 137 & $2 \times 10^{-9}$ & 2 & $10-35-12$ & & & \\
\hline
\end{tabular}




\begin{tabular}{|c|c|c|c|}
\hline Material Released & Factor & Reliability & $\begin{array}{l}\text { Ref. } \\
\text { Calc. }\end{array}$ \\
\hline \multicolumn{4}{|l|}{ Cer ium } \\
\hline Cerium - 141 & $3 \times 10^{-9}$ & 2 & $10-35-13$ \\
\hline Cerium - 144 & $3 \times 10^{-9}$ & 2 & $10-35-14$ \\
\hline \multicolumn{4}{|l|}{ Prometh ium } \\
\hline Promethium - 147 & $3 \times 10^{-9}$ & 2 & $10-35-15$ \\
\hline \multicolumn{4}{|l|}{ Uran ium } \\
\hline Uranium - 232 & $2 \times 10^{-5}$ & 2 & $10-35-16$ \\
\hline Uranium - 233 & $2 \times 10^{-5}$ & 2 & $10-35-17$ \\
\hline Uranium - 234 & $2 \times 10^{-5}$ & 2 & $10-35-18$ \\
\hline Uranium - 235 & $2 \times 10^{-5}$ & 2 & $10-35-19$ \\
\hline Uranium - 236 & $2 \times 10^{-5}$ & 2 & $10-35-20$ \\
\hline Uranium - 237 & $2 \times 10^{-5}$ & 2 & $10-35-21$ \\
\hline Uranium - 238 & $2 \times 10^{-5}$ & 2 & $10-35-22$ \\
\hline \multicolumn{4}{|l|}{ Plutonium } \\
\hline Plutonium - 238 & $1 \times 10^{-9}$ & 2 & $10-35-23$ \\
\hline Plutonium - 239 & $1 \times 10^{-9}$ & 2 & $10-35-24$ \\
\hline Plutonium - 240 & $1 \times 10^{-9}$ & 2 & $10-35-25$ \\
\hline Plutonium - 241 & $1 \times 10^{-9}$ & 2 & $10-35-26$ \\
\hline Plutonium - 242 & $1 \times 10^{-9}$ & 2 & $10-35-27$ \\
\hline
\end{tabular}




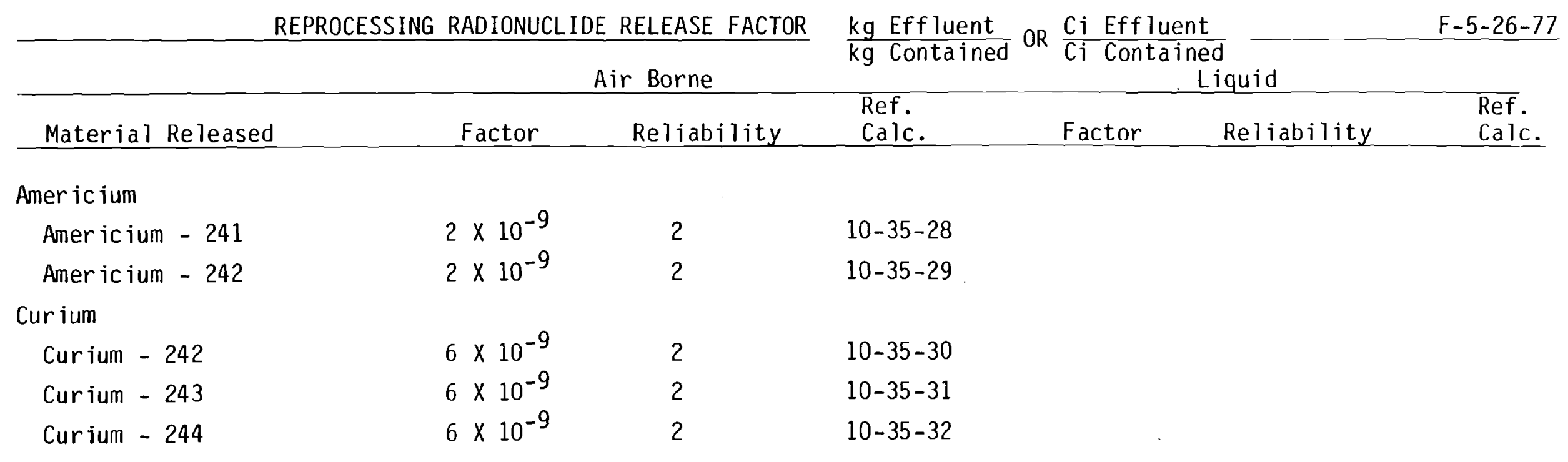




\section{REFERENCE CALCULATIONS: REPROCESSING FACILITY (300 0perating Days/yr)}

$$
5-23-77
$$

10-1. U input to reprocessing facility:

$\frac{5 \text { metric tons } U}{\text { day }}\left(\right.$ depleted $\left.0.84 \%{ }^{235} U\right) \times \frac{10^{3} \mathrm{~kg}}{\text { metric ton }}=\frac{5000 \mathrm{~kg} \mathrm{U}}{\text { day }}$

Note: (1) SF indicates main separations facility.

(2) UF6 6 indicates $U_{6} 6$ facility.

(3) PPF indicates plutonium product facility.

$\stackrel{\vec{\omega}}{\omega}$

10-2. $\left[\frac{(4 \times 24) \mathrm{mg} \mathrm{N0} x}{\mathrm{sec}}+\frac{(249}{(4 \times)}\right.$

$$
\begin{aligned}
& \text { (assumed } \mathrm{UF}_{6} \mathrm{~F} \text { ) (assumed SF) } \\
& \text { (28, App.VI p.13) (assumed PPF) } \\
& 249 \mathrm{mg} \mathrm{NO} \text { (29,p.III-5) } \\
& \frac{\sec }{\mathrm{sen}}+ \\
& \frac{22,836 \mathrm{mg} \mathrm{NO} \mathrm{x}}{\mathrm{sec}}+ \\
& 500 \mathrm{mg} \mathrm{NO}{ }_{x}
\end{aligned}
$$

$x$

$$
\frac{86,400 \mathrm{sec}}{\text { day }} \times \frac{\mathrm{kg}}{10^{6} \mathrm{mg}}=2.05 \times 10^{3} \frac{\mathrm{kg} \mathrm{NO} \times}{\text { day }}
$$

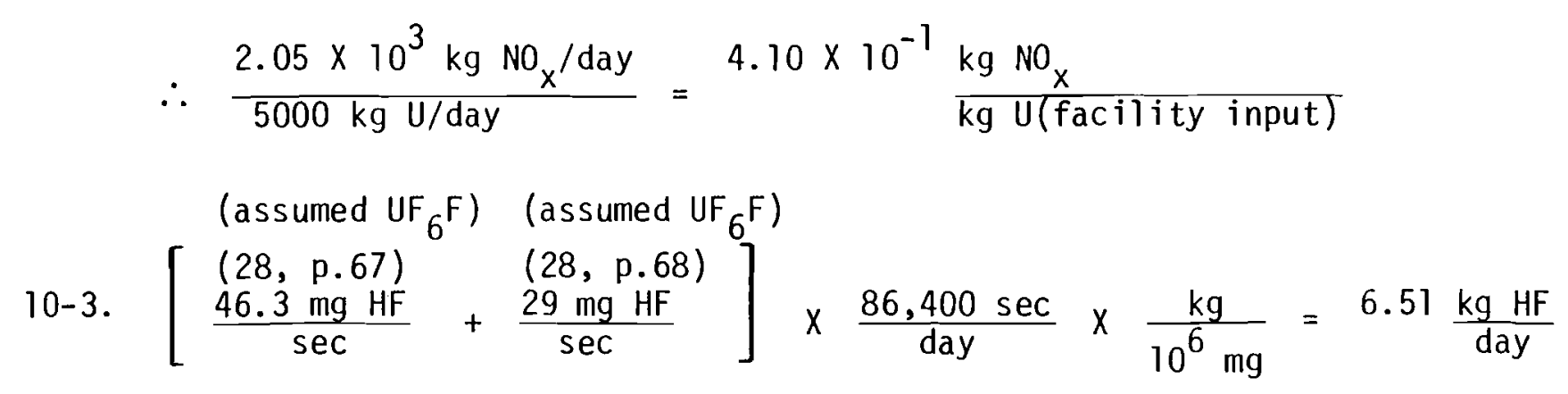

* "Input" to a reprocessing plant is a complex concept, since various fuel assemblies have received different exposures. This $5000 \mathrm{~kg} \mathrm{U} /$ day is really the weight of $U$ as charged into a reactor. The spent fuel breaks down into $\mathrm{U}, \mathrm{Pu}$ and fission products as shown on Table 12-6, page 12-50, Section 12 . 
$\therefore \frac{6.51 \mathrm{~kg} \mathrm{HF} / \text { day }}{5000 \mathrm{~kg} \mathrm{U} / \text { day }}=1.30 \times 10^{-3} \frac{\mathrm{kg} \mathrm{HF}}{\mathrm{kg} \mathrm{U}(\text { facility input })}$

(assumed $\mathrm{UF}_{6} \mathrm{~F}$ )

(28, p.68)

10-4. $249 \mathrm{mg} \mathrm{H}_{2} \mathrm{~S}$

$\frac{249 \mathrm{mg} \mathrm{H} \mathrm{H}_{2}}{\mathrm{sec}} \times \frac{86,400 \mathrm{sec}}{\text { day }} \times \frac{\mathrm{kg}}{10^{6} \mathrm{mg}}=2.15 \times 10^{1} \frac{\mathrm{kg} \mathrm{H}_{2} \mathrm{~S}}{\text { day }}$

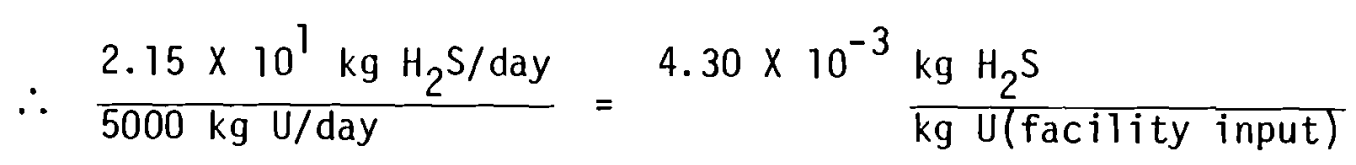

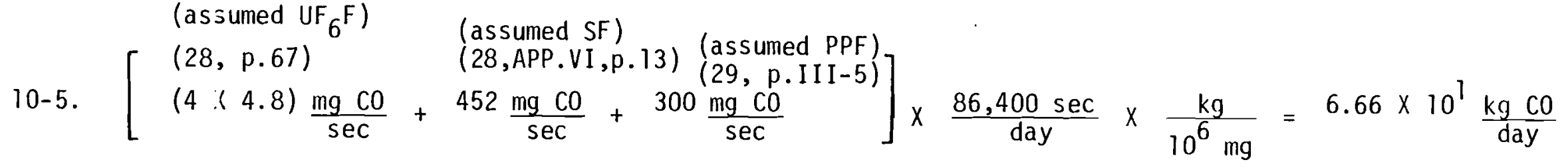

$\therefore \frac{6.66 \times 10^{1} \mathrm{~kg} \mathrm{CO} / \text { day }}{5000 \mathrm{~kg} \mathrm{U} / \mathrm{day}}=1.33 \times 10^{-2} \frac{\mathrm{kg} \mathrm{C0}}{\mathrm{kg} \mathrm{U}(\text { facility input })}$

(assumed $\mathrm{UF}_{6} \mathrm{~F}$ ) (assumed $\mathrm{UF}_{6} \mathrm{~F}$ )

10-6. $\quad\left[\begin{array}{cc}(28, \mathrm{p.} \mathrm{67)} & (28, \mathrm{p.68}) \\ 530 \mathrm{mg} \mathrm{H} & 500 \mathrm{mg} \mathrm{H_{2 }} \\ \frac{\mathrm{sec}}{\mathrm{sec}}\end{array}\right] \times \frac{86,400 \mathrm{sec}}{\text { day }} \times \frac{\mathrm{kg}}{10^{6} \mathrm{mg}}=\frac{8.90 \times 10^{1} \mathrm{~kg} \mathrm{H}}{\text { day }}$

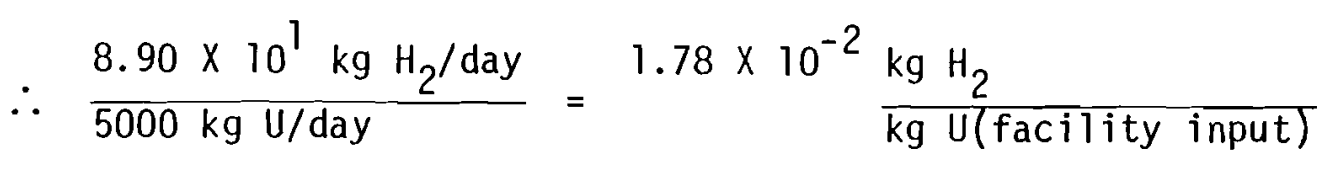


10-7. Assuming natural gas is used for heating and using EPA emission factors:

(from ref.calc. 10-5) (assumed industrial boiler)

$6.66 \times 10^{1} \frac{\mathrm{kg} \mathrm{Co}}{\text { day }} \times \frac{290 \text { nonradioactive particulate }}{270 \mathrm{C} 0}=7.15 \times 10^{1} \frac{\mathrm{kg} \text { nonradioactive particulate }}{\text { day }}$

(as sumed)

$\left(\frac{(28, \mathrm{p} .67)}{91 \mathrm{mg} \text { nonradioactive particulate }} \times \frac{86,400 \mathrm{sec}}{\text { day }} \times \frac{\mathrm{kg}}{10^{6} \mathrm{mg}}\right)+7.15 \times 10^{1} \frac{\mathrm{kg} \text { nonradioactive particulate }}{\text { day }}=$

$7.94 \times 10^{7} \frac{\mathrm{kg} \text { nonradioactive particulate }}{\text { day }}$

$\therefore \frac{7.94 \times 10^{1} \mathrm{~kg} \text { nonradioactive particulate/day }}{5000 \mathrm{~kg} \mathrm{U} / \text { day }}=1.59 \times 10^{-2} \frac{\mathrm{kg} \text { nonradioactive particulate }}{\mathrm{kg} \mathrm{U} \text { (facility input) }}$

(assumed $\mathrm{UF}_{6} \mathrm{~F}$ )

$(28$, p. 68)

10-8. $\frac{13 \mathrm{mg} \mathrm{NH}_{3}}{\mathrm{sec}} \times \frac{86,400 \mathrm{sec}}{\text { day }} \times \frac{\mathrm{kg}}{10^{6} \mathrm{mg}}=\frac{1.12 \mathrm{~kg} \mathrm{NH}_{3}}{\text { day }}$

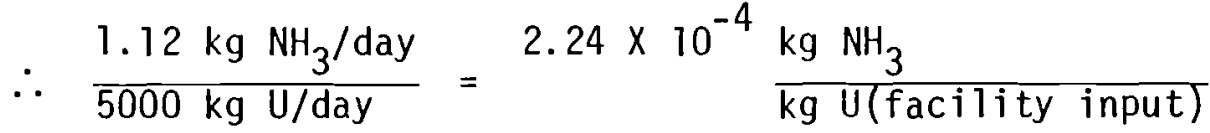

10-9. Assuming natural gas is used for heating and using EPA emission factors:

(from ref.calc. 10-5)

$6.66 \times 10^{1} \frac{\mathrm{kg} \mathrm{C0}}{\text { day }} \times \frac{48 \text { hydrocarbons }}{270 \mathrm{CO}}=1.18 \times 10^{1} \frac{\mathrm{kg} \text { hydrocarbons }}{\text { day }}$ 
REFERENCE CALCULATIONS: Reprocessing Facility (cont'd)

$\therefore \frac{1.18 \times 10^{7} \mathrm{~kg} \text { hydrocarbons } / \text { day }}{5000 \mathrm{~kg} \mathrm{U} / \mathrm{day}}=\frac{2.36 \times 10^{-3} \mathrm{~kg} \text { hydrocarbons }}{\mathrm{kg} \mathrm{U}(\text { facility input })}$

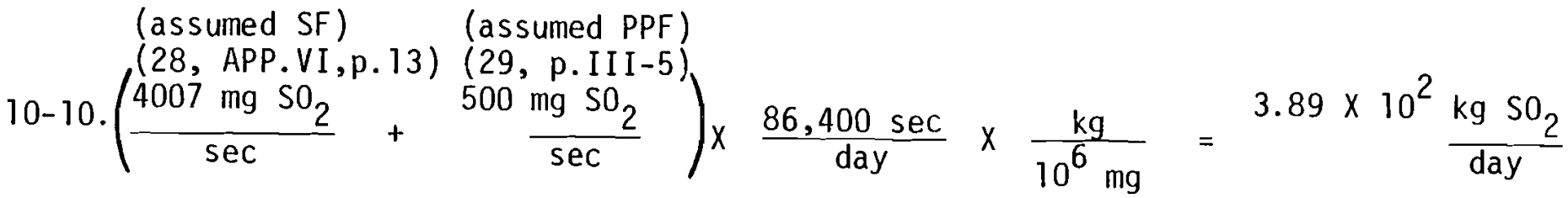

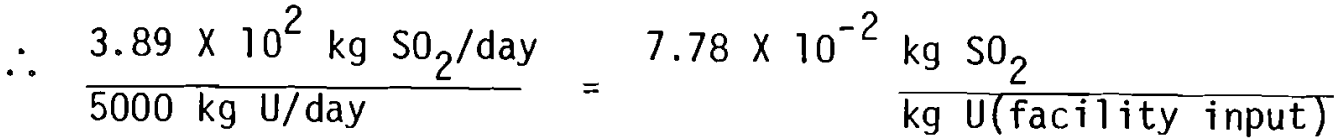

5-24-77

(assumed) (assumed)

10-11. $\frac{40 \mathrm{mg} \mathrm{CO}(\mathrm{ppm})}{\ell} \times \frac{(2311+40) \mathrm{gal}}{\min } \times \frac{1440 \mathrm{~min}}{\mathrm{day}} \times \frac{3.785 \ell}{\mathrm{ga} I} \times \frac{\mathrm{kg}}{10^{6} \mathrm{mg}}=5.12 \times 10^{2} \mathrm{~kg} \mathrm{C0} 2$

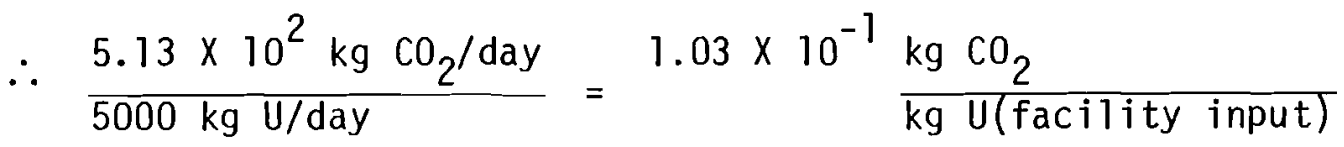

(assumed) (assumed)

10-12. $\frac{1 \mathrm{mgCa}(\mathrm{ppm})}{\ell} \times \frac{(2311+40) \mathrm{gal}}{\min } \times \frac{1440 \mathrm{~min}}{\mathrm{day}} \times \frac{3.785 \ell}{\mathrm{ga} 1} \times \frac{\mathrm{kg}}{10^{6} \mathrm{mg}}=1.28 \times 10^{1} \mathrm{~kg} \mathrm{Ca}$

$\therefore \frac{1.28 \times 10^{1} \mathrm{~kg} \mathrm{Ca} / \text { day }}{5000 \mathrm{~kg} \mathrm{U} / \mathrm{day}}=2.56 \times 10^{-3} \frac{\mathrm{kg} \mathrm{Ca}}{\mathrm{kg} \mathrm{U}(\text { facility input })}$ 
REFERENCE CALCULATIONS: Reprocessing Facility (cont'd)

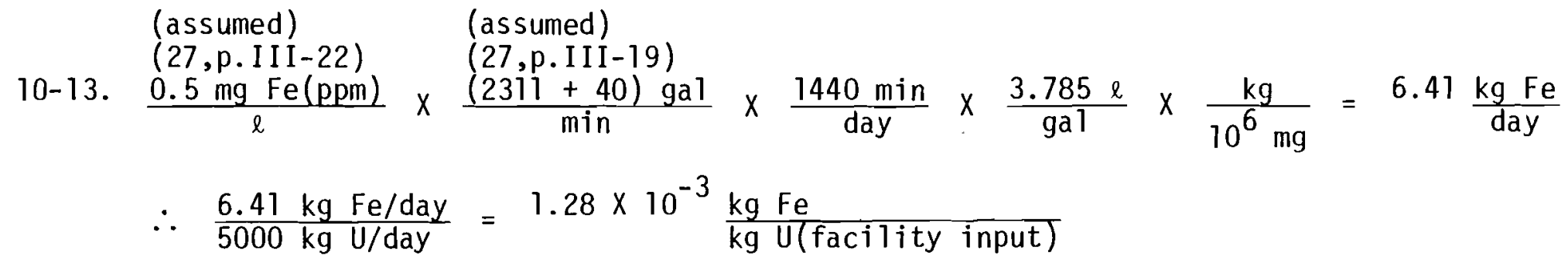

10-14. Value for Magnesium is the same as for Iron in reference calculation 10-13.

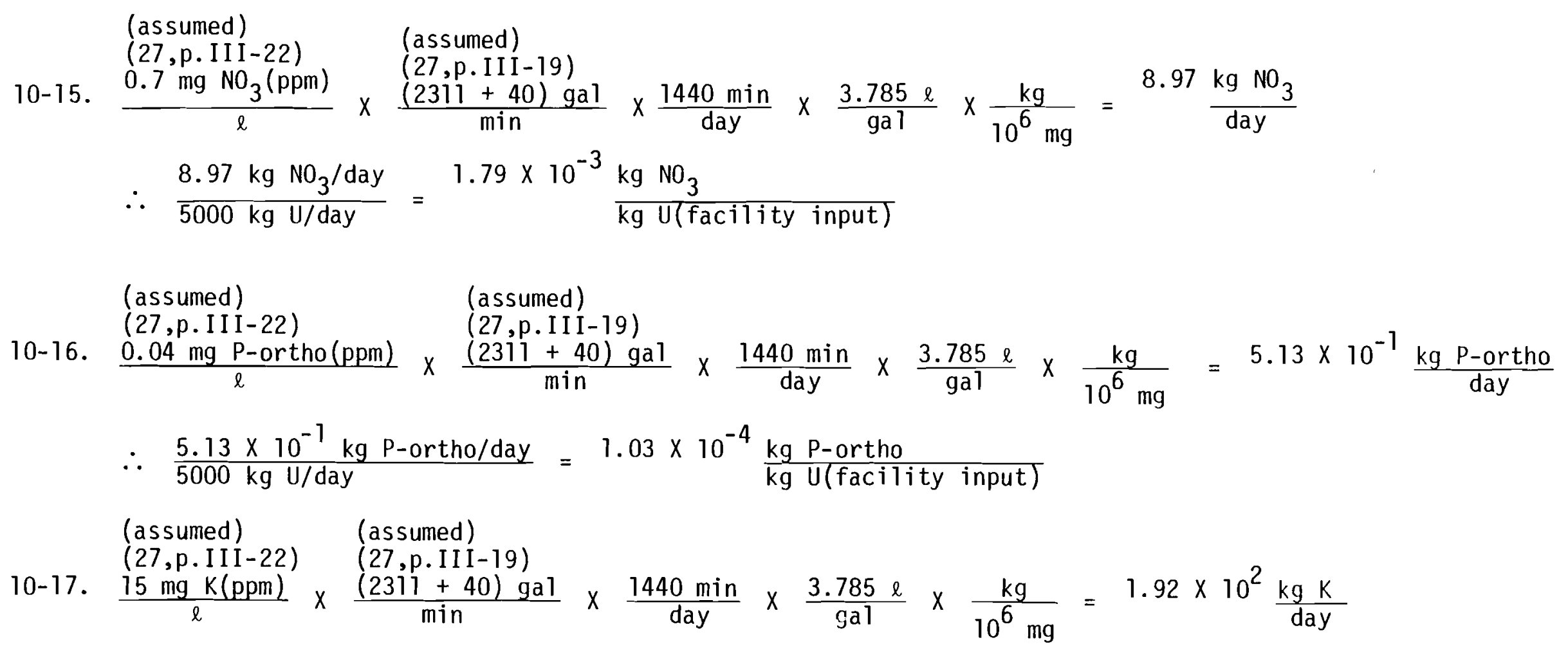


$\therefore \quad \frac{1.92 \times 10^{2} \mathrm{~kg} \mathrm{~K} / \text { day }}{5000 \mathrm{~kg} \mathrm{U} / \text { day }}=3.84 \times 10^{-2} \frac{\mathrm{kg} \mathrm{K}}{\mathrm{kg} \mathrm{U} \text { (facility input })}$

(assumed)

(27,p.I I I-22) (assumed)

10-18. $\frac{3 \mathrm{mg} \mathrm{SO}(\mathrm{ppm})}{\ell} \times \frac{(2311+40) \mathrm{gal}}{\mathrm{min}} \times \frac{1440 \mathrm{~min}}{\text { day }} \times \frac{3.785 \ell}{\mathrm{gal}} \times \frac{\mathrm{kg}}{10^{6} \mathrm{mg}}=3.84 \times 10^{1} \mathrm{~kg} \mathrm{S0} 4$

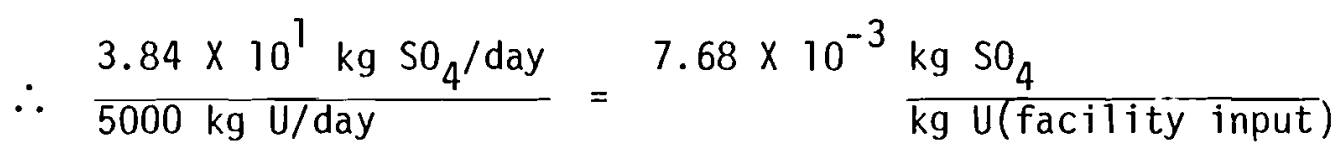

$$
\begin{aligned}
& \text { (assumed) (assumed) }
\end{aligned}
$$

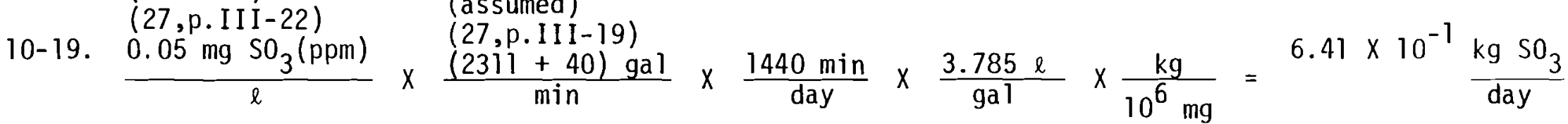

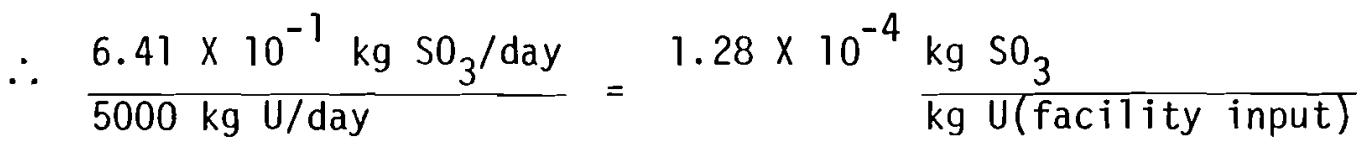

Reference calculations 10-20 through 10-33 use the following format for calculation of numbers:

$\frac{A C i \text { radionuclide }}{\mathrm{sec}} \times \frac{86,400 \mathrm{sec}}{\text { day }} \times \frac{\text { g radionuclide }}{\underline{B} \text { Ci radionuclide }} \times \frac{\mathrm{kg}}{10^{3} \mathrm{~g}}=\frac{\mathrm{C} \mathrm{kg} \text { radionuclide }}{\text { operating day }}$

C $\mathrm{kg}$ radionuclide/operating day $=$ D $\mathrm{kg}$ radionuclide

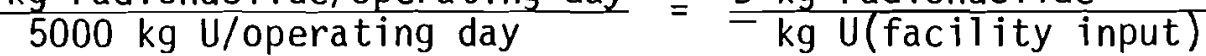




\section{REFERENCE CALCULATIONS: Reprocessing Facility (cont'd)}

10-20. Tritium:

$$
\stackrel{(28, A p p . V I, p .9 S F)}{=} 1.8 \times 10^{-2} \quad B=9.73^{(30, \# 1)} \times 10^{3} \quad C=1.60 \times 10^{-4} \quad D=3.20 \times 10^{-8}
$$

10-21. Krypton 85:

$$
\begin{aligned}
& \text { (28,App.VI,p.9 SF) } \\
& A=4.3 \times 10^{-1} \quad B=3.91 \times 10^{2} \quad C=9.50 \times 10^{-2} \quad D=1.90 \times 10^{-5}
\end{aligned}
$$

10-22. Strontium 89:

$$
\begin{aligned}
& (28, \text { App.VI,p.9 SF }) \\
& A=7.6 \times 10^{-9} \quad B=2.82 \times 10^{4} \quad C=2.33 \times 10^{-11} \quad D=4.66 \times 10^{-15}
\end{aligned}
$$

Strontium 90:

$$
\begin{aligned}
& (28, \text { App.VI, p. } 9,10,11 \text { SF }) \\
& A=\left(8.8 \times 10^{-9}+5 \times 10^{-11}+3 \times 10^{-16}\right) \quad B=1.42 \times 10^{2} \\
& C=5.38 \times 10^{-9} \quad D=1.08 \times 10^{-12}
\end{aligned}
$$

10-23. Yttrium 90:

$$
\begin{aligned}
& (28, \text { App.VI,p.9 SF }) \\
& A=8.8 \times 10^{-9} \quad B=5.44 \times 10^{5} \quad C=1.40 \times 10^{-12} \quad D=2.80 \times 10^{-16}
\end{aligned}
$$

Yttrium 91:

$$
\begin{aligned}
& (28, \text { App.VI, p.9 SF }) \\
& A=1.6 \times 10^{-8} \quad B=2.44 \times 10^{4} \quad C=5.67 \times 10^{-11} \quad D=1.13 \times 10^{-14}
\end{aligned}
$$


REFERENCE CALCULATIONS: Reprocessing Facility (cont'd)

10-24. Zirconium 95:

$$
\begin{gathered}
A=\left[\frac{2.8 \times 10^{-8} \mathrm{Ci}}{\mathrm{sec}}+\left(1.1 \times 10^{-3} \frac{\mathrm{Ci}}{\mathrm{yr}}+1.5 \times 10^{-5} \frac{\mathrm{Cj}}{\mathrm{yr}}+4.8 \times 10^{-4} \frac{\mathrm{C} j}{\mathrm{yr}}\right) \times \frac{\mathrm{yr}}{300 \text { days }} \times \frac{\text { day }}{86,400 \mathrm{sec}}\right] \\
B=2.12 \times 10^{4} \quad C=1.14 \times 10^{-10} \quad \mathrm{D}=2.28 \times 10^{-14}
\end{gathered}
$$

10-25. Niobium 95:

$$
\begin{aligned}
& (28, A p p . V I, p .9 \text { SF }) \\
& A=5.3 \times 10^{-8} \quad B=3.91^{(30, \# 9)} \times 10^{4} \quad C=1.17 \times 10^{-10} \quad D=2.34 \times 10^{-14}
\end{aligned}
$$

10-26. Ruthenium 103:

$$
\begin{aligned}
& (28, \text { App.VI,p.9 SF }) \\
& A=8.4 \times 10^{-9} \quad B=3.20 \times 10^{4} \quad C=2.27 \times 10^{-11} \quad D=4.54 \times 10^{-15}
\end{aligned}
$$

Ruthenium 106:

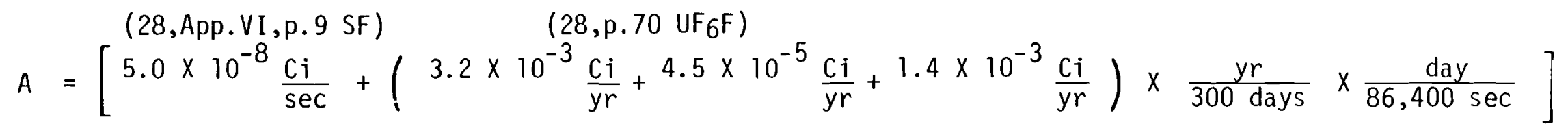

$$
\begin{aligned}
& B=3.36 \times 10^{3} \quad C=1.29 \times 10^{-9} \quad D=2.58 \times 10^{-13}
\end{aligned}
$$

10-27. Cesium 134:

(28,App.VI, p. 9,10,11 SF)

$A=\left(1.9 \times 10^{-8}+1 \times 10^{-10}+6 \times 10^{-16}\right)$

$$
C=1.27 \times 10^{-9} \quad D=2.54 \times 10^{-13}
$$


REFERENCE CALCULATIONS: Reprocessing Facility (cont'd)

Cesium 137:

$(28$, App.VI, p.9,10,11 SF $)$
$A=\left(1.2 \times 10^{-8}+6 \times 10^{-11}+4 \times 10^{-16}\right) \quad B=8.64 \times 10^{1} \cdot C=1.21 \times 10^{-8} \quad 0=2.42 \times 10^{-12}$

10-28. Cerium 141:

(28, App.VI, p.9 SF)
$A=5.5 \times 10^{-9} \quad B=2.81 \times 10^{4} \quad C=1.69 \times 10^{-11} \quad D=3.38 \times 10^{-15}$

Cerium 144:
(28, App.VI, p.9 SF)
$A=8.4 \times 10^{-8} B=3.18 \times 10^{3}$
$C=2.28 \times 10^{-9}$
$D=4.56 \times 10^{-13}$

10-29. Promethium 147:
(28, App.VI, p. 9 SF)
$A=1.5 \times 10^{-8} \quad B=9.74 \times 10^{2}$
$C=1.33 \times 10^{-9}$
$D=2.66 \times 10^{-13}$

10-30. Uranium 232:

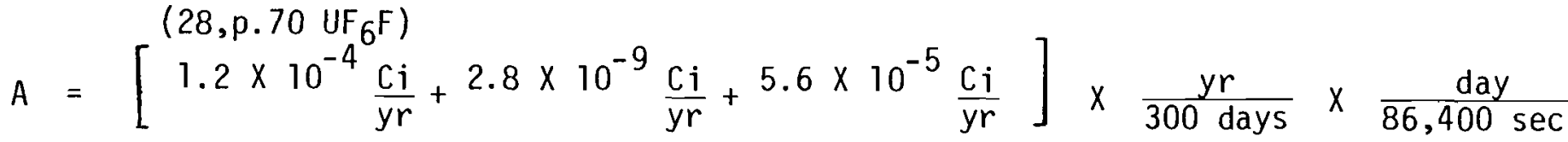

$$
\begin{aligned}
& B=2.10 \times 10^{1} \quad C=2.79 \times 10^{-11} \quad D=5.58 \times 10^{-15}
\end{aligned}
$$

Uranium 233:

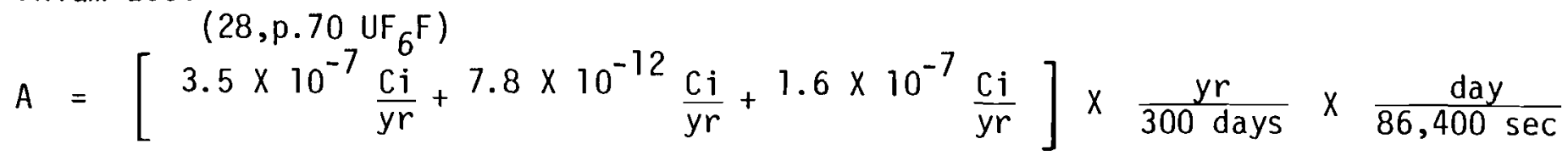


REFERENCE CALCULATIONS: Reprocessing Facility (cont'd)

$$
B=9.18 \times 10^{-3} \quad C=1.79 \times 10^{-10} \quad D=3.58 \times 10^{-14}
$$

Uranium 234 :

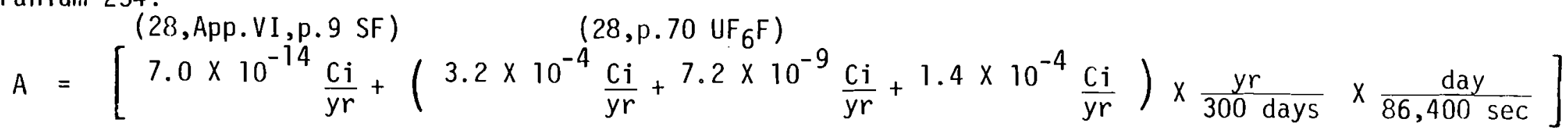

$$
\begin{aligned}
& B=6.19 \times 10^{-3} \quad C=2.49 \times 10^{-7} \quad D=4.98 \times 10^{-11}
\end{aligned}
$$

Uranium 235:

$$
\begin{aligned}
& A=\left[\begin{array}{l}
(28, A p p . V I, p .9 \text { SF }) \\
\left.7.0 \times 10^{-16} \frac{\mathrm{Ci}}{\mathrm{yr}}+\left(3.6 \times 10^{-6} \frac{\mathrm{Ci}}{\mathrm{yr}}+8.2 \times 10^{-11} \frac{\mathrm{Ci}}{\mathrm{yr}}+1.6 \times 10^{-5} \frac{\mathrm{C} i}{\mathrm{yr}}\right) \times \frac{\mathrm{yr}}{300 \mathrm{days}} \times \frac{\mathrm{day}}{86,400 \mathrm{sec}}\right]
\end{array}\right. \\
& B=2.14 \times 10^{-6} \quad C=3.06 \times 10^{-5} \quad D=6.12 \times 10^{-9}
\end{aligned}
$$

Uranium 236:

$$
\begin{aligned}
& A=\left[5.5 \times 10^{-3} \frac{\mathrm{ci}}{\mathrm{yr}}+1.2 \times 10^{-7} \frac{\mathrm{ci}}{\mathrm{yr}}+2.5 \times 10^{-3} \frac{\mathrm{Ci}}{\mathrm{yr}}\right] \times \frac{\mathrm{yr}}{300 \text { days }} \times \frac{\mathrm{day}}{86,400 \mathrm{sec}} \\
& B=6.35 \times 10^{-5} \quad C=4.20 \times 10^{-4} \quad D=8.40 \times 10^{-8}
\end{aligned}
$$

Uranium 237 :

$$
\begin{aligned}
& A=\left[6.3 \times 10^{-2} \frac{\mathrm{ci}}{\mathrm{yr}}+1.4 \times 10^{-6} \frac{\mathrm{Cj}}{\mathrm{yr}}+2.9 \times 10^{-2} \frac{\mathrm{Cj}}{\mathrm{yr}}\right] \times \frac{\mathrm{yr}}{300 \text { days }} \times \frac{\text { day }}{86,400 \mathrm{sec}} \\
& B=8.17 \times 10^{4} \quad C=3.75 \times 10^{-12} \quad D=7.50 \times 10^{-16}
\end{aligned}
$$




\section{REFERENCE CALCULATIONS: Reprocessing Facility (cont'd)}

Uranium 238:

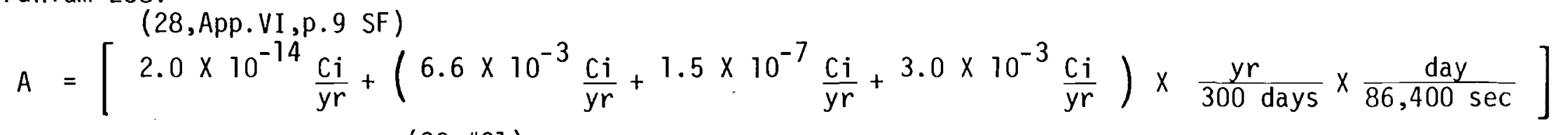

$$
\begin{aligned}
& B=3.33 \times 10^{-7} \quad C=9.61 \times 10^{-2} \quad D=1.92 \times 10^{-5}
\end{aligned}
$$

10-31. The basis for plutonium calculations for the plutonium product facility is the isotopic distribution as follows from reference $31, \mathrm{p} .10-13$ :

$\begin{array}{lr}\text { plutonium-238 } & 2.5 \% \\ \text { plutonium-239 } & 50.0 \% \\ \text { plutonium-240 } & 25.0 \% \\ \text { plutonium-241 } & 15.0 \% \\ \text { plutonium-242 } & 7.5 \%\end{array}$

and the release rate of $1.55 \times 10^{-13}$ grams of plutonium per second (Ref. 31, p. 10-12).

$$
\begin{aligned}
& \text { Plutonium 238: } \quad \text { (28,App.VI, p.9,10,11 SF ) } \quad\left(28, p .70 \mathrm{UF}_{6} \mathrm{~F}\right) \\
& A=\left[\left(1.3 \times 10^{-10} \frac{\mathrm{Ci}}{\mathrm{sec}}+4 \times 10^{-11} \frac{\mathrm{Ci}}{\mathrm{sec}}+1 \times 10^{-15} \frac{\mathrm{Ci}}{\mathrm{sec}}\right)+\left(9.1 \times 10^{-5} \frac{\mathrm{Ci}}{\mathrm{yr}}+5.2 \times 10^{-7} \frac{\mathrm{Cj}}{\mathrm{yr}}+\right.\right. \\
& \text { (see above PPF) } \\
& \left.\left.4.1 \times 10^{-5} \frac{\mathrm{Ci}}{\mathrm{yr}}\right) \times \frac{\mathrm{yr}}{300 \mathrm{days}} \times \frac{\mathrm{day}}{86,400 \mathrm{sec}}+\left(1.55 \times 10^{-13} \frac{\mathrm{g}}{\mathrm{sec}} \times 0.025 \times 1.75 \times 10^{1} \frac{\mathrm{C} i}{\mathrm{~g}}\right)\right] \\
& B={ }^{(30,732)} \times 10^{1} \quad C=8.65 \times 10^{-10} \quad D=1.73 \times 10^{-13}
\end{aligned}
$$




\section{REFERENCE CALCULATIONS: Reprocessing Facility (cont'd)}

\section{Plutonium 239:}

$$
\begin{aligned}
& A=\left[\left(1.2 \times 10^{-11} \frac{\mathrm{Ci}}{\mathrm{sec}}+4 \times 10^{-12} \frac{\mathrm{Cj}}{\mathrm{sec}}+1 \times 10^{-16} \frac{\mathrm{Ci}}{\mathrm{sec}}\right)+\left(6.6 \times 10^{-6} \frac{\mathrm{Ci}}{\mathrm{yr}}+3.7 \times 10^{-8} \frac{\mathrm{Ci}}{\mathrm{yr}}+\right.\right. \\
& \text { (see above PPF) (30,\#33) } \\
& \left.\left.3.0 \times 10^{-6} \frac{\mathrm{Cj}}{\mathrm{yr}}\right) \times \frac{\mathrm{yr}}{300 \text { days }} \times \frac{\mathrm{day}}{86,400 \mathrm{sec}}+\left(1.55 \times 10^{-13} \frac{\mathrm{g}}{\mathrm{sec}} \times 0.5 \times 6.14 \times 10^{-2} \frac{\mathrm{Ci}}{\mathrm{yr}}\right)\right] \\
& (30, \# 33) \\
& B=6.14 \times 10^{-2} \quad C=2.30 \times 10^{-8} \quad D=4.60 \times 10^{-12}
\end{aligned}
$$

Plutonium 240:

$$
\begin{aligned}
& A=\left[\left(2.1 \times 10^{-11} \frac{\mathrm{Cj}}{\mathrm{sec}}+6 \times 10^{-12} \frac{\mathrm{Cj}}{\mathrm{sec}}+2 \times 10^{-16} \frac{\mathrm{Cj}}{\mathrm{sec}}\right)+\left(1.2 \times 10^{-5} \frac{\mathrm{Ci}}{\mathrm{yr}}+6.8 \times 10^{-8} \frac{\mathrm{Ci}}{\mathrm{yr}}+\right.\right. \\
& \text { (see above PPF) } \quad(30, \# 34) \\
& \left.\left.5.5 \times 10^{-6} \frac{\mathrm{Ci}}{\mathrm{yr}}\right) \times \frac{\mathrm{yr}}{300 \text { days }} \times \frac{\text { day }}{86,400 \mathrm{sec}}+\left(1.55 \times 10^{-13} \frac{\mathrm{g}}{\mathrm{sec}} \times 0.25 \times 2.27 \times 10^{-1} \frac{\mathrm{Ci}}{\mathrm{g}}\right)\right] \\
& B=2.27 \times 10^{-1} \quad C=1.05 \times 10^{-8} \quad D=2.10 \times 10^{-12}
\end{aligned}
$$

Plutonium 241:

$$
A=\left[\left(5.5 \times 10^{-9} \frac{\mathrm{Ci}}{\mathrm{sec}}+2 \times 10^{-9} \frac{\mathrm{Ci}}{\mathrm{sec}}+5 \times 10^{-14} \frac{\mathrm{Ci}}{\mathrm{sec}}\right)+\left(3.2 \times 10^{-3} \frac{\mathrm{Ci}}{\mathrm{yr}}+1.8 \times 10^{-5} \frac{\mathrm{Ci}}{\mathrm{yr}}+\right.\right.
$$


REFERENCE CALCULATIONS: Reprocessing Facility (cont'd)

$$
\begin{aligned}
& \left.1.4 \times 10^{-3} \frac{\mathrm{Ci}}{\mathrm{yr}}\right) \times \frac{\mathrm{yr}}{300 \mathrm{days}} \times \frac{\mathrm{day}}{86,400 \mathrm{sec}}+\left(\begin{array}{cc}
\text { (see above PPF) } & (30, \# 35) \\
1.55 \times 10^{-13} & \left.\left.\frac{\mathrm{g}}{\mathrm{sec}} \times 0.15 \times 1.13 \times 10^{2} \frac{\mathrm{Ci}}{\mathrm{g}}\right)\right]
\end{array}\right. \\
& B=1.13 \times 10^{2} \quad C=5.87 \times 10^{-9} \quad D=1.17 \times 10^{-12}
\end{aligned}
$$

Plutonium 242:

$$
\begin{aligned}
& (28, \text { App.VI,p.9 SF }) \quad\left(28, \text { p. } 70 \mathrm{UF}_{6} \mathrm{~F}\right) \\
& A=\left[1.1 \times 10^{-13} \frac{\mathrm{Ci}}{\mathrm{sec}}+\left(5.6 \times 10^{-8} \frac{\mathrm{Ci}}{\mathrm{yr}}+3.4 \times 10^{-10} \frac{\mathrm{Ci}}{\mathrm{yr}}+2.8 \times 10^{-8} \frac{\mathrm{Ci}}{\mathrm{yr}}\right) \times\right. \\
& \left.\frac{\mathrm{yr}}{300 \mathrm{days}} \times \frac{\mathrm{day}}{86,400 \mathrm{sec}}+\left(1.55 \times 10^{-13} \frac{\mathrm{g}}{\mathrm{sec}} \times 0.075 \times 3.90 \times 10^{-3} \frac{\mathrm{Ci}}{\mathrm{g}}\right)\right] \\
& B=3.90 \times 10^{-3} \quad C=2.51 \times 10^{-9} \quad D=5.02 \times 10^{-13}
\end{aligned}
$$

10-32. Americium 241:

$$
\begin{array}{r}
A=\left[\left(3.2 \times 10^{-11} \frac{\mathrm{Ci}}{\mathrm{sec}}+2 \times 10^{-13} \frac{\mathrm{Ci}}{\mathrm{sec}}+3 \times 10^{-16} \frac{\mathrm{Ci}}{\mathrm{sec}}\right)\right] \\
B=(30, \# 37) \\
B=3.24 \quad C=8.59 \times 10^{-10} \quad D=1.72 \times 10^{-13}
\end{array}
$$

Americium 242:

$$
A=6.3 \times 10^{-13} \quad B=8.1 \times 10^{(28, A p p . V I, p .9 S F} \quad C=6.72 \times 10^{-17} \quad D=1.34 \times 10^{-20}
$$


REFERENCE CALCULATIONS: Reprocessing Facility (cont'd)

10-33. Curium 242:

$$
\begin{aligned}
& \text { (28,App.VI,p.9,10,11 SF) } \\
& =\left[\left(3.7 \times 10^{-9} \frac{\mathrm{Ci}}{\mathrm{sec}}+2 \times 10^{-11} \frac{\mathrm{Ci}}{\mathrm{sec}}+1 \times 10^{-16} \frac{\mathrm{Ci}}{\mathrm{sec}}\right)\right] \\
& B=3.31 \times 10^{3} \quad C=9.71 \times 10^{-11} \quad \mathrm{C}=1.94 \times 10^{-14}
\end{aligned}
$$

Curium 243:

$$
A=2.5 \times 10^{-12} \quad B=4.60 \times 10^{(28, A p p \cdot V I, p .9 S F)} \quad C=4.70 \times 10^{-12} \quad D=9.40 \times 10^{-16}
$$

$\stackrel{\text { N }}{\text { N }}$

Curium 244:

$$
\begin{aligned}
& A=\left[\left(3.9 \times 10^{-10} \frac{\mathrm{Ci}}{\mathrm{sec}}+2 \times 10^{-12} \frac{\mathrm{Ci}}{\mathrm{sec}}+1 \times 10^{-17} \frac{\mathrm{Ci}}{\mathrm{sec}}\right)\right] \\
& B=8.33^{(30, \# 42)} \times 10^{1} \quad C=4.07 \times 10^{-10} \quad D=8.14 \times 10^{-14}
\end{aligned}
$$

10-34. Iodine - Derived from Iodine 129 because of its long radioactive half 1 ife.

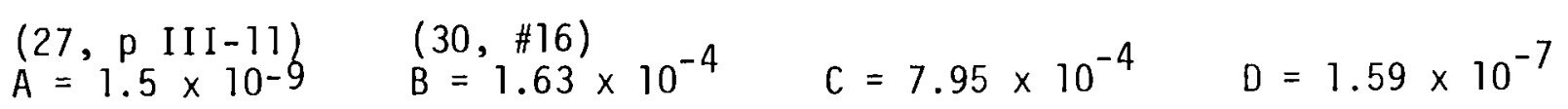


REFERENCE CALCULATIONS: Reprocessing Facility (cont'd)

$5-31-77$

10-35. Calculation of the release factors in the table below is based on the following equation:

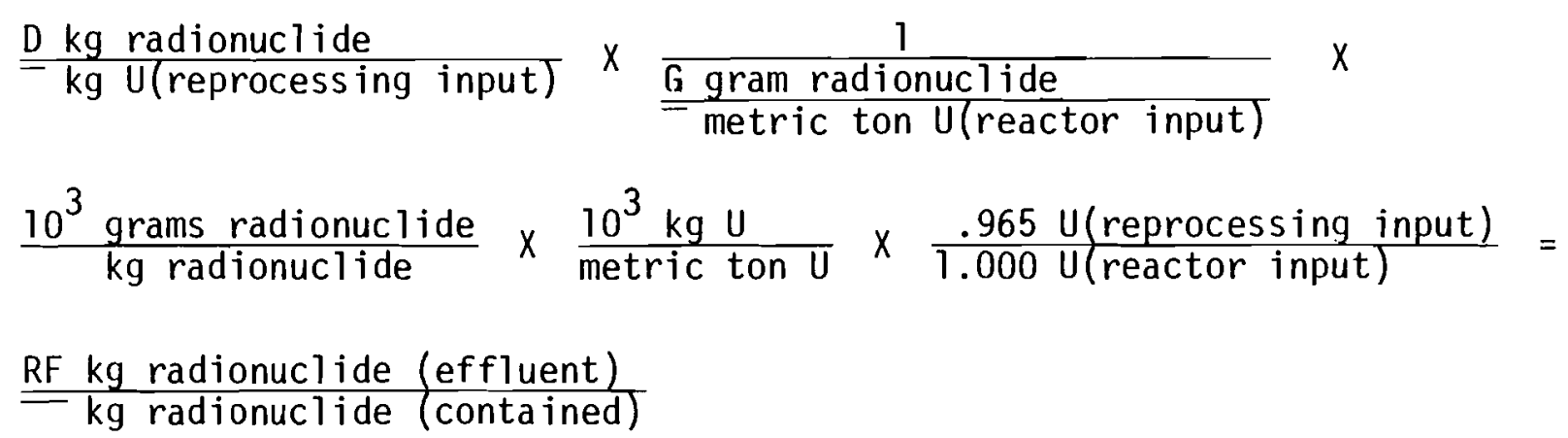

Note: D values come from reference calculations 10-20 through 10-33.

G values come from ORIGEN code, chain 10(180 days cooling time).

RF values are the release factors.

\begin{tabular}{|c|c|c|c|c|}
\hline Isotope & $\mathrm{D}$ & $\mathrm{G}$ & $\mathrm{RF}$ & $\begin{array}{c}\text { Averaged } \\
\text { RF }\end{array}$ \\
\hline 1) Tritium & $3.20 E-8$ & $4.52 E-2$ & $6.83 \mathrm{E}-1$ & $6.83 \mathrm{E}-1$ \\
\hline 2) Krypton-85 & $1.90 \mathrm{E}-5$ & $2.44 E+1$ & $7.51 \mathrm{E}-1$ & 1.0 \\
\hline 3) Strontium-89 & $4.66 E-15$ & 1.93 & $2.33 \mathrm{E}-9$ & \multirow{2}{*}{$2.27 E-9$} \\
\hline 4) Strontium-90 & $1.08 E-12$ & $4.73 E+2$ & $2.20 \mathrm{E}-9$ & \\
\hline 5) Yttrium-90 & $2.80 E-16$ & $1.23 E-1$ & $2.20 \mathrm{E}-9$ & \multirow{2}{*}{$2.50 E-9$} \\
\hline 6) Yttrium-91 & $1.13 \mathrm{E}-14$ & 3.91 & $2.79 \mathrm{E}-9$ & \\
\hline 7) Zirconium-95 & $2.28 \mathrm{E}-14$ & 8.17 & $2.69 E-9$ & $2.69 \mathrm{E}-9$ \\
\hline 8) Niobium-95 & $2.34 \mathrm{E}-14$ & 8.57 & $2.63 E-9$ & $2.63 \mathrm{E}-9$ \\
\hline
\end{tabular}


REFERENCE CALCULATIONS: Reprocessing Facility (cont'd)

\section{Isotope}

9) Ruthenium-103

10) Ruthenium-106

11) Cesium-134

12) Cesium- 137

13) Cerium- 141

14) Cerium-144

15) Promethium- 147

16) Uranium-232

17) Uranium-233

18) Uranium-234

19) Uranium-235

20) Uranium-236

21) Uranium-237

22) Uranium-238

23) Plutonium-238

24) Plutonium-239

25) Plutonium-240

26) Plutonium-241

27) Plutonium-242

28) Americium-241

29) Americium- 242

30) Curium- 242

31) Curium-243

32) Curium-244

33) I odine-129

\begin{tabular}{|c|c|}
\hline D & G \\
\hline $4.54 \mathrm{E}-15$ & 1.34 \\
\hline $2.58 \mathrm{E}-13$ & $9.99 E+1$ \\
\hline $2.54 \mathrm{E}-13$ & $1.28 \mathrm{E}+2$ \\
\hline $2.42 \mathrm{E}-12$ & $1.08 \mathrm{E}+3$ \\
\hline $3.38 \mathrm{E}-15$ & $8.41 \mathrm{E}-1$ \\
\hline $4.56 \mathrm{E}-13$ & $1.89 E+2$ \\
\hline $2.66 \mathrm{E}-13$ & $9.72 \mathrm{E}+1$ \\
\hline $5.58 \mathrm{E}-15$ & $1.98 \mathrm{E}-4$ \\
\hline $3.58 \mathrm{E}-14$ & $2.45 \mathrm{E}-3$ \\
\hline $4.98 \mathrm{E}-11$ & 2.49 \\
\hline $6.12 E-9$ & $7.62 E+3$ \\
\hline $8.40 E-8$ & $3.52 E+3$ \\
\hline $7.50 \mathrm{E}-16$ & $3.40 \mathrm{E}-5$ \\
\hline $1.92 \mathrm{E}-5$ & $9.45 E+5$ \\
\hline $1.73 \mathrm{E}-13$ & $1.23 \mathrm{E}+2$ \\
\hline $4.60 E-12$ & $4.75 E+3$ \\
\hline $2.10 \mathrm{E}-12$ & $2.06 \mathrm{E}+3$ \\
\hline $1.17 \mathrm{E}-12$ & $1.10 E+3$ \\
\hline $5.02 E-13$ & $4.14 E+2$ \\
\hline 1. $72 \mathrm{E}-13$ & $5.71 E+1$ \\
\hline 1. $34 \mathrm{E}-20$ & 1. $35 \mathrm{E}-5$ \\
\hline $1.94 \mathrm{E}-14$ & 5.05 \\
\hline $9.40 E-16$ & $8.71 \mathrm{E}-2$ \\
\hline $8.14 \mathrm{E}-14$ & $1.62 E+1$ \\
\hline $1.59 \mathrm{E}-17$ & $1.70 \mathrm{c}+2$ \\
\hline
\end{tabular}

Averaged

\begin{tabular}{|c|c|}
\hline RF & $\mathrm{RF}$ \\
\hline $3.27 E-9\}$ & \multirow[t]{2}{*}{$2.88 E-$} \\
\hline $2.49 \mathrm{E}-9$ & \\
\hline $1.91 \mathrm{E}-9$ & \multirow[t]{2}{*}{$2.04 \mathrm{E}-9$} \\
\hline $2.16 \mathrm{E}-9$ & \\
\hline $3.88 \mathrm{E}-9$ & \multirow[t]{2}{*}{$3.11 \mathrm{E}-9$} \\
\hline $2.33 E-9$ & \\
\hline $2.64 \mathrm{E}-9$ & \multirow[t]{4}{*}{$2.64 \mathrm{E}-9$} \\
\hline $2.72 \mathrm{E}-5$ & \\
\hline $1.41 \mathrm{E}-5$ & \\
\hline $1.93 \mathrm{E}-5$ & \\
\hline $7.75 \mathrm{E}-7$ & \multirow[t]{6}{*}{$2.08 \mathrm{E}-5$} \\
\hline $2.30 \mathrm{E}-5$ & \\
\hline $2.13 E-5$ & \\
\hline $1.96 \mathrm{E}-5$ & \\
\hline 1. $36 \mathrm{E}-9$ & \\
\hline $9.35 \mathrm{E}-10$ & \\
\hline $9.84 E-10$ & \multirow[t]{3}{*}{$1.10 \mathrm{E}-9$} \\
\hline $1.03 \mathrm{E}-9$ & \\
\hline $1.17 \mathrm{E}-9$ & \\
\hline $2.91 \mathrm{E}-9$ & \multirow[t]{2}{*}{$1.93 \mathrm{E}-9$} \\
\hline $9.58 \mathrm{E}-10$ & \\
\hline $3.71 E-9$ & \multirow{4}{*}{$6.32 \mathrm{E}-9$} \\
\hline $1.04 \mathrm{E}-8$ & \\
\hline $4.85 E-9$ & \\
\hline $8.88 \mathrm{E}-4$ & \\
\hline
\end{tabular}


REFERENCE CALCULATIONS: Reprocessing Facility (cont'd)

5-02-77

REFERENCE 24

Half-life $\quad$ Ci/g

1. Krypton-83M

$1.86 \mathrm{~h}$

2. $03 \mathrm{E}+7$

2. Krypton-85M

$4.39 \mathrm{~h}(4.4)$

8. $40 \mathrm{E}+6$

3. Krypton-85

$10.76 y$

$3.91 \mathrm{E}+2$

4. Krypton-87

$76 \mathrm{~m}$

2. $84 \mathrm{E}+7$

5. Krypton-88

$2.80 \mathrm{~h}$

1. $27 \mathrm{E}+7$

6. Krypton-89

$3.2 \mathrm{~m}$

$6.60 \mathrm{E}+8$

7. Xenon-131M

$11.8 d(12)$

$8.45 \mathrm{E}+4$

8. Xenon-133M

$2.26 \mathrm{~d}(2.3)$

4. $35 E+5$

9. Xenon-133

$5.27 \mathrm{~d}$

$1.86 E+5$

10. Xenon-135M

$15.6 \mathrm{~m}$ (16)

$8.93 E+7$

11. Xenon-135

9. $2 \mathrm{~h}$

$2.52 E+6$

12. Xenon-137

$3.9 m(4.2)$

$3.52 E+8$

13. Xenon-138

$17 \mathrm{~m}$

$8.02 E+7$

14. Iodine-130

12. $3 \mathrm{~h} \mathrm{(12)}$

$1.96 \mathrm{E}+6$

15. Iodine-131

$8.070 \mathrm{~d}(8.05)$

$1.24 E+5$

16. Iodine-132

2. $3 \mathrm{~h}$

$1.03 \mathrm{E}+7$

17. Iodine-133

20.9h (21)

$1.13 \mathrm{E}+6$

18. Iodine-135

$6.7 \mathrm{~h}$

3. $47 \mathrm{E}+6$ 
REFERENCE CALCULATIONS: Reprocessing Facility (cont'd)

REFERENCE 24 (cont'd)

19. Rubidium- 86

20. Rubidium- 88

21. Strontium-89

22. Strontium-90.

23. Strontium-91

24. Yttrium-90

25. Yttrium-91M

26. Yttrium-91

27. Yttrium-93

28. Zirconium-95

29. Zirconium-97

30. Niobium-95

31. Niobium-97M

32. Niobium- 97

33. Molybdenum-99

34. Technitium-99M

Half-life

$\mathrm{Ci} / \mathrm{g}$

$18.66 \mathrm{~d}(18.7)$

$8.14 E+4$

$17.8 \mathrm{~m}(18)$

1. $20 \mathrm{E}+8$

$52 d(50.6)$

$2.82 \mathrm{E}+4$

$28.1 y(28.8)$

$1.42 \mathrm{E}+2$

$9.67 \mathrm{~h}(9.7)$

3. $56 \mathrm{E}+6$

$64 \mathrm{~h}(64.2)$

$5.44 \mathrm{E}+5$

$50 \mathrm{~m}$

4. $13 \mathrm{E}+7$

$58.8 d \quad(59)$

2. $44 E+4$

$10.2 \mathrm{~h}(10.1)$

$3.31 E+6$

$65 d$

$2.12 E+4$

$17 \mathrm{~h}$

$1.90 \mathrm{E}+6$

$35.15 \mathrm{~d} \quad(35)$

3. $91 \mathrm{E}+4$.

$1.0 \mathrm{~m}$

$1.94 \mathrm{E}+9$

$72 m$

2. $69 \mathrm{E}+7$

$66.69 \mathrm{~h}(67)$

4. $75 E+5$

$6.0 \mathrm{~h}$

$5.28 \mathrm{E}+6$

35. Ruthenium-103

$39.6 \mathrm{~d}(40)$

3. $20 \mathrm{E}+4$

36. Ruthenium-106

$367 d$ ( $1 \mathrm{yr}$ )

$3.36 \mathrm{E}+3$

37. Rhodium-103M

(57m)

3. $20 \mathrm{E}+7$

38. Rhodium-105

$35.9 \mathrm{~h} \mathrm{(36)}$

8. $32 E+5$

39. Rhodium-106

$30 \mathrm{~s}$

$3.55 \mathrm{E}+9$ 
REFERENCE CALCULATIONS: Reprocessing Facility (cont'd)

REFERENCE 24 (cont'd)

$\begin{array}{ll}\text { 40. } & \text { Tin-125 } \\ \text { 41. } & \text { Antimony-125 } \\ \text { 42. Antimony-127 } \\ \text { 43. Tellurium-125M } \\ \text { 44. Tellurium-127M } \\ \text { 45. Tellurium-127 } \\ \text { 46. Tellurium-129M } \\ \text { 47. Tellurium-129 } \\ \text { 48. Tellurium-131M } \\ \text { 49. Tellurium-131 } \\ \text { 50. Tellurium-132 } \\ \text { 51. Cesium-134 } \\ \text { 52. Cesium-136 } \\ \text { 53. Cesium-137 } \\ \text { 54. Barium-137M } \\ \text { 55. Barium-140 } \\ \text { 56. Lanthanum-140 } \\ \text { 57. Cerium-141 } \\ \text { 58. Cerium-143 } \\ 59 . \quad \text { Cerium-144 } \\ 60 . & \text { Praseodymium-143 }\end{array}$

\begin{tabular}{|c|c|}
\hline $\mathrm{Half}-1$ ife & $\mathrm{Ci} / \mathrm{g}$ \\
\hline $9.4 d(9.62)$ & $1.11 E+5$ \\
\hline $2.7 y$ & $1.06 \mathrm{E}+3$ \\
\hline $93 h(3.9 d)$ & $2.65 E+5$ \\
\hline $58 d$ & $1.80 \mathrm{E}+4$ \\
\hline $109 d(105)$ & $9.44 E+3$ \\
\hline $9.4 \mathrm{~h}(9.3)$ & $2.63 E+6$ \\
\hline $34 d$ & $2.98 E+4$ \\
\hline $69 m(67)$ & $2.11 E+7$ \\
\hline $30 \mathrm{~h}(1.2 \mathrm{~d})$ & $7.98 \mathrm{E}+5$ \\
\hline $25 m$ & $5.74 \mathrm{E}+7$ \\
\hline $78 \mathrm{~h}$ & $3.05 E+5$ \\
\hline $2.05 y \quad(2.1)$ & $1.30 E+3$ \\
\hline $13 d$ & $7.39 E+4$ \\
\hline $30.23 y \quad(30)$ & $8.64 E+1$ \\
\hline $2.55 \mathrm{~m}(2.6)$ & $5.38 E+8$ \\
\hline $12.8 d$ & $7.29 E+4$ \\
\hline $40.22 \mathrm{~h}(40.2)$ & $5.57 E+5$ \\
\hline $33 d(32.5)$ & $2.81 E+4$ \\
\hline $33 h$ & $6.64 \mathrm{E}+5$ \\
\hline $284.9 d \quad(285)$ & $3.18 E+3$ \\
\hline $13.7 \mathrm{~d}$ & $6.67 E+4$ \\
\hline
\end{tabular}


REFERENCE CALCULATIONS: Reprocessing Facility (cont'd)

REFERENCE 24 (cont'd)

61. Praseodymium-144

\begin{tabular}{ll} 
Half-life & \multicolumn{1}{c}{$\mathrm{Ci} / \mathrm{g}$} \\
\hline $17.3 \mathrm{~m}$ & $7.55 \mathrm{E}+7$ \\
$11.1 \mathrm{~d}$ & $8.01 \mathrm{E}+4$ \\
$2.5 \mathrm{y}(2.6)$ & $9.74 \mathrm{E}+2$ \\
$27.8 \mathrm{~d}$ & $9.21 \mathrm{E}+4$ \\
$303 \mathrm{~d}(312)$ & $7.98 \mathrm{E}+3$ \\
$2.6 \mathrm{y}(2.4)$ & $2.50 \mathrm{E}+3$ \\
$45.1 \mathrm{~d}(45)$ & $4.91 \mathrm{E}+4$ \\
$71.3 \mathrm{~d}(71)$ & $3.16 \mathrm{E}+4$ \\
$5.26 \mathrm{y}(5.24)$ & $1.13 \mathrm{E}+3$ \\
$12.26 \mathrm{y}$ & $9.73 \mathrm{E}+3$ \\
$1.83 \mathrm{~h}$ & $4.18 \mathrm{E}+7$ \\
$243.6 \mathrm{~d}$ & $8.25 \mathrm{E}+3$ \\
$60.3 \mathrm{~d}$ & $1.75 \mathrm{E}+4$ \\
$5730 \mathrm{y}$ & 4.46 \\
$15.0 \mathrm{~h}$ & $8.71 \mathrm{E}+6$ \\
$14.3 \mathrm{~d}$ & $2.85 \mathrm{E}+5$ \\
$2.576 \mathrm{~h}$ & $2.17 \mathrm{E}+7$ \\
$2.521 \mathrm{~h}$ & $1.91 \mathrm{E}+7$ \\
$12.9 \mathrm{~h}$ & $3.80 \mathrm{E}+6$ \\
$13.9 \mathrm{~h}$ & $3.27 \mathrm{E}+6$ \\
& \\
\hline 10 &
\end{tabular}

62. Neodymium-147

63. Promethium-147

64. Chromium-51

65. Manganese-54

66. Iron-55

67. Iron-59

68. Cobalt-58

69. Cobalt-60

70. Tritium

71. Argon-41

72. Zinc -65

73. Antimony-124

74. Carbon-14

75. Sodium-24

76. Phosphorus -32

77. Manganese-56

78. Nickel-65

79. Copper-64

80. Zinc-69M

$7.55 \mathrm{E}+7$

9. $74 \mathrm{E}+2$

$9.21 \mathrm{E}+4$

$7.98 E+3$

$50 \mathrm{E}+3$

$91 E+4$

$.13 E+3$

9.73E+3

4. $18 \mathrm{E}+7$

4.46

$8.71 \mathrm{E}+6$

2. $85 E+5$

$3.27 \mathrm{E}+6$ 
REFERENCE CALCULATIONS: Reprocessing Facility (cont'd)

REFERENCE 24 (cont'd)

81. Zinc-69

82. Wol fram-187

83. Neptunium-239

84. Bromine-83

85. Bromine- 84

86. Rubidium-89

87. Strontium-92

88. Yttrium-92

89. Niobium-98

90. Technitium-101

91. Technitium-104

92. Ruthenium-105

93. Rhodium-105M

94. Iodine-134

95. Cesium-138

96. Barium-139

97. Lanthanum-141

98. Lanthanum-142
Half-life

$58 \mathrm{~m}$

$24 \mathrm{~h}$

2. $35 d$

$2.41 \mathrm{~h}$

$31.8 \mathrm{~m}$

$15.4 \mathrm{~m}$

$2.71 \mathrm{~h}$

$3.53 \mathrm{~h}$

$51 \mathrm{~m}$

$14 m$

$18 \mathrm{~m}$

$4.44 \mathrm{~h}$

$45 \mathrm{~s}$

$52 \mathrm{~m}$

$32.2 \mathrm{~m}$

$82.9 \mathrm{~m}$

$3.9 \mathrm{~h}$

$92 m$
$\mathrm{Ci} / \mathrm{g}$

4. $70 \mathrm{E}+7$

6. $99 \mathrm{E}+5$

2. $33 E+5$

1. $57 \mathrm{E}+7$

$7.04 \mathrm{E}+7$

1. $37 \mathrm{E}+8$

1. $26 \mathrm{E}+7$

9. $65 \mathrm{E}+6$

3. $76 \mathrm{E}+7$

1. $33 E+8$

1. $00 E+8$

$6.72 E+6$

2. $39 E+9$

2. $70 E+7$

$4.23 E+7$

1. $63 \mathrm{E}+7$

$5.70 E+6$

1. $44 \mathrm{E}+7$

( ) Chart of Nuclides (1969) 
REFERENCE CALCULATIONS: Reprocessing Facility (cont'd)

REFERENCE 30

Half-life

1. Hydrogen-3

2. Carbon-14

3. Krypton-85

4. Strontium-89

5. Strontium-90

6. Yttrium-90

7. Yttrium-91

8. Zirconium-95

9. Niobium-95

10. Ruthen ium-103

11. Ruthenium-106

12. Silver-110M

13. Antimony-125

14. Tellurium-127M

15. Tellurium-129M

16. Iodine-129

17. Iodine-131

18. Cesium-134

19. Cesium-137
$12.26 y$

$5730 y$

$10.76 y$

$52 d$

$28.1 y$

$64 \mathrm{~h}$

$58.8 d$

$65 d$

$35.15 \mathrm{~d}$

$39.6 d$

$367 d$

253d

$2.7 y$

$109 \mathrm{~d}$

$34 d$

$1.7 \times 10 \mathrm{E}+7 \mathrm{y}$

$8.070 \mathrm{~d}$

$2.05 y$

$30.23 y$
$\mathrm{Ci} / \mathrm{g}$

$9.73 \mathrm{E}+3$

4.46

$3.91 \mathrm{E}+2$

2. $82 \mathrm{E}+4$

1. $42 \mathrm{E}+2$

5. $44 \mathrm{E}+5$

2. $44 \mathrm{E}+4$

2. $12 E+4$

$3.91 E+4$

3. $20 \mathrm{E}+4$

3. $36 \mathrm{E}+3$

$4.69 \mathrm{E}+3$

1. $06 \mathrm{E}+3$

9. $44 \mathrm{E}+3$

$2.98 \mathrm{E}+4$

1. $63 \mathrm{E}-4$

1. $24 \mathrm{E}+5$

1. $30 \mathrm{E}+3$

8. $64 \mathrm{E}+1$ 
REFERENCE CALCULATIONS: Reprocessing Facility (cont'd)

REFERENCE 30 (cont'd)

20. Cerium-141

Half-life

$\mathrm{Ci} / \mathrm{g}$

21. Cerium-144

$33 d$

2. $81 \mathrm{E}+4$

22. Promethium-147

$284.9 d$

$3.18 \mathrm{E}+3$

23. Europium-154

$2.5 y$

9. $74 \mathrm{E}+2$

24. Europium-155

$16 y$

$1.45 \mathrm{E}+2$

25. Uranium-232

$1.81 y$

1. $28 \mathrm{E}+3$

$73.6 \mathrm{y}$

2. $10 \mathrm{E}+1$

26. Uranium-233

$1.62 \times 10 \mathrm{E}+5 \mathrm{y}$

9. $48 \mathrm{E}-3$

27. Uranium-234

$2.47 \times 10 \mathrm{E}+5 \mathrm{y}$

$6.19 \mathrm{E}-3$

28. Uranium-235

29. Uranium-236

$7.1 \times 10 \mathrm{E}+8 \mathrm{y}$

2. $14 \mathrm{E}-6$

$2.39 \times 10+7 y$

$6.35 \mathrm{E}-5$

30. Uranium-237

$6.75 d$

$8.17 E+4$

31. Uranium-238

$4.51 \times 10 E+9 y$

3. $33 \mathrm{E}-7$

32. Plutonium-238

$86 y$

1. $75 \mathrm{E}+1$

33. Plutonium-239

34. Plutonium-240

$2.44 \times 10 E+4 y$

$6.14 E-2$

$6580 y$

2. $27 \mathrm{E}-1$

$13.2 y$

1. $13 \mathrm{E}+2$

35. Plutonium-241

$3.79 \times 10 E+5 y$

3. $90 \mathrm{E}-3$

37. Americium-241

$458 y$

3.24

16. $0 \mathrm{~h}$

8. $10 E+5$

39. Americium-243

$7.37 \times 10 \mathrm{E}+3 \mathrm{y}$

2.00E-1

40. Curium-242

$163 d$

$3.31 E+3$

41. Curium-243

$32 y$

$4.60 \mathrm{E}+1$

42. Curium-244

$17.6 y$

8. $33 \mathrm{E}+1$ 


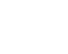




\section{APPENDIX A: REFERENCES}

1. Compilation of Air Pollutant Emission Factors, U.S. EPA, AP-42, Apri1 1973.

2. Development Document for Interim Final and Proposed Effluent Limitations Guidelines and New Source Performance Standards for the Ore Mining and Dressing Industry. Point Source Category Vol. I \& II. October 1975. EPA 440/1-75/061.

3. Water Quality Impacts of Uranium Mining and Milling Activities in the Grants Mineral Belt, New Mexico, U.S. EPA, EPA 906/9-75-002, September 1975.

4. Handbook of Chemistry and Physics, by The Chemical Rubber Company, editor Robert C. Weast, Ph.D., 1970-1971 edition.

5. Toxic Effluents from Uranium Mining, by R. L. McKeever and W. A. Muckerheide, Battelle Pacific Northwest Laboratories, March 1977 (see Appendix C).

6. Final Environmental Statement related to operation of the Highland Uranium Mi11, submitted by U.S. AEC, Docket 408102-7, March 1973.

7. Applicant's Environmental Report, Highland Uranium Mil1, Converse County, Wyoming submitted by Humble $0 i 1$ and Refining Company, Docket 408102-1, July 1971.

8. M. B. Sears, et a1., Correlation of Radioactive Waste Treatment Costs and the Environmental Iinpact of Waste Effluents in the Nuclear Fuel Cycle for Use in Establishing "As Low As Possible" Guides - Milling of Uranium Ores, ORNL-TM-4903, Vo1. I., May 1975.

9. W. J. Shelley, Kerr-McGee Nuclear Corporation, Oklahoma City, Oklahoma, letter and attachment to Richard B. Chitwood, Nuclear Regulatory Commission, Public Document Room, Washington, D.C., dated June 27, 1975.

10. Applicant's Environmental Report, Supplemental (Supplement $\# 1$ ), Sequoyah Uranium Hexafluoride Plant, Kerr-McGee Corporation, Docket 40-8027-9, June 1972.

11. Final Environmental Statement related to the Sequoyah Uranium Hexaf7uoride Plant, U.S. NRC, Docket 40-8027 or NUREG-75/007, February 1975.

12. W. J. Shelley, Kerr-McGee Nuclear Corporation, Oklahoma City, Oklahoma, letter and attachment to Richard B. Chitwood, Nuclear Regulatory Commission, Public Document Room, Washington, D.C., dated June 5, 1975.

13. AEC Gaseous Diffusion Plant Operations, U.S. AEC, ORO-684, January 1972. 
14. Final Environmental Statement, Expansion of U.S. Uranium Enrichment Capacity, ERDA, ERDA-1543, Apri1 1976.

15. Draft Environmental Statement, Portsmouth Gaseous Diffusion Plant Expansion, ERDA, ERDA-1549, Volumes 1 and 2, June 1976.

16. Environmental Effects of the Construction and Operation of a Gaseous Diffusion Plant, U.S. AEC, ORO-725, July 26, 1973.

17. Environmental Survey of the Uranium Fuel Cycle, U.S.A.E.C., WASH-1248, April 1974.

18. Westinghouse Nuclear Fuel Columbia Site, Evaluation Report, Westinghouse Nuclear Fuel Division, Docket 70-1151, March 1, 1975.

19. Final Environmental Statement - Portsmouth Gaseous Diffusion Plant Expansion, Piketon, Ohio, ERDA-1549, September 1977.

20. Nomographs, pages 40 and 41 from Reference 13 to provide enrichment from $0.711 \%$ to $3.2 \%$ with $0.25 \%$ tails.

21. Recycle Fuels Plant, Environmental Report, Westinghouse Nuclear Fuel Division, July 1973 (no document number assigned).

22. From Section $11 \mathrm{~A}$ of this report.

23. Final Environmental Statement related to the operation of Trojan Nuclear Plant, U.S. AEC, Docket No. 50-344, August 1973.

24. Appendix A, reference sheet of specific activities (curies per gram) and half-lives of nuclides based on reference 4 information.

25. \& 26. Final Safety Analys is Report, WNP-2, Washington Public Power Supply System, U.S. NRC Docket No. 50-397.

27. Final Environmental Statement related to the constructiona and operation of Barnwel1 Nuclear Fuel Plant, U.S. AEC, Docket No. 50-332, January 1974.

28. Revision No. 3, Environmental Report, UF 6 Facility, Barnwell Nuclear Fuel Plant, A1lied-General Nuclear Services, Docket No. 70-1327, September 1974 .

29. Oraft Supplement to the Final Environmental Statement related to the construction and operation of Barnwe11 Nuclear Fuel Plant, U.S. Nuclear Regulatory Commission, Docket No. 50-332, NUREG-0082, Supp. 1 (Draft), June 1976. 
30. Appendix A reference sheet of specific activity calculations based on Reference 4 information.

31. Preliminary Safety Analys is Report, Addendum No. 7, Plutonium Product Facility, AEC Docket No. 70-1193, September 29, 1971.

32. Final Safety Analys is Report, Amendment 29, Trojan, Portland General Electric Company, U.S. NRC Docket No. 50-344-(282) June 2, 1976.

33. Final Safety Analysis Report, WNP-2, Washington Public Power Supply System, NRC Docket No. 397.

34. G. H. Clark, Assessment of the Meteorological Data and Atmospheric Dispersion Estimates in the Ranger I Uranium Mining Environmenta 7 Impact Statement, AAEC/E407, Australian Atomic Energy Commission, March, 1977.

35. K. K. Nielson, R. W. Perkins, L. L. Schwendiman, W. I. Enerlin, Prediction of the Net Radon Emission from a Model Open Pit Uranium Mine, NUREG/CR-0628, PNL-2889, Pacific Northwest Laboratory, RichTand, WA 99352, Apri1 1979.

36. P. 0. Jackson, et al., Radon-222 Emissions in Ventilation Air Exhausted from Underground Uranium Mines, NUREG/CR-0627, PNL 2888, Pacific Northwest Laboratory, Richland, WA 99352, March 1979.

37. W. F. Marshal1, D. E. Seizinger, and R. W. Freedman, Effects of Catalytic Reactors on Diesel Exhaust Composition, Health and Safety Research - Coal Mines Program, Technical Progress Report 105, U.S. Bureau of the Interior, April 1978.

38. Licensee's Environmental Report Renewal of Source Material License SUA-1139, Exxon Minerals Company, U.S.A., Docket No. 40-8102, Highl and Uranium Mi11, Converse County, Wyoming, June 1978.

39. James D. Patton, Exxon Minerals Company, U.S.A., Houston, Texas, letter and attachment to R. L. Auberg, Pacific Northwest Laboratory, Richland, WA 99352. Dated May 29, 1979. 


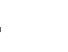




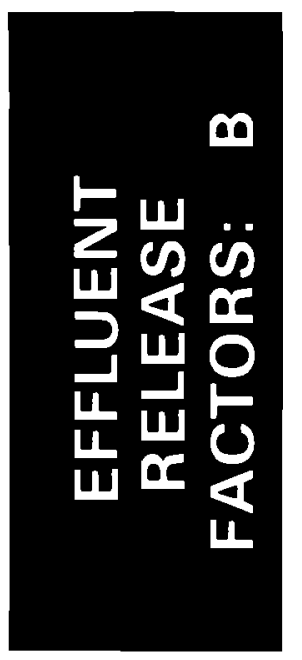




\title{
ADDITIONAL DERIVATIONS OF EFFLUENT RELEASE FACTORS FOR THE REFERENCE FUEL CYCLE FACILITIES
}

\author{
M. H. Arndt, J. K. Young
}

Appendix $B$ contains the derivations of radioactive carbon-14 release factors for the reference boiling water reactor, pressurized water reactor and reprocessing facilities.

A release factor is defined in units of kilograms released per kilogram of heavy metal processed for each chemical species. The release factors are assigned a reliability rating of 1,2 , or 3 based on the following assumptions:

1. A reasonable but unsubstantiated guess

2. Calculated or based on laboratory measurement

3. Based on some plant experience

The reference plants studied were:

\section{Facility}

Surface Mine
Underground Mine
Acid Leach Mil1
Aqueous Conversion
Gaseous Diffusion Enrichment
Uranium Fuel Fabrication
Pressurized Water Reactor
Boiling Water Reactor
Fuel Reprocessing

Page Number of Summary Table

$\mathrm{B}-2$

$B-8$

$B-17$

$B-26$

$B-34$

$B-30$

$B-39$

$B-47$

B-53 
The summary tables present the release factor, the reliability rating and the number of the reference calculation for individual chemicals released from the facility. These release factors supplement those found in Appendix A.

$$
\text { TABLE B-1. } \begin{aligned}
& \text { Surface Mine - Chemical P.elease Factors } \\
& \mathrm{kg} / \mathrm{kgU} \text { (input) }
\end{aligned}
$$

\begin{tabular}{|c|c|c|c|c|c|c|}
\hline \multirow[b]{2}{*}{ Haterial Released } & \multicolumn{3}{|c|}{ Airborne } & \multicolumn{3}{|c|}{ Liguid } \\
\hline & Factor & Reliability & $\begin{array}{l}\text { Ref. } \\
\text { Calc. }\end{array}$ & Factor & Reliability & $\begin{array}{l}\text { Ref. } \\
\text { Calc. } \\
\end{array}$ \\
\hline \multicolumn{7}{|l|}{ Thorium-230 } \\
\hline \multicolumn{7}{|l|}{ Hydrogen Sulfide } \\
\hline \multicolumn{7}{|l|}{ Chloride } \\
\hline \multicolumn{7}{|l|}{ Sulfate } \\
\hline Arsenic & & & & $4 \times 10^{-5}$ & 1 & B. 1.2 .1 \\
\hline Selenium & & & & $2 \times 10^{-5}$ & 1 & B. 1.2 .2 \\
\hline Armonia & & & & $3 \times 10^{-4}$ & 1 & B.1.2.3 \\
\hline Kjeldahl Nitrogen & & & & $8 \times 10^{-4}$ & 1 & B.1.2.4 \\
\hline Witrate & & & & $1 \times 10^{-4}$ & 1 & B.2.2.5 \\
\hline Phosphorus & & & & $6 \times 10^{-4}$ & 1 & B.1.2.6 \\
\hline Fluoride & & & & $4 \times 10^{-4}$ & 1 & B.1.2.7 \\
\hline Aluminum & & & & $4 \times 10^{-4}$ & 1 & B.1.2.8 \\
\hline Beryllium & & & & $8 \times 10^{-6}$ & 1 & B. 1.2 .9 \\
\hline Calcium & & & & $8 \times 10^{-2}$ & 1 & B.1.2.10 \\
\hline Magnesium & & & & $3 \times 10^{-2}$ & 1 & B.1.2.11 \\
\hline Titanium & & & & $7 \times 10^{-4}$ & 1 & B.1.2.12 \\
\hline Molybdenum & & & & $7 \times 10^{-4}$ & 1 & B.1.2.13 \\
\hline Vanadium & & & & $1 \times 10^{-3}$ & 1 & B.1.2.14 \\
\hline Iron & & & & $6 \times 10^{-3}$ & 1 & B.1.2.15 \\
\hline Lead & & & & $1 \times 10^{-4}$ & 1 & B.1.2.16 \\
\hline Zinc & & & & $2 \times 10^{-5}$ & 1 & B.1.2.17 \\
\hline
\end{tabular}




\section{B. 1 SURFACE MINE CALCULATIONS}

\section{B.1.1 ASSUMPTIONS}

The largest liquid waste stream from open pit mining operations is the mine drainage water. The mine water discharge for a similar model mine with a capacity of $960 \mathrm{MT} \mathrm{U}_{3} \mathrm{O}_{8} / \mathrm{yr}$ is about $1500 \mathrm{gpm}$ (Reference 4, pp. A-9). The capacity of the ENFORM model mine is $2337 \mathrm{MT} \mathrm{U}_{3} \mathrm{O}_{8} / \mathrm{yr}$. therefore the following scale-up is utilized to assume a reasonable mine-water discharge rate for calculations.

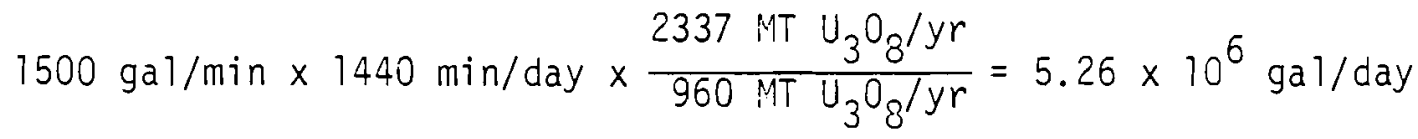

The mine drainage water is pumped from the mine to keep the mine dry while ore is being extracted. Only part of this water becomes surface run-off (arbitrarily assumed 25\%) while the bulk recycles through natural seepage and evaporation (Reference 4, pp. A-9).

$$
5.26 \times 10^{6} \mathrm{gal} / \text { day } \times .25=1.31 \times 10^{6} \mathrm{gal} / \text { day }
$$

Therefore, only $1.31 \times 10^{5} \mathrm{gpd}$ of the mine drainage stream is assumed an effluent.

Reference 5 data will be used for surface mine calculations (see page B-9 for explanation).

\section{B.1.2 LIQUID RELEASES}

B.1.2.1 Arsenic

(4, pp. A-10)

$.05 \mathrm{mg} / \ell \times \frac{\mathrm{kg}}{10^{6} \mathrm{mg}} \times 3.785 \mathrm{\ell} / \mathrm{gal} \times 1.31 \times 10^{6} \mathrm{gal} /$ day $=.25 \mathrm{~kg} \mathrm{As} /$ day 


$$
\therefore \frac{.25 \mathrm{~kg} \mathrm{As} / \mathrm{day}}{6350 \mathrm{~kg} \mathrm{~J} / \mathrm{day}}=4 \times 10^{-5} \frac{\mathrm{kg}}{\mathrm{kg} \mathrm{J}(\text { Anput })}
$$

\section{B.1.2.2 Seleniuir}

$$
\begin{aligned}
& (4, \mathrm{p} \cdot \mathrm{A}-10) \\
& .02 \mathrm{mg} / 2 \times \frac{\mathrm{kg}}{10^{6} \mathrm{mg}} \times 3.785 \mathrm{R} / \mathrm{ga} 1 \times 1.31 \times 10^{6} \mathrm{ga} 1 / \mathrm{day}=.10 \mathrm{~kg} \mathrm{Se} / \mathrm{day} \\
& \therefore \frac{.10 \mathrm{~kg} \mathrm{Se} / \mathrm{day}}{6350 \mathrm{~kg} \mathrm{U} / \mathrm{day}}=1.6 \times 10^{-5} \frac{\mathrm{kg} \mathrm{Se}}{\mathrm{kgU} \text { (input) }}
\end{aligned}
$$

B.1.2.3 Ammonia

$$
\begin{aligned}
& (4, \mathrm{P} \cdot \mathrm{A}-12) \\
& \frac{.22+1.33+1.51}{3} \mathrm{mg} / 2 \times \frac{\mathrm{kg}}{10^{6} \mathrm{mg}} \times 3.735 \mathrm{\ell} / \mathrm{gal} \times 1.31 \times 10^{6} \mathrm{ga} 1 / \text { day } \\
& =5.08 \mathrm{~kg} \mathrm{NH} / \text { day } \\
& \therefore \frac{5.08 \mathrm{~kg} \mathrm{iNH} / \text { day }}{6350 \mathrm{~kg} \mathrm{U} / \text { day }}=8.0 \times 10^{-4} \frac{\mathrm{kg} \mathrm{iNH_{3 }}}{\mathrm{kg} \mathrm{U}}
\end{aligned}
$$

\section{B.1.2.4 Kjeldahl Nitrogen}

$$
\begin{aligned}
& (4, \mathrm{p} \cdot \mathrm{A}-12) \\
& \frac{.22+1.33+1.42}{3} \mathrm{mg} / \ell \times \frac{\mathrm{kg}}{10^{6} \mathrm{mg}} \times 3.785 \mathrm{\ell} / \mathrm{gal} \times 1.31 \times 10^{6} \mathrm{gal} / \mathrm{day} \\
& =4.9 \mathrm{~kg} \mathrm{~N} / \mathrm{day} \\
& \therefore \frac{4.9 \mathrm{~kg} / \mathrm{day}}{6350 \mathrm{~kg} \mathrm{U} / \mathrm{day}}=7.8 \times 10^{-4} \frac{\mathrm{kg} \mathrm{i}}{\mathrm{kg} \mathrm{U}}
\end{aligned}
$$

B.1.2.5 Nitrate

$$
\begin{aligned}
& (4, \mathrm{p} \cdot \mathrm{A}-12) \\
& \frac{.01+.002+.53}{3} \mathrm{mg} / \ell \times \frac{\mathrm{kg}}{10^{6} \mathrm{mg}} \times 3.785 \mathrm{\ell} / \mathrm{gal} \times 1.31 \times 10^{6} \mathrm{gal} / \mathrm{day} \\
& =0.90 \mathrm{~kg} \mathrm{NO} / \mathrm{day} \\
& \therefore \frac{6350 \mathrm{~kg} \mathrm{U} / \text { day }}{6}=1.4 \times 10^{-4} \frac{\mathrm{kg} \mathrm{NO}}{\mathrm{kg} \mathrm{U^{3 }}}
\end{aligned}
$$




\section{B.1.2.6 Fhosphorus}

$$
\begin{aligned}
& (4, \mathrm{p} \cdot \mathrm{A}-12) \\
& \left(\frac{.05+.07+2.3}{3}\right) \mathrm{mg} / \mathrm{i} \times \frac{\mathrm{kg}}{10^{6} \mathrm{mg}} \times 3.785 \AA / \mathrm{gal} \times 1.31 \times 10^{6} \mathrm{ga} 1 / \text { day } \\
& =4.0 \mathrm{~kg} \mathrm{PO} / \mathrm{day} \\
& \therefore \frac{4.0 \mathrm{~kg} \mathrm{PO} / \mathrm{day}}{6350 \mathrm{~kg} \mathrm{U} / \text { day }}=6.3 \times 10^{-4} \frac{\mathrm{kg} \mathrm{PO}_{4}}{\mathrm{~kg} \mathrm{U}}
\end{aligned}
$$

\section{B.1.2.7 Fluoride}

(5, p. 336)

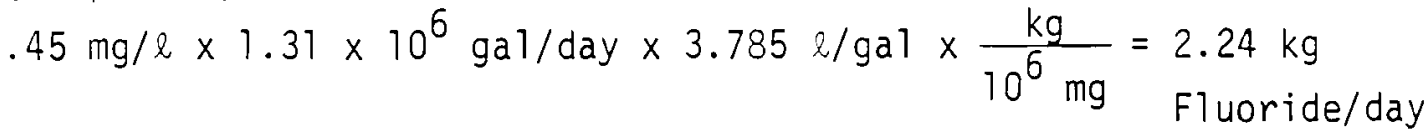

$\therefore \frac{2.24 \mathrm{~kg} \mathrm{Fluoride} / \text { day }}{6350 \mathrm{~kg} \mathrm{U} / \mathrm{day}}=3.5 \times 10^{-4} \frac{\mathrm{kg} \mathrm{Fluoride}}{\mathrm{kg} \mathrm{U}}$

\section{B.1.2.8 Aluminum}

(Same as Fluoride)

\section{B.1.2.9 Beryllium}

(5, p. 336)

$.01 \mathrm{mg} / \mathrm{\ell} \times 1.31 \times 10^{6} \mathrm{gal} /$ day $\times 3.785 \mathrm{l} / \mathrm{ga} 1 \times \frac{\mathrm{kg}}{10^{6 \mathrm{mg}}}=.05 \mathrm{~kg} \mathrm{Be} /$ day

$\therefore \frac{.05 \mathrm{~kg} \mathrm{Be} / \text { day }}{6350 \mathrm{~kg} \mathrm{U} / \text { day }}=7.81 \times 10^{-6} \frac{\mathrm{kg} \mathrm{Be}}{\mathrm{kg} \mathrm{U}}$

\section{B.1.2.10 Calcium}

$$
(5, \text { p. 336) }
$$

$\left(\frac{90+120}{2}\right) \mathrm{mg} / \ell \times 1.31 \times 10^{6} \mathrm{gal} /$ day $\times 3.785 \mathrm{\ell} / \mathrm{gal} \times \frac{\mathrm{kg}}{10^{6 \mathrm{mg}}}=522 \mathrm{~kg} \mathrm{Ca} / \mathrm{day}$

$\therefore \frac{522 \mathrm{~kg} \mathrm{Ca} / \mathrm{day}}{6350 \mathrm{~kg} \mathrm{U} / \mathrm{day}}=.082 \frac{\mathrm{kg} \mathrm{Ca}}{\mathrm{kg} \mathrm{U}}$ 


\section{B.1.2.11 ilagnesium}

$(5$, p. 336)

$40 \mathrm{mg} / \mathrm{i} \times 1.31 \times 10^{6} \mathrm{gal} / \mathrm{day} \times 3.785 \therefore / \mathrm{ga} 1 \times \frac{\mathrm{kg}}{10^{5} \mathrm{mg}}=199.0 \mathrm{~kg} \mathrm{ig} / \mathrm{day}$ $\therefore \frac{199.0 \mathrm{~kg} \mathrm{Mg} / \text { day }}{6350 \mathrm{~kg} \mathrm{U} / \mathrm{day}}=.031 \frac{\mathrm{kg} \mathrm{i.1g}}{\mathrm{kg} \mathrm{U}}$

B.1.2.12 Titanium

(5, p. 336)

$.95 \mathrm{mg} / 2 \times 1.31 \times 10^{6} \mathrm{ga} 1 /$ day $\times 3.785 \mathrm{\ell} / \mathrm{ga} 1 \times \frac{\mathrm{kg}}{10^{6} \mathrm{mg}}=4.7 \mathrm{~kg} \mathrm{Ti} / \mathrm{day}$

$\therefore \frac{4.7 \mathrm{~kg} \mathrm{Ti} / \text { day }}{6350 \mathrm{~kg} \mathrm{U} / \mathrm{day}}=7.4 \times 10^{-4} \frac{\mathrm{kg} \mathrm{Ti}}{\mathrm{kg} \mathrm{U}}$

B.1.2.13 Molybdenum

(5, p. 336)

$.85 \mathrm{mg} / \ell \times 1.31 \times 10^{6} \mathrm{gal} /$ day $\times 3.785 \mathrm{\ell} / \mathrm{ga} 7 \times \frac{\mathrm{kg}}{10^{6} \mathrm{mg}}=4.2 \mathrm{~kg} \mathrm{Mo} / \mathrm{day}$

$\therefore \frac{4.2 \mathrm{~kg} \mathrm{Mo} / \text { day }}{6350 \mathrm{~kg} \mathrm{U} / \mathrm{day}}=6.7 \times 10^{-4} \frac{\mathrm{kg} \mathrm{Mo}}{\mathrm{kg} \mathrm{U}}$

B.1.2.14 Vanadium

$(5, \text { p. } 336)^{(a)}$

$1.3 \mathrm{mg} / \ell \times 1.31 \times 10^{6} \mathrm{gal} /$ day $\times 3.7852 / \mathrm{gal} \times \frac{\mathrm{kg}}{10^{6} \mathrm{mg}}=6.5 \mathrm{~kg} \mathrm{~V} /$ day

$\therefore \frac{6.5 \mathrm{~kg} \mathrm{~V} / \text { day }}{6350 \mathrm{~kg} \mathrm{U} / \text { day }}=1 \times 10^{-3} \frac{\mathrm{kg} \mathrm{V}}{\mathrm{kg} \mathrm{U}}$

(a) 0.5 to $2.1 \mathrm{mg} / \mathrm{l}$; assumed average of 1.3 
B.1.2.15 Iron

(5, p. 337)

$7.6 \mathrm{mg} / 2 \times 1.31 \times 10^{6} \mathrm{gal} /$ day $\times 3.7852 / \mathrm{gal} \times \frac{\mathrm{kg}}{10^{6} \mathrm{mg}}=37.8 \mathrm{~kg} \mathrm{Fe} /$ day

$\therefore \frac{37.8 \mathrm{~kg} \mathrm{Fe} / \text { day }}{6350 \mathrm{~kg} \mathrm{U} / \text { day }}=6 \times 10^{-3} \frac{\mathrm{kg} \mathrm{Fe}}{\mathrm{kg} \mathrm{U}}$

B. 1.2.16 Lead

(5, p. 337)

$.14 \mathrm{mg} / \ell \times 1.31 \times 10^{6} \mathrm{gal} /$ day $\times 3.785 \ell / \mathrm{ga} 1 \times \frac{\mathrm{kg}}{10^{6} \mathrm{mg}}=0.70 \mathrm{~kg} \mathrm{~Pb} / \mathrm{day}$

$\therefore \frac{0.70 \mathrm{~kg} \mathrm{~Pb} / \text { day }}{6350 \mathrm{~kg} \mathrm{U} / \mathrm{day}}=1.10 \times 10^{-4} \frac{\mathrm{kg} \mathrm{Pb}}{\mathrm{kg} \mathrm{U}}$

B.1.2.17 Zinc

(5, p. 337)

$.025 \mathrm{mg} / \ell \times 1.31 \times 10^{6} \mathrm{ga} 1 /$ day $\times 3.785 \mathrm{\ell} / \mathrm{ga} 1 \times \frac{\mathrm{kg}}{10^{6} \mathrm{mg}}=0.12 \mathrm{~kg} \mathrm{Zn} /$ day

$\therefore \frac{0.12 \mathrm{~kg} \mathrm{Zn} / \text { day }}{6350 \mathrm{~kg} \mathrm{U} / \mathrm{day}}=1.96 \times 10^{-5} \frac{\mathrm{kg} \mathrm{Zn}}{\mathrm{kg} \mathrm{U}}$ 
TABLE B-2. Underground Mine - Chemical Release Factors $\mathrm{kg} / \mathrm{kgU}$ (inout)

\begin{tabular}{|c|c|c|c|c|c|c|}
\hline \multirow[b]{2}{*}{ Material Released } & \multicolumn{3}{|c|}{ Airborne } & \multicolumn{3}{|c|}{ Liquid } \\
\hline & Factor & Reliabijity & $\begin{array}{l}\text { Ref. } \\
\text { Calc. }\end{array}$ & Factor & Reliability & $\begin{array}{c}\text { Ref. } \\
\text { Calc. } \\
\end{array}$ \\
\hline \multicolumn{7}{|l|}{ Hydrogen Sulfide } \\
\hline \multicolumn{7}{|l|}{ Thor ium-230 } \\
\hline Benzo(a)pyrene & $7 \times 10^{-8}$ & 2 & 3.2.2.1 & & & \\
\hline Beryllium & $1 \times 10^{-6}$ & 2 & B.2.2.2 & $1 \times 10^{-5}$ & $?$ & B.2.3.16 \\
\hline Cadmium & $2 \times 10^{-6}$ & 2 & B.2.2.3 & & & \\
\hline Sulfur & $5 \times 10^{-4}$ & 2 & B.2.2.4 & & & \\
\hline Zinc & $2 \times 10^{-5}$ & 2 & B.2.2.5 & $3 \times 10^{-5}$ & 1 & B.2.3.8 \\
\hline Lead & $2 \times 10^{-5}$ & 2 & B.2.2.6 & $2 \times 10^{-4}$ & 1 & B.2.3.22 \\
\hline Copper & & & & $1 \times 10^{-4}$ & $?$ & B.2.3.1 \\
\hline Silica & & & & $2 \times 10^{-2}$ & 1 & B.2.3.2 \\
\hline Fluoride & & & & $1 \times 10^{-3}$ & 1 & B.2.3.3 \\
\hline Iron & & & & $2 \times 10^{-4}$ & 1 & B.2.3.4 \\
\hline Chloride & & & & 2 & 1 & $B .2 .3 .5$ \\
\hline Magnes ium & & & & $2 \times 10^{-2}$ & 1 & B.2.3.6 \\
\hline Calcium & & & & $4 \times 10^{-2}$ & 1 & B.2.3.7 \\
\hline Phosphate & & & & $2 \times 10^{-4}$ & 1 & B.2.3.9 \\
\hline Nitrate & & & & $1 \times 10^{-2}$ & 1 & B.2.3.10 \\
\hline Sulfates & & & & $3 \times 10^{-1}$ & 1 & B.2.3.11 \\
\hline Sodium & & & & 1 & 1 & B.2.3.12 \\
\hline Ammonia & & & & $1 \times 10^{-3}$ & 1 & B.2.3.13 \\
\hline Kjeldahl Nitrogen & & & & $5 \times 10^{-2}$ & 1 & B.2.3.14 \\
\hline Aluminum & & & & $5 \times 10^{-4}$ & 1 & B.2.3.15 \\
\hline Titanium & & & & $1 \times 10^{-3}$ & 1 & B.2.3.17 \\
\hline Arsenic & & & & $2 \times 10^{-5}$ & 1 & B. 2.3 .18 \\
\hline Molybdenum & & & & $9 \times 10^{-4}$ & 1 & B.2.3.19 \\
\hline Vanadium & & & & $1 \times 10^{-3}$ & 1 & B.2.3.20 \\
\hline Manganese & & & & $3 \times 10^{-4}$ & 1 & B.2.3.21 \\
\hline
\end{tabular}




\section{B.2 UNDERGROUND MINE CALCULATIONS}

\section{B.2.1 ASSUMPTIONS}

The following values were previously calculated in Appendix $A^{(1)}$ and will be used extensively in the subsequent calculations.

$\begin{array}{cc}\text { Value } & \frac{\text { Calc. }}{2-7(1)} \\ \begin{array}{c}6351 \mathrm{~kg} \mathrm{U}(\text { input }) / \text { day } \\ 1.87 \times 10^{6} \mathrm{ga} \text { mine water to surface } \\ \text { runoff/day }\end{array} & 2-7(1)\end{array}$

Fresh air enters the seven mines at a total rate of $6.26 \times 10^{10} \mathrm{z} /$ day. It is assumed that air also discharges at this rate. At the average elevation of $2134 \mathrm{~m}(7000 \mathrm{ft})$, this air discharge would weigh about $6.22 \times 10^{7} \mathrm{~kg}$ day (see calculation B.1.3.23, Appendix B).

The Rio Algon Uranium Mine reported the 1 iquid discharge concentrations used for calculations B.1.3.1 to B.1.3.12. This water was discharged from the ventilation shaft. Release factors are calculated assuming concentrations are also the same for water discharged from the production shaft.

Reference 5 makes no distinction between underground and surface mine waste water because choice of mine is determined by factors other than chemical characteristics of the deposits. In lieu of this reasoning, data from Reference 5 is used for release factor calculations B.1.3.15 to B.1.3.22 since data specific to underground mine water is not available for these elements. (When a range of concentrations is given, the median is used for calculation.)

Several other elemental airborne effluents, in addition to those calculated in this report, are present in the mine-air discharge. However, it is assumed that these particles are considered in the release factor for rock dust. (1) Beryllium and cadmium (Calculations B.1.2.2, B.1.2.3) are considered separately from rock dust because of their toxicity. 


\section{B.2.2 AIRBORNE RELEASES}

B.2.2.1 Benzo(a)pyrene - resulis from incomplete combustion of diesel fuel

$$
\begin{aligned}
& (2, \text { p. 4-6) } \\
& 62 \frac{2 \mathrm{~g} \mathrm{BaP}}{\text { gal fueT }} \times 7000 \text { gal fuel/day } \times \frac{\mathrm{kg}}{10^{9} \mathrm{\mu g}}=4.34 \times 10^{-4} \mathrm{~kg} \mathrm{BaP} / \text { day } \\
& \therefore \frac{4.34 \times 10^{-4} \mathrm{~kg} \mathrm{BaP} / \text { day }}{6351 \mathrm{~kg} \mathrm{U} / \text { day }}=6.8 \times 10^{-8} \frac{\mathrm{kg} \mathrm{BaP}}{\mathrm{kg} \mathrm{U} \text { (input) }}
\end{aligned}
$$

B.2.2.2 Beryllium - Beryllium and cadmium will be considered separately from rock dust because of their toxicity.

(2, p. 5-42)

$\frac{100 \mathrm{ng}}{\mathrm{m}^{3}} \times 6.26 \times 10^{7} \mathrm{~m}^{3} /$ day $\times \frac{\mathrm{kg}}{10^{12} \mathrm{ng}}=6.3 \times 10^{-3} \mathrm{Kg} \mathrm{Be} /$ day

$\therefore \frac{6.3 \times 10^{-3} \mathrm{~kg} \mathrm{Be} / \text { day }}{6351 \mathrm{~kg} \mathrm{U} / \text { day }}=9.9 \times 10^{-7} \frac{\mathrm{kg} \mathrm{Be}}{\mathrm{kgU \text {(input) }}}$

\section{B.2.2.3 Cadmium}

(2, p. 5-42)

$\frac{200 \mathrm{ng}}{\mathrm{m}^{3}} \times 6.26 \times 10^{7} \mathrm{~m}^{3} /$ day $\times \frac{\mathrm{kg}}{10^{12} \mathrm{ng}}=.0125 \mathrm{~kg} \mathrm{Cd} /$ day

$\therefore \frac{.0125 \mathrm{~kg} \mathrm{Cd} / \text { day }}{6351 \mathrm{~kg} \mathrm{U} / \text { day }}=1.97 \times 10^{-6} \frac{\mathrm{kg} \mathrm{Cd}}{\mathrm{kg} \mathrm{U} \text { (input) }}$

B.2.2.4 Sulfur - from blasting, fuses probable source

(2, p. 5-41)

$6.26 \times 10^{7} \mathrm{~m}^{3} /$ day $\times \frac{50 \mu \mathrm{g}}{\mathrm{m}^{3}} \times \frac{\mathrm{kg}}{10^{9} \mu \mathrm{g}}=3.13 \mathrm{~kg} \mathrm{~S} /$ day

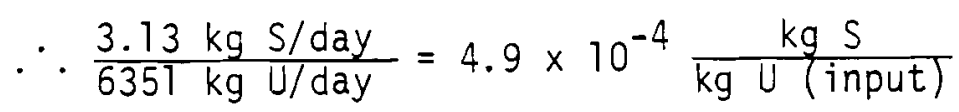


B.2.2.5 Zinc-blasting, blasting caps source

(2, p. 5-41)

$\frac{2 \mathrm{Lg}}{\mathrm{m}^{3}} \times 6.26 \times 10^{7} \mathrm{~m}^{3} /$ day $\times \frac{\mathrm{kg}}{10^{9} \mu \mathrm{g}}=.125 \mathrm{~kg} \mathrm{Zn/ \text {day }}$

$\therefore \frac{.125 \mathrm{~kg} \mathrm{Zn} / \mathrm{day}}{6351 \mathrm{kgU} / \mathrm{day}}=1.97 \times 10^{-5} \frac{\mathrm{kg} \mathrm{Zn}}{\mathrm{kgU}(\text { input })}$

B.2.2.6 Lead - blasting, blasting caps source

See Calculation B.2.2.5 for Zinc.

\section{B.2.3 LIQUID RELEASES}

\section{B.2.3.1 Copper}

(3, p. 38)

$.1 \mathrm{mg} / \mathrm{l} \times \frac{\mathrm{kg}}{10^{6} \mathrm{mg}} \times 3.785 \mathrm{l} / \mathrm{ga} 1 \times 1.87 \times 10^{6} \mathrm{gal} /$ day $=.71 \mathrm{~kg} \mathrm{Cu} /$ day

$\therefore \frac{.71 \mathrm{~kg} \mathrm{Cu} / \text { day }}{6351 \mathrm{~kg} \mathrm{U} / \text { day }}=1.1 \times 10^{-4} \frac{\mathrm{kg} \mathrm{Cu}}{\mathrm{kgU}(\text { input })}$

\section{B.2.3.2 Silica}

$(3$, p. 38)

$14.2 \mathrm{mg} / \mathrm{l} \times \frac{\mathrm{kg}}{10^{6} \mathrm{mg}} \times 3.785 \mathrm{l} / \mathrm{ga} 1 \times 1.87 \times 10^{6} \mathrm{gal} /$ day

$=1.0 \times 10^{2} \mathrm{~kg} \mathrm{silica} /$ day

$\therefore \frac{1 \times 10^{2} \mathrm{~kg} \mathrm{silica/day}}{6351 \mathrm{~kg} \mathrm{U} / \text { day }}=1.6 \times 10^{-2} \frac{\mathrm{kg} \text { silica }}{\mathrm{kgU} \text { (input) }}$

\section{B.2.3.3 Fluoride}

$(3$, p. 38)

$1.28 \mathrm{mg} / \ell \times \frac{\mathrm{kg}}{10^{6} \mathrm{mg}} \times 3.785 \mathrm{\ell} / \mathrm{gal} \times 1.87 \times 10^{6} \mathrm{ga} 1 / \mathrm{day}$ 


$$
\begin{aligned}
& =9.05 \mathrm{~kg} \text { fiuoride/day } \\
& \therefore \frac{9.06 \mathrm{~kg} \mathrm{fiuoride} / \mathrm{day}}{6351 \mathrm{~kg} \mathrm{~J} / \mathrm{day}}=1.4 \times 10^{-3} \frac{\mathrm{kg} \text { fluoride }}{\mathrm{kg} \mathrm{U} \text { (input) }}
\end{aligned}
$$

B.2.3.4 Iron

$$
\begin{aligned}
& \text { (3, p. 38) } \\
& .16 \mathrm{mg} / 2 \times \frac{\mathrm{kg}}{10^{6} \mathrm{mg}} \times 3.785 \mathrm{\ell} / \mathrm{ga} 1 \times 1.87 \times 10^{6} \mathrm{ga} 1 / \text { day }=1.13 \mathrm{~kg} \mathrm{Fe} / \text { day } \\
& \therefore \frac{1.13 \mathrm{~kg} \mathrm{Fe} / \text { day }}{6351 \mathrm{~kg} \mathrm{U} / \text { day }}=1.78 \times 10^{-4} \frac{\mathrm{kg} \mathrm{Fe}}{\mathrm{kg} \mathrm{U} \text { (input) }}
\end{aligned}
$$

\section{B.2.3.5 Chloride}

(3, p. 38)

$1597 \mathrm{mg} / 2 \times \frac{\mathrm{kg}}{10^{6} \mathrm{mg}} \times 3.785 \mathrm{\ell} / \mathrm{gal} \times 1.87 \times 10^{6} \mathrm{gal} /$ day

$=1.13 \times 10^{4} \mathrm{~kg}$ chloride $/$ day

$\therefore \frac{1.13 \times 10^{4} \mathrm{~kg} \mathrm{chloride} \mathrm{day}}{6351 \mathrm{~kg} \mathrm{U} / \text { day }}=1.78$

\section{B.2.3.6 Magnesium}

$(3, p .38)$

$17.2 \mathrm{mg} / 2 \times \frac{\mathrm{kg}}{10^{6} \mathrm{mg}} \times 3.785 \mathrm{l} / \mathrm{ga} 1 \times 1.87 \times 10^{6} \mathrm{gal} / \mathrm{day}$

$=1.22 \times 10^{2} \mathrm{~kg} \mathrm{Mg} / \mathrm{day}$

$\therefore \frac{1.22 \times 10^{2} \mathrm{~kg} \mathrm{Mg} / \text { day }}{6351 \mathrm{~kg} \mathrm{U} / \text { day }}=\frac{1.9 \times 10^{-2} \mathrm{~kg} \mathrm{Mg}}{\mathrm{kgU} \text { (input) }}$

\section{B.2.3.7 Calcium}

(3, p. 38) 
$37.8 \mathrm{mg} \mathrm{Ca} / \mathrm{i} \times \frac{\mathrm{kg}}{10^{6} \mathrm{mg}} \times 3.785 \mathrm{z} / \mathrm{gal} \times 1.87 \times 10^{6} \mathrm{ga} 1 / \mathrm{day}=268 \mathrm{~kg} \mathrm{Ca} / \mathrm{day}$ $\therefore \frac{268 \mathrm{~kg} \mathrm{Ca} / \text { day }}{6351 \mathrm{~kg} \mathrm{U} / \text { day }}=4.2 \times 10^{-2} \frac{\mathrm{kg} \mathrm{Ca}}{\mathrm{kg} \mathrm{U} \text { (input) }}$

\section{B.2.3.8 Zinc}

(3, p. 38)

$.03 \mathrm{mg} / 2 \times \frac{\mathrm{kg}}{10^{6} \mathrm{mg}} \times 3.785 \mathrm{\ell} / \mathrm{gal} \times 1.87 \times 10^{6} \mathrm{gal} / \mathrm{day}=.21 \mathrm{~kg} \mathrm{Zn/day}$

$\therefore \frac{.21 \mathrm{~kg} \mathrm{Zn} / \mathrm{day}}{6351 \mathrm{~kg} \mathrm{U} / \mathrm{day}}=3.3 \times 10^{-5} \frac{\mathrm{kg} \mathrm{Zn}}{\mathrm{kgU} \text { (input) }}$

\section{B.2.3.9 Phosphate}

(3, p. 38)

$.2 \mathrm{mg} / \ell \times \frac{\mathrm{kg}}{10^{6} \mathrm{mg}} \times 3.785 \mathrm{\ell} / \mathrm{gal} \times 1.87 \times 10^{6} \mathrm{gal} /$ day $=1.4 \mathrm{~kg} \mathrm{PO} / /$ day

$\therefore \frac{1.4 \mathrm{~kg} \mathrm{PO} / \text { day }}{6351 \mathrm{~kg} \mathrm{U/day}}=2.20 \times 10^{-4} \frac{\mathrm{kg} \mathrm{PO}_{4}}{\mathrm{~kg} \mathrm{U} \text { (input) }}$

\section{B.2.3.10 Nitrates}

$(3, p .38)$

$9.5 \mathrm{mg} / \ell \times \frac{\mathrm{kg}}{10^{6} \mathrm{mg}} \times 3.785 \mathrm{\ell} / \mathrm{ga} 7 \times 1.87 \times 10^{6} \mathrm{gal} /$ day $=67.2 \mathrm{~kg} \mathrm{NO}_{3} /$ day

$\therefore \frac{67.2 \mathrm{~kg} \mathrm{NO}_{3} / \text { day }}{6351 \mathrm{~kg} \mathrm{U/ \text {day }}}=1.0 \times 10^{-2} \frac{\mathrm{kg} \mathrm{NO}_{3}}{\mathrm{~kg} \mathrm{U} \text { (input) }}$

\section{B.2.3.11 Sulfates}

(3, p. 38)

$309 \mathrm{mg} / \ell \times \frac{\mathrm{kg}}{10^{6} \mathrm{mg}} \times 3.785 \mathrm{\ell} / \mathrm{gal} \times 1.87 \times 10^{6} \mathrm{gal} / \mathrm{day}$

$=2.19 \times 10^{3} \mathrm{~kg} \mathrm{SO}_{4} /$ day

$\therefore \frac{2.19 \times 10^{3} \mathrm{~kg} \mathrm{SO}_{4} / \text { day }}{6351 \times \mathrm{kg} \mathrm{U} / \mathrm{day}}=3.4 \times 10^{-1} \frac{\mathrm{kg} \mathrm{SO}_{4}}{\mathrm{~kg} \mathrm{U} \text { (input) }}$ 


\section{B.2.3.12 Sodium}

(3. p. 30)

$1335 \mathrm{mg} /: \times \frac{\mathrm{kg}}{10^{6} \mathrm{mg}} \times 3.735 \mathrm{i} / \mathrm{gal} \times 1.37 \times 10^{6} \mathrm{gal} / \mathrm{day}=9.410^{3} \mathrm{~kg} \mathrm{ia} / \mathrm{day}$

$\therefore \frac{9.4 \times 10^{3} \mathrm{~kg} \mathrm{fla} / \text { day }}{6351 \mathrm{~kg} \mathrm{U} / \text { day }}=1.5 \frac{\mathrm{kg} \mathrm{Na}}{\mathrm{kg} \mathrm{U}(\text { input })}$

\section{B.2.3.13 Ammonia}

(4. p. A-13)

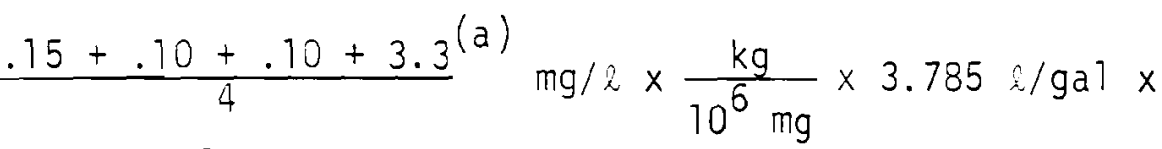

$1.87 \times 10^{6} \mathrm{ga} 1 / \mathrm{day}=6.5 \mathrm{~kg} \mathrm{iHH}_{3} / \mathrm{day}$

$\therefore \frac{6.5 \mathrm{~kg} \mathrm{NH} / \text { day }}{6351 \mathrm{~kg} \mathrm{U} / \text { day }}=1.0 \times 10^{-3} \frac{\mathrm{kg} \mathrm{NH}_{3}}{\mathrm{kgU} \text { (input) }}$

\section{B.2.3.14 Kjeldahl Nitrogen}

(4, P. A-13)

$\left.\frac{.55+145+.3+21.8^{(\mathrm{a})}}{4} \mathrm{mg} / \ell \times \frac{\mathrm{kg}}{10^{6} \mathrm{mg}} \times 3.785 \mathrm{\ell} / \mathrm{ga}\right] \times 1.87 \times 10^{6} \mathrm{ga} / \mathrm{day}$

$=3 \times 10^{2} \mathrm{~kg} \mathrm{~N} / \mathrm{day}$

$\therefore \frac{300 \mathrm{~kg} \mathrm{~N} / \text { day }}{6351 \mathrm{~kg} \mathrm{U} / \mathrm{day}}=4.7 \times 10^{-2} \frac{\mathrm{kg} \mathrm{iN}}{\mathrm{kg} \mathrm{U} \text { (input) }}$

\section{B.2.3.15 Aluminum}

(5, p. 336)

$.45 \mathrm{mg} / \ell \times 1.87 \times 10^{6} \mathrm{gal} /$ day $\times 3.785 \mathrm{2} / \mathrm{ga} 1 \times \frac{\mathrm{kg}}{10^{6} \mathrm{mg}}=3.18 \mathrm{~kg} \mathrm{~A} 1 /$ day

$\therefore \frac{3.18 \mathrm{~kg} \mathrm{Al} / \text { day }}{6351 \mathrm{~kg} \mathrm{U} / \mathrm{day}}=5.0 \times 10^{-4} \frac{\mathrm{kg} \mathrm{Al}}{\mathrm{kg} \mathrm{U}}$

(a) Concentration data from 4 mines in Colorado. 


\subsubsection{Beryllium}

(5, p. 336)

$.01 \mathrm{mg} / 2 \times 1.87 \times 10^{6} \mathrm{gai} /$ day $\times \frac{\mathrm{kg}}{10^{6} \mathrm{mg}} \times 3.7852 / \mathrm{gal}=.07 \mathrm{~kg} \mathrm{Be} /$ day

$\therefore \frac{.07 \mathrm{~kg} \mathrm{Be} / \text { day }}{6357 \mathrm{~kg} \mathrm{U} / \mathrm{day}}=1.1 \times 10^{-5} \frac{\mathrm{kg} \mathrm{Be}}{\mathrm{kg} \mathrm{U}}$

B.2.3.17 Titanium

(5, p. 336)

$.95 \mathrm{mg} / \ell \times 1.87 \times 10^{6} \mathrm{gal} /$ day $\times 3.785 \mathrm{l} / \mathrm{gal} \times \frac{\mathrm{kg}}{10^{6} \mathrm{mg}}=6.72 \mathrm{~kg} \mathrm{Ti} /$ day

$\therefore \frac{6.72 \mathrm{~kg} \mathrm{Ti} / \text { day }}{6351 \mathrm{~kg} \mathrm{U} / \text { day }}=1.1 \times 10^{-3} \frac{\mathrm{kg} \mathrm{Ti}}{\mathrm{kg} \mathrm{U}}$

B.2.3.18 Arsenic

(5, p. 336)

$.02 \mathrm{mg} / \ell \times 1.87 \times 10^{6} \mathrm{gal} /$ day $\times 3.785 \mathrm{l} / \mathrm{ga} 1 \times \frac{\mathrm{kg}}{10^{6} \mathrm{mg}}=.14 \mathrm{~kg} \mathrm{As} /$ day

$\therefore \frac{.14 \mathrm{~kg} \mathrm{As} / \mathrm{day}}{6351 \mathrm{~kg} \mathrm{U} / \mathrm{day}}=2.2 \times 10^{-5} \frac{\mathrm{kg} \mathrm{As}}{\mathrm{kg} \mathrm{U}}$

\section{B.2.3.19 Molybdenum}

(5, p. 336)

$.85 \mathrm{mg} / \mathrm{l} \times 1.87 \times 10^{6} \mathrm{gal} /$ day $\times 3.785 \mathrm{l} / \mathrm{gal} \times \frac{\mathrm{kg}}{10^{6} \mathrm{mg}}=6.02 \mathrm{~kg} \mathrm{Mo} / \mathrm{day}$

$\therefore \frac{6.02 \mathrm{~kg} \mathrm{Mo} / \text { day }}{6351 \mathrm{~kg} \mathrm{U} / \mathrm{day}}=9.5 \times 10^{-4} \frac{\mathrm{kg} \mathrm{M}: 10}{\mathrm{~kg} \mathrm{U}}$

\section{B.2.3.20 Vanadium}

$(6, p .11)$

$1.3 \mathrm{mg} / \ell \times 1.87 \times 10^{6} \mathrm{gal} /$ day $\times 3.785 \mathrm{\ell} / \mathrm{gal} \times \frac{\mathrm{kg}}{10^{6} \mathrm{mg}}=9.2 \mathrm{~kg} \mathrm{~V} /$ day

$\therefore \frac{9.2 \mathrm{~kg} \mathrm{~V} / \text { day }}{6357 \mathrm{~kg} \mathrm{U} / \text { day }}=1.4 \times 10^{-3} \frac{\mathrm{kg} \mathrm{V}}{\mathrm{kgU( \text {input } )}}$ 


\section{B.2.3.21 Manganese}

$$
\begin{aligned}
& \text { (5, p. 336) } \\
& .25 \mathrm{mg} /: \times 1.87 \times 10^{6} \mathrm{ga} 1 / \mathrm{day} \times 3.785 \mathrm{i} / \mathrm{gal} \times \frac{\mathrm{kg}}{10^{5} \mathrm{mg}}=1.77 \mathrm{~kg} \mathrm{Mn} / \mathrm{day} \\
& \therefore \frac{1.77 \mathrm{~kg} \mathrm{Mn} / \mathrm{day}}{6351 \mathrm{~kg} \mathrm{U} / \mathrm{day}}=2.8 \times 10^{-4} \frac{\mathrm{kg} \mathrm{Mn}}{\mathrm{kg} \mathrm{U}(\text { input) }}
\end{aligned}
$$

\section{B.2.3.22 Lead}

From Appendix A, page 11, water discharged to surface is estimated at $1.87 \times 10^{6} \frac{\mathrm{gal}}{\mathrm{day}}$ and reference 5 , p. 337 :

$$
\begin{aligned}
& .2 \mathrm{mg} / 2 \times 1.87 \times 10^{6} \mathrm{gal} / \text { day } \times 3.785 \mathrm{\ell} / \mathrm{ga} 1 \times \frac{\mathrm{kg}}{10^{6} \mathrm{mg}}=1.42 \mathrm{~kg} \mathrm{~Pb} / \text { day } \\
& \therefore \frac{1.42 \mathrm{~kg} \mathrm{~Pb} / \text { day }}{6351 \mathrm{kgU} / \text { day }}=2.23 \times 10^{-4} \frac{\mathrm{kg} \mathrm{Pb}}{\mathrm{kg} \mathrm{U}}
\end{aligned}
$$

\section{B.2.3.23 Fresh Air}

Fresh air is pumped through al1 work areas of the mines so that surplus oxygen is available to the miners and radioactive and chemical pollutants are maintained within safe limits. Walter I. Enderlin, at PNL, obtained from KerrMcGee's Ambrosia Lake, New Mexico facilities an average of 206,000 CFM of fresh air for each of the seven mines at average elevation 2134 meters or 7000 feet:

$0.206 \times 10^{6} \mathrm{CFM} \times 28.32 \frac{\ell}{\mathrm{CF}} \times 1440 \frac{\text { minutes }}{\text { day }} \times 7$ mines $=5.88 \times 10^{10} \frac{\ell}{\text { day }}$ fresh air

Our reference underground facility is assumed to be $6-1 / 2 \%$ larger than the Ambrosia Lake mines so that the contained uranium produced in the mined ore is the same quantity as that produced from the reference surface mine. This makes all effluents directly comparable. Since:

$$
1.065 \times 5.88 \times 10^{10} \frac{\ell}{\text { day }}=6.26 \times 10^{10} \frac{\ell}{\text { day }} \text { fresh air }
$$


Density ratio for air from page 1530, ilarks' Handbook, Fourth Edition, 1941 at 6000 feet $=0.3358,3000$ feet $=0.7859$,

the interpolated value for 7000 feet $=0.81085$;

The weight of air at sea level, $760 \mathrm{~mm} \mathrm{Hg}, 15^{\circ} \mathrm{C}\left(59^{\circ} \mathrm{F}\right)=1.2256 \frac{\mathrm{g}}{\mathrm{l}}$ (from page 470, Standard Handbook for Electrical Engineers, Seventh Edition, 1941)

then $6.26 \times 10^{10} \frac{\ell}{\text { day }}$ fresh air $\times 1.2256 \frac{\mathrm{g}}{\ell} \times 0.81085=6.22 \times 10^{10} \frac{\mathrm{g}}{\mathrm{day}}$ or $6.22 \times 10^{7} \frac{\mathrm{kg}}{\text { day }}$ of fresh air.

TABLE B-3. Acid Leach $11 i 11$ Chemical Release Factors $\mathrm{kg} / \mathrm{kg} \cup$ (input)

\begin{tabular}{|c|c|c|c|c|c|c|}
\hline \multirow[b]{2}{*}{ Material Released } & \multicolumn{3}{|c|}{ Airborne } & \multicolumn{3}{|c|}{ Liquid } \\
\hline & Factor & Reliability & $\begin{array}{l}\text { Ref. } \\
\text { Calc. }\end{array}$ & Factor & Reliability & $\begin{array}{l}\text { Ref. } \\
\text { Calc. }\end{array}$ \\
\hline Tertiary Amine & & & & $1 \times 10^{-3}$ & 2 & B. 3.3 .1 \\
\hline Isodecanol & & & & $2 \times 10^{-3}$ & 2 & B.3.3.2 \\
\hline Kerosene & $9 \cdot 10^{-2}$ & 2 & B.3.3.3 & & & \\
\hline Beryllium & & & & $1 \times 10^{-5}$ & 2 & B.3.3.4 \\
\hline Chromium & & & & $9 \times 10^{-4}$ & 2 & B. 3.3 .5 \\
\hline Molybdenum & & & & $1 \times 10^{-3}$ & 2 & B.3.3.6 \\
\hline Copper & & & & $3 \times 10^{-4}$ & 2 & B.3.3.7 \\
\hline ivickel & & & & $2 \times 10^{-4}$ & 2 & B. 3.3 .8 \\
\hline Lead & & & & $2 \times 10^{-4}$ & 2 & B. 3.3 .9 \\
\hline Zine & & & & $5 \times 10^{-4}$ & 2 & B. 3.3 .10 \\
\hline Titanium & & & & $1 \times 10^{-3}$ & 2 & B.3.3.11 \\
\hline Sulfide & & & & $4 \times 10^{-5}$ & 2 & $8.3 .3 \cdot 12$ \\
\hline Cyanide & & & & $8 \times 10^{-7}$ & 2 & B. 3.3 .13 \\
\hline Silver & & & & $8 \times 10^{-7}$ & 2 & B. 3.3 .14 \\
\hline Manganese & & & & $2 \times 10^{-2}$ & 2 & B. 3.3 .15 \\
\hline Magnesium & & & & $9 \times 10^{-2}$ & 2 & B. 3.3 .16 \\
\hline Seleni um & & & & 0 & 2 & 8.3 .3 .17 \\
\hline Sulfuric Acid & $n e g^{(a)}$ & 2 & & $8 \times 10^{-2}$ & $i$ & B. 3.3 .18 \\
\hline Arsine & 0 & 1 & & & & \\
\hline Stibine & 0 & 1 & & & & \\
\hline Hydrogen Sulfide & neg (a) & 1 & & & & \\
\hline
\end{tabular}

(a) negligible 
• 


\section{B.3 ACID LEACH ILL CALCULATIONS}

\section{B.3.1 ASSLMPTIONS}

The following values will be used extensively in the succeeding calculations:

From Appendix A

$2722 \mathrm{~kg} \cup$ (input)/day Calculation A.3-1

$80 \mathrm{gal} / \mathrm{min}$ pond flow out Calculation A.3-2

$500 \mathrm{gal} / \mathrm{min}$ overflow into pond Calculation A.3-2

The reference facility has a tailings pond behind a dam which receives all liquid effluents from the mill. It is amply large so that: (a)

1. Effluents totaling $500 \mathrm{gpm}$ can flow into it 24 hours per day, 365 days per year.

2. Sufficient extra capacity is available to receive the $500 \mathrm{gpm}$ during sub-zero weather which may last several months.

3. Allowance is made for buildup of sludges on the bottom of the pond; sludge will occupy more space with the passage of time.

It is expected that there will initially be a maximum seepage of $80 \mathrm{gpm}$ of tailing solution into the sides of the tailing basin. $(10 \mathrm{p} .37)$ It is also expected that sludges, etc., will gradually seal off the seepage, so that after about five years there will be no seepage. Mill operations will try to maintain 7 or 8 feed of "freeboard" at al1 times so that the dam will never be overtopped.

According to Reference 5 p. 165, in a general analysis of raw waste water, the only distinction that must be made is between mills using acid leaching of ore and mills using alkaline leaching of ore. "While other differences between ores and processes can have an effect on waste water characteristics, they are not believed to justify further subcategorization. "(5) Therefore the data on

(a) Information supplied by process engineers at Highland Uranium Mill near Casper, Wyoming. 
mi11 wastes presented in this report, despite the fact that it is noz specific to the EifFORi model, is assumed to be apolicable for release factor calculations. (See Caiculations B.3.3. T to B.3.3.15.)

\section{B.3.2 AIRBORINE RELEASES}

The leach tank vent system releases small amounts of sulfur dioxide, sulfuric acid fumes $\left(\mathrm{SO}_{3}\right)$, and possibly hydrogen sulfide. According to the Department of Energy $(4, p . B-10)$ and their Grand Junction office ${ }^{(8)}$, the concentration of these gases is very low. In the case of the sulfur oxides, the amount released in the leach tank vents is negligible in comparison with the oxides released as combustion products, therefore they are neglected. Hydrogen sulfide has been detected only in the leaching of high sulfide ore. Since most uranium ore in the United States contains little, if any, sulfur, ( $\ddot{u}$ ) hydrogen sulfide is also neglected.

Arsine $\left(\mathrm{AsH}_{3}\right)$ and stibine $\left(\mathrm{SbH}_{3}\right)$ have also been cited as possible effluents from the leach tank vents in the milling of ores containing arsenic and antimony. (11) Both compounds result from reduction in an acid solution of other antimony or arsenic compounds in uranium ore. Since both gases are unstable towards heat (1each tank temperature approximately $95^{\circ} \mathrm{F}$ ), the compounds (if formed) probably ciecompose and wil1 be neglected in our study. If future evidence indicates the existence of arsine and stibine, re-examination of these gases may be necessary.

\section{B.3.3 LIQUID RELEASES}

From Reference 6 p. 192, we learn that "Most reagents used in present commercial operations are relatively stable and have low volatility." We therefore assume no tertiary amine or isodecanol in the gaseous effluents. "Entrainment losses usually result from the presence of very finely dispersed organic in the aqueous phase, often due either to excessive intensity in mixing, or to insufficient capacity in the settlers and incomplete phase separation...." We therefore are assuming both the tertiary amine and isodecanol as being contained in the liquid effluents only. 


\section{B.3.3.1 Tertiary Amine (AR 2}

ECT document "6.0 Uranium Mi11ing", derives an input of $2722 \mathrm{~kg} \mathrm{U/day:}$

$2722 \mathrm{~kg} \mathrm{U} /$ day $\times 93.5$. recovered $\times \frac{842.082 \text { atomic } w t . U_{3} 0_{3}}{714.087 \text { atomic } w t . ~} 3$

$=3001 \mathrm{~kg} \mathrm{U} \mathrm{O}_{8}$ produced/day

(6, p. 192)

$\frac{.008 \mathrm{~kg} \mathrm{NR}}{\mathrm{kg} \mathrm{U} \mathrm{U}_{3} \text { produced }} \times 3007 \mathrm{~kg} \mathrm{U} \mathrm{U}_{3} \mathrm{O}_{8}$ produced $/$ day $=24.0 \mathrm{~kg} \mathrm{NR} /$ day

$24.0 \mathrm{~kg} \mathrm{NR}{ }_{3} /$ day $\times \frac{80 \mathrm{ga} 7 / \mathrm{min}}{500 \mathrm{ga} 1 / \mathrm{min}}=3.84 \mathrm{~kg} \mathrm{NR}_{3} /$ day

$\therefore \frac{3.84 \mathrm{~kg} \mathrm{NR} / \text { day }}{2722 \mathrm{~kg} \text { U/day }}=1.41 \times 10^{-3} \frac{\mathrm{kg} \mathrm{NR}}{\mathrm{kgU}(\text { input })}$

\section{B.3.3.2 Isodecanol (a long-chain alcohol)}

From engineers at the Highland Mill we learned that their organic solvent is composed of the following:

$$
\begin{aligned}
2 \% & \text { isodecanol } \\
3 \% & \text { Tertiary amine } \\
95 \% & \text { kerosene }
\end{aligned}
$$

According to R. C. Merritt(6 p. 192), "the loss of isodecanol will be nearly twice as great in proportion to its concentration in the solvent as the loss of amine."

From the foregoing tertiary amine calculation we have $24: 0 \mathrm{~kg}$ of $\mathrm{NR}_{3} /$ day:

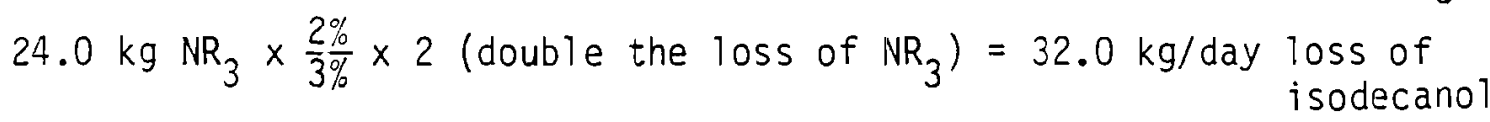

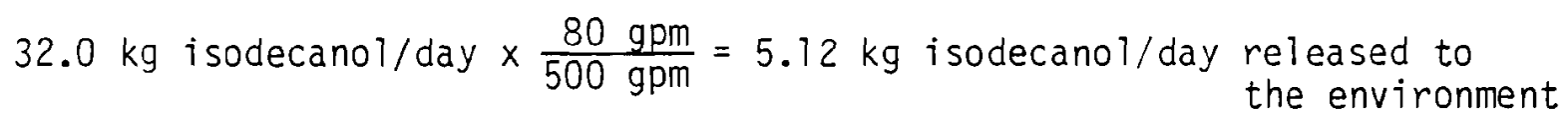

$\frac{5.12 \mathrm{~kg} \text { isodecanol/day released }}{2722 \mathrm{~kg} \mathrm{U} / \text { day }}=1.88 \times 10^{-3} \frac{\mathrm{kg} \text { isodecanol }}{\mathrm{kg} \mathrm{U} \text { (input) }}$ release factor 


\section{B.3.3.3 Kerosene}

Reference 1 calculated an airborne release factor of $2 \times 10^{-4} \frac{\mathrm{kg}}{\mathrm{kg}} \frac{\mathrm{ker} \text { esene }}{\mathrm{U} \text { input }}$. This is for the uranium acid leach mill itself, vented through the roof stack. There is a liquid release which originates from the two bieed streams shown on Figure 7.2, page 7-5 of Section 7 . This 1 iquid release is unusual in that the kerosene discarded as part of these waste streams flows out to the tailings pond where it floats on top of the water and approximately 100\% of it eventually becomes an airborne release. In warm or cold weather the Kerosene floats on the water and gradually evaporates. In extremely cold weather the raffinate bleed streams flow onto the top of the ice, and again, the kerosene component gradually evaporates. Although this kerosene in the bleed streams is initially a liquid effluent, it is eventually an airborne effluent released to the environment.

Reference 8 says about 0.1 liter of kerosene is lost to the bleed stream (Figure 7.2) per 1,000 1iters of aqueous solution circulated in the solvent extraction step.

The specific gravity of kerosene is assumed to be 0.82 .

Reference 6 , page 137, assumes one gram $U_{3} 0_{8}$ needs one liter of aqueous feed, or 1000 \& per $\mathrm{kg}$ of $\mathrm{U}_{3} \mathrm{O}_{8}$.

The ratio of the weights of $U_{3} O_{8}$ to $U$ is $\frac{.18}{.15}$.

$2722 \mathrm{~kg} \mathrm{U} /$ day $\times 0.95 \mathrm{U}$

in ore is soluble and reaches solvent extraction step $\times \frac{0.18 \mathrm{~g} \mathrm{U}_{3} \mathrm{O}_{8}}{0.15 \mathrm{~g} \mathrm{U}}$

$\times \frac{1000 \ell \text { aqueous solvent circulated }}{\mathrm{kg} \mathrm{U} \mathrm{O}_{3} \mathrm{O}} \times \frac{0.11 \mathrm{iter} \text { lost of bleeds }}{1000 \text { l aqueous circulated }} \times \frac{0.82 \mathrm{~kg}}{\ell}$ density

$=254 \mathrm{~kg} /$ day kerosene list as part of liquid effluent to tailings ponds, approximately $100 \%$ of which eventually becomes an airborne effluent.

$\therefore \frac{254 \mathrm{~kg} \mathrm{kerosene} / \text { day }}{2722 \mathrm{~kg} \mathrm{U} / \text { day }}=9.3 \times 10^{-2} \frac{\mathrm{kg} \text { kerosene (airborne) }}{\mathrm{kgU} \text { (input) }}$

\section{B.3.3.4 Beryllium}

$(5, \mathrm{p}, 336)$

$.08 \mathrm{mg} / \mathrm{l} \times 80 \mathrm{gal} / \mathrm{min} \times 1440 \mathrm{~min} /$ day $\times 3.785 \mathrm{l} / \mathrm{gal} \times \frac{\mathrm{kg}}{10^{6} \mathrm{mg}}=.035 \mathrm{~kg}$

$\mathrm{Be} / \mathrm{day} \therefore \frac{.035 \mathrm{~kg} \mathrm{Be} / \text { day }}{2722 \mathrm{~kg} \mathrm{U} / \mathrm{day}}=1.3 \times 10^{-5} \frac{\mathrm{kg} \mathrm{Be}}{\mathrm{kg} \mathrm{U}}$ 
$\Xi .3 .3 .5$ Chronium

$$
\begin{aligned}
& (5, \mathrm{p.} 336) \\
& \left.\left(\frac{2+g}{2}\right) \mathrm{mg} / \hat{\imath} \times 80 \mathrm{ga} / \mathrm{min} \times 1440 \mathrm{~min} / \mathrm{day} \times 3.785 \mathrm{l} / \mathrm{ga}\right] \times \frac{\mathrm{kg}}{106 \mathrm{mg}} \\
& =2.398 \mathrm{~kg} \mathrm{Cr} / \mathrm{day} \\
& \therefore \frac{2.398 \mathrm{~kg} \mathrm{Cr} / \mathrm{day}}{2722 \mathrm{~kg} \mathrm{U} / \text { day }}=8.8 \times 10^{-4} \frac{\mathrm{kg} \mathrm{Cr}}{\mathrm{kg} \mathrm{U}(\text { input })}
\end{aligned}
$$

\section{E.3.3.6 Molybdenum}

$$
\begin{aligned}
& (5, p .336) \\
& \left.\left(\frac{.3+16}{2}\right) \mathrm{mg} / \mathrm{l} \times 80 \mathrm{gal} / \mathrm{min} \times 1440 \mathrm{~min} / \text { day } \times 3.785 \mathrm{l} / \mathrm{ga}\right] \times \frac{\mathrm{kg}}{10^{6} \mathrm{mg}} \\
& =3.5 \mathrm{~kg} \mathrm{Mo} / \mathrm{day} \\
& \therefore \frac{3.5 \mathrm{~kg} \mathrm{Mo} / \text { day }}{2722 \mathrm{kgU} / \text { day }}=1.3 \times 10^{-3} \frac{\mathrm{kg} \mathrm{Mo}}{\mathrm{kgU} \text { (input) }}
\end{aligned}
$$

\section{B.3.3.9 Copper}

$$
\begin{aligned}
& (5, \mathrm{p} .337) \\
& \left(\frac{.7+3}{2}\right) \mathrm{mg} / 2 \times 80 \mathrm{gal} / \mathrm{min} \times 1440 \mathrm{~min} / \text { day } \times 3.785 \mathrm{l} / \mathrm{gal} \times \frac{\mathrm{kg}}{100 \mathrm{mg}} \\
& =.807 \mathrm{~kg} \mathrm{Cu} / \mathrm{day} \\
& \therefore \frac{.807 \mathrm{~kg} \mathrm{Cu} / \text { day }}{2722 \mathrm{~kg} \mathrm{U} / \text { day }}=3 \times 10^{-4} \frac{\mathrm{kg} \mathrm{Cu}}{\mathrm{kg} \mathrm{U} \text { (input) }}
\end{aligned}
$$

\section{B.3.3.8 Nickel}

(5, p. 337)

$1.4 \mathrm{mg} / 2 \times 80 \mathrm{gal} / \mathrm{min} \times 1440 \mathrm{~min} /$ day $\times 3.785 \mathrm{2} / \mathrm{gal} \times \frac{\mathrm{kg}}{100 \mathrm{mg}}=.610 \mathrm{~kg} \mathrm{Ni} /$ day

$\therefore \frac{.610 \mathrm{~kg} \mathrm{Ni} / \mathrm{day}}{2722 \mathrm{kgU} / \mathrm{day}}=2.2 \times 10^{-4} \frac{\mathrm{kg} \mathrm{Ni}}{\mathrm{kgU} \text { (input) }}$ 
8.3.3.9 Lead

$$
\begin{aligned}
& (5, \mathrm{p} .337) \\
& \left(\frac{.8+2}{2}\right) \mathrm{mg} / \mathrm{l} \times 80 \mathrm{gal} / \mathrm{min} \times 1440 \mathrm{~min} / \text { day } \times 3.785 \% / \mathrm{gal} \times \frac{\mathrm{kg}}{100 \mathrm{mg}} \\
& =.610 \mathrm{~kg} \mathrm{~Pb} / \mathrm{day} \\
& \therefore \frac{.670 \mathrm{~kg} \mathrm{~Pb} / \mathrm{day}}{2722 \mathrm{~kg} \mathrm{U} / \mathrm{day}}=2.2 \times 10^{-4} \frac{\mathrm{kg} \mathrm{Pb}}{\mathrm{kgU} \text { (input) }}
\end{aligned}
$$

\section{B.3.3.10 Zinc}

(5, p. 337)

$3 \mathrm{mg} / 2 \times 80 \mathrm{gal} / \mathrm{min} \times 1440 \mathrm{~min} /$ day $\times 3.785 \mathrm{l} / \mathrm{gal} \times \frac{\mathrm{kg}}{10^{0} \mathrm{mg}}=1.30 \mathrm{~kg} \mathrm{Zn} /$ day

$\therefore \frac{1.30 \mathrm{~kg} \mathrm{Zn} / \text { day }}{2722 \mathrm{~kg} \mathrm{U} / \text { day }}=4.8 \times 10^{-4} \frac{\mathrm{kg} \mathrm{Zn}}{\mathrm{kg} \mathrm{U} \text { (input) }}$

\section{B.3.3.11 Titanium}

(5, p. 336)

$7 \mathrm{mg} / 2 \times 80 \mathrm{gal} / \mathrm{min} \times 1440 \mathrm{~min} /$ day $\times 3.785 \mathrm{l} / \mathrm{gal} \times \frac{\mathrm{kg}}{100 \mathrm{mg}}=3.052 \mathrm{~kg} \mathrm{Ti} / \mathrm{day}$

$$
\therefore \frac{3.052 \mathrm{~kg} \mathrm{Ti} / \mathrm{day}}{2722 \mathrm{~kg} \mathrm{U} / \mathrm{day}}=1.12 \times 10^{-3} \frac{\mathrm{kg} \mathrm{Ti}}{\mathrm{kg} \mathrm{U}(\text { input })}
$$

\section{B.3.3.12 Sulfide}

$$
\begin{aligned}
& (5, \mathrm{p} .336) \\
& \left(\frac{0+.5}{2}\right) \mathrm{mg} / \mathrm{l} \times 80 \mathrm{gal} / \mathrm{min} \times 1440 \mathrm{~min} / \text { day } \times 3.785 \mathrm{l} / \mathrm{gal} \times \frac{\mathrm{kg}}{106 \mathrm{mg}} \\
& =.109 \mathrm{~kg} \mathrm{~S}^{-2} / \text { day } \\
& \therefore \frac{.109 \mathrm{~kg} \mathrm{~S}^{-2} / \text { day }}{2722 \mathrm{~kg} \mathrm{U} / \text { day }}=4 \times 10^{-5} \frac{\mathrm{kg} \mathrm{S}^{-2}}{\mathrm{~kg} \mathrm{U} \text { (input) }}
\end{aligned}
$$

\section{B.3.3.13 Cyanide}

$$
\text { (5, p. 336) }
$$




$$
\begin{aligned}
& \left(\frac{0+.01}{2}\right) \mathrm{rg} / \mathrm{x} \times 80 \mathrm{gal} / \mathrm{min} \times 1440 \mathrm{~min} / \mathrm{day} \times 3.785 \mathrm{z} / \mathrm{ga} \times \frac{\mathrm{kg}}{10^{0} \mathrm{mg}} \\
& =.002 \mathrm{~kg} \mathrm{Cn} / \mathrm{day} \\
& \therefore \frac{.002 \mathrm{~kg} \mathrm{Cn} / \mathrm{day}}{2722 \mathrm{~kg} \mathrm{U} / \mathrm{day}}=8 \times 10^{-7} \frac{\mathrm{kg} \mathrm{Cn}}{\mathrm{kgU} \text { (input) }}
\end{aligned}
$$

\section{B.3.3.14 Silver}

$$
\begin{aligned}
& (5, \mathrm{p} .337) \\
& \left(\frac{0+.01}{2}\right) \mathrm{mg} / 2 \times 80 \mathrm{gat} / \mathrm{min} \times 1440 \mathrm{~min} / \text { day } \times 3.785 \mathrm{2} / \mathrm{gal} \times \frac{\mathrm{kg}}{100 \mathrm{mg}} \\
& =.002 \mathrm{~kg} \mathrm{Ag} / \mathrm{day} \\
& \therefore \frac{.002 \mathrm{~kg} \mathrm{Ag} / \mathrm{day}}{2722 \mathrm{~kg} \mathrm{U} / \text { day }}=8 \times 10^{-7} \frac{\mathrm{kg} \mathrm{Ag}}{\mathrm{kg} \mathrm{U} \text { (input) }}
\end{aligned}
$$

\section{B.3.3.15 Manganese}

$$
\begin{aligned}
& (5, \mathrm{p} .337) \\
& \left(\frac{100+210}{2}\right) \mathrm{mg} / 2 \times 80 \mathrm{gal} / \mathrm{min} \times 1440 \mathrm{~min} / \text { day } \times 3.785 \mathrm{l} / \mathrm{gal} \times \frac{\mathrm{kg}}{100 \mathrm{mg}} \\
& =67.5 \mathrm{~kg} \mathrm{Mn} / \text { day } \\
& \therefore \frac{67.5 \mathrm{~kg} \mathrm{Hn} / \text { day }}{2722 \mathrm{~kg} \mathrm{~V} / \mathrm{day}}=.025 \frac{\mathrm{kg} / \mathrm{in}}{\mathrm{kgU} \text { (input) }}
\end{aligned}
$$

\section{B.3.3.16 Hagnesium}

$(5$, p. 336)

$550 \mathrm{mg} / 2 \times 80 \mathrm{gal} / \mathrm{min} \times 1440 \mathrm{~min} /$ day $\times 3.785 \mathrm{l} / \mathrm{gal} \times \frac{\mathrm{kg}}{10^{0} \mathrm{mg}}=240 \mathrm{~kg} \mathrm{Mg} /$ day

$\therefore \frac{240 \mathrm{~kg} \mathrm{Mg} / \mathrm{day}}{2722 \mathrm{~kg} \mathrm{U} / \mathrm{day}}=.088 \frac{\mathrm{kg} M \mathrm{Mg}}{\mathrm{kg} \mathrm{U} \text { (input) }}$

\subsubsection{Selenium}

According to Reference 6 , selenium is leached from ores in the alkaline circuit. Therefore, the release factor in an acid circuit is assumed zero. 


\section{B.3.3.18 Sulfuric Acid}

The pH of the tailings solution (9) provides a good estimate of the sulfuric acid content of the tailings pond.

$$
\begin{aligned}
& (9, \mathrm{p} .173) \\
& \mathrm{pH}=2--\log \left[\mathrm{H}^{+}\right] \\
& {[\mathrm{H+}]=10^{-2} \mathrm{M}}
\end{aligned}
$$

Since one mole of $\mathrm{H}_{2} \mathrm{SO}_{4}$ results in 2 moles of free $\mathrm{H}^{+}$ion, the concentration of $\mathrm{H}_{2} \mathrm{SO}_{4}$ is half the concentration of the $\mathrm{H}^{+}$ion:

$\frac{10^{-2} M}{2}$

$$
\mathrm{H}_{2} \mathrm{SO}_{3} / 2 \times .098 \mathrm{~kg} \mathrm{H} \mathrm{SO}_{4} / \mathrm{mole}^{2}=4.9 \times 10^{-4} \mathrm{~kg} \mathrm{H}_{2} \mathrm{SO}_{4} / 2
$$

$4.9 \times 10^{-4} \mathrm{~kg} \mathrm{H} \mathrm{SO}_{4} / 2 \times 80 \mathrm{gal} / \mathrm{min} \times 3.785 \mathrm{l} / \mathrm{gal} \times 1440 \mathrm{~min} . /$ day

$=214 \mathrm{~kg} \mathrm{H}_{2} \mathrm{SO}_{4} /$ day

\begin{tabular}{|c|c|c|c|c|c|c|}
\hline \multirow[b]{2}{*}{ Material Released } & \multicolumn{3}{|c|}{ Airborne } & \multicolumn{3}{|c|}{ Liquid } \\
\hline & Factor & Reliability & $\begin{array}{l}\text { Ref. } \\
\text { Calc. } \\
\end{array}$ & Factor & Reliability & $\begin{array}{l}\text { Ref. } \\
\text { Calc. } \\
\end{array}$ \\
\hline Chloride & & & & $3 \times 10^{-2}$ & 2 & 3.4 .3 .1 \\
\hline Aluminum & & & & $2 \times 10^{-4}$ & 2 & 3.4 .3 .2 \\
\hline Barium & & & & $7 \times 10^{-5}$ & 2 & B. 4.3 .3 \\
\hline Copper & & & & $5 \times 10^{-5}$ & 2 & B.4.3.4 \\
\hline Iron & & & & $5 \times 10^{-4}$ & 2 & B.4.3.5 \\
\hline Sodium & & & & $2 \times 10^{-2}$ & 2 & B.4.3.6 \\
\hline Zinc & & & & $2 \times 10^{-5}$ & 2 & B.4.3.7 \\
\hline Hydrogen Sulfide & 0 & 2 & & & & \\
\hline Armonia & & & & 0 & 2 & \\
\hline Nitric Acid & 0 & 2 & & 0 & 2 & \\
\hline
\end{tabular}

$$
\therefore \frac{214 \mathrm{~kg} \mathrm{H}_{2} \mathrm{jO}_{4} / \text { day }}{2722 \mathrm{~kg} \mathrm{U/day}}=.08 \frac{\mathrm{kg} \mathrm{H}_{2} \mathrm{SO}_{4}}{\mathrm{~kg} \mathrm{U}}
$$

TABLE B-4. Aqueous Converşion - Chemical Release Factor $\mathrm{kg} / \mathrm{kg} \mathrm{U}^{3}$ (input) 


\section{B.4 URANIUM PURIFICATION AND CONERSION:}

\subsubsection{ASSUMPTIONS}

The model plant produces the following two major liquid waste streams: 1) the solvent extraction circuit raffinate and 2) the waste hydrogen fluoride scrubber product. Only the latter fluoride stream is considered an effluent because part of it is discharged to the environment.

The fluoride stream consists of several smaller streams which combine prior to treatment. The resulting stream is then neutralized with slaked $1 \mathrm{ime}$ to a pH of 12 and routed to the fluoride sludge pond where the bulk of excess lime and calcium fluoride precipitate settle out. The overflow from the sludge pond is now treated with sulfuric acid to a pH of 7 , and the resulting solution is fed to a clarifying pond. Overflow from this clarifying pond is combined with "clean" waste water and sewage lagoon overflow and discharged from the facility to the receiving river at a rate of $2500 \mathrm{gal} / \mathrm{min} .(13, \mathrm{p.13})$ Liquid effluents are calculated assuming the analyses of these discharge streams at the Kerr-McGee, Sequoyah facility are representative of the ENFORM model plant.

The uranium input to this facility, calculated in Appendix $A,(1$, calc. 4-1) is assumed to be $2.485 \times 10^{4} \mathrm{~kg} \mathrm{U} /$ day.

\section{B.4.2 AIRBORNE RELEASES}

Nitric Acid

Process off gases are treated to recover gaseous nitric acid which is then returned for reuse to the process. $(14, p$. III-7) Any 1 iquid nitric acid that is released is neutralized and impounded in storage ponds. (12, p. III-9) Hydrogen Sulfide

Hydrogen sulfide, released from the reduction tower, is burned in air to water vapor and sulfur oxide and discharged to the plant stack. Theoretically, then, no hydrogen sulfide is released to the atmosphere. (12, p. III-7)

Ammonia

Anmonia released from the conversion process is also retained in storage ponds. $(15, \mathrm{p} .27)$ 
8. 4.3 LIQUID RELEASES

B.4.3.1 Chtoride

(14, p. v-5)

$50 \mathrm{mg} / \mathrm{i} \times 2500 \mathrm{gal} / \mathrm{min} \times 3.785 \mathrm{\ell} / \mathrm{gai} \times 1440 \mathrm{~min} /$ day $\times \frac{\mathrm{kg}}{10^{6} \mathrm{mg}}$

$=681.3 \mathrm{~kg} \mathrm{Chloride/day}$

$\therefore \frac{681.3 \mathrm{~kg} \mathrm{ChToride} / \text { day }}{2.485 \times 10^{4} \mathrm{~kg} \mathrm{U} / \mathrm{day}}=.027 \frac{\mathrm{kg} \mathrm{Ch} \text { loride }}{\mathrm{kg} \mathrm{U}}$

B.4.3.2 Aluminum

$(74, p \cdot V-5)$

$.40 \mathrm{mg} / 2 \times 2500 \mathrm{gal} / \mathrm{min} \times 3.785 \mathrm{l} / \mathrm{gal} \times 1440 \mathrm{~min} / \mathrm{day} \times \frac{\mathrm{kg}}{10^{6} \mathrm{mg}}$

$=5.45 \mathrm{~kg} \mathrm{Al} / \mathrm{day}$

$\therefore \frac{5.45 \mathrm{~kg} \mathrm{Al} / \mathrm{day}}{2.485 \times 10^{4} \mathrm{~kg} \mathrm{U} / \mathrm{day}}=2.2 \times 10^{-4} \frac{\mathrm{kg} \mathrm{A}}{\mathrm{kg} \mathrm{U}}$

B. 4.3.3 Barium

(14, p. $V-5)$

$.125 \mathrm{mg} / \mathrm{l} \times 2500 \mathrm{gal} / \mathrm{min} \times 3.785 \mathrm{\ell} / \mathrm{gal} \times 1440 \mathrm{~min} /$ day $\times \frac{\mathrm{kg}}{10^{6} \mathrm{mg}}$

$=1.7 \mathrm{~kg} \mathrm{Ba} / \mathrm{day}$

$\therefore \frac{1.7 \mathrm{~kg} \mathrm{Ba} / \text { day }}{2.485 \times 10^{4} \mathrm{~kg} \mathrm{U} / \text { day }}=5.85 \times 10^{-5} \frac{\mathrm{kg} \mathrm{Ba}}{\mathrm{kg} \mathrm{U}}$

B. 4.3.4 Copper

$(14, p \cdot V-5)$

$.09 \mathrm{mg} / 2 \times 2500 \mathrm{gal} / \mathrm{min} \times 3.785 \mathrm{2} / \mathrm{gal} \times 1440 \mathrm{~min} / \mathrm{day} \times \frac{\mathrm{kg}}{10^{6} \mathrm{mg}}$

$=1.2 \mathrm{~kg} \mathrm{Cu} /$ day

$\therefore \frac{1.2 \mathrm{~kg} \mathrm{Cu} / \mathrm{day}}{2.485 \times 10^{4} \mathrm{~kg} \mathrm{U} / \mathrm{day}}=4.9 \times 10^{-5} \frac{\mathrm{kg} \mathrm{Cu}}{\mathrm{kg} \mathrm{U}}$ 
3.4 .3 .5 Iron

(14, p. V-5)

$1 \mathrm{mg} / 2 \times 2500 \mathrm{gal} / \mathrm{min} \times 3.785 \mathrm{l} / \mathrm{ga}] \times 1440 \mathrm{~min} / \mathrm{day} \times \frac{\mathrm{kg}}{10^{\sigma} \mathrm{mg}}=13.6 \mathrm{~kg} \mathrm{Fe} /$ day

$\therefore \frac{13.5 \mathrm{~kg} F \mathrm{Fe} / \text { day }}{2.485 \times 10^{4} \mathrm{~kg} \mathrm{U} / \mathrm{day}}=5.5 \times 10^{-4} \frac{\mathrm{kg} \mathrm{Fe}}{-\mathrm{kg} \mathrm{U}}$

B.4.3.5 Sodium

(14, p. $V-5)$

$35 \mathrm{mg} / 2 \times 2500 \mathrm{gal} / \mathrm{min} \times 3.785 \mathrm{2} / \mathrm{gal} \times 1440 \mathrm{~min} /$ day $\times \frac{\mathrm{kg}}{10^{6} \mathrm{mg}}=476.9 \mathrm{Na} /$ day

$\therefore \frac{476.9 \mathrm{~kg}, \mathrm{Na} / \mathrm{day}}{2.485 \times 10^{4} \mathrm{~kg} \mathrm{U} / \mathrm{day}}=.019 \frac{\mathrm{kg} \mathrm{Na}}{\mathrm{kg} \mathrm{U}}$

B. 4.3.7 Zinc

(14, p. $V-5)$

$.04 \mathrm{mg} / 2 \times 2500 \mathrm{ga} / \mathrm{min} \times 3.785 \mathrm{l} / \mathrm{ga} 1 \times 1440 \mathrm{~min} /$ day $\times \frac{\mathrm{kg}}{10^{0} \mathrm{mg}}=.55 \mathrm{~kg} \mathrm{Zn} /$ day

$\therefore \frac{.55 \mathrm{~kg} \mathrm{Zn} / \text { day }}{2.485 \times 10^{4} \mathrm{~kg} \mathrm{U} / \text { day }}=2.2 \times 10^{-5} \frac{\mathrm{kg} \mathrm{Zn}}{--\mathrm{kg} \mathrm{U}}$ 
TABLE B-5. Gaseous Diffusion Plant Chemical Release

Factors $\mathrm{kg} / \mathrm{kg} U$ (produced)

\begin{tabular}{|c|c|c|c|c|c|c|}
\hline \multirow[b]{2}{*}{ Material Released } & \multicolumn{3}{|c|}{ Airborne } & \multicolumn{3}{|c|}{ Liauid } \\
\hline & Factor & Reliability & $\begin{array}{l}\text { Ref. } \\
\text { calc. }\end{array}$ & Factor & Reliability & $\begin{array}{l}\text { Ref. } \\
\text { Caic. }\end{array}$ \\
\hline Nitirc Acid & $?$ & & & & & \\
\hline Boric Acid & $?$ & & & & & \\
\hline Nickel sulfate & $?$ & & & & & \\
\hline Nickel Chloride & ? & & & & & \\
\hline Hydrogen Fluoride & $1 \times 10^{-3}$ & 2 & 5.2 .1 & & & \\
\hline Armonia & & & & $3 \times 10^{-3}$ & 2 & 5.3 .1 \\
\hline Cadmium & & & & $1 \times 10^{-5}$ & 2 & 5.3 .2 \\
\hline Calcium & & & & $9 \times 10^{-2}$ & 2 & 5.3 .3 \\
\hline Chioride & & & & $1 \times 10^{-1}$ & 2 & 5.3 .4 \\
\hline Copper & & & & $2 \times 10^{-4}$ & 2 & 5.3 .5 \\
\hline Iron & & & & $6 \times 10^{-3}$ & 2 & 5.3 .6 \\
\hline Nicke 1 & & & & $6 \times 10^{-4}$ & 2 & 5.3 .7 \\
\hline Sodium & & & & $1 \times 10^{-1}$ & 2 & 5.3 .8 \\
\hline Sulfate & & & & $9 \times 10^{-2}$ & 2 & 5.3 .9 \\
\hline Sulfites & & & & $1 \times 10^{-4}$ & 1 & 5.3 .10 \\
\hline Boron & & & & $1 \times 10^{-4}$ & 1 & 5.3 .10 \\
\hline Magnesium & & & & $1 \times 10^{-4}$ & 1 & 5.3 .10 \\
\hline Molybdenum & & & & $1 \times 10^{-4}$ & 1 & 5.3 .10 \\
\hline Silver & & & & $1 \times 10^{-4}$ & 1 & 5.3 .10 \\
\hline Manganese & & & & $1 \times 10^{-4}$ & 1 & 5.3 .10 \\
\hline
\end{tabular}




\subsection{GASEOUS DIFFUSION ENRICHMENT}

\section{B.5.? ASSLMPTIONS}

The following values are assumed from calculations by Tom Powers.

$\begin{array}{cc}\text { Value } & \frac{\text { Calculation }}{5-1} \\ 5708 \mathrm{~kg} \mathrm{U} / \text { product/day } & 5-18\end{array}$

The concentrations in the effluent stream utilized for liquid release calculations are derived from the Oak Ridge GDP. However, the values are also assumed to be representative of the Portsmouth GDP $(16, p . D-12)$ and therefore the ENFORM model GDP.

\section{B.5.2 AIRBORNE RELEASES}

B.5.2.1 Hydrogen Fluoride (gas)

(17, p. 2.3-145)

$2.4 \mathrm{MT} / \mathrm{yr} \times 1000 \mathrm{~kg} / \mathrm{MT} \times 1 \mathrm{yr} / 365$ day $=6.6 \mathrm{~kg} / \mathrm{day}$

$\therefore \frac{6.6 \mathrm{~kg} / \mathrm{day}}{5708 \mathrm{~kg} \mathrm{U} / \mathrm{day}}=1.2 \times 10^{-3} \frac{\mathrm{kg} \mathrm{HF}}{\mathrm{kg} \mathrm{U}}$

Airborne effluents include nitric acid, boric acid, nickel sulfate, and nickel chloride. Due to insufficient data, no release factors are calculated for these.

\section{B.5.3 LIQUID RELEASES}

\section{B.5.3.1 Ammonia}

(16, p. D-19)

$1 \mathrm{mg} / \ell \mathrm{NH}_{4}^{+} \times 1.71 \times 10^{7} \ell /$ day $\times \frac{1}{10^{-6} \mathrm{mg} / \mathrm{kg}}=17.1 \mathrm{~kg} \mathrm{NH}_{4}^{+} /$day 


$$
\therefore \frac{17.7 \mathrm{~kg} \mathrm{HH} z / \mathrm{day}}{3708 \mathrm{~kg} \mathrm{U} / \mathrm{day}}=3.00 \times 10^{-3} \frac{\mathrm{kg} \mathrm{NH} 3}{\mathrm{~kg} \mathrm{(product)}}
$$

\section{B.5.3.2 Cadmium}

$$
\begin{aligned}
& (16, \mathrm{p} . \mathrm{D}-19) \\
& .004 \mathrm{mg} / 2 \times 1.71 \times 10^{7} 2 / \text { day } \times \frac{\mathrm{kg}}{106 \mathrm{mg}}=.0684 \mathrm{~kg} \mathrm{Cd} / \text { day } \\
& \therefore \frac{.0684 \mathrm{~kg} \mathrm{Cd} / \text { day }}{5708 \mathrm{kgU} / \text { day }}=1.20 \times 10^{-5} \frac{\mathrm{kg} \mathrm{Cd}}{\mathrm{kgU} \text { (product) }}
\end{aligned}
$$

\section{B.5.3.3 Calcium}

(16, p. D-19)

$30 \mathrm{mg} / 2 \times 1.71 \times 10^{7}$ e/day $\times \frac{\mathrm{kg}}{10^{6} \mathrm{mg}}=513 \mathrm{~kg} \mathrm{Ca} /$ day

$$
\therefore \frac{513 \mathrm{~kg} \mathrm{Ca} / \text { day }}{5708 \mathrm{~kg} \mathrm{U} / \text { day }}=9 \times 10^{-2} \frac{\mathrm{kg} \mathrm{Ca}}{\mathrm{kg} \mathrm{U} \text { (product) }}
$$

\section{B.5.3.4 Chloride}

(16, p. D-19)

$45 \mathrm{mg} / \mathrm{\ell} \times 1.71 \times 10^{7}$ थ/day $\times \frac{\mathrm{kg}}{10^{6} \mathrm{mg}}=7.69 .5 \mathrm{~kg}$ Chioride $/$ day

$\therefore \frac{769.5 \mathrm{~kg} \mathrm{Chloride} / \text { day }}{5708 \mathrm{~kg} \mathrm{U} / \mathrm{day}}=.13 \frac{\mathrm{kg} \text { Chloride }}{\mathrm{kg} \mathrm{U}}$

\section{B.5.3.5 Copper}

(16, 0-19)

$.07 \mathrm{mg} / \mathrm{l} \times 1.71 \times 10^{7} \mathrm{\ell} /$ day $\times \frac{\mathrm{kg}}{10^{6} \mathrm{mg}}=1.2 \mathrm{~kg} \mathrm{Cu} /$ day

$\therefore \frac{1.2 \mathrm{~kg} \mathrm{Cu}}{5708 \mathrm{~kg} \mathrm{U}}=2.1 \times 10^{-4} \frac{\mathrm{kg} \mathrm{Cu}}{\mathrm{kg} \mathrm{U}}$

\section{B.5.3.6 Iron}

(16, p. D-19)

$2 \mathrm{mg} \mathrm{Fe} / \mathrm{\ell} \times 1.71 \times 10^{7} \mathrm{\ell} /$ day $\times \frac{\mathrm{kg}}{10^{6} \mathrm{mg}}=34.2 \mathrm{~kg} \mathrm{Fe} /$ day 


$$
\therefore \frac{34.2 \mathrm{~kg} \mathrm{Fe} / \mathrm{day}}{5708 \mathrm{~kg} \mathrm{U} / \mathrm{day}}=6.0 \times 10^{-3} \frac{\mathrm{kg} \mathrm{Fe}}{\mathrm{kg} \mathrm{U}}
$$

\section{B.5.3.7 Nicke]}

$(16, p .0-19)$

$.2 \mathrm{mg} / 2 \times 1.71 \times 10^{7}$ l/day $\times \frac{\mathrm{kg}}{10^{6} \mathrm{mg}}=3.42 \mathrm{~kg} \mathrm{Ni} /$ day

$\therefore \frac{3.42 \mathrm{~kg} \mathrm{Ni} / \mathrm{day}}{5708 \mathrm{~kg} \mathrm{U} / \mathrm{day}}=6.0 \times 10^{-4} \frac{\mathrm{kg} \mathrm{Ni}}{\mathrm{kg} \mathrm{U}}$

\section{B.5.3.8 Sodium}

$$
\text { (16, p. D-19) }
$$

$45 \mathrm{mg} / \imath \times 1.71 \times 10^{7} \ell /$ day $\times \frac{\mathrm{kg}}{10^{6} \mathrm{mg}}=769.5 \mathrm{~kg} \mathrm{Na} /$ day

$\therefore \frac{769.5 \mathrm{~kg} \mathrm{Na} / \mathrm{day}}{5708 \mathrm{~kg} \mathrm{U} / \mathrm{day}}=.13 \frac{\mathrm{kg} \mathrm{Na}}{\mathrm{kg} \mathrm{U}}$

\section{B.5.3.9 Sulfate}

Same as calculation B.5.3.3 for calcium.

\section{B.5.3.10 Other Liquid Effluents}

No data has been found to determine release factors for the remaining liquid effluents. Assuming the constituents all result from impurities in the uranium ore, they will be present in the enrichment tailings in low concentration. Therefore, a concentration of .05 ppm shall be assumed, resulting in the following release factor for each of the following: sulfites, boron, magnesium, molybdenum, silver, and manganese.

$$
\begin{aligned}
& .05 \mathrm{mg} / \ell \times 1.71 \times 10^{7} \text { \&/day } \times \frac{\mathrm{kg}}{10^{6} \mathrm{mg}}=.855 \mathrm{~kg} / \text { day } \\
& \therefore \frac{.855 \mathrm{~kg} / \text { day }}{5708 \mathrm{~kg} \mathrm{U} / \mathrm{day}}=1.5 \times 10^{-4} \frac{\mathrm{kg}}{\mathrm{kgU}}
\end{aligned}
$$


IABLE B-6. Fuel Fabrication Cherical Release

Factors kg/kg U (input)

\begin{tabular}{|c|c|c|c|c|c|c|}
\hline \multirow[b]{2}{*}{ Material Released } & \multicolumn{3}{|c|}{ Airborne } & \multicolumn{3}{|c|}{ Liguid } \\
\hline & Factor & Reliability & $\begin{array}{l}\text { Refi } \\
\text { Caic. }\end{array}$ & Factor & Reliability & $\begin{array}{l}\text { Ref. } \\
\text { Calc. }\end{array}$ \\
\hline \multicolumn{7}{|l|}{ Chromium } \\
\hline Zirconium & $5 \times 10^{-3}$ & 1 & 6.2 .1 & & & \\
\hline Nitric Acid & $5 \times 10^{-3}$ & 1 & 6.2 .2 & & & \\
\hline$x y 101$ & $1 \times 10^{-3}$ & 1 & 6.2 .3 & & & \\
\hline Butanol & $1 \times 10^{-3}$ & 1 & 6.2 .4 & & & \\
\hline $\begin{array}{l}\text { Methyl Butyl } \\
\text { Ketone }\end{array}$ & $9 \times 10^{-4}$ & 1 & 6.2 .5 & & & \\
\hline Glycol Ethyl Ether & $7 \times 10^{-4}$ & 1 & 6.2 .6 & & & \\
\hline Isopropyl Alcohol & $5 \times 10^{-3}$ & 1 & 6.2 .7 & & & \\
\hline Nickel Sulfate & $?$ & & & & & \\
\hline Nickel Chloride & $?$ & & & & & \\
\hline Sulfuric Acid & $?$ & & & & & \\
\hline Boric Acid & $?$ & & & & & \\
\hline Ace tone & ? & & & & & \\
\hline Zinc & $?$ & & & & & \\
\hline Copper & & & & $1 \times 10^{-5}$ & 1 & 6.3 .1 \\
\hline Nitrate & & & & $8 \times 10^{-3}$ & 2 & 6.3 .2 \\
\hline
\end{tabular}




\section{B.6. FUEL FABRICATION}

\section{B.6.1 ASSLMPTIONIS}

The following value was assumed in Appendix A:

$$
\begin{aligned}
& 4404 \mathrm{~kg} \mathrm{U}(\text { input - enriched to } 3.2 \% \text { )/day } \\
& \text { (the plant output is } 1600 \mathrm{MTM} / \text { year or } 4.38 \mathrm{MTM} / \text { day) }
\end{aligned}
$$

The chemical effluent information for calculations 6.2 .1 to 6.2 .7 and 6.3.1 was reported for the General Electric Fuel Fabrication facility. (19) It is assumed to be representative of the ENFORM model. Reference 19 lists effluent quantities for the cladding plant and two supporting facilities. The calculations below are adjusted for just the cladding plant.

\section{B.6.2 AIRBORNE RELEASES}

\section{B.6.2.1 Zirconium (air)}

(Appendix C, Table $\mathrm{C}-1$ )

$\frac{5.43 \mathrm{~kg} \mathrm{Zr}}{\text { MTU }} \times \frac{M T}{1000 \mathrm{~kg}}=5.43 \times 10^{-3} \frac{\mathrm{kg} \mathrm{Zr}}{\mathrm{kg} \mathrm{U}}$

B.6.2.2 Nitric Acid (air)

(Appendix C, Table $\mathrm{C}-1$ )

$\frac{5.48 \mathrm{~kg} \mathrm{HNO}}{3 T U} \times \frac{\mathrm{MT}}{1000 \mathrm{~kg}}=5.48 \times 10^{-3} \frac{\mathrm{kg} \mathrm{HNO}}{3 \mathrm{~kg} \mathrm{U}}$

\subsection{3 xy101 (air)}

(Appendix C, Table C-1)

$3.83 \mathrm{~kg} /$ MTU $\times \frac{\mathrm{MT}}{1000 \mathrm{~kg}} \times \frac{1}{3}^{(\mathrm{a})}=1.28 \times 10^{-3} \frac{\mathrm{kg} \times \mathrm{Xylol}}{\mathrm{kg} \mathrm{U}}$

\section{B.6.2.4 Butanol (air)}

(Appendix C, Table C-1)

$4.38 \mathrm{~kg} /$ MTU $\times \frac{\text { MT }}{1000 \mathrm{~kg}} \times \frac{1}{3}^{(\mathrm{a})}=1.46 \times 10^{-3} \frac{\mathrm{kg} \mathrm{Butanol}}{\mathrm{Kg} \mathrm{U}}$

(a) Assumed portion for cladding plant 


\subsubsection{Methy? Buty! Ketore (air)}

(Ácpendix $C$, Tadie $(-T)$

$2.74 \mathrm{~kg} / \mathrm{MTU} \times \frac{\mathrm{MT}}{1000 \mathrm{~kg}} \times \frac{7}{3}^{(a)}=9.13 \times 10^{-4} \frac{\mathrm{kg} \text { Vethyl Butyl Ketore }}{\mathrm{kg} \mathrm{U}}$

B.6.2.6 Glycol Ethy? Ether (air)

(Appendix C, Table $\mathrm{C}-1$ )

$2.19 \mathrm{~kg} / \mathrm{MTU} \times \frac{\mathrm{MT}}{1000 \mathrm{~kg}} \times \frac{i}{3}^{(\mathrm{a})}=7.30 \times 10^{-4} \frac{\mathrm{kg} \mathrm{Glycol} \mathrm{Ethyl} \mathrm{Ether}}{\mathrm{kg} \mathrm{U}}$

B.6.2.7 Isopropyl Alcohol (air)

(Appendix C, Table C-1)

$13.7 \mathrm{~kg} / \mathrm{MTU} \times \frac{\mathrm{MT}}{1000 \mathrm{~kg}} \times \frac{1}{3}^{(\mathrm{a})}=4.57 \times 10^{-3} \frac{\mathrm{kg} \text { [sopropy] Alcohol }}{\mathrm{kg} \mathrm{U}}$

B.6.2.8 No release factors were computed for the following, as data were not available.

Nickel sulfate (air)

Nickel chloride (air)

Sulfuric acid (air)

Boric acid (air)

Acetone

Zinc

\section{B.6.3 LIQUID RELEASES}

B.6.3.1 Copper

(Appendix C, Table C-2)

$.011 \mathrm{~kg} /$ MTU $\times \frac{M T}{1000 \mathrm{~kg}}=1.1 \times 10^{-5} \frac{\mathrm{kg} \mathrm{Cu}}{\mathrm{Kg} \mathrm{U}}$ 
B.6.3.2 Nitrates

$$
\begin{aligned}
& (20, \% .9) \\
& 81 \mathrm{ib} \mathrm{NO}_{3} / \text { day } x .4536 \mathrm{~kg} / 1 \mathrm{~b}=36.7 \mathrm{~kg} \mathrm{NO} / \mathrm{day} \\
& \therefore \frac{36.7 \mathrm{~kg} \mathrm{NO} / \mathrm{day}}{4404 \mathrm{~kg} \mathrm{U} / \mathrm{day}}=8.3 \times 10^{-3} \frac{\mathrm{kg} \mathrm{NO}}{\mathrm{kg} \mathrm{U}} 3
\end{aligned}
$$

TABLE B-7. Pressurized Water Reactor Chemical

\begin{tabular}{|c|c|c|c|c|c|c|}
\hline \multirow[b]{2}{*}{ Material Released } & \multicolumn{3}{|c|}{ Airborne } & \multicolumn{3}{|c|}{ Liquid } \\
\hline & Factor & Reliability & $\begin{array}{r}\text { Ref. } \\
\text { Calc. } \\
\end{array}$ & Factor & Reliability & $\begin{array}{r}\text { Ref. } \\
\text { Calc. }\end{array}$ \\
\hline Chlorine & & & & $5 \times 10^{-3}$ & 2 & 7.3 .1 \\
\hline Phosphates & & & & $2 \times 10^{-2}$ & 2 & 7.3 .2 \\
\hline Lithium & & & & $4 \times 10^{-3}$ & 2 & 7.3 .3 \\
\hline Sulfur Oxides & $3 \times 10^{-3}$ & 1 & 7.2 .11 & & & \\
\hline Nitrogen 0xides & $8 \times 10^{-3}$ & 1 & 7.2 .14 & & & \\
\hline Particulates & $1 \times 10^{-3}$ & 1 & 7.2 .10 & & & \\
\hline Carbon Monoxide & $4 \times 10^{-4}$ & 1 & 7.2 .12 & & & \\
\hline Al dehydes & $2 \times 10^{-4}$ & 1 & 7.2 .15 & & & \\
\hline Hydrocarbons & $3 \times 10^{-4}$ & 1 & 7.2 .13 & & & \\
\hline Sulfuric Acid & $?$ & & & & $=$ & \\
\hline Calcium & 1 & 2 & 7.2 .1 & & & \\
\hline Magnesium & $1 \times 10^{-1}$ & 2 & 7.2 .2 & & & \\
\hline Sodium & $2 \times 10^{-1}$ & 2 & 7.2 .3 & & & \\
\hline Chloride & $2 \times 10^{-1}$ & 2 & 7.2 .4 & & & \\
\hline Sulfate & 2 & 2 & 7.2 .5 & & & \\
\hline Bicarbonate & $8 \times 10^{-2}$ & 2 & 7.2 .6 & & & \\
\hline Silica & $3 \times 10^{-1}$ & 2 & 7.2 .7 & & & \\
\hline \multirow[t]{2}{*}{ Potassium } & $2 \times 10^{-2}$ & 2 & 7.2 .8 & & & \\
\hline & Radi & uclide Releas & ctor, $\mathrm{kg}$ & eased/kg & ionuclide : in & \\
\hline Carbon 14 & 0.30 & 2 & 7.4 & & & \\
\hline
\end{tabular}
Release Factors $\mathrm{kg} / \mathrm{kg} \cup$ (input

Chemical Release Factors $\mathrm{kg} / \mathrm{kg} U$ (input) 



\subsection{PRESSURIZED WATER REACTOR}

\section{B.7 ASSUMPTIONS}

The capacity of the model piant, $102 \mathrm{~kg} \mathrm{U}$ (input) per day, was previousiy calculated in Appendix $A^{(1)}$ and is used extensively for PWR release factor calculations.

Waste heat from the Trojan plant is dissipated to the atmosphere by means of a single large natural draft cooling tower. A small portion of the circulating water will be lost from the cooling tower in the form of small droplets or drift. Average drift for a natural draft cooling tower is approximately $116 \mathrm{gpm}$. However, Trojan utilizes a drift eliminator which reduces cooling tower drift to about $43 \mathrm{gpm}$. $(21, \mathrm{p}$. III-5) The $43 \mathrm{gpm}$ drift is of the same composition as the circulating water containing $\simeq 1800 \mathrm{ppm}$ dissolved solids. (21, p. III-15) This drift is assumed an airborne effluent. (See calculations 7.2 .1 to 7.2 .8$.

\section{B.7.2 AIRBORNE EFFLUENTS}

\section{B.7.2.1 Calcium}

$(21, p$. II I-48)

$600 \mathrm{mg} / \mathrm{\ell} \times 43 \mathrm{gal} / \mathrm{min} \times 3.785 \mathrm{\ell} / \mathrm{gal} \times 1440 \mathrm{~min} /$ day $\times \frac{\mathrm{kg}}{10^{6} \mathrm{mg}}$

$=140.6 \mathrm{~kg} \mathrm{Ca} / \mathrm{day}$

$\therefore \frac{140.6 \mathrm{~kg} \mathrm{Ca} / \mathrm{day}}{102 \mathrm{~kg} \mathrm{U} / \mathrm{day}}=1.38 \frac{\mathrm{kg} \mathrm{Ca}}{\mathrm{kg} \mathrm{U}(\text { input) }}$

\section{B.7.2.2 Magnesium}

(21, p. II I-48)

$60 \mathrm{mg} / \mathrm{l} \times 43 \mathrm{gal} / \mathrm{min} \times 3.785 \mathrm{l} / \mathrm{ga} 1 \times 1440 \mathrm{~min} /$ day $\times \frac{\mathrm{kg}}{10^{5} \mathrm{mg}}$

$=14.06 \mathrm{~kg} \mathrm{Mg} / \mathrm{day}$

$\therefore \frac{14.06 \mathrm{~kg} \mathrm{Mg} / \mathrm{day}}{102 \mathrm{~kg} \mathrm{U} / \mathrm{day}}=.138 \frac{\mathrm{kg} \mathrm{Mg}}{\mathrm{kgU} \text { (input) }}$ 


\section{$\$ 7.2 .3$ Sodium}

(21, P. III-43)

$75 \mathrm{mg} / 2 \times 43 \mathrm{gal} / \mathrm{min} \times 1440 \mathrm{~min} / \mathrm{day} \times 3.7852 / \mathrm{gal} \times \frac{\mathrm{kg}}{100 \mathrm{mg}}=17.6 \mathrm{~kg} \mathrm{Na} / \mathrm{day}$

$\therefore \frac{17.8 \mathrm{~kg} \mathrm{Na} / \mathrm{day}}{102 \mathrm{~kg} \mathrm{U} / \mathrm{day}}=.172 \frac{\mathrm{kg} \mathrm{Na}}{\mathrm{kg} \mathrm{U}}$

3.7.2.4 Chloride

(21, P. III-48)

$70 \mathrm{mg} / 2 \times 43 \mathrm{gal} / \mathrm{min} \times 1440 \mathrm{~min} /$ day $\times 3.785 \mathrm{l} / \mathrm{ga} 3 \times \frac{\mathrm{kg}}{106 \mathrm{mg}}=16.4 \mathrm{~kg}$

$\therefore \frac{16.4 \mathrm{~kg} \mathrm{Cl} / \mathrm{day}}{102 \mathrm{~kg} \mathrm{U} / \mathrm{day}}=.161 \frac{\mathrm{kg} \mathrm{Cl}}{\mathrm{kg} \mathrm{U}}$

B.7.2.5 Sulfate

(21, p. III-48)

$800 \mathrm{mg} / 2 \times 43 \mathrm{gal} / \mathrm{min} \times 1440 \mathrm{~min} /$ day $\times 3.785 \mathrm{\ell} / \mathrm{gal} \times \frac{\mathrm{kg}}{10^{6} \mathrm{mg}}=187 \mathrm{~kg} \mathrm{SO} /$ day

$\therefore \frac{187 \mathrm{~kg} \mathrm{SO}}{102 \mathrm{~kg} \mathrm{U}} / \frac{\mathrm{day}}{\mathrm{day}}=1.8 \frac{\mathrm{kg} \mathrm{SO}}{\mathrm{kg} \mathrm{U}} 4$

\section{B.7.2.6 Bicarbonate}

(21, p. III-48)

$35 \mathrm{mg} / 2 \times 43 \mathrm{gal} / \mathrm{min} \times 1440 \mathrm{~min} /$ day $\times 3.785 \mathrm{l} / \mathrm{gal} \times \frac{\mathrm{kg}}{10^{6} \mathrm{mg}}=8.2 \mathrm{~kg} \mathrm{HCO} /$ day

$\therefore \frac{8.2 \mathrm{~kg} \mathrm{HCO}}{102 \mathrm{~kg} \mathrm{~L} / \mathrm{day}} \frac{\mathrm{day}}{\mathrm{day}}=.08 \frac{\mathrm{kg} \mathrm{HCO}}{\mathrm{kg} \mathrm{U}} 3$

\section{B.7.2.7 silica}

(21, p. III-48)

$150 \mathrm{mg} / 3 \times 43 \mathrm{gal} / \mathrm{min} \times 1440 \mathrm{~min} / \mathrm{day} \times 3.785 \mathrm{2} / \mathrm{gal} \times \frac{\mathrm{kg}}{10^{6} \mathrm{mg}}$

$=35.16 \mathrm{~kg} \mathrm{silica} /$ day 


$$
\therefore \frac{35.16 \mathrm{~kg} \mathrm{silica/ciay}}{102 \mathrm{~kg} \mathrm{U} / \mathrm{day}}=.345 \frac{\mathrm{kg} \mathrm{silica}}{\mathrm{kg} \mathrm{L}}
$$

\section{B.7.2.8 Potassium}

(2), p. III-43)

$10 \mathrm{mg} / 2 \times 43 \mathrm{gal} / \mathrm{min} \times 1440 \mathrm{~min} / \mathrm{day} \times 3.785 \mathrm{e} / \mathrm{ga}] \times \frac{\mathrm{kg}}{105 \mathrm{mg}}=2.3 \mathrm{~kg} \mathrm{~K} / \mathrm{day}$

$\therefore \frac{2.3 \mathrm{~kg} \mathrm{k} / \mathrm{day}}{102 \mathrm{~kg} \mathrm{U} / \mathrm{day}}=.023 \frac{\mathrm{kg} \mathrm{K}}{\mathrm{kg} \mathrm{U}}$

\section{B.7.2.9 Combustion Products}

As a source of auxiliary electric power required for emergency power generation, two diesel electric sets are available on site. Each set is operated at least once a month for one hour as a part of regular maintenance. These sets would be activated for power generation in the unlikely event that power is not available from transmission lines. These diesels burn about 4.5 gpm of No. 2 fuel oil per engine. (22, $0.3 .8-1)$

$(22, p, 3.8-1)$

$\frac{4.5 \mathrm{gal} \text { fuel oil/min }}{\text { engine }} \times 2$ diesel engines $\times 60 \mathrm{~min} / \mathrm{hr} \times \frac{1 \mathrm{hr}}{\mathrm{mo}} \times 3.785 \mathrm{2} / \mathrm{gal}$

$=2044 \mathrm{l} / \mathrm{mo}$ No. 2 fuel oil

To keep the calculations in terms consistent with the use (monthly rather than daily) of the diesel sets, the uranium input shal1 be converted to a monthly basis aiso.

$102 \mathrm{~kg} \mathrm{U} /$ day $\times 290$ operating days $/ \mathrm{yr}^{(1)} \times 1 \mathrm{yr} / 12 \mathrm{mo}=2465 \mathrm{~kg} \mathrm{~J} / \mathrm{mo}$

This will be used for the following calculations.

B.7.2.10 Particulates

$$
(23, p, 1.3-2)
$$

2044 \& fuel $/ \mathrm{mo} \times 1.8 \mathrm{~kg} / 1000 \ell=3.68 \mathrm{~kg} / \mathrm{mo}$

$$
\therefore \frac{3.63 \mathrm{~kg} / \mathrm{mo}}{2465 \mathrm{~kg} \mathrm{U} / \mathrm{mo}}=1.5 \times 10^{-3} \frac{\mathrm{kg} \text { Particulates }}{\mathrm{kg} \mathrm{U}}
$$


B. 7.2.11 Sulfur Oxides

$$
(s=22 \% \text { sulfur by weight })^{(24)}
$$

$$
\begin{aligned}
& 2044 \text { \& fue } 1 / \mathrm{mo} \times \frac{23, \mathrm{p} \cdot 1.3-2}{10002} \times \frac{17.25 \mathrm{~kg} \mathrm{so}}{1000} \times .22=7.76 \mathrm{~kg} \mathrm{so} x_{x} / \mathrm{mo} \\
& \therefore \frac{7.76 \mathrm{~kg} \mathrm{SO}}{2465 \mathrm{~kg} U} / \frac{\mathrm{mo}}{\mathrm{mo}}=3 \times 10^{-3} \frac{\mathrm{kg} \mathrm{SO}}{\mathrm{kg} \mathrm{U}} \times
\end{aligned}
$$

\section{B. 7.2.12 Carbon Monoxide}

$$
\begin{aligned}
& \left.2044 \& \text { fue } / / \mathrm{mo} \times \frac{(23 \mathrm{~kg} \mathrm{co}}{1000 \mathrm{l}} 1.3-2\right) \\
& \therefore \frac{1.022 \mathrm{~kg} \mathrm{CO} / \mathrm{mo}}{2465 \mathrm{~kg} \mathrm{U} / \mathrm{mo}}=4.15 \times 10^{-4} \frac{\mathrm{kg} \mathrm{CO}}{\mathrm{kg} \mathrm{U}}
\end{aligned}
$$

\section{B. 7.2.13 Hydrocarbons}

$$
\begin{aligned}
& \text { (23, p. 1.3-2) } \\
& 2044 \text { \& fuel } / \mathrm{mo} \times \frac{.35 \mathrm{~kg} \mathrm{C}_{x} \mathrm{H}_{x}}{1000 \text { 2 }}=.72 \mathrm{~kg} \mathrm{C} \mathrm{CH}_{x} / \mathrm{mo} \\
& \therefore \frac{.72 \mathrm{~kg} \mathrm{C}}{2465 \mathrm{~kg}} \times \frac{\mathrm{H}_{y} / \mathrm{mo}}{\mathrm{U} / \mathrm{mo}}=2.9 \times 10^{-4} \frac{\mathrm{kg} \mathrm{Hydrocarbons}}{\mathrm{kg} \mathrm{U}}
\end{aligned}
$$

B.7.2.14 Nitrogen Oxides

$$
\begin{aligned}
& 2044 \text { \& fuel } / \mathrm{mo} \times \frac{(23, \mathrm{~kg} \cdot 1.3-2)}{1000 \ell} \times=19.6 \mathrm{~kg} \mathrm{NO} \times \mathrm{mo} \\
& \therefore \frac{19.6 \mathrm{~kg} \mathrm{NO} \times}{24.65 \mathrm{~kg} \mathrm{U} / \mathrm{mo}}=7.96 \times 10^{-3} \frac{\mathrm{kg} \mathrm{NO}}{\mathrm{kg} \mathrm{U}} \times
\end{aligned}
$$

B. 7.2.15 Aldehydes

(23, p. 1.3-2)

2044 \& fuel $/ \mathrm{mo} \times \frac{.25 \mathrm{~kg} \mathrm{CH}}{1000 \text { \& }}=.511 \mathrm{~kg} \mathrm{CH} \mathrm{CH}_{2} \mathrm{O} / \mathrm{mo}$ 


$$
\therefore \frac{.511 \mathrm{~kg} \mathrm{CH}}{20 / \mathrm{mo}}=2.1 \times 10^{-4}
$$

\section{B.7.3 LIQUID RELEASES}

\section{B.7.3.1 Chiorine}

(21, p. III-46)

$.01 \mathrm{mg} / 2 \times 4.75 \times 10^{7} \mathrm{\ell} /$ day $\times \frac{\mathrm{kg}}{100 \mathrm{mg}}=.475 \mathrm{~kg} \mathrm{C1} /$ day

$\therefore \frac{.4 \% \mathrm{~kg} \mathrm{Cl} / \text { day }}{102 \mathrm{~kg} \mathrm{U} / \mathrm{day}}=4.7 \times 10^{-3} \frac{\mathrm{kg} \mathrm{Cl}}{\mathrm{kg} \mathrm{U}}$

\section{B.7.3.2 Prosphates}

(21, Table III-8)

$5 \mathrm{lb} \mathrm{PO}_{4} /$ day $\times \mathrm{kg} / 2.205 \mathrm{ib}=2.3 \mathrm{~kg} \mathrm{PO}_{4} /$ day

$$
\therefore \frac{2.3 \mathrm{~kg} \mathrm{PO}}{4} / \frac{\mathrm{day}}{102 \mathrm{~kg} \mathrm{~J} / \mathrm{day}}=2.2 \times 10^{-2} \frac{\mathrm{kg} \mathrm{PO}}{\mathrm{kg} \mathrm{U}}
$$

\section{B.7.3.3 Lithium}

(21, p. III-51) $.009 \mathrm{mg} / \ell \times 4.75 \times 10^{7} \ell /$ day $\times \frac{\mathrm{kg}}{10^{5} \mathrm{~kg}}=.4275 \mathrm{~kg} \mathrm{Li} /$ day $\therefore \frac{.4275 \mathrm{~kg} \mathrm{Li} / \text { day }}{102 \mathrm{~kg} \mathrm{U} / \text { day }}=4.2 \times 10^{-3} \frac{\mathrm{kg} \mathrm{Li}}{\mathrm{kg} \mathrm{U}}$

\section{B.7.4 Carbon 14}

Much of the ${ }^{14} \mathrm{C}$ is generated within the liquid coolant and some may be entrained in the blowdown as fine bubbles or dissolved in the liquid releases, so that it starts out as a liquid release. "Leakers" among the fuel rods either in the reactor core or in the storage basin tend to release part of the ${ }^{14} \mathrm{C}$ generated within the fuel. This also starts out entrained as a liquid release but is eventually released to the environment as an airborne release. A release factor of 0.30 was calculated as follows: 
From Appendix A, reference salculation 8-1:

$101,033 \mathrm{~kg} \mathrm{UO} \mathrm{O}_{2} \times \frac{238}{270} \times \frac{i}{3} \times \frac{1}{290}$ operating days $=102 \mathrm{~kg} \mathrm{U}$ per day The activity value is obtained from Reference 18 , page B-58, with the exception that we are using the NPC figure of $8 \mathrm{Ci}$ per year for the ${ }^{17} \mathrm{O}(\mathrm{n}, x){ }^{14} \mathrm{C}$ reaction in the coolant. We find no basis for deviating from 0.09 ci per year as calculated by the SAI study for the ${ }^{14} \mathrm{iN}(n, p){ }^{14} \mathrm{C}$ reaction in the PWR coolant:

$$
\begin{array}{lc}
\text { PWR Fuel }{ }^{17} O(n, \alpha){ }^{14} \mathrm{C} & 4.1 \\
\text { PWR Fuel }{ }^{14} \mathrm{~N}(n, p){ }^{14} \mathrm{C} & 17.3 \\
\text { PINR Coolant }{ }^{17} O(n, \alpha){ }^{14} \mathrm{C} & 8.0 \\
\text { PIN Coolant }{ }^{14} \mathrm{~N}(n, p){ }^{14} \mathrm{C} & \underline{0.09} \\
\quad \text { Total annual production } \\
{ }^{14} \mathrm{C} \text { in Ci/GWe-year } & 29.49
\end{array}
$$

A 1250 MWe reactor at $80 \%$ capacity factor produces 1,000 MWe-year/ year or one GWe-year. Therefore, $\mathrm{Ci} / \mathrm{GW}$-year is the same as $\mathrm{Ci}$ per operating year for a 1250 MWe reactor at $80 \%$. The reference facility assumes only $79.45 \%$ plant capacity factor or 290 days operation. Adjusting for this:

$$
29.49 \mathrm{Ci} / \text { year } \times \frac{79.45}{80.0}=29.29 \mathrm{Ci} / \text { year }
$$

Utilizing the specific activity of ${ }^{14} \mathrm{C}$ of $4.46 \mathrm{Ci} / \mathrm{gram}$ :

$$
\begin{aligned}
& \frac{29.29 \mathrm{Ci} / \text { year }}{4.46 \mathrm{Ci} / \text { gram }} \times \frac{1}{290 \text { days }} \times \frac{1}{1000 \mathrm{~g} \text { per } \mathrm{kg}}= \\
& \begin{array}{ll}
2.26 \times 10^{-5} \mathrm{~kg}{ }^{14} \mathrm{C} \text { per operating day } \\
\frac{2.26 \times 10^{-5} \mathrm{~kg}^{14} \mathrm{C} \text { per operating day }}{102 \mathrm{~kg} \mathrm{U} \text { per operating day }}= & 2.22 \times 10^{-7} \mathrm{~kg} \\
& \text { product per } \mathrm{kg} \mathrm{U} \text { input }
\end{array}
\end{aligned}
$$

Table 2-3, page 2-18 of the Bray-Miller-Rieke "Assessment of ${ }^{14} \mathrm{C}$ " document provides releases of gaseous Carbon 14 which we have revised as footnoted: 
Pathway

Gaseous radwaste treatment system

Condenser steam-jet air ejector offgas vent

Steam generator blowdown tank vent

Turbine gland seal condenser exhaust

Fuel handling building ventilation

Containment building purge exhaust

Auxiliary building ventilation

Turbine building ventilation

Total annual ${ }^{14} \mathrm{C}$ release
Annua? ${ }^{14} \mathrm{C}$ Release

Ci/year

cilyear

Footro=e

$4.5 \times 10^{-4}$

$9.2 \times 10^{-7}$

$8.0 \times 10^{-4}$

$1.8 \times 10^{-3}$

\subsection{1}

(1) See last paragraph page 2-47 and top of page 2-48, NUREG-0017.

(2) No reason found for changing this.

(3) This comes from "leakers" among the irradiated fuel elements and is not derived from irradiation of the coolant.

$\frac{8.81 \mathrm{Ci} / \text { year }}{4.46 \mathrm{Ci} / \text { gram }} \times \frac{1}{290 \text { days }} \times \frac{1}{1000 \mathrm{~g} \mathrm{per} \mathrm{kg}}=6.81 \times 10^{-6} \mathrm{~kg}$ ${ }^{14} \mathrm{C}$ per day released

$\frac{6.81 \times 10^{-6} \mathrm{~kg}{ }^{14} \mathrm{C} \text { per day }}{102 \mathrm{~kg} \mathrm{U} \text { per operating day }}=6.67 \times 10^{-8} \mathrm{~kg}{ }^{14} \mathrm{C}$ released per $\mathrm{kg} \mathrm{U}$ input Release factor $=\frac{6.67 \times 10^{-8} \mathrm{~kg}{ }^{14} \mathrm{C} \text { released per } \mathrm{kg} U \text { input }}{2.22 \times 10^{-7} \mathrm{~kg}{ }^{14} \mathrm{C} \text { produced per } \mathrm{kg} U \text { input }}$ $=0.30$ 
TABLE B-E. EoTing Nater Reactor Chemical Reiease Factors, $\mathrm{kg} / \mathrm{kg} U$ (input)

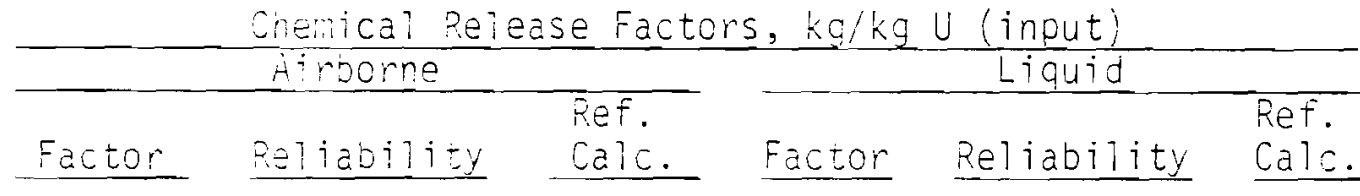

Reieased Material

Sulfur Oxides

0.3

2

8.2.11

iitrogen Oxides

Hydrocarbons

Particulates

chiorine

Calcium

Magnesium

Sodiumi

Bicürbonate

Chloride

Sulfate

Nitrate

Phosphate

silica

0.2

$3 \times 10^{-3}$

$7 \times 10^{-3}$

$6 \times 10^{-2}$

2

$4 \times 10^{-1}$

$2 \times 10^{-1}$

2

$9 \times 10^{-2}$

4

$2 \times 10^{-2}$

$1 \times 10^{-3}$

$5 \times 10^{-1}$

2

2

2

1

2

2

2

2

2

2

2

2

2
3.2 .12

8.2 .13

8.2 .14

8.2 .15

8.2 .1

8.2 .2

8.2 .3

8.2 .4

8.2 .5

8.2 .6

8.2 .7

8.2 .8

8.2 .9

Radionuclide Release Factor, $\mathrm{kg}$ Released/kg Radionuclide (input)

Carbon-14

$\begin{array}{lll}0.3 & 2 & 8.3\end{array}$




\section{B.9 BOILIIIG WATER REACTOR}

\section{3.? ASSUPTichs}

The following values were extracted from Calculation $9-1$ in Reference 1 :

- $84 \mathrm{~kg} \mathrm{u(input)/day}$

- 29? operating dajs/yr.

From the above data, the annual uranium input is $24,528 \mathrm{~kg}$.

A recirculating cooling tower system with mechanical draft wet cooling towers will dissipate excess heat to the atmosphere. A small quantity of water entrained in the air passing through the cooling tower is lost from the system as "drift." Drift eliminators are used in Hanford No. 2's cooling tower to minimize drift to approximately $285 \mathrm{gpm}$ or $1.55 \times 10^{6} \mathrm{~L} /$ day (Reference 25, p. 3.3-1). This drift is of the same composition as the circulating water and is assumed an airborne release (see Calculations 8.2.1 to 8.2.9).

\section{B.8.2 AIRBORNE RELEASES}

\section{B.8.2.1 Calcium}

(25, Table 3.6-1)

$116 \mathrm{mg} / \mathrm{2} \times 1.55 \times 10^{6} \mathrm{e} /$ day $\times \frac{\mathrm{kg}}{10^{0} \mathrm{mg}}=179.8 \mathrm{~kg} \mathrm{Ca} / \mathrm{day}$

$\therefore \frac{179.8 \mathrm{~kg} \mathrm{Ca} / \mathrm{day}}{84 \mathrm{~kg} \mathrm{U} / \mathrm{day}}=2.14 \frac{\mathrm{kg} \mathrm{Ca}}{\mathrm{kg} \mathrm{U}}$

\section{B.8.2.2 Magnesium}

(25, Table 3.5-1)

$21 \mathrm{mg} / \mathrm{i} \times 1.55 \times 10^{6} \mathrm{\ell} /$ day $\times \frac{\mathrm{kg}}{10^{0} \mathrm{mg}}=32.55 \mathrm{~kg} \mathrm{Mg} /$ day

$$
\therefore \frac{32.55 \mathrm{~kg} \mathrm{lig} / \mathrm{day}}{84 \mathrm{~kg} \mathrm{U} / \mathrm{day}}=.3875 \frac{\mathrm{kg} \mathrm{Mg}}{\mathrm{kg} \mathrm{U}}
$$

\section{B.8.2.3 Sodium}

(25, Table 3.6-1)

$12 \mathrm{mg} / \mathrm{z} \times 1.55 \times 10^{6} \mathrm{\ell} /$ day $\times \frac{\mathrm{kg}}{10^{6} \mathrm{mg}}=18.6 \mathrm{~kg} \mathrm{Na} / \mathrm{day}$ 


$$
\therefore \frac{13.5 \mathrm{~kg} \mathrm{Ha/day}}{3+\mathrm{kg} \mathrm{v/day}}=.22 \frac{\mathrm{kg} \% \mathrm{~kg}}{\mathrm{~kg}}
$$

\subsubsection{Eicarconate}

\section{(25, Table 3.6-7)}

$92 \mathrm{mg} / \hat{\mathrm{s}} \times 1.55 \times 10^{6} \mathrm{\ell} / \mathrm{day} \times \frac{\mathrm{kg}}{106 \mathrm{mg}}=142.6 \mathrm{~kg} \mathrm{\textrm {HCO } _ { 3 }}{ }^{-} / \mathrm{day}$

$\therefore \frac{142.6 \mathrm{~kg} \mathrm{HCO} 3^{-} / \mathrm{day}}{84 \mathrm{~kg} \mathrm{U} / \mathrm{day}}=1.7 \frac{\mathrm{kg} \mathrm{HCO}^{-}}{\mathrm{kg} \mathrm{U}^{-}}$

\subsubsection{Chioride}

\section{(25, Tabte 3.6-1)}

$5 \mathrm{mg} / \mathrm{z} \times 1.55 \times 10^{6} \mathrm{\ell} /$ day $\times \frac{\mathrm{kg}}{108 \mathrm{mg}}=7.75 \mathrm{~kg}$ Chloride/day

$\therefore \frac{7.75 \mathrm{~kg} \text { Chloride/day }}{84 \mathrm{~kg} \mathrm{U} / \mathrm{day}}=.093 \frac{\mathrm{kg} \mathrm{Chloride}}{\mathrm{kg} \mathrm{U}}$

\section{B.8.2.6 Sulfate}

(25, Table 3.6-1)

$236 \mathrm{mg} / \hat{\imath} \times 1.55 \times 10^{6} \mathrm{z} /$ day $\times \frac{\mathrm{kg}}{10^{0} \mathrm{mg}}=365.8 \mathrm{~kg} \mathrm{SO} 4 /$ day

$\therefore \frac{365.8 \mathrm{~kg} \mathrm{SO}}{84 \mathrm{~kg} \mathrm{U} / \mathrm{day}}=4.35 \frac{\mathrm{kg} \mathrm{SO}}{\mathrm{kg} \mathrm{\textrm {d } ^ { - }}}$

\subsubsection{Nitrate}

(25, Table 3.6-i)

$1.24 \mathrm{mg} / \hat{2} \times 1.55 \times 10^{6} \mathrm{l} / \mathrm{day} \times \frac{\mathrm{kg}}{10^{0} \mathrm{mg}}=1.922 \mathrm{~kg} \mathrm{NO} /$ day

$\therefore \frac{1.922 \mathrm{~kg} \mathrm{NO} 3 / \text { day }}{84 \mathrm{~kg} \mathrm{~V} / \mathrm{day}}=.023 \frac{\mathrm{kg} \mathrm{NO}}{\mathrm{kg} \mathrm{V}} 3$

\section{B.8.2.8 Phosphate}

(25, Table 3.6-1)

$.05 \mathrm{mg} / 2 \times 1.55 \times 10^{6} \mathrm{\ell} /$ day $\times \frac{\mathrm{kg}}{10^{6} \mathrm{mg}}=.093 \mathrm{~kg} \mathrm{PO} /$ day 


$$
\therefore \frac{.093 \mathrm{kgPO} / \mathrm{day}}{84 \mathrm{~kg} \mathrm{U} / \mathrm{day}}=1.1 \times 10^{-3} \frac{\mathrm{kg} \mathrm{PO}}{\mathrm{kg} \mathrm{U}^{4}}
$$

\section{3 .2 .3 Silica}

\section{5, Table 3.6-1}

$30 \mathrm{mg} / 2 \times 1.55 \times 10^{6} \mathrm{z} /$ day $\times \frac{\mathrm{kg}}{100 \mathrm{mg}}=46.5 \mathrm{~kg} \mathrm{Silica/ \text {day }}$

$\therefore \frac{46.5 \mathrm{~kg} \mathrm{Silica/day}}{84 \mathrm{~kg} U / \text { day }}=.55 \frac{\mathrm{kg} \text { Silica }}{\mathrm{kgU}}$

\subsubsection{Combustion Products}

Three standby diesel engines will be test run for about $2 \mathrm{hr} / \mathrm{mo}$. Two will consume $340 \mathrm{gph}$ of fuel each. The third wil1 use $170 \mathrm{gph}(25, \mathrm{p} .3 .7-1)$. The engines burn No. 2 fuel $0 i 1(26, p .2 .3 .4 .3)$ and will exhaust annually the following effluents $(25$, p. $3.7-1)$ :

$$
\begin{array}{lr}
\mathrm{SO}_{2}^{-} & 1,4001 \mathrm{~b} \\
\mathrm{NO}^{2} & 9801 \mathrm{~b} \\
& 341 \mathrm{~b} \\
\text { Hydrocarbons } & 4121 \mathrm{~b}
\end{array}
$$

The heating boiler-also consumes No. 2 fuel oil containing $0.4 \%$ sulfur at a rate of $432 \mathrm{gph}$ and will produce annually: (25)

$$
\begin{array}{lr}
\mathrm{SO}_{2} & 13,650 \mathrm{~Tb} \\
\mathrm{NO}_{x} & 9,800 \mathrm{ib} \\
\text { Particulates } & 340 \mathrm{ib}
\end{array}
$$

\section{B.8.2.11 Sulfur Oxides}

$$
\begin{aligned}
& (1,400) 1 \mathrm{~b}+13,6501 \mathrm{~b}) / \mathrm{yr} \times 1 \mathrm{~kg} / 2.2051 \mathrm{~b}=6,825.4 \mathrm{~kg} \mathrm{SO} / \mathrm{yr} \\
& \therefore \frac{6,825 \mathrm{~kg} \mathrm{SO} 2 / \mathrm{yr}}{24,528 \mathrm{~kg} \mathrm{U} / \mathrm{yr}}=0.278 \frac{\mathrm{kg} \mathrm{SO} 2}{\mathrm{~kg} \mathrm{U}}
\end{aligned}
$$

\section{B.8.2.12 Nitrogen Oxides}

$(980 \mathrm{lb}+9,800 \mathrm{lb}) / \mathrm{yr} \times \mathrm{kg} / 2.205 \mathrm{7b}=4,889 \mathrm{~kg} \mathrm{NO} / \mathrm{yr}$

$$
\therefore \frac{4,889 \mathrm{~kg} \mathrm{NO} / \mathrm{yr}}{24,528 \mathrm{~kg} \mathrm{U} / \mathrm{yr}}=0.199 \frac{\mathrm{kg} \mathrm{NO}}{\mathrm{kg} \mathrm{U}}
$$




\subsubsection{Hydrocarbons}

$41216 H_{x} C_{x} / y r \times i \mathrm{~kg} / 2.20516=187 \mathrm{~kg} H_{x} C_{x} / y r$

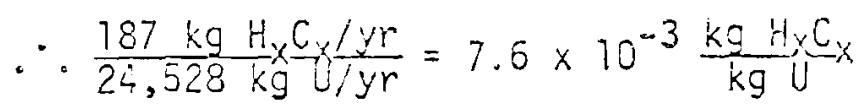

3.3.2.74 Particulates

$$
\begin{aligned}
& 34+340 \mathrm{lb} / \mathrm{yr} \times \mathrm{kg} / 2.205 \mathrm{lb}=170 \mathrm{~kg} \text { Particulates } / \mathrm{yr} \\
& \therefore \frac{170 \mathrm{~kg} / \mathrm{yr}}{24,528 \mathrm{~kg} U / \mathrm{yr}}=6.9 \times 10^{-3} \frac{\mathrm{kg} \text { Particulates }}{\mathrm{kg} \mathrm{U}}
\end{aligned}
$$

\section{B.8.2.15 Chlorine}

Approximately $240 \mathrm{lb} /$ day of chlorine is added to the circulating water line upstream of the main condenser. A small portion of this will be dispersed to the atmosphere and the remainder consumed $(25, p .3 .6-2)$. In absence of other data, a loss of $5 \%$ shall be assumed:

$240 \mathrm{lb} /$ day $\times .05$ loss $\times \mathrm{kg} / 2.205 \mathrm{lb}=5.44 \mathrm{~kg} \mathrm{Cl} /$ day

$\therefore \frac{5.44 \mathrm{~kg} \mathrm{Cl} / \mathrm{day}}{84 \mathrm{~kg} \mathrm{U} / \text { day }}=.065 \frac{\mathrm{kg} \mathrm{Cl}}{\mathrm{kg} \mathrm{U}}$

\section{B.8.3 CARBON-14}

A release factor of 0.294 was derived as follows: Calculation 9-1 of Appendix A provided the daily throughput,

$139,344 \mathrm{~kg} \mathrm{U0} 2 \times \frac{238 \mathrm{U}}{270 \mathrm{UO}_{2}} \times \frac{1}{5} \times \frac{1}{292 \text { days }}=84 \mathrm{~kg}$ U per day

The activity value is obtained from Reference 18, p. 2-9:

$$
\begin{aligned}
& \text { BWR Fuel }{ }^{17} \mathrm{O}(\mathrm{n}, \mathrm{a}){ }^{14} \mathrm{C} \quad 4.1 \\
& \text { BWR Fuei }{ }^{14} \mathrm{~N}(n, p){ }^{14} \mathrm{C} \quad 17.3 \\
& \text { EWR Coolant }{ }^{17} \mathrm{O}(n, a){ }^{14} \mathrm{C} \quad 9.2 \\
& \text { BiNR Coolant }{ }^{14} \mathrm{~N}(n, p){ }^{14} \mathrm{C} \quad \underline{0.26} \\
& \text { Total Annual Production }{ }^{14} \mathrm{C} \\
& \text { in } \mathrm{Ci} / \mathrm{GW} \text {-year } \quad 30.36
\end{aligned}
$$


A 1250 We reactor at 80 . cazacity factor produces 1000 MMe-year/year or one Gile-year. Therefore, Ci/Gi-year is the same as Ci per operating year foi a 7250 : Whe at $30 \%$. Then, litilizing the specific activity of ${ }^{14} \mathrm{C}$ of $4.45 \mathrm{Ci} / \mathrm{cram}$ :

$$
\begin{aligned}
& \frac{30.86 \mathrm{Ci} / \text { year }}{4.46 \mathrm{Ci} / \mathrm{gram}} \times \frac{1}{292 \text { days }} \times \frac{1}{1000 \mathrm{~g} \mathrm{per} \mathrm{kg}} \\
& =2.37 \times 10^{-5} \mathrm{~kg}{ }^{14} \mathrm{C} \text { per operating day } \\
& \frac{3.37 \times 10^{-5} \mathrm{~kg}^{14} \mathrm{C} \text { per operating day }}{34 \mathrm{~kg} \mathrm{~V} \text { per operating day }}=\frac{2.82 \times 10^{-7}}{\mathrm{~kg} \mathrm{U} \text { input }} \mathrm{kg}^{14} \mathrm{C} \text { per }
\end{aligned}
$$

Table 2-2, Page 2-12 of Reference 18 provides BWR releases of gaseous Carbon-14:

$$
\begin{aligned}
& \text { Annuar }{ }^{14} \mathrm{C} \text { Release } \\
& \frac{9.056 \mathrm{Ci} / \text { vear }}{4.46 \mathrm{Ci} / \mathrm{gram}} \times \frac{1}{292 \text { days }} \times \frac{1}{1000 \mathrm{~g} \text { per } \mathrm{kg}}=9,96 \times 10^{-6} \mathrm{~kg} \text { per day }
\end{aligned}
$$

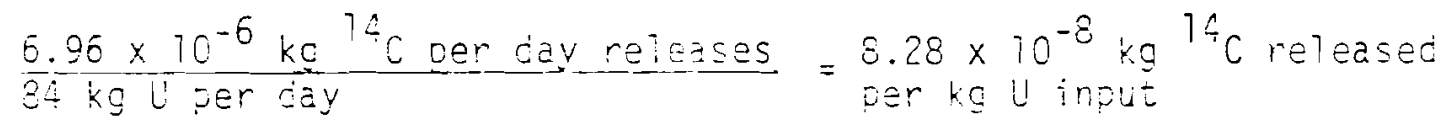

$$
\begin{aligned}
& \text { Release factor }=\frac{8.28 \times 10^{-8}}{2.32 \times 10^{-14} \mathrm{~kg} \text { in } \mathrm{C} \text { reieased per } \mathrm{kg} \mathrm{U} \text { input }} \\
& =0.294
\end{aligned}
$$


Aiso, the $9.064 \mathrm{Ci} / \mathrm{yr}$ is in good agrement with measurements at line $14 i 7 \mathrm{l}$ Point 1 reported by C. O. Kunz, M. E. Mahoney, and T. M. Mitier of the Radiation Survey Laboratories, N. Y. State Departiment of Health in their paper "Carbon-14 Gaseous Effluents from Boiling liater Reactors." We therefore feel that our factor of 0.294 for B:iR can be considered of Level 2 reliability.

TABLE B-Q. Fuel Reprocessing

Chemical Release Factors, $\mathrm{kg} / \mathrm{kg} U$ (input)

- Airborne - Lef. - Liquid - - -

Material Released Factor Reliability Calc. Factor Reliability Calc.

Fluorine $1 \times 10^{-4}$

9.2 .1

Sodium

Chroride

$1 \times 10^{-1} \quad 1$

9.3 .1

$7 \times 10^{-3}$

9.3 .2

Radionuclide Release Factor, $\mathrm{kg}$ released/ $\mathrm{kg}$ radionuclide (input)

Carbon 14

1.0

2

9.4 


\subsection{FLEL REPROCESS:IG}

\section{9 .7 ASSMATIONS}

The following effluents were listed in Reference 4 for tne WASH-1248 model fuel reprocessing plant. The effluent data below was normalized to the 900 MTU/yr capacity of the WASH 1248 model planit. Reiease factors shall be calculated directly from this data.

\section{B.9.2 AIRBORNE RELEASE}

\subsubsection{Fluorine}

$(4, p . F-2 i)$

$\frac{.7 \mathrm{HT} F / \mathrm{yr}}{900 \mathrm{MUU} / \mathrm{yr}}=1.1 \times 10^{-4} \frac{\mathrm{MTF}}{\mathrm{MTU}}=1.1 \times 10^{-4} \frac{\mathrm{kgF}}{\mathrm{kgU}}$

\section{B.9.3 LIQUID RELEASES}

\section{B.9.3.1 Sodium (water)}

(4, p. F-22)

$$
\frac{137 \mathrm{MT} \mathrm{Na} / \mathrm{yr}}{900 \mathrm{MU} / \mathrm{yr}}=1.5 \times 10^{-1} \frac{\mathrm{MT} \mathrm{Na}}{\mathrm{MTU}}=1.5 \times 10^{-1} \frac{\mathrm{kg} \mathrm{Na}}{\mathrm{kg} \mathrm{U}}
$$

\section{B.9.3.2 Chloride (water)}

$(4, p . F-22)$

\section{$\frac{5.3 \mathrm{MT} \text { Chloride/yr }}{900 \text { MTU } / \mathrm{yr}}=7 \times 10^{-3} \frac{\text { HT Chloride }}{\text { MTU }}=7 \times 10^{-3} \frac{\mathrm{kg} \text { Chloride }}{\mathrm{kg} \mathrm{U}}$}

\section{B.9.4 CARBON 14}

This is a mixture of liquid and airborne release but eventually becomes entirely airborne. A release factor of 1.0 (unity) for the ${ }^{14} \mathrm{C}$ released from the reference fuel reprocessing facility was derived as follows: 


\section{Assumptions}

1. The reference reprocessing facility will serve a mix of one-thrid BURS and two-thirds pirks (measured in gross itwe piant capacicy).

2. The irradiated fuel wiil remain an average of six months in the irradiated fuel storage basins at the reactors $\left({ }^{14} \mathrm{C}\right.$ from leaking fue pins was already accounted for in our Calculations 7.4 and 8.3).

3. The time from reactor discharge to reprocessing dissolver will average $1-1 / 2$ years.

4. The fuel casks are always sealed in transit. Al $1{ }^{14} \mathrm{C}$ evolved from leaker fuel pins in transit is therefore part of reprocessing plant accountability. The casks are solidly connected to the low-level gas system before they are vented and flushed through the radwaste system and eventually vented through the 100 meter stack at the reprocessing plant.

5. No equipment will have been installed as yet for the capture of ${ }^{14} \mathrm{C}$ when the reprocessing plant is initially operated, which is the situation we are appraising.

\section{Calculations}

The annual contribution of ${ }^{14} \mathrm{C}$ received in the irradiated fuel shipped from a 1250 MWe PWR is computed as follows (see Calculation 7.4):

$22.2 \times 10^{-8} \mathrm{~kg}{ }^{14} \mathrm{C}$ produced per $\mathrm{kg} \mathrm{U}$ Input

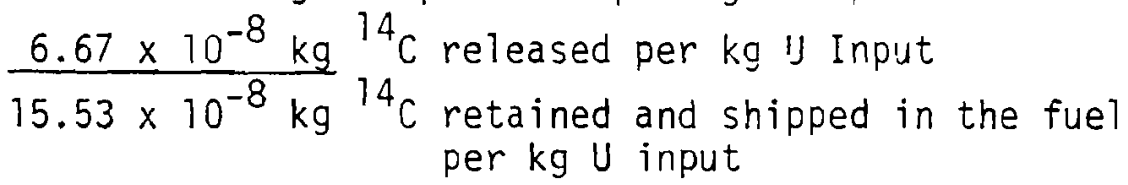

From Appendix A, reference Calculation 8-1 and Section 11A Pressurized Water Reactor, page 11-2, we assume $1 / 3$ of fuel is changed out each year:

$101,033 \mathrm{~kg} \mathrm{J0_{2 }} \times \frac{238}{270} \times \frac{1}{3}=29,686 \mathrm{~kg} /$ year discharged $15.53 \times 10^{-8} \mathrm{~kg}{ }^{14} \mathrm{C}$ per year $\mathrm{kg} \mathrm{U}$ input $\times 29,686 \mathrm{~kg} /$ year discharged $=4.61 \times 10^{-3} \mathrm{~kg}{ }^{14} \mathrm{C}$ per year shipped with the irradiated PWR fuel. 
The annual contribution of ${ }^{14} \mathrm{C}$ received in the irradiated fuel sniposd from a 1250 . We BWR is computed as follows (see Reference Calculation 8.3):

$28.2 \times 10^{-8} \mathrm{~kg}^{14} \mathrm{C}$ produced per $\mathrm{kg} \mathrm{U}$ input $\frac{8.28 \times 10^{-8} \mathrm{~kg}}{19.92 \times 10^{-8} \mathrm{~kg}}{ }^{14} \mathrm{C}$ released per $\mathrm{kg} U$ input

From Appendix A, Reference Caiculation 9.1, and Section 17B, page 11-45, we assume $1 / 5$ of the fuel is changed out each year:

$139,344 \mathrm{~kg} \mathrm{LO} 2 \times \frac{238}{270} \times \frac{1}{5}=24,565.83 \mathrm{~kg} /$ year $U$ discharged

$19.92 \times 10^{-8} \mathrm{~kg}{ }^{14} \mathrm{C}$ per $\mathrm{kg} U$ fuel discharged $\times 24,565.83 \mathrm{~kg} /$ year

$U$ discharged $=4.89 \times 10^{-3} \mathrm{~kg}{ }^{14} \mathrm{C} /$ year shipped with the irradiated $B W R$ fuel

In accordance with our assumption of a mix of two 1250 MWe PWR reactors for each BWR reactor:

29.686 MT/year $U$ from 1250 MWe PWR and $4.61 \times 10^{-3} \mathrm{~kg}{ }^{14} \mathrm{C}$ in fuel $29.686 \mathrm{MT}$ /year $U$ from $1250 \mathrm{MWe} P W R$ and $4.61 \times 10^{-3} \mathrm{~kg}{ }^{14} \mathrm{C}$ in fue $24.566 \mathrm{MT} /$ year $U$ from $1250 \mathrm{MWe} B W R$ and $\underline{4.89 \times 10^{-3}} \mathrm{~kg}{ }^{14} \mathrm{C}$ in fuel $83.938 \mathrm{WT} /$ year $U$ total mix of $\quad 14.11 \times 10^{-3} \mathrm{~kg}{ }^{14} \mathrm{C}$ arriving three reactors in fue 1

$27.98 \mathrm{MT} /$ year $U$ average per 1250 MWe reactor $4.70 \times 10^{-3} \mathrm{~kg}{ }^{14} \mathrm{C}$ arriving in fuel

From Section 12, page 12-1, the reference LWR fuel reprocessing faci1ity has an annual capacity of 1500 MTU/year (as charged into a reactor);

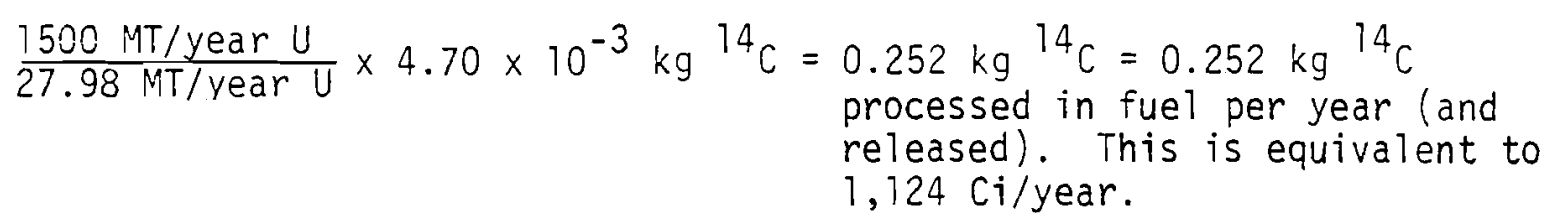


Section 72 plovided a separate, ingependent calculation of ${ }^{14} \mathrm{C}$ released based

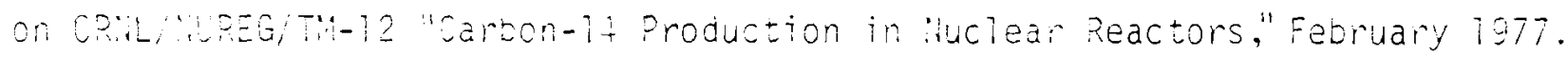

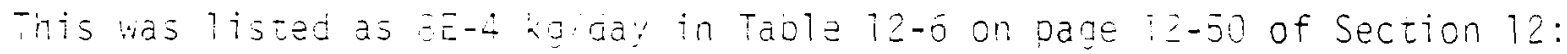

$$
\Xi \times 10^{-4} \mathrm{~kg} / \mathrm{day} \times 300 \text { production days }=0.240 \mathrm{~kg}{ }^{14} \mathrm{C} \text { released per year }
$$

This is a reasonabiy close check to our $0.252 \mathrm{~kg}{ }^{14} \mathrm{C}$ which we derived in this Appendix $B$.

The following table of pathways for releases is little more than a guess, since, without an oserating reprocessing plant, it has not been possible to actually measure ${ }^{14} \mathrm{C}$ effluents:

$\begin{array}{lc}\text { Pathway } & 1 \\ \text { Fuel receiving and storage } & 25 \\ \text { Shearing of fuel assemblies } & 49 \\ \text { Dissolution of fuel } & \\ \begin{array}{l}\text { Solvent extraction, partitioning } \\ \text { and stripping }\end{array} & \underline{25} \\ & 100\end{array}$

In accordance with our Assumption 5, gaseous effluent from each of these pathways would be collected and would eventually be exhausted from the 100 meter stack as part of the 3115 cubic meters per minute flow $(110,000 \mathrm{cfm})$.

All of the knowledgeable people we have consulted are of the opinion that al1 of the ${ }^{14} \mathrm{C}$ is released in gaseous forms as $\mathrm{CO}, \mathrm{CO}_{2}, \mathrm{CH}_{4}, \mathrm{C}_{2} \mathrm{H}_{6}$, and a minor amount in other gases, all of which would be released through the 100 meter stack. Willis Davis, Jr., in ORNL/NUREG/TM-12, "Carbon 14 Production in Nuclear Reactors" on "Abstract" page, says". . However, some small fraction may remain in aqueous raffinates and will not be released until these are converted to solids.. ." We agree this is possibly true. However, the waste solidification process is now a required part of a reprocessing plant. This small fraction, if it exists, is merely a delayed release in the same plant. ORNL/NUREG/TM-12 also points out: "Cladding metal and other core hardware 
"ill contain significant quantities of ${ }^{7 \hat{4}} \mathrm{C}$. Very ittte of this will be released from BHR, PIR, and UHrsR nardiare at fuel reprocessing plants; instead, the contained ${ }^{14} \mathrm{C}$, 30 to 60 CijGw(e)-year for Lwis. . . wiil remain Within the metal, which will be retained onsite or in a federal repository." Our figures have not dealt with this 30 to $60 \mathrm{Ci} /$ year, since it is not avai?able as either a gas or a i iquid effluent at either the reactors or the fuel reprocessing piant. We therefore can conclude that the ${ }^{14} \mathrm{C}$ release factor for the LWR Reprocessing Plant is unity:

Release factor $=\frac{0.252 \mathrm{~kg}{ }^{14} \mathrm{C} \text { released to environment } / \text { year }}{0.252 \mathrm{~kg}^{14} \mathrm{C} \text { received in fuel/year }}=1$

NUTE: If the 30 to $60 \mathrm{Ci} /$ wive-year ${ }^{14} \mathrm{C}$ contained in the cladding rietals and other core hardware could have, somehow, been entirely released in the reprocessing plant:

$60 \mathrm{Ci} /$ year $\times \frac{1500 \mathrm{MT} / \text { year } \mathrm{U} \text { reprocessing plant capacity }}{27.98 \mathrm{MT} / \text { year U per reactor }}=3216 \mathrm{Ci} /$ year $\frac{3215 \mathrm{Ci} \text { of }{ }^{14} \mathrm{C}}{\therefore .46 \mathrm{Ci} / \text { gram }^{14} \mathrm{C}}=721.2$ gram $=0.721 \mathrm{~kg}^{14} \mathrm{C}$ $30 \mathrm{Ci} /$ year $\times \frac{1500 \mathrm{MT} / \text { year } \mathrm{U}}{27.98 \mathrm{MT} / \text { year } \mathrm{U} / \text { reactor }} \times \frac{1}{4.46 \mathrm{Ci} / \text { year }}=0.360 \mathrm{~kg}{ }^{14} \mathrm{C}$

Any such amounts, or any portion thereof, if released would almost certainly form a gaseous compound, be released, so that the release factor would still be 1 (unity). 



\section{REFERENCES}

1. Appendix A.

2. U. A. Cooper et a1. Characteristics of Attached Radon-222 Daughters Urider Both iaboratory and Field Conditions with Particular Emphasis Upon Underground Uranium Mine Environments." BN-SA-299, USBM Contract Report (HO 220029), December 1973.

3. Rio Algom Corp., Applicant's Supplemental Environmental Report, Operating License Stage for Uranium Concentrator. Moab, Utah, submitted to U.S. Atcmic Energy Commission, Docket No. 40-4084-2, November 1971.

4. U. S. Atomic Energy Commission, Environmental Survey of the Uranium Fuel Cycle. WASH-1248, Directorate of Licensing, Fuels and Materials, Apri1 1974.

5. Development Document for Interim Final and Proposed Effluent Limitations Guidel ines and New Source Performance Standards for the Ore Mining and Dressing Industry. Point Source Category, Vol. I, U. S. Environmental Protection Agency, Washington, DC, October 1975.

6. R. C. Merritt. The Extractive Metallurgy of Uranium. Prepared for Colorado School of Mines Institute for the U. S. Atomic Energy Commission, 1971.

7. E. C. Tsivoglou and R. L. O'Connel1. Vaste Guide for The Uranium Milling Industry. U. S. Department of Health, Education and Welfare, Public Health Service, Technical Report W62-12, 1962.

8. U. S. Atomic Energy Commission, Statistical Data of the Uranium Industry, 650-100, Grand Junction, Colorado, January 1, 1972.

9. M. B. Sears et al. Correlation of Radioactive Waste Treatment Costs and the Environmental Impact of Waste Effluents in the Nuclear Fuel cycle for Use in Establishing "As Low as Possible" Guide - Mlllling or Uranium Ores. ORNL-TM-4903, Vol. I, Oak Ridge, TN, May 1975.

10. Applicant's Environmental Report, Highland Uranium Mi11, Converse County, WY. Submitted by Humble $0 i 1$ and Refining Company, Mineral Department, Houston, TX, July 1971.

11. W. B. Harris et a1. Environmental Hazards Associated with the Milling of Uranium Ore - A Summary Report. U. S. AEC Report, HASL-40 (Rev.), New York Operations Office, Health, and Safety Laboratory, Revised November 14, 1958.

12. AEC Draft Environmental Statement, Sequoyah Uranium Hexafluoride Plant, Kerr-McGee Corporation, Docket No. 40-8027-11, April 1974. 
33. ․ J. Shelley, Kerr-ilcGee Nuclear Corporation, Oklahoma City, ok. Letter and attachment to Richard B. Chitwood. Nuclear Regulatory Commission, Public Document Rocm, Washington $\mathrm{CC}$. June 5, 1975.

: $\quad$. S. Fuclear Regulatory Comission's Final Environmental Statement. Sequoyan Uranium Hexafluoride Plant, Kerr-McGee Corporation, Docket io. 40-8027-12, February 1975.

15. $\because .3$. Shelley, Kerr McGee Nuclear Corporation, Oklahoma City, OK. Letter and attachnent to Richard B. Chitwood. Nuclear Regulatory Commission, Public Document Room, Washington DC. June 27, 1975.

16. Environmental Survey of the Uranium Fuel Cycle. USAEC, WASH 1248 , Apri1 1974.

17. Final Environmental Statement, Portsmouth Gaseous Diffusion Plant Expansion. ERDA, ERDA 1543.

18. G. R. Bray, L. Mitier, and J. W. Rieke. Assessment of ${ }^{14} \mathrm{C}$ Control Technology and Costs for the LWR Fuel Cycle. E.P.A. - SAI 70-593-WA. Science Applications, Icn.

19. NEDO-20197, ENvironmental Report, General Electric Nuclear Facility, Wilmington, North Carolina, General Electric Company, January 1974.

20. W. J. Goodwin. Westinghouse Nuclear Fuel Division, Columbia, SC. Letter to Leroy Person, NRC, Washington, DC, USAEC Docket No. 70-1151, dated December 1975.

21. Final Environmental Statement. Trojan Nuclear Plant Docket 50-344-66, August 1973.

22. Environmental Report. Trojan Nuclear Plant, USAEC, Docket No. 50-334, November 1971.

23. Compilation of Air Pollutant Emission Factors. US EPA, AP-42, Apri1 1973.

24. Perry and Chilton. Chemical Engineers Handbook. 5th Ed., McGraw-Hill, 1973.

25. Environmental Report. Operating License Stage, WPPSS Nuclear Project No. 2 USNRC Docket 50-397, March 1977.

26. Environmental Statement. Hanford No. 2, WPPSS, Docket No. 50-397, August 19, 1971.

27. Calculations of Releases of Radioactive llaterials in Gaseous and Liquid Effiuents from Pressurized Water Reactors (PWR-GALE Code). NUREG-0017, Office of Standards Development, U. S. Nuclear Regulatory Commission. April 1976. 

APPENDIX C

RELEASE RATES FOR A U02 FUEL FABRICATION FACILITY

\author{
R.L. McKeever \\ W.A. Muckerheide
}

The following tables give the chemical release rates from the Wilmington General Electric $\mathrm{UO}_{2}$ fuel fabrication facility (NEDO-20197). Some of these release rates are used in release factor calculations in Appendix $B$. 
TABLE C-1. Airborne-Chemical: General Electric

\begin{tabular}{|c|c|c|c|c|c|c|c|c|c|c|}
\hline - Efffluent & $\begin{array}{l}\text { Emission } \\
\text { Point } \\
\end{array}$ & Source & Particulate? & Soluble? & $\begin{array}{l}\text { Cheнi ical } \\
\text { Form } \\
\end{array}$ & $\begin{array}{l}\text { Release }(d) \\
\text { Rate } \\
\text { (kg/MIU) }\end{array}$ & $\begin{array}{l}0 \mathrm{i} \text { Index } \\
\left(\operatorname{lin}^{3} / M \times U\right)\end{array}$ & $\begin{array}{l}\text { Toxicity } \\
\left.\begin{array}{c}\mathrm{MPC} \\
(\mathrm{mug} / \pi !\end{array}\right)\end{array}$ & Basis & Ref. \\
\hline $\begin{array}{l}\text { Nitrogen } \\
\text { oxides }\end{array}$ & Stack & $\begin{array}{l}\text { Cbemical conver- } \\
\text { sion, calciner } \\
\text { barriers. } \\
\text { boilers, metal } \\
\text { clearing and } \\
\text { plating. } \\
\text { vorcinerator (R) }\end{array}$ & No & Yes & $\begin{array}{l}\mathrm{NO}_{\mathrm{x}} \\
(\text { mostly } \\
\mathrm{HO}_{2}\end{array}$ & 54.1 & $6.01 \mathrm{~EB}$ & .09 & A-7 & I \\
\hline Hydrocarbons & " & $\begin{array}{l}\text { Calcinator } \\
\text { burners, boilers }\end{array}$ & No & No & ${ }^{H} C_{y}$ & .06 & & & & \\
\hline $\begin{array}{l}\text { Carbon } \\
\text { (black) }\end{array}$ & $"$ & $\begin{array}{l}\text { Calcinator } \\
\text { burners, boilers }\end{array}$ & No & No & $\mathrm{CO}_{2}$ & .85 & $2.43 \mathrm{kI}$ & . & A & 1 \\
\hline $\mathrm{F}$ & $"$ & $\begin{array}{l}\text { Chemicol } \\
\text { conversion }\end{array}$ & No & $\begin{array}{c}\text { Yes } \\
(\text { both })\end{array}$ & $\mathrm{F}, \mathrm{F}_{2}$ & .0031 & $1.55 \mathrm{t5}$ & .02 & A & 1 \\
\hline $\begin{array}{l}\text { I, } 1,1 \\
\text { (yapor } \\
\text { degreaser) }\end{array}$ & " & $\begin{array}{l}\text { Yapor } \\
\text { degreaser }\end{array}$ & No & & & $5.4 \mathrm{H}$ & & & & \\
\hline $2,0_{2}$ & “ & lncinerator & No & No & $2 r, 0_{2}$ & 5.43 & 1.0958 & .05 & A & 1 \\
\hline $\begin{array}{l}\text { [thyl } \\
\text { acetate }\end{array}$ & " & Lab hoods & No & Stight & & 5.218 & $3.91[4$ & 14 & A & 1 \\
\hline Nitric acid & $"$ & Lab hoods & No & Yes & $\mathrm{HNO}_{3}$ & 3.48 & 1.1011 & .05 & A & ! \\
\hline Amovinia & $"$ & Waste treatment & No & Yes & $\mathrm{HAH}_{3}$ & 822 & 4. $36[9]$ & .145 & A & 1 \\
\hline $\begin{array}{l}\text { Boron } \\
\text { carbide }\end{array}$ & $"$ & Absorber rods & No & No & $B_{4} \mathrm{C}$ & .164 & $1.64 \mathrm{F.7}$ & .10 & A. 13 & I \\
\hline xylol & $"$ & Paint booth & No & & & 3.83 & $8.80 \mathrm{ES}$ & 4. 35 & A & 1 \\
\hline Butanol & Stack & Paint booth & No & Yes & $\mathrm{CH}_{2} \mathrm{Clt}_{2} \mathrm{CH}_{2} \mathrm{CH}_{2}$ & 4. 34 & 2.92 to & 1.5 & A & 1 \\
\hline Methyl butyl & $"$ & Paint booth & No & Stighe & & 2.74 & 6. $68 \mathrm{ES}$ & 4.1 & A & 1 \\
\hline $\begin{array}{l}\text { Glycol ethyl } \\
\text { ether }\end{array}$ & " & Paint booth & No & Very slight & & 2.19 & 5.92. L & 3.7 & A & 1 \\
\hline $\begin{array}{l}\text { Isopropyl } \\
\text { alcohot }\end{array}$ & " & Paint booth & No & Yes & & 13.7 & $1.40 \mathrm{~Eb}$ & 9.8 & A & 1 \\
\hline Sawdust & " & Hoodwork ing & Yes & & & & & & & \\
\hline $\mathrm{CrO}_{3}$ & $"$ & Chrome plat ing & No & $\begin{array}{l}\text { Ho, but yes } \\
\text { if in } \mathrm{Na} \text {. } \\
\mathrm{CR}_{2} \mathrm{O}_{7} \mathrm{form}\end{array}$ & $\mathrm{CrO}_{3}$ & .0228 & 2.2887 & .001 & A & i \\
\hline $\begin{array}{l}\text { Sulfur } \\
\text { dioxide }\end{array}$ & & & & Yes & $\mathrm{SO}_{2}$ & & & .13 & A & I \\
\hline
\end{tabular}


TABLE C-2. Liquid-Chemical: General Electric

\begin{tabular}{|c|c|c|c|c|c|c|c|c|c|c|}
\hline \multirow[b]{2}{*}{ Effluent } & \multirow[b]{2}{*}{$\begin{array}{l}\text { Emission } \\
\text { Point } \\
\end{array}$} & \multirow[b]{2}{*}{ Source } & \multirow[b]{2}{*}{ Particulate? } & \multirow[b]{2}{*}{ Soluble? } & \multirow[b]{2}{*}{$\begin{array}{l}\text { Chemical } \\
\text { Form } \\
\end{array}$} & \multirow{2}{*}{$\begin{array}{c}\text { Release } \\
\text { Rate } \\
(\mathrm{kg} / \mathrm{MTU}) \\
\end{array}$} & \multicolumn{4}{|c|}{ Toxicity } \\
\hline & & & & & & & $\begin{array}{l}\text { Dil. Index } \\
(\ell / \mathrm{MTU})\end{array}$ & $\begin{array}{c}M P C \\
(\mathrm{mg} / \mathrm{l})\end{array}$ & Basis & Ref.: \\
\hline $\mathrm{F}$ & $\begin{array}{l}\text { Holding } \\
\text { lagoon } \\
\text { overflow }\end{array}$ & Process waste & No & Yes & $\mathrm{F}^{-}$ & 2.9 & $2.90 E 6$ & 1 & $E, F$ & I I \\
\hline Ammonia & $"$ & $"$ & No & Yes & $\mathrm{NH}_{3}^{-}, \mathrm{NH}_{4}^{+}$ & 4.8 & $4.80 \mathrm{E} 8$ & 0.07 & $E, F$ & II \\
\hline $\mathrm{NO}_{3}$ & $"$ & $"$ & No & Yes & $\mathrm{NO}_{3}^{-}$ & 32.9 & $3.29 E 7$ & 10 & $F$ & III \\
\hline Nickel & $"$ & $"$ & No & Yes & $\mathrm{Ni}^{++}$ & .009 & $1.80 \mathrm{E} 5$ & 0.05 & $E, F$ & 11 \\
\hline Chromium & $"$ & $"$ & No & Yes & $\mathrm{Cr}^{++}\left(\mathrm{Cr}_{2} \mathrm{O}_{7}\right)$ & .009 & $4.50 \mathrm{E} 5$ & 0.02 & $E, F$ & II \\
\hline Copper & $"$ & $"$ & No & Yes & $\mathrm{Cu}^{++}$ & .011 & $1.10 \mathrm{E} 5$ & .1 & $r$ & I I I \\
\hline Phosphorus & $"$ & $"$ & No & Yes & $\mathrm{PO}_{4}^{---}$ & .091 & $9.10 \mathrm{E} 6$ & .1 & c & II \\
\hline Chlorine & $"$ & $"$ & No & Yes & $\mathrm{Cl}^{-}$ & 1.30 & $4.33 \quad E 10$ & .15 & $\mathrm{~J}$ & I I \\
\hline $\mathrm{SO}_{4}$ & $"$ & $"$ & No & Yes & $\mathrm{SO}_{4}$ & 9.58 & $9.16 \mathrm{E} 4$ & 50 & $E, F$ & I I \\
\hline BOD & & $\begin{array}{l}\text { Treated sanitary } \\
\text { wastes }\end{array}$ & No & Yes & & 2.28 & & & & \\
\hline $\mathrm{CaO}$ & & & No & & $\mathrm{Ca} 0$ & negligible & & 30 & $E, F$ & II \\
\hline $\mathrm{CaF}_{2}$ & & & No & & $\mathrm{CaF}_{2}$ & negligible & & & $E, F$ & I I \\
\hline
\end{tabular}

(a) Based on release numbers from Reference 1, Appendix C (NED0-20197, Environmental Report, General Electric Nuclear Facility, Wilmington, N.C., General Electric Co., January 1974), Table 4-3 and a plant capacity of 725.4 MTU/year deduced from

Reference 1 , Appendix $C$, note at the bottom of Table i-l. 
1 


\section{DISTRIBUTION}

No. of

Copies

OFFSITE

A. A. Churm

DOE Chicago Patent Group

Chicago Operations Office

9800 South Cass Avenue

Argonne, IL 60439

R. W. Ramsey

DOE Division of Environmental Control Engineering

Washington, DC 20545

W. E. Mott

DOE Division of Environmental Control Engineering

Washington, DC 20545

J. Counts

DOE Division of Environmental Engineering

Washington, DC 20545

T. J. Kabele

The Analytical Sciences

Corporation

6 Jacob Way

Reading, MA 01867

27 Technical Information Service

\section{ONSITE}

DOE Richland Operations

H. E. Ransom

66 Pacific Northwest Laboratory
R. L. Aaberg
M. H. Arndt
W. J. Bailey
W. J. Bair

No. of

Copies

D. B. Cearlock

L. L. Clark

C. Cowan

G. W. Dawson

W. I. Ender 1 in

R. M. Fleischman

J. C. Fox

A. J. Haverfield

C. M. Heeb (10)

D. L. Hesse 1

J. H. Jarrett

W. E. Kennedy, Jr.

H. V. Larson

D. H. Lester

M. A. Lewallen (10)

R. C. Liikala

R. W. McKee

D. E. Newman

J. M. Nielsen

H. D. Oak

E. L. Owzarski

A. M. Platt

T. B. Powers

C. L. Simpson

B. E. Vaughn

E. C. Watson

W. R. Wiley

L. D. Williams

J. K. Young

Technical Information (5)

Publishing Coordination WI 
